\title{
Highly site-selective formation of perfluoroalkylated anilids via a protecting strategy by molybdenum hexacarbonyl catalyst
}

Chunchen Yuan, Ping Dai, Xiaoguang Bao* and Yingsheng Zhao*

\section{Supporting information}

Table of Contents

\begin{tabular}{|c|l|l|}
\hline 1. & Reagents & S2 \\
\hline 2. & Instruments & S2 \\
\hline 3. & Computational Method & S2 \\
\hline 4. & Table 1s: Details for optimization & S2 \\
\hline 5. & General procedures for para-perfluoroalkylation of anilides & S5 \\
\hline 6. & Transformations and applications & S13 \\
\hline 7. & Transformations & S17 \\
\hline 8. & NOESY analysis of 4a & S18 \\
\hline 9. & Preliminary mechanism studies & S20 \\
\hline 10. & Cartesian Coordinates and Energies & S21 \\
\hline & References & S29 \\
\hline & NMR spectra & S30 \\
\hline
\end{tabular}


1. Reagents: Unless otherwise noted, all reagents purchased from commercial suppliers and used without further purification. Column chromatography purifications performed using 200-300 mesh silica gel.

2. Instruments: NMR spectra recorded on Varian Inova- $400 \mathrm{MHz}$, Inova-300 $\mathrm{MHz}$, Bruker DRX-400 or Bruker DRX-500 instruments and calibrated using residual solvent peaks as internal reference. Multiplicities are recorded as: $\mathrm{s}=$ singlet, $\mathrm{d}=$ doublet, $\mathrm{t}=$ triplet, $\mathrm{q}=$ quartet, $\mathrm{dd}=$ doublet of doublets, $\mathrm{dt}=$ doublet of triplets, brs $=$ broad singlet, $\mathrm{m}=$ multiplet. HRMS analysis carried out using a Bruker micrOTOF-Q instrument or a TOF-MS instrument.

\section{Computational Method}

All the computations were carried out using the B3LYP density functional method. ${ }^{1}$ The SDD basis set in conjunction with the SDD pseudopotential ${ }^{2}$ was used for the Mo atom. The 6-31G(d) basis $\operatorname{set}^{3}$ was used for other atoms in the geometry optimizations. Vibrational frequency was calculated to characterize stationary points as local minima or transition states which were checked by the calculations of intrinsic reaction coordinate (IRC) ${ }^{4}$. To consider the solvation effect in dimethylsulfoxide, the single-point energy computations were performed using the SMD model ${ }^{5}$ at the optimized gas-phase geometries. On the basis of the optimized geometries, the B3LYP functional with larger basis set (SDD for Mo and 6-311++G(d,p) for other atoms) were utilized for single-point energy calculations on stationary points. The Gibbs free energy of solvation was determined by adding the solvation single-point energy and the gas-phase thermal correction to the Gibbs free energy obtained from the vibrational frequencies. All calculations were performed with the Gaussian 09 suite of programs ${ }^{6}$. The 3D structures of the studied species were shown using the CYLView software. ${ }^{7}$

\section{Table 1s: Details for optimization ${ }^{\mathrm{a}}$}

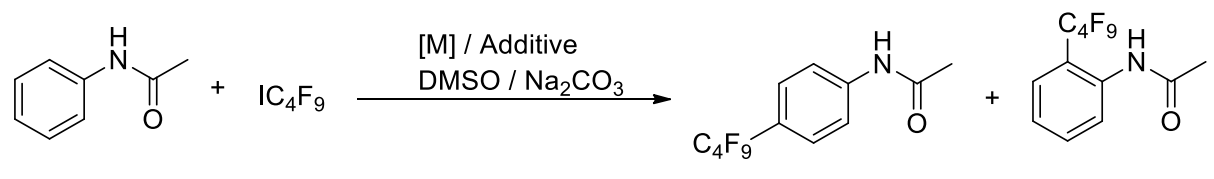

$1 \mathrm{a}$

$2 \mathbf{3}$

$3 a^{\prime}$

\begin{tabular}{cccccc}
\hline Entry & Catalyst $(\mathrm{M} 10$ mol\%) & Ligand & Additive & $\begin{array}{c}\mathbf{3 a}(\%, \\
\mathbf{G C})\end{array}$ & $\begin{array}{c}\mathbf{3 a} \boldsymbol{\mathbf { 9 }}(\mathbf{\%}) \\
\mathbf{G C})\end{array}$ \\
\hline 1 & {$\left[\mathrm{RuCl}_{2}(p-\mathrm{cymene})\right]_{2}$} & - & $\mathrm{AgNFf}_{2}(20 \mathrm{~mol} \%)$ & 15 & - \\
2 & $\mathrm{Ru}_{3}(\mathrm{CO})_{12}$ & - & - & 19 & trace \\
3 & $\mathrm{Cr}(\mathrm{CO})_{6}$ & - & - & 18 & trace \\
4 & $\mathrm{Fe}(\mathrm{CO})_{9}$ & - & - & trace & trace \\
5 & $\mathrm{Mo}(\mathrm{CO})_{6}$ & - & - & 26 & trace \\
6 & $\mathrm{Mo}(\mathrm{CO})_{6}$ & - & $\mathrm{CO}$ & 43 & trace \\
7 & $\mathrm{Mo}(\mathrm{CO})_{6}$ & $N-\mathrm{Ac}-\mathrm{Gly-OH}$ & $\mathrm{CO}$ & 33 & trace
\end{tabular}




\begin{tabular}{cccccc}
8 & $\mathrm{Mo}(\mathrm{CO})_{6}$ & $\mathrm{PivOH}$ & $\mathrm{CO}$ & 37 & trace \\
9 & $\mathrm{Mo}(\mathrm{CO})_{6}$ & $\mathrm{PPh}_{3}$ & $\mathrm{CO}$ & 28 & trace \\
10 & $\mathrm{Mo}(\mathrm{CO})_{6}$ & - & $\mathrm{CO} / \mathrm{KF}^{\mathrm{b}}$ & 68 & trace \\
11 & $\mathrm{Mo}(\mathrm{CO})_{6}$ & & $\mathrm{CO} / \mathrm{KF}^{\mathrm{bc}}$ & $86\left(80^{\mathrm{d}}\right)$ & trace \\
12 & & & $\mathrm{CO} / \mathrm{KF}^{\mathrm{b}}$ & None & None \\
\hline
\end{tabular}

${ }^{\text {a }}$ Conditions: Reaction performed at a $0.2 \mathrm{mmol}$ scale with 2a (3 equiv) in a sealed tube, DMSO (1 $\mathrm{mL}), \mathrm{Na}_{2} \mathrm{CO}_{3}$ (2 equiv), $120{ }^{\circ} \mathrm{C}, 24 \mathrm{~h} ;{ }^{\mathrm{b}} 140^{\circ} \mathrm{C}, \mathrm{KF}$ (2 equiv). ${ }^{\mathrm{c}} 48 \mathrm{~h} .{ }^{\mathrm{d}}$ Isolated yield.

\section{More details}

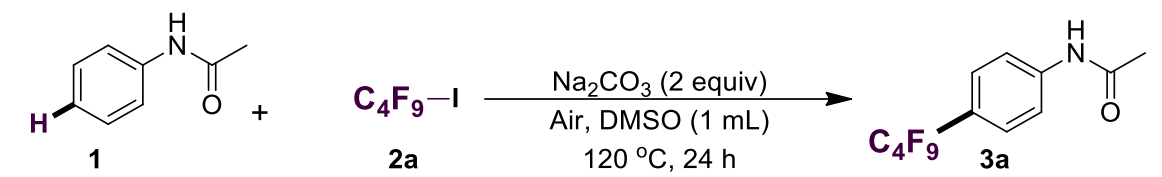

\begin{tabular}{|c|c|c|c|}
\hline Catalyst (10 mol \%) & Yield $(\%$, GC) & Catalyst (10 mol \%) & Yield $(\%$, GC) \\
\hline $\mathrm{Mn}(\mathrm{OAc})_{2}$ & No reaction & $\mathrm{Fe}(\mathrm{acac})_{2}$ & 7 \\
\hline $\mathrm{Ni}\left(\mathrm{PPh}_{3}\right)_{2} \mathrm{Cl}_{2}$ & No reaction & $\mathrm{Fe}(\mathrm{CO})_{9}$ & trace \\
\hline $\mathrm{CuBr}$ & No reaction & $\mathrm{Ni}(\text { acac })_{2}$ & 12 \\
\hline$[\mathrm{Rh}(\mathrm{COD}) \mathrm{Cl}]_{2}$ & 4 & $\mathrm{FeI}(\mathrm{CO})_{2}$ Cyclopentane & trace \\
\hline $\mathrm{Mo}(\mathrm{CO})_{6}$ & 26 & $\mathrm{Co}(\mathrm{acac})_{2} \cdot \mathrm{H}_{2} \mathrm{O}$ & trace \\
\hline $\mathrm{AlCl}_{3}$ & 10 & {$\left[\mathrm{Ru}(p \text {-Cymene }) \mathrm{Cl}_{2}\right]_{2}$} & 15 \\
\hline $\mathrm{Ni}($ dppe $) \mathrm{Cl}_{2}$ & trace & $\mathrm{Cr}(\mathrm{CO})_{6}$ & 18 \\
\hline $\mathrm{Ni}(\mathrm{dppp}) \mathrm{Cl}_{2}$ & trace & $\mathrm{Ru}_{3}(\mathrm{CO})_{12}$ & 19 \\
\hline
\end{tabular}

${ }^{\text {a }}$ Conditions: Reaction performed at a $0.2 \mathrm{mmol}$ scale with 2a (3 equiv) in a sealed tube, DMSO (1 $\mathrm{mL}$ ), $\mathrm{Na}_{2} \mathrm{CO}_{3}$ (2 equiv), $120^{\circ} \mathrm{C}, 24 \mathrm{~h}$;

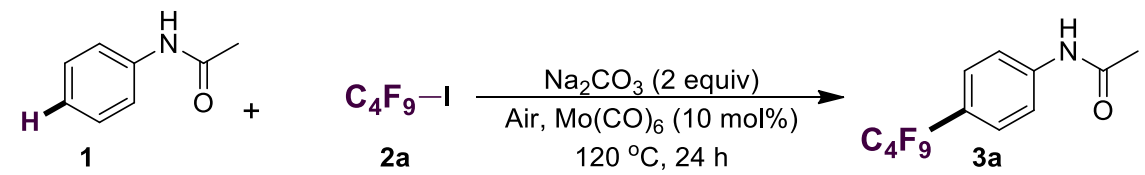

\begin{tabular}{|l|c|l|c|}
\hline Solvent & Yield $(\boldsymbol{\%}, \mathbf{G C})$ & Solvent & Yield $(\boldsymbol{\%}, \mathbf{G C})$ \\
\hline toluene & No reaction & dioxane & trace \\
\hline DCE & trace & DME & trace \\
\hline DMF & 19 & BnCN & No reaction \\
\hline HFIP & trace & MeCN & No reaction \\
\hline DMSO & 26 & $n$-Hexane & 20 \\
\hline
\end{tabular}

${ }^{\text {a }}$ Conditions: Reaction performed at a 0.2 mmol scale with 2a (3 equiv) in a sealed tube, solvent ( 1 $\mathrm{mL}$ ), $\mathrm{Na}_{2} \mathrm{CO}_{3}$ (2 equiv), $120{ }^{\circ} \mathrm{C}, 24 \mathrm{~h}$;<smiles>CC(=O)Nc1ccccc1</smiles>

1<smiles>CC(=O)Nc1ccc(C(F)(F)F)cc1</smiles>

\begin{tabular}{|c|c|c|c|}
\hline $\begin{array}{c}\text { Atmosphere (1 } \\
\text { atm) }\end{array}$ & Yield (\%, GC) & Atmosphere (1 atm) & Yield (\%, GC) \\
\hline
\end{tabular}




\begin{tabular}{|l|c|l|c|}
\hline air & 26 & $\mathrm{~N}_{2}$ & 27 \\
\hline $\mathrm{CO}$ & 43 & $\mathrm{Ar}$ & 25 \\
\hline $\mathrm{O}_{2}$ & 6 & & \\
\hline
\end{tabular}

${ }^{\text {a }}$ Conditions: Reaction performed at a $0.2 \mathrm{mmol}$ scale with $\mathbf{2 a}$ (3 equiv) in a sealed tube, $\mathrm{Mo}(\mathrm{CO})_{6}$ (10 mol\%), DMSO (1 mL), $\mathrm{Na}_{2} \mathrm{CO}_{3}$ (2 equiv), $120{ }^{\circ} \mathrm{C}, 24 \mathrm{~h}$;

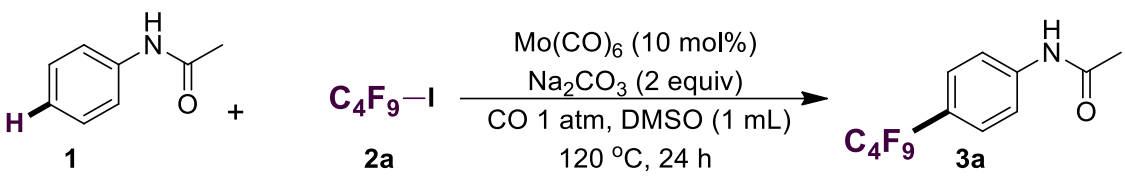

\begin{tabular}{|l|c|l|c|}
\hline $\begin{array}{c}\text { Additive (30 } \\
\text { mol\%) }\end{array}$ & Yield (\%, GC) & Additive (30 mol\%) & Yield (\%, GC) \\
\hline PivOH & 37 & $N-A c-G l y-O H$ & 33 \\
\hline 1-AdOH & 35 & NaF & 47 \\
\hline $\mathrm{MesCOOH}$ & 42 & $\mathrm{KF}$ & 60 \\
\hline $\mathrm{PPh}_{3}$ & 28 & $\mathrm{CsF}$ & 52 \\
\hline $\mathrm{dppb}$ & 25 & $\mathrm{KF}(2$ equiv) & 68 \\
\hline
\end{tabular}

${ }^{a}$ Conditions: Reaction performed at a $0.2 \mathrm{mmol}$ scale with $2 \mathbf{a}$ (3 equiv) in a sealed tube, $\mathrm{Mo}(\mathrm{CO})_{6}$ (10 mol\%), DMSO (1 mL), $\mathrm{Na}_{2} \mathrm{CO}_{3}$ (2 equiv), $\mathrm{CO}(1 \mathrm{~atm}), 120^{\circ} \mathrm{C}, 24 \mathrm{~h}$;<smiles>CC(=O)Nc1ccccc1</smiles>

1<smiles>[AsH2+]I</smiles>

2a

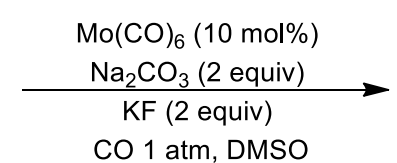

$120^{\circ} \mathrm{C}, 24 \mathrm{~h}$

\begin{tabular}{|l|c|l|c|}
\hline \multicolumn{1}{|c|}{ DMSO } & Yield (\%, GC) & DMSO & Yield (\%, GC) \\
\hline $0.5 \mathrm{ml}$ & 52 & $2.0 \mathrm{ml}$ & 54 \\
\hline $1 \mathrm{ml}$ & 68 & $2.5 \mathrm{ml}$ & 50 \\
\hline $1.5 \mathrm{ml}$ & 53 & & \\
\hline
\end{tabular}

${ }^{a}$ Conditions: Reaction performed at a $0.2 \mathrm{mmol}$ scale with $\mathbf{2 a}$ (3 equiv) in a sealed tube, $\mathrm{Mo}(\mathrm{CO})_{6}$ (10 mol\%), DMSO, $\mathrm{Na}_{2} \mathrm{CO}_{3}$ (2 equiv), $\mathrm{KF}$ (2 equiv), $\mathrm{CO}(1 \mathrm{~atm}), 120^{\circ} \mathrm{C}, 24 \mathrm{~h}$;<smiles>CC(=O)Nc1ccccc1</smiles>

1

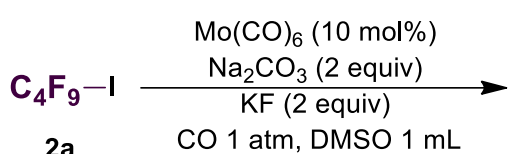

$T, 24 \mathrm{~h}$

\begin{tabular}{|l|c|l|c|}
\hline \multicolumn{1}{|c|}{ Temperature } & Yield $(\boldsymbol{\%}, \mathbf{G C})$ & Temperature & Yield (\%, GC) \\
\hline r.t. & No reaction & $100{ }^{\circ} \mathrm{C}$ & 16 \\
\hline $50{ }^{\circ} \mathrm{C}$ & No reaction & $120^{\circ} \mathrm{C}$ & 68 \\
\hline $80{ }^{\circ} \mathrm{C}$ & trace & $140{ }^{\circ} \mathrm{C}$ & 74 \\
\hline $140{ }^{\circ} \mathrm{C}(48 \mathrm{~h})$ & $86\left(80^{\mathrm{b}}\right)$ & & \\
\hline
\end{tabular}

${ }^{a}$ Conditions: Reaction performed at a $0.2 \mathrm{mmol}$ scale with $2 \mathrm{a}$ ( 3 equiv) in a sealed tube, $\mathrm{Mo}(\mathrm{CO})_{6}$ (10 mol\%), DMSO (1 mL), $\mathrm{Na}_{2} \mathrm{CO}_{3}$ (2 equiv), KF (2 equiv), $\mathrm{CO}(1 \mathrm{~atm}), 120{ }^{\circ} \mathrm{C}, 24 \mathrm{~h}$; ${ }^{b}$ isolated yelid.

Note: We performed acetanilide under the condition according the work by Zhou and Huang (J. Fluorine Chem. 1988, 39, 87). However, only starting materials recovered. 


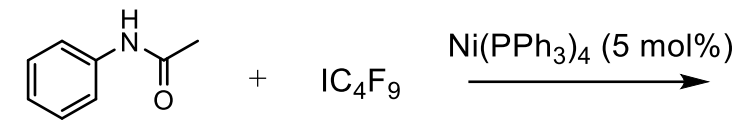

2 equiv

\begin{tabular}{|c|c|c|}
\hline Solvent & Temperature $\left({ }^{\circ} \mathrm{C}\right)$ & Result \\
\hline Dioxane & 80 & No reaction \\
\hline Dioxane & 80 & No reaction \\
\hline Dioxane & 140 & No reaction \\
\hline DMSO & 80 & No reaction \\
\hline DMSO & 100 & No reaction \\
\hline DMSO & 140 & No reaction \\
\hline
\end{tabular}

\section{General procedures for para-perfluoroalkylation of anilides}<smiles>[R]Nc1cccc(C)c1</smiles>

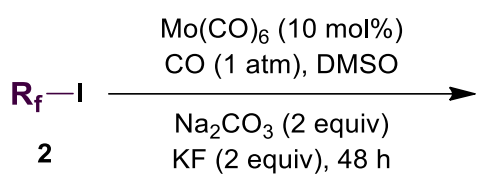<smiles>[R]Nc1ccc([R])c(S([CH])(=O)=O)c1</smiles>

General Procedures: A mixture of 1 ( $0.2 \mathrm{mmol}, 1.0$ equiv), 2 (3 equiv), $\mathrm{Mo}(\mathrm{CO})_{6}(5.3 \mathrm{mg}, 10$ mmol \%), $\mathrm{Na}_{2} \mathrm{CO}_{3}$ (41.0 mg, 2 equiv), KF (23.2 mg, $30 \mathrm{mmol} \%$ ) and DMSO (1 mL) in a $15 \mathrm{~mL}$ glass vial sealed under $\mathrm{CO}$ atmosphere was heated at $140{ }^{\circ} \mathrm{C}$ for 48 hours. The reaction mixture cooled to room temperature and concentrated in vacuo. The resulting residue was purified by column chromatography (PE / EA = 5:1) on silica gel to give the product 3 or 4 .

Note: For the ${ }^{13} \mathrm{C}$ NMR (F coupled), peaks for the $\mathrm{C}$ of perfluoroalkyl chain and $\mathrm{C}$ adjacent to perfluoroalkyl chain (in most cases) are too broad to be assigned (not shown in the following data). In addition, we used trifluoromethyl iodide in DMF solution purchased from commercial suppliers (trifluoromethyl iodide in DMF, $\omega=25 \%$ ).<smiles>CC(=O)Nc1ccc(C(F)(F)C(F)(F)C(F)(F)C(F)(F)F)cc1</smiles>

White solid, 56.3mg, 80\%. (PE / EA = $5: 1)$

${ }^{1} \mathbf{H}$ NMR (400 MHz, DMSO) $\delta 10.32(\mathrm{~s}, 1 \mathrm{H}), 7.81(\mathrm{~d}, J=8.7 \mathrm{~Hz}, 2 \mathrm{H}), 7.59$ (d, $\left.J=8.6 \mathrm{~Hz}, 2 \mathrm{H}\right)$, $2.09(\mathrm{~s}, 3 \mathrm{H})$.

${ }^{19}$ F NMR (376 MHz, DMSO) $\delta-78.38--83.00(3 \mathrm{~F}),-108.06--110.37$ (2F), -121.50 - -123.56 (2F), $-124.27--126.54(2 \mathrm{~F})$.

${ }^{13}$ C NMR (101 MHz, DMSO) $\delta 169.05,143.15,127.49\left(\mathrm{t}, J_{C-F}=6.3 \mathrm{~Hz}\right), 120.83\left(\mathrm{t}, J_{C-F}=24.4\right.$ $\mathrm{Hz}), 118.78,24.11$.

HRMS Calcd for $\mathrm{C}_{12} \mathrm{H}_{8} \mathrm{~F}_{9} \mathrm{NO}\left[\mathrm{M}+\mathrm{H}^{+}\right]$: 354.0540; Found: 354.0534 . 
$\overbrace{\mathrm{H}}$

White solid, 27.1mg, 50\%. (PE / EA = 5:1)

${ }^{1} \mathbf{H}$ NMR $\left(400 \mathrm{MHz}, \mathrm{CDCl}_{3}\right) \delta 7.65(\mathrm{~d}, J=8.5 \mathrm{~Hz}, 2 \mathrm{H}), 7.54(\mathrm{~d}, J=8.7 \mathrm{~Hz}, 2 \mathrm{H}), 7.40(\mathrm{~s}, 1 \mathrm{H}), 2.21$ (s, 3H).

${ }^{19}$ F NMR $\left(376 \mathrm{MHz}, \mathrm{CDCl}_{3}\right) \delta-84.61--85.12(3 \mathrm{~F}),-114.47(2 \mathrm{~F})$.

${ }^{13}$ C NMR $\left(101 \mathrm{MHz}, \mathrm{CDCl}_{3}\right) \delta 168.69,141.22,127.62\left(\mathrm{t}, J_{C-F}=6.3 \mathrm{~Hz}\right), 119.46,24.89$.

HRMS Calcd for $\mathrm{C}_{10} \mathrm{H}_{8} \mathrm{~F}_{5} \mathrm{NO}\left[\mathrm{M}+\mathrm{Na}^{+}\right]$: 276.0424; Found: 276.0409 .<smiles>CC(=O)Nc1ccc(C(F)(F)C(F)(F)C(F)(F)F)cc1</smiles>

White solid, 33.1mg, 55\%. (PE / EA = 5:1)

${ }^{1}$ H NMR (400 MHz, DMSO) $\delta 10.34(\mathrm{br}, 1 \mathrm{H}), 7.80(\mathrm{~d}, J=8.7 \mathrm{~Hz}, 2 \mathrm{H}), 7.59(\mathrm{~d}, J=8.7 \mathrm{~Hz}, 2 \mathrm{H})$, $2.08(\mathrm{~s}, 3 \mathrm{H})$.

${ }^{19}$ F NMR (376 MHz, DMSO) $\delta$-79.53 (3F), -109.96 (2F), -126.20 (2F).

${ }^{13}$ C NMR (101 MHz, DMSO) $\delta 169.24,143.17,128.75,127.50\left(\mathrm{t}, J_{C-F}=6.5 \mathrm{~Hz}\right), 118.94,24.19$.

HRMS Calcd for $\mathrm{C}_{11} \mathrm{H}_{8} \mathrm{~F}_{7} \mathrm{NO}\left[\mathrm{M}+\mathrm{H}^{+}\right]$: 304.0572; Found: 304.0582<smiles>CC(=O)Nc1ccc(C(F)(F)C(F)(F)C(F)(F)C(F)(F)C(F)(F)F)cc1</smiles>

White solid, 62.0mg, 77\%. (PE / EA = $5: 1$ )

${ }^{1}$ H NMR (400 MHz, DMSO) $\delta 10.32(\mathrm{br}, 1 \mathrm{H}), 7.81(\mathrm{~d}, J=8.7 \mathrm{~Hz}, 2 \mathrm{H}), 7.60(\mathrm{~d}, J=8.7 \mathrm{~Hz}, 2 \mathrm{H})$, $2.09(\mathrm{~s}, 3 \mathrm{H})$.

${ }^{19}$ F NMR (376 MHz, DMSO) $\delta-80.43$ (3F), -107.77 - -110.03 (2F), -118.59 - -124.37 (4F), $-126.01(2 \mathrm{~F})$.

${ }^{13}$ C NMR (101 MHz, DMSO) $\delta 169.03,143.14,127.50\left(\mathrm{t}, J_{C-F}=6.2 \mathrm{~Hz}\right), 120.85\left(\mathrm{t}, J_{C-F}=24.2\right.$ $\mathrm{Hz}), 118.76,24.11$.

HRMS Calcd for $\mathrm{C}_{13} \mathrm{H}_{8} \mathrm{~F}_{11} \mathrm{NO}\left[\mathrm{M}+\mathrm{H}^{+}\right]$: 404.0508; Found: 404.0505 .<smiles>CC(=O)Nc1ccc(C(F)(F)C(F)(F)C(F)(F)C(F)(F)C(F)(F)C(F)(F)F)cc1</smiles>

White solid, 72.2mg, 76\%. (PE / EA = 5:1)

${ }^{1}$ H NMR (400 MHz, DMSO) $\delta 10.32(\mathrm{br}, 1 \mathrm{H}), 7.81(\mathrm{~d}, J=8.7 \mathrm{~Hz}, 2 \mathrm{H}), 7.58(\mathrm{dd}, J=8.6,4.3 \mathrm{~Hz}$, $2 \mathrm{H}), 2.08(\mathrm{~s}, 3 \mathrm{H})$.

${ }^{19}$ F NMR (376 MHz, DMSO) $\delta$-80.60 (3F), -109.18 (2F), -121.66 (4F), -122.90 (2F), -126.09 (2F).

${ }^{13}$ C NMR (101 MHz, DMSO) $\delta 169.01,143.14,127.41,120.90\left(\mathrm{t}, J_{C-F}=25.0 \mathrm{~Hz}\right), 118.71,24.08$. 
HRMS Calcd for $\mathrm{C}_{14} \mathrm{H}_{8} \mathrm{~F}_{13} \mathrm{NO}$ [M+Na+ $]$ : 476.0296; Found: 476.0313.<smiles>CC(=O)Nc1ccc(C(F)(F)C(F)(F)C(F)(F)C(F)(F)C(F)(F)C(F)(F)C(F)(F)C(F)(F)F)cc1</smiles>

White solid, $81.5 \mathrm{mg}, 81 \%$. (PE / EA = 5:1)

${ }^{1}$ H NMR (400 MHz, DMSO) $\delta 10.32(\mathrm{br}, 1 \mathrm{H}), 7.81(\mathrm{~d}, J=8.6 \mathrm{~Hz}, 2 \mathrm{H}), 7.57(\mathrm{~d}, J=8.7 \mathrm{~Hz}, 2 \mathrm{H})$, $2.08(\mathrm{~s}, 3 \mathrm{H})$.

${ }^{19}$ F NMR (376 MHz, DMSO) $\delta$-80.45 (3F), -108.03 - -109.74 (2F), -121.91 (8F), -125.94 (2F).

${ }^{13}$ C NMR (101 MHz, DMSO) $\delta 169.04,143.14,127.48\left(\mathrm{t}, J_{C-F}=6.4 \mathrm{~Hz}\right), 118.75,24.11$.

HRMS Calcd for $\mathrm{C}_{15} \mathrm{H}_{8} \mathrm{~F}_{15} \mathrm{NO}\left[\mathrm{M}+\mathrm{H}^{+}\right]$: 504.0445; Found: 504.0433 .

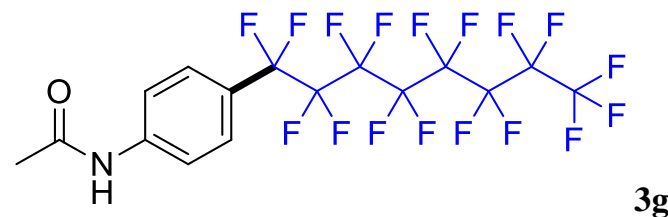

White solid, 100.0mg, 87\%. (PE / EA = $5: 1$ )

${ }^{1}$ H NMR (400 MHz, DMSO) $\delta 10.30(\mathrm{br}, 1 \mathrm{H}), 7.79(\mathrm{~d}, J=8.7 \mathrm{~Hz}, 2 \mathrm{H}), 7.54(\mathrm{~d}, J=8.8 \mathrm{~Hz}, 2 \mathrm{H})$, $2.07(\mathrm{~s}, 3 \mathrm{H})$.

${ }^{19}$ F NMR (376 MHz, DMSO) $\delta-80.96(3 \mathrm{~F}),-108.53--110.11(2 \mathrm{~F}),-117.57--125.80(10 \mathrm{~F})$, $-125.86--127.75(2 \mathrm{~F})$.

${ }^{13}$ C NMR (101 MHz, DMSO) $\delta 168.98,143.08,128.63,127.34\left(\mathrm{t}, J_{C-F}=5.9 \mathrm{~Hz}\right), 118.65,24.08$.

HRMS Calcd for $\mathrm{C}_{16} \mathrm{H}_{8} \mathrm{~F}_{17} \mathrm{NO}$ [M+Na+ $]$ : 576.0232; Found: 576.0241.

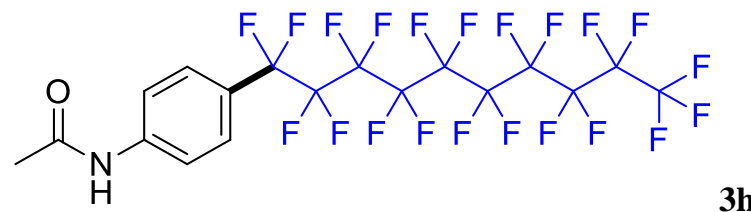

White solid, 75.4mg, 56\%. (PE / EA = 5: 1)

${ }^{1}$ H NMR (400 MHz, DMSO) $\delta 10.25(\mathrm{br}, 1 \mathrm{H}), 7.70(\mathrm{~d}, J=8.7 \mathrm{~Hz}, 2 \mathrm{H}), 7.39(\mathrm{~d}, J=8.8 \mathrm{~Hz}, 2 \mathrm{H})$, $2.05(\mathrm{~s}, 3 \mathrm{H})$.

${ }^{19}$ F NMR (376 MHz, DMSO) $\delta-81.96$ (3F), $-109.75--111.33(2 \mathrm{~F}),-122.85$ (15F), $-127.16(2 \mathrm{~F})$.

${ }^{13}$ C NMR (101 MHz, DMSO) $\delta 168.89,143.23,126.93,121.21\left(\mathrm{t}, J_{C-F}=23.5 \mathrm{~Hz}\right), 118.10,23.62$.

HRMS Calcd for $\mathrm{C}_{18} \mathrm{H}_{8} \mathrm{~F}_{21} \mathrm{NO}$ [M+Na+ $]$ : 676.0168; Found: 676.0170.<smiles>CC(=O)Nc1ccc(C(F)(C(F)(F)F)C(F)(F)F)cc1</smiles>

White solid, 13.5mg, 21\%. (PE / EA = 5: 1)

${ }^{1}$ H NMR (400 MHz, DMSO) $\delta 10.30(\mathrm{br}, 1 \mathrm{H}), 7.81(\mathrm{~d}, J=8.7 \mathrm{~Hz}, 2 \mathrm{H}), 7.59(\mathrm{~d}, J=8.6 \mathrm{~Hz}, 2 \mathrm{H})$, $2.08(\mathrm{~s}, 3 \mathrm{H})$.

${ }^{19}$ F NMR (376 MHz, DMSO) $\delta-75.27$ (6F), $-181.30(1 \mathrm{~F})$.

${ }^{13}$ C NMR (101 MHz, DMSO) $\delta 169.02,142.22,128.68\left(\mathrm{~d}, J_{C-F}=1.5 \mathrm{~Hz}\right), 126.21\left(\mathrm{~d}, J_{C-F}=10.9\right.$ $\mathrm{Hz}), 119.26,24.10$. 
HRMS Calcd for $\mathrm{C}_{11} \mathrm{H}_{8} \mathrm{~F}_{7} \mathrm{NO}\left[\mathrm{M}+\mathrm{Na}^{+}\right]$: 326.0392; Found: 326.0388 .<smiles>CC(=O)Nc1ccc(C(F)(F)F)cc1</smiles>

White solid, 6.5mg, 16\%. (PE / EA = 5:1)

${ }^{1} \mathrm{H}$ NMR $\left(400 \mathrm{MHz}, \mathrm{CDCl}_{3}\right) \delta 7.76(\mathrm{~s}, 1 \mathrm{H}), 7.64(\mathrm{~d}, J=7.4 \mathrm{~Hz}, 2 \mathrm{H}), 7.55(\mathrm{~d}, J=7.8 \mathrm{~Hz}, 2 \mathrm{H}), 2.20$ (s, 3H).

${ }^{13}$ C NMR $\left(101 \mathrm{MHz}, \mathrm{CDCl}_{3}\right) \delta 168.90,141.09,126.38\left(\mathrm{q}, J_{C-F}=4.0 \mathrm{~Hz}\right), 125.92,124.19\left(\mathrm{q}, J_{C-F}=\right.$ $259.67 \mathrm{~Hz}), 119.51,24.78$.

${ }^{13}$ C NMR (101 MHz, DMSO) $\delta-62.15$.

This in a know compound.<smiles>COc1cc(NC(C)=O)ccc1C(F)(F)C(F)(F)C(F)(F)C(F)(F)C(F)(F)C(F)(F)C(F)(F)C(F)(F)F</smiles>

White solid, 101.2mg, 87\%. (PE / EA = 5:1)

${ }^{1} \mathrm{H}$ NMR (400 MHz, DMSO) $\delta 10.24(\mathrm{br}, 1 \mathrm{H}), 7.54(\mathrm{~s}, 1 \mathrm{H}), 7.32(\mathrm{~d}, J=8.7 \mathrm{~Hz}, 1 \mathrm{H}), 7.24(\mathrm{~d}, J=$ $8.8 \mathrm{~Hz}, 1 \mathrm{H}), 3.71(\mathrm{~s}, 3 \mathrm{H}), 2.06$ (s, 3H).

${ }^{19}$ F NMR (376 MHz, DMSO) $\delta-77.37--85.05$ (3F), $-105.12--107.81$ (2F), $-114.94--125.98$ (10F), $-126.25--129.48(2 \mathrm{~F})$.

${ }^{13}$ C NMR (101 MHz, DMSO) $\delta 169.04,158.52,144.69,129.43,129.05\left(\mathrm{t}, J_{C-F}=8.8 \mathrm{~Hz}\right), 110.23$, 102.71, 55.51, 24.12.

HRMS Calcd for $\mathrm{C}_{17} \mathrm{H}_{10} \mathrm{~F}_{17} \mathrm{NO}_{2}\left[\mathrm{M}+\mathrm{H}^{+}\right]$: 584.0518; Found: 584.0500 .

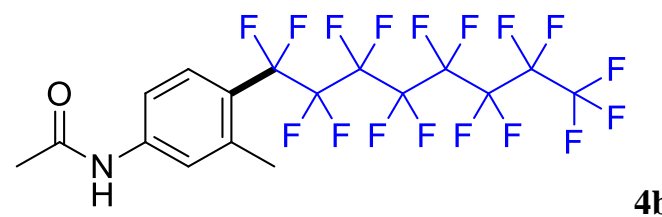

White solid, 79.2mg, 70\%. (PE / EA = 5: 1)

${ }^{1}$ H NMR (400 MHz, DMSO) $\delta 10.15(\mathrm{br}, 1 \mathrm{H}), 7.50(\mathrm{~d}, J=11.4 \mathrm{~Hz}, 2 \mathrm{H}), 7.27(\mathrm{~d}, J=8.5 \mathrm{~Hz}, 1 \mathrm{H})$, $2.32(\mathrm{~s}, 3 \mathrm{H}), 2.04$ (s, 3H).

${ }^{19}$ F NMR (376 MHz, DMSO) $\delta$-81.99 (3F), -105.93 (2F), -122.63 (10F), -127.07 (2F).

${ }^{13}$ C NMR (101 MHz, DMSO) $\delta 168.83,142.95,138.25$ (t, $\left.J_{C-F}=3.0 \mathrm{~Hz}\right), 137.62,128.60,121.26$, $115.54,23.49,19.32$.

HRMS Calcd for $\mathrm{C}_{17} \mathrm{H}_{10} \mathrm{~F}_{17} \mathrm{NO}\left[\mathrm{M}+\mathrm{Na}^{+}\right]$: 590.0383 ; Found: 590.0361 .

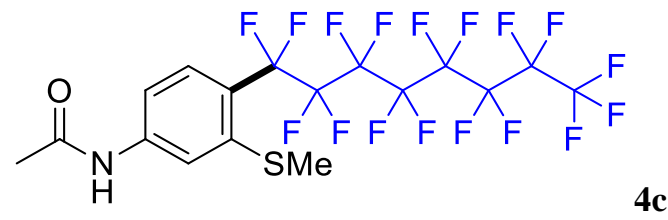

White solid, 75.1mg, 63\%. (PE / EA = $5: 1$ )

${ }^{1} \mathrm{H}$ NMR (400 MHz, DMSO) $\delta 9.28(\mathrm{br}, 1 \mathrm{H}), 7.44(\mathrm{~d}, J=8.5 \mathrm{~Hz}, 1 \mathrm{H}), 7.37$ (s, 1H), $7.27-7.19(\mathrm{~m}$, $1 \mathrm{H}), 2.54$ (s, 3H), 2.02 (s, 3H)

${ }^{19}$ F NMR (376 MHz, DMSO) $\delta$-82.28 (3F), -105.76 (2F), -120.84 - -124.18 (10F), -127.24 (2F). 
${ }^{13}$ C NMR (101 MHz, DMSO) $\delta 168.93,145.39,137.75\left(\mathrm{t}, J_{C-F}=6.0 \mathrm{~Hz}\right), 128.11,125.88,122.17$, $120.21,22.35,13.58$.

HRMS Calcd for $\mathrm{C}_{17} \mathrm{H}_{10} \mathrm{~F}_{17} \mathrm{NOS}\left[\mathrm{M}+\mathrm{Na}^{+}\right]$: 622.0109; Found: 622.0118 .<smiles>CC(=O)Nc1ccc(C(F)(F)C(F)(F)C(F)(F)C(F)(F)C(F)(F)C(F)(F)C(F)(F)C(F)(F)C(F)(F)F)c([N+](=O)[O-])c1</smiles>

White solid, 32.1mg, 26\%. (PE / EA = 3:1)

${ }^{1}$ H NMR (400 MHz, DMSO) $\delta 10.72(\mathrm{br}, 1 \mathrm{H}), 7.84-7.82(\mathrm{~m}, 1 \mathrm{H}), 7.88-7.80(\mathrm{~m}, 1 \mathrm{H}), 7.67$ (d, $J$ $=8.8 \mathrm{~Hz}, 1 \mathrm{H}), 2.09(\mathrm{~s}, 3 \mathrm{H})$.

${ }^{19}$ F NMR (376 MHz, DMSO) $\delta$-81.70 (3F), -105.30 (2F), -119.61 - -123.37 (10F), -125.75 $-129.79(2 \mathrm{~F})$.

${ }^{13}$ C NMR (101 MHz, DMSO) $\delta 169.75,149.30,144.30,130.23\left(\mathrm{t}, J_{C-F}=103.5 \mathrm{~Hz}\right), 120.85$, $113.55,24.10$.

HRMS Calcd for $\mathrm{C}_{16} \mathrm{H}_{7} \mathrm{~F}_{17} \mathrm{~N}_{2} \mathrm{O}_{3}\left[\mathrm{M}+\mathrm{H}^{+}\right]$: 621.0083 ; Found: 621.0094.

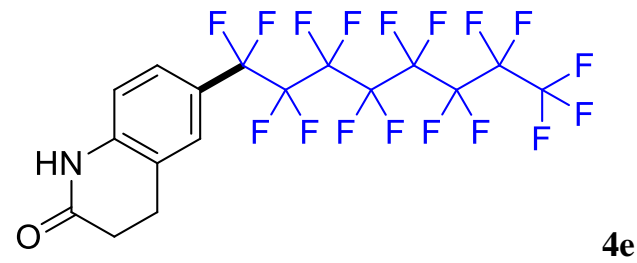

White solid, 69.0mg, 61\%. (PE / EA = 5:1)

${ }^{1}$ H NMR (400 MHz, DMSO) $\delta 10.44(\mathrm{br}, 1 \mathrm{H}), 7.32(\mathrm{~s}, 1 \mathrm{H}), 7.27(\mathrm{~d}, J=8.1 \mathrm{~Hz}, 1 \mathrm{H}), 6.96(\mathrm{~d}, J=$ $8.3 \mathrm{~Hz}, 1 \mathrm{H}), 2.93(\mathrm{~s}, 2 \mathrm{H}), 2.47(\mathrm{t}, J=6.5 \mathrm{~Hz}, 2 \mathrm{H})$.

${ }^{19}$ F NMR (376 MHz, DMSO) $\delta$-81.92 (3F), -107.46 - -111.86 (2F), -122.72 (10F), -127.11 (2F). ${ }^{13}$ C NMR (101 MHz, DMSO) $\delta 170.54,142.52,126.04\left(\mathrm{t}, J_{C-F}=6.4 \mathrm{~Hz}\right), 125.82\left(\mathrm{t}, J_{C-F}=6.0 \mathrm{~Hz}\right)$, $124.42,120.72\left(\mathrm{t}, J_{C-F}=24.0 \mathrm{~Hz}\right), 115.31,30.10,24.81$.

HRMS Calcd for $\mathrm{C}_{17} \mathrm{H}_{8} \mathrm{~F}_{17} \mathrm{NO}\left[\mathrm{M}+\mathrm{Na}^{+}\right]$: 588.0232; Found: 588.0221.<smiles>CC(=O)Nc1cc(C)c(C(F)(F)C(F)(F)C(F)(F)C(F)(F)C(F)(F)C(F)(F)C(F)(F)C(F)(F)F)cc1C</smiles>

White solid, 83.0mg, 69\%. (PE / EA = $5: 1$ )

${ }^{1}$ H NMR (400 MHz, DMSO) $\delta 9.38$ (br, 1H), 7.63 (s, 1H), 7.35 (s, 1H), 2.33 (s, 3H), 2.23 (s, 3H), $2.09(\mathrm{~s}, 3 \mathrm{H})$.

${ }^{19}$ F NMR (376 MHz, DMSO) $\delta$-80.21 (3F), -104.11 - -105.37 (2F), -118.48 - -124.69 (10F), $-125.39--126.45(2 \mathrm{~F})$.

${ }^{13}$ C NMR (101 MHz, DMSO) $\delta 168.66,140.37,135.11,129.77$ (t, $\left.J_{C-F}=9.5 \mathrm{~Hz}\right), 127.80,126.65$, 126.61, 23.41, 19.21, 17.14.

HRMS Calcd for $\mathrm{C}_{18} \mathrm{H}_{13} \mathrm{~F}_{17} \mathrm{NO}\left[\mathrm{M}+\mathrm{Na}^{+}\right]$: 604.0545; Found: 604.0553.

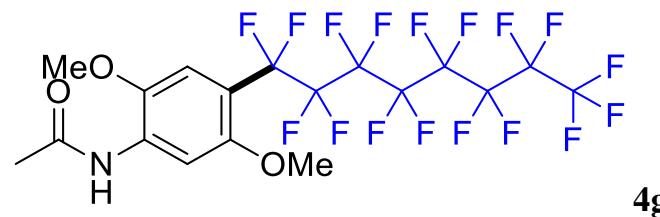

Red solid, 108.1, 88\%. (PE / EA = 5:1) 
${ }^{1}$ H NMR (400 MHz, DMSO) $\delta 9.36(\mathrm{br}, 1 \mathrm{H}), 8.14(\mathrm{~s}, 1 \mathrm{H}), 6.91(\mathrm{~s}, 1 \mathrm{H}), 3.74(\mathrm{~s}, 3 \mathrm{H}), 3.65(\mathrm{~s}, 3 \mathrm{H})$, $2.12(\mathrm{~s}, 3 \mathrm{H})$.

${ }^{19}$ F NMR (376 MHz, DMSO) $\delta-81.02(3 \mathrm{~F}),-104.83--107.91(2 \mathrm{~F}),-116.99--124.36(2 \mathrm{~F})$, $-125.52--127.72(2 \mathrm{~F})$.

${ }^{13}$ C NMR (101 MHz, DMSO) $\delta 169.48,152.30,142.21,133.02,110.41\left(\mathrm{t}, J_{C-F}=9.2 \mathrm{~Hz}\right), 105.92$, 56.29, 56.23, 24.13. (PE / EA = $5: 1$ )

HRMS Calcd for $\mathrm{C}_{18} \mathrm{H}_{12} \mathrm{~F}_{17} \mathrm{NO}_{3}\left[\mathrm{M}+\mathrm{H}^{+}\right]$: 614.0624; Found: 614.0613.<smiles>COc1cc(C(F)(F)C(F)(F)C(F)(F)C(F)(F)C(F)(F)C(F)(F)C(F)(F)C(F)(F)C(F)(F)F)ccc1NC(C)=O</smiles>

White solid, 96.3mg, 80\%. (PE / EA = 5:1)

${ }^{1}$ H NMR (400 MHz, DMSO) $\delta 9.40(\mathrm{br}, 1 \mathrm{H}), 8.28(\mathrm{~d}, J=8.4 \mathrm{~Hz}, 1 \mathrm{H}), 7.21-7.11(\mathrm{~m}, 2 \mathrm{H}), 3.87(\mathrm{~s}$, $3 \mathrm{H}), 2.13$ (s, 3H).

${ }^{19}$ F NMR (376 MHz, DMSO) $\delta-80.26(3 \mathrm{~F}),-106.01--110.38(2 \mathrm{~F}),-117.03--124.81(10 \mathrm{~F})$, $-125.33--126.82(2 \mathrm{~F})$.

${ }^{13}$ C NMR (101 MHz, DMSO) $\delta 169.21,148.93,131.67,120.79,119.27\left(\mathrm{t}, J_{C-F}=6.5 \mathrm{~Hz}\right), 108.78$, $56.19,24.04$

HRMS Calcd for $\mathrm{C}_{17} \mathrm{H}_{10} \mathrm{~F}_{17} \mathrm{NO}_{2}\left[\mathrm{M}+\mathrm{Na}^{+}\right]:$606.0338; Found: 606.0352<smiles>CC(=O)Nc1ccc(C(F)(F)C(F)(F)C(F)(F)C(F)(F)C(F)(F)C(F)(F)C(F)(F)C(F)(F)F)cc1O</smiles>

Red solid, 67.0mg, 51\%. (PE / EA = 5:1)

${ }^{1}$ H NMR (400 MHz, DMSO) $\delta 9.40(\mathrm{br}, 1 \mathrm{H}), 8.21(\mathrm{~d}, J=8.4 \mathrm{~Hz}, 1 \mathrm{H}), 7.47(\mathrm{~d}, J=7.3 \mathrm{~Hz}, 2 \mathrm{H})$, $7.35(\mathrm{t}, J=7.4 \mathrm{~Hz}, 2 \mathrm{H}), 7.27(\mathrm{t}, J=7.3 \mathrm{~Hz}, 1 \mathrm{H}), 7.20(\mathrm{~s}, 1 \mathrm{H}), 7.17(\mathrm{~d}, J=8.6 \mathrm{~Hz}, 1 \mathrm{H}), 5.30(\mathrm{~s}$, $2 \mathrm{H}), 2.15(\mathrm{~s}, 3 \mathrm{H})$.

${ }^{19}$ F NMR (376 MHz, DMSO) $\delta-81.73(3 \mathrm{~F}),-110.15(2 \mathrm{~F}),-120.61--125.39(10 \mathrm{~F}),-126.91(2 \mathrm{~F})$.

${ }^{13}$ C NMR (101 MHz, DMSO) $\delta 169.10,147.56,137.59$ (t, $\left.J_{C-F}=4.0 \mathrm{~Hz}\right), 132.31,128.25,127.77$, $127.42,121.97,120.41,119.13\left(\mathrm{t}, J_{C-F}=7.5 \mathrm{~Hz}\right), 110.71,70.35,23.76$.

HRMS Calcd for $\mathrm{C}_{23} \mathrm{H}_{14} \mathrm{~F}_{17} \mathrm{NO}_{2}\left[\mathrm{M}+\mathrm{H}^{+}\right]$: 660.0831; Found: 660.0851.

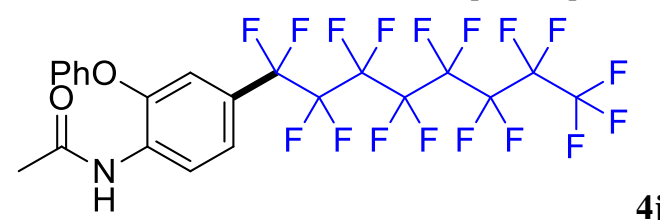

White solid, 71.0mg, 55\%. (PE / EA = 5: 1)

${ }^{1}$ H NMR (400 MHz, DMSO) $\delta 9.76(\mathrm{br}, 1 \mathrm{H}), 8.42-8.34(\mathrm{~m}, 1 \mathrm{H}), 7.41-7.37$ (m, 2H), $7.26-$ $7.24(\mathrm{~m}, 1 \mathrm{H}), 7.16-7.13(\mathrm{~m}, 1 \mathrm{H}), 7.06-6.98(\mathrm{~m}, 2 \mathrm{H}), 6.93(\mathrm{~d}, J=1.9 \mathrm{~Hz}, 1 \mathrm{H}), 2.11(\mathrm{~s}, 3 \mathrm{H})$.

${ }^{19}$ F NMR (376 MHz, DMSO) $\delta$-81.60 (3F), -110.48 (2F), -122.69 (10F), -126.89 (2F).

${ }^{13}$ C NMR (101 MHz, DMSO) $\delta 169.49,155.90,146.62,137.56,134.07,129.90,124.15,121.76(\mathrm{t}$, $\left.J_{C-F}=6.4 \mathrm{~Hz}\right), 120.00,118.90,115.42\left(\mathrm{t}, J_{C-F}=6.4 \mathrm{~Hz}\right), 23.66$.

HRMS Calcd for $\mathrm{C}_{22} \mathrm{H}_{12} \mathrm{~F}_{17} \mathrm{NO}_{2}\left[\mathrm{M}+\mathrm{Na}^{+}\right]$: 668.0494; Found: 668.0485 . 


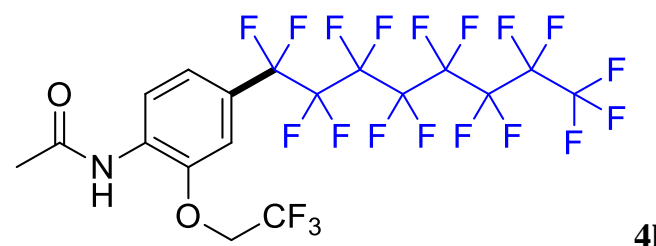

White solid, 63.8mg, 49\%. (PE / EA = 5:1)

${ }^{1}$ H NMR (400 MHz, DMSO) $\delta 9.32(\mathrm{br}, 1 \mathrm{H}), 8.18(\mathrm{~d}, J=8.5 \mathrm{~Hz}, 1 \mathrm{H}), 7.32(\mathrm{~s}, 1 \mathrm{H}), 7.18(\mathrm{dd}, J=$ $8.6,1.0 \mathrm{~Hz}, 1 \mathrm{H}), 4.80\left(\mathrm{q}, J_{H-F}=8.8 \mathrm{~Hz}, 2 \mathrm{H}\right), 2.10(\mathrm{~s}, 3 \mathrm{H})$.

${ }^{19}$ F NMR (376 MHz, DMSO) $\delta$-72.95 (3F), -81.92 (3F), -108.73 - -113.10 (2F), -119.76 -125.79 (10F), $-126.58--130.44(2 \mathrm{~F})$.

${ }^{13}$ C NMR (101 MHz, DMSO) $\delta 169.10,147.29,132.29,128.47,125.01,122.19\left(\mathrm{q}, J_{C-F}=10.0 \mathrm{~Hz}\right)$, $120.92\left(\mathrm{t}, J_{C-F}=6.0 \mathrm{~Hz}\right), 112.26,66.05\left(\mathrm{q}, J_{C-F}=34.6 \mathrm{~Hz}\right), 23.80$.

HRMS Calcd for $\mathrm{C}_{18} \mathrm{H}_{9} \mathrm{~F}_{1} \mathrm{NO}_{2}$ [M+Na $\left.{ }^{+}\right]$: 674.0206; Found: 674.0174.<smiles>CC(=O)Nc1ccc(C(F)(F)C(F)(F)C(F)(F)C(F)(F)C(F)(F)C(F)(F)C(F)(F)C(F)(F)F)cc1C</smiles>

White solid, 83.5mg, 71\%. (PE / EA = 5: 1)

${ }^{1}$ H NMR (400 MHz, DMSO) $\delta 9.34(\mathrm{br}, 1 \mathrm{H}), 7.79(\mathrm{~d}, J=8.6 \mathrm{~Hz}, 1 \mathrm{H}), 7.33(\mathrm{~s}, 1 \mathrm{H}), 7.30(\mathrm{~d}, J=$ $8.6 \mathrm{~Hz}, 1 \mathrm{H}), 2.19$ (s, 3H), 2.08 (s, 3H).

${ }^{19}$ F NMR (376 MHz, DMSO) $\delta-77.82--86.27$ (3F), -105.50 - -112.99 (2F), -118.01 - -124.81 (10F), $-126.17--128.79(2 \mathrm{~F})$.

${ }^{13}$ C NMR (101 MHz, DMSO) $\delta 169.11,140.96,131.33,131.15,128.59$ (t, $\left.J=6.1 \mathrm{~Hz}\right), 124.69(\mathrm{t}, J$ $=5.7 \mathrm{~Hz}), 124.03,23.93,18.04$.

HRMS Calcd for $\mathrm{C}_{17} \mathrm{H}_{10} \mathrm{~F}_{17} \mathrm{NO}\left[\mathrm{M}+\mathrm{Na}^{+}\right]$: 590.0383 ; Found: 590.0390 .<smiles>CCc1cc(C(F)(F)C(F)(F)C(F)(F)C(F)(F)C(F)(F)C(F)(F)C(F)(F)C(F)(F)C(F)(F)F)ccc1NC(C)=O</smiles>

White solid, $81.1 \mathrm{mg}, 70 \%$. (PE / EA = $5: 1$ )

${ }^{1}$ H NMR (400 MHz, DMSO) $\delta 9.38(\mathrm{br}, 1 \mathrm{H}), 7.80(\mathrm{~d}, J=8.5 \mathrm{~Hz}, 1 \mathrm{H}), 7.44-7.04(\mathrm{~m}, 2 \mathrm{H}), 2.68$ (q, $J=7.4 \mathrm{~Hz}, 2 \mathrm{H}), 2.10$ (s, 3H), $1.11(\mathrm{t}, J=7.5 \mathrm{~Hz}, 3 \mathrm{H})$.

${ }^{19}$ F NMR (376 MHz, DMSO) $\delta$-81.66 (3F), $-108.98--112.20$ (2F), -122.55 (10F), -126.88 (2F). ${ }^{13}$ C NMR (101 MHz, DMSO) $\delta 168.95,140.02,126.34\left(\mathrm{t}, J_{C-F}=6.8 \mathrm{~Hz}\right), 124.21,124.12,123.16$, $118.11,23.53,23.33,13.23$.

HRMS Calcd for $\mathrm{C}_{18} \mathrm{H}_{12} \mathrm{~F}_{17} \mathrm{NO}\left[\mathrm{M}+\mathrm{Na}^{+}\right]$: 604.0545; Found: 604.0538.<smiles>CC(=O)Nc1ccc(C(F)(F)C(F)(F)C(F)(F)C(F)(F)C(F)(F)C(F)(F)C(F)(F)C(F)(F)C(F)(F)F)cc1C(C)C</smiles>

White solid, 75.0mg, 63\%. (PE / EA = 5 : 1) 
${ }^{1}$ H NMR (400 MHz, DMSO) $\delta 9.42(\mathrm{br}, 1 \mathrm{H}), 7.74(\mathrm{~d}, J=8.3 \mathrm{~Hz}, 1 \mathrm{H}), 7.44(\mathrm{~s}, 1 \mathrm{H}), 7.29(\mathrm{~d}, J=$ $8.4 \mathrm{~Hz}, 1 \mathrm{H}), 3.43-3.35(\mathrm{~m}, 1 \mathrm{H}), 2.12(\mathrm{~s}, 3 \mathrm{H}), 1.20(\mathrm{~d}, J=3.1 \mathrm{~Hz}, 6 \mathrm{H})$.

${ }^{19}$ F NMR (376 MHz, DMSO) $\delta-80.89--84.46(3 \mathrm{~F}),-110.39--114.15(2 \mathrm{~F}),-123.01(10 \mathrm{~F})$, $-127.36(2 \mathrm{~F})$.

${ }^{13}$ C NMR (101 MHz, DMSO) $\delta 169.05,141.57,137.90,137.74\left(\mathrm{t}, J_{C-F}=6.2 \mathrm{~Hz}\right), 124.83\left(\mathrm{t}, J_{C-F}=\right.$ $4.0 \mathrm{~Hz}), 123.71\left(\mathrm{t}, J_{C-F}=5.8 \mathrm{~Hz}\right), 26.74,22.95,22.14$.

HRMS Calcd for $\mathrm{C}_{19} \mathrm{H}_{14} \mathrm{~F}_{17} \mathrm{NO}$ [M+Na+]: 618.0702; Found: 618.0692.<smiles>CC(=O)Nc1ccc(C(F)(F)C(F)(F)C(F)(F)C(F)(F)C(F)(F)C(F)(F)C(F)(F)C(F)(F)F)cc1C(C)(C)C</smiles>

White solid, 74.4mg, 61\%. (PE / EA = 5:1)

${ }^{1}$ H NMR (400 MHz, DMSO) $\delta 9.34(\mathrm{br}, 1 \mathrm{H}), 7.53(\mathrm{~s}, 1 \mathrm{H}), 7.42(\mathrm{dd}, J=8.4,1.5 \mathrm{~Hz}, 1 \mathrm{H}), 7.33(\mathrm{~d}$, $J=8.2 \mathrm{~Hz}, 1 \mathrm{H}), 2.06(\mathrm{~s}, 3 \mathrm{H}), 1.32(\mathrm{~s}, 9 \mathrm{H})$.

${ }^{19}$ F NMR (376 MHz, DMSO) $\delta-81.73$ (3F), -110.65 (2F), -122.60 (10F), $-126.93(2 \mathrm{~F})$.

${ }^{13}$ C NMR (101 MHz, DMSO) $\delta 169.60,141.26\left(\mathrm{t}, J_{C-F}=1.5 \mathrm{~Hz}\right), 140.79\left(\mathrm{t}, J_{C-F}=2.5 \mathrm{~Hz}\right), 137.81$, $132.51\left(\mathrm{t}, J_{C-F}=2.5 \mathrm{~Hz}\right), 124.91,124.76,35.08,30.25,29.41$.

HRMS Calcd for $\mathrm{C}_{20} \mathrm{H}_{16} \mathrm{~F}_{17} \mathrm{NO}$ [M+Na+]: 632.0858; Found: 632.0848 .

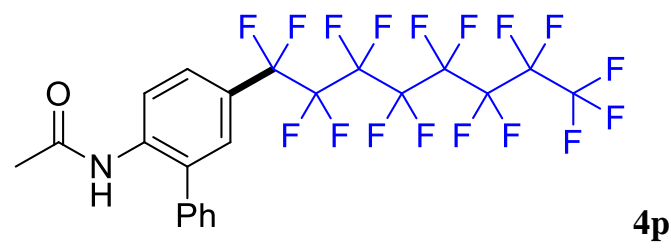

White solid, 78.1mg, 62\%. (PE / EA = 5:1)

${ }^{1}$ H NMR (400 MHz, DMSO) $\delta 9.44(\mathrm{br}, 1 \mathrm{H}), 7.89(\mathrm{~d}, J=8.1 \mathrm{~Hz}, 1 \mathrm{H}), 7.64(\mathrm{~d}, J=8.4 \mathrm{~Hz}, 1 \mathrm{H})$, $7.52-7.26(\mathrm{~m}, 6 \mathrm{H}), 1.94(\mathrm{~s}, 3 \mathrm{H})$.

${ }^{19}$ F NMR (376 MHz, DMSO) $\delta$-81.41 (3F), -110.31 (2F), -122.39 (10F), -126.66 (2F).

${ }^{13}$ C NMR (101 MHz, DMSO) $\delta 168.94,137.53,128.67,128.30\left(\mathrm{t}, J_{C-F}=7.0 \mathrm{~Hz}\right), 127.89,125.82$, $123.88,123.66,23.09$.

HRMS Calcd for $\mathrm{C}_{22} \mathrm{H}_{12} \mathrm{~F}_{17} \mathrm{NO}\left[\mathrm{M}+\mathrm{Na}^{+}\right]$: 652.0545; Found:652.0537.

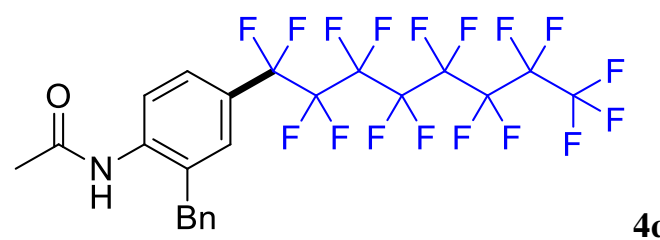

White solid, $81.1 \mathrm{mg}, 63 \%$. (PE / EA = 5:1)

${ }^{1} \mathrm{H}$ NMR $(400 \mathrm{MHz}, \mathrm{DMSO}) \delta 9.56(\mathrm{br}, 1 \mathrm{H}), 7.77(\mathrm{~d}, J=8.4 \mathrm{~Hz}, 1 \mathrm{H}), 7.48(\mathrm{dd}, J=8.4,0.6 \mathrm{~Hz}$, $1 \mathrm{H}), 7.40(\mathrm{~s}, 1 \mathrm{H}), 7.26(\mathrm{t}, J=7.3 \mathrm{~Hz}, 2 \mathrm{H}), 7.19-7.11(\mathrm{~m}, 3 \mathrm{H}), 4.09$ (s, 2H), $2.02(\mathrm{~s}, 3 \mathrm{H})$.

${ }^{19}$ F NMR (376 MHz, DMSO) $\delta-78.42--81.89$ (3F), -108.32 - -110.58 (2F), -121.18 - -122.65 (10F), $-125.26--127.40(2 \mathrm{~F})$.

${ }^{13}$ C NMR (101 MHz, DMSO) $\delta 168.63,140.42,137.52,128.60,128.24,126.07,124.70,124.68$, 124.64, 99.68, 36.39. 
HRMS Calcd for $\mathrm{C}_{23} \mathrm{H}_{14} \mathrm{~F}_{17} \mathrm{NO}$ [M+Na+]: 666.0702; Found: 666.0703.<smiles>CC(=O)Nc1ccc(C(F)(F)C(F)(F)C(F)(F)C(F)(F)C(F)(F)C(F)(F)C(F)(F)C(F)(F)F)cc1-c1ccc(Cl)cc1</smiles>

White solid, 54.4mg, 41\%. (PE / EA = 5:1)

${ }^{1} \mathbf{H}$ NMR $(400 \mathrm{MHz}, \mathrm{DMSO}) \delta 9.34(\mathrm{br}, 1 \mathrm{H}), 7.82(\mathrm{~d}, J=8.4 \mathrm{~Hz}, 1 \mathrm{H}), 7.41(\mathrm{~d}, J=8.8 \mathrm{~Hz}, 1 \mathrm{H})$, $7.34(\mathrm{~d}, J=8.5 \mathrm{~Hz}, 2 \mathrm{H}), 7.31(\mathrm{~s}, 1 \mathrm{H}), 7.25(\mathrm{~d}, J=8.5 \mathrm{~Hz}, 2 \mathrm{H}), 1.89$ (s, 3H).

${ }^{19}$ F NMR (376 MHz, DMSO) $\delta$-82.24 (3F), -110.44 (2F), -118.75 - -125.51 (10F), -127.24 (2F). ${ }^{13}$ C NMR (101 MHz, DMSO) $\delta$ 168.85, 138.99, 136.20, 134.11, 132.79, 130.33, 128.48, $128.02(\mathrm{t}$, $\left.J_{C-F}=5.5 \mathrm{~Hz}\right), 126.01,125.69,99.59,23.21$.

HRMS Calcd for $\mathrm{C}_{22} \mathrm{H}_{11} \mathrm{~F}_{17} \mathrm{NOCI}\left[\mathrm{M}+\mathrm{Na}^{+}\right]$: 686.0155; Found: 686.0142 .<smiles>CC(=O)Nc1ccc(C(F)(F)C(F)(F)C(F)(F)C(F)(F)C(F)(F)C(F)(F)C(F)(F)C(F)(F)F)cc1Cl</smiles>

White solid, 50.4mg, 43\%. (PE / EA = $5: 1$ )

${ }^{1}$ H NMR (400 MHz, DMSO) $\delta 9.28(\mathrm{br}, 1 \mathrm{H}), 7.44(\mathrm{~d}, J=8.5 \mathrm{~Hz}, 1 \mathrm{H}), 7.37(\mathrm{~s}, 1 \mathrm{H}), 7.27-7.19(\mathrm{~m}$, $1 \mathrm{H}), 2.16(\mathrm{~s}, 3 \mathrm{H})$.

${ }^{19}$ F NMR (376 MHz, DMSO) $\delta$-82.28 (3F), -105.76 (2F), -120.84 - -124.18 (10F), -127.24 (2F).

${ }^{13}$ C NMR $\left(101 \mathrm{MHz}, \mathrm{CDCl}_{3}\right) \delta 168.77,143.90,137.81,137.65,137.59,126.57\left(\mathrm{t}, J_{C-F}=8.0 \mathrm{~Hz}\right)$, $124.78\left(\mathrm{t}, J_{C-F}=3.6 \mathrm{~Hz}\right), 29.24$.

HRMS Calcd for $\mathrm{C}_{16} \mathrm{H}_{7} \mathrm{~F}_{17} \mathrm{NOCI}\left[\mathrm{M}+\mathrm{Na}^{+}\right]$: 609.9842; Found: 609.9830.<smiles>CC(C)(C)C(=O)Nc1ccc(C(F)(F)C(F)(F)C(F)(F)C(F)(F)C(F)(F)C(F)(F)C(F)(F)C(F)(F)F)cc1</smiles>

White solid, 72.7mg, 61\%. (PE / EA = 5:1)

${ }^{1} \mathbf{H}$ NMR $\left(400 \mathrm{MHz}, \mathrm{CDCl}_{3}\right) \delta 7.69(\mathrm{~d}, J=8.6 \mathrm{~Hz}, 2 \mathrm{H}), 7.56(\mathrm{br}, 1 \mathrm{H}), 7.51(\mathrm{~d}, J=8.7 \mathrm{~Hz}, 2 \mathrm{H})$, $1.38(\mathrm{~s}, 9 \mathrm{H})$.

${ }^{19} \mathbf{F}$ NMR (376 MHz, $\left.\mathrm{CDCl}_{3}\right) \delta-82.32(3 \mathrm{~F}),-111.07--112.00(2 \mathrm{~F}),-121.23--124.59(10 \mathrm{~F})$, $-127.29(2 \mathrm{~F})$.

${ }^{13}$ C NMR $\left(101 \mathrm{MHz}, \mathrm{CDCl}_{3}\right) \delta 177.31,142.28,128.05\left(\mathrm{t}, J_{C-F}=6.5 \mathrm{~Hz}\right), 119.31,40.15,27.31$.

HRMS Calcd for $\mathrm{C}_{19} \mathrm{H}_{14} \mathrm{~F}_{17} \mathrm{NO}$ [M+H'] 596.0882 ; Found: 596.0871 . 


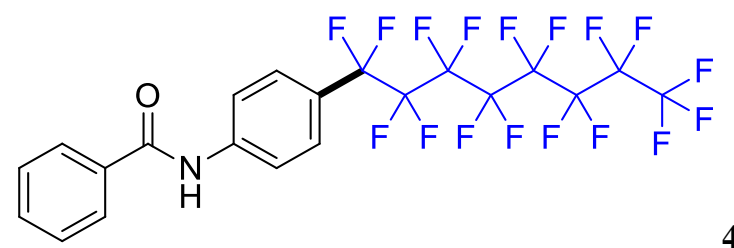

White solid, 52.9mg, 43\%. (PE / EA = 5:1)

${ }^{1}$ H NMR (400 MHz, DMSO) $\delta 10.49(\mathrm{br}, 1 \mathrm{H}), 8.02(\mathrm{~d}, J=8.7 \mathrm{~Hz}, 2 \mathrm{H}), 7.97$ (d, $\left.J=7.1 \mathrm{~Hz}, 2 \mathrm{H}\right)$, $7.62(\mathrm{t}, J=7.3 \mathrm{~Hz}, 1 \mathrm{H}), 7.57-7.53(\mathrm{~m}, 2 \mathrm{H}), 7.50(\mathrm{~d}, J=8.8 \mathrm{~Hz}, 2 \mathrm{H})$.

${ }^{19}$ F NMR (376 MHz, DMSO) $\delta-82.42$ (3F), -110.56 - -111.57 (2F), -123.01 (10F), -126.93 $-127.83(2 \mathrm{~F})$.

${ }^{13}$ C NMR (101 MHz, DMSO) $\delta$ 165.95, 143.54, 137.82, 134.66, 131.63, 128.14, 127.73, 126.84 (t, $\left.J_{C-F}=6.2 \mathrm{~Hz}\right), 119.29$.

HRMS Calcd for $\mathrm{C}_{21} \mathrm{H}_{10} \mathrm{~F}_{17} \mathrm{NO}\left[\mathrm{M}+\mathrm{H}^{+}\right]$: 616.0569; Found: 616.0570 .

6. Transformations and applications
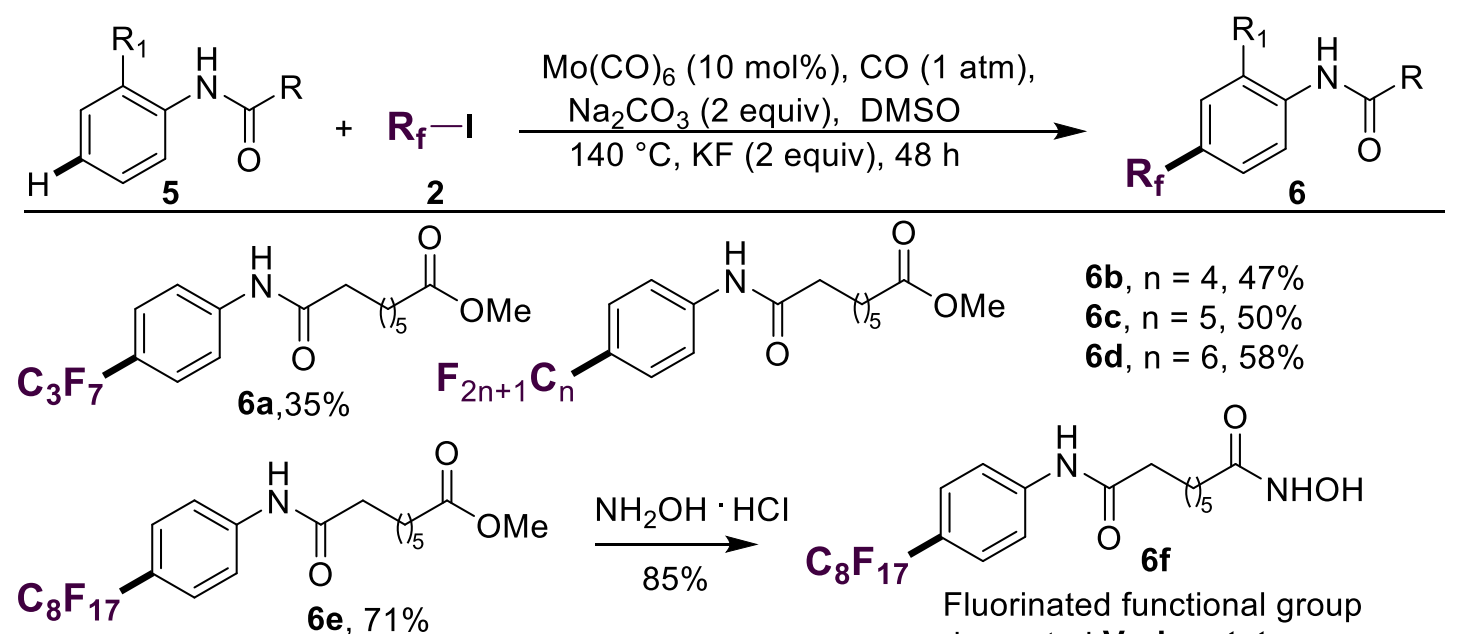<smiles>C=C(NO)NCC(=O)Nc1ccc([I+]#[P-])cc1</smiles>

Fluorinated functional group

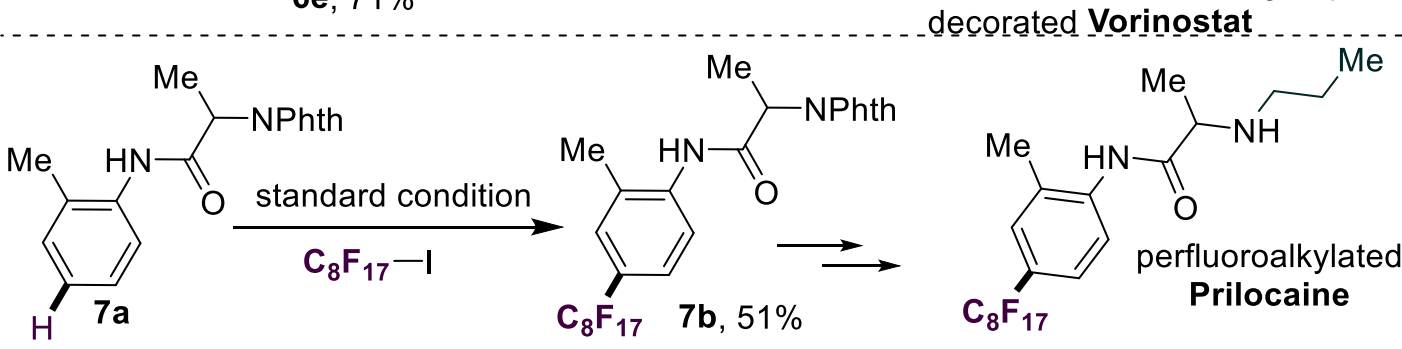

General Procedures As Above.<smiles>COC(=O)CCCCCCC(=O)Nc1ccc(C(F)(F)C(F)(F)C(F)(F)F)cc1</smiles>

White solid, 30.2mg, 35\%. (PE / EA = 3:1)

${ }^{1}$ H NMR (400 MHz, DMSO) $\delta 10.26(\mathrm{br}, 1 \mathrm{H}), 7.83(\mathrm{~d}, J=8.7 \mathrm{~Hz}, 2 \mathrm{H}), 7.59$ (d, $\left.J=8.7 \mathrm{~Hz}, 2 \mathrm{H}\right)$, $3.57(\mathrm{~s}, 3 \mathrm{H}), 2.35-2.27(\mathrm{~m}, 4 \mathrm{H}), 1.63-1.48(\mathrm{~m}, 4 \mathrm{H}), 1.29(\mathrm{t}, J=3.5 \mathrm{~Hz}, 4 \mathrm{H})$.

${ }^{19}$ F NMR (376 MHz, DMSO) $\delta-79.53$ (3F), -109.94 (2F), -126.20 (2F). 
${ }^{13}$ C NMR (101 MHz, DMSO) $\delta$ 173.36, 171.99, 143.15, 128.64, $127.40\left(\mathrm{t}, J_{C-F}=6.4 \mathrm{~Hz}\right), 118.88$, $51.18,36.41,33.23,28.28,28.22,24.77,24.32$.

HRMS Calcd for $\mathrm{C}_{18} \mathrm{H}_{20} \mathrm{~F}_{7} \mathrm{NO}_{3}\left[\mathrm{M}+\mathrm{H}^{+}\right]$: 432.1410; Found: 432.1396.<smiles>COC(=O)CCCCCCC(=O)Nc1ccc(C(F)(F)C(F)(F)C(F)(F)C(F)(F)F)cc1</smiles>

White solid, 45.2mg, 47\%. (PE / EA = $3: 1$ )

${ }^{1}$ H NMR (400 MHz, DMSO) $\delta 10.25(\mathrm{br}, 1 \mathrm{H}), 7.83(\mathrm{~d}, J=8.7 \mathrm{~Hz}, 2 \mathrm{H}), 7.59(\mathrm{~d}, J=8.7 \mathrm{~Hz}, 2 \mathrm{H})$, 3.57 (s, 3H), $2.35-2.26(\mathrm{~m}, 4 \mathrm{H}), 1.62-1.47(\mathrm{~m}, 4 \mathrm{H}), 1.33-1.24(\mathrm{~m}, 4 \mathrm{H})$.

${ }^{19}$ F NMR (376 MHz, DMSO) $\delta-80.64$ (3F), -109.26 (2F), -122.57 (2F), -124.31 - -126.32 (2F).

${ }^{13}$ C NMR (101 MHz, DMSO) $\delta 173.31,171.95,143.12,127.44\left(\mathrm{t}, J_{C-F}=6.4 \mathrm{~Hz}\right), 120.73\left(\mathrm{t}, J_{C-F}=\right.$ $24.3 \mathrm{~Hz}), 118.80,51.09,36.35,33.17,28.22,28.17,24.71,24.25$.

HRMS Calcd for $\mathrm{C}_{19} \mathrm{H}_{20} \mathrm{~F}_{9} \mathrm{NO}_{3}$ [M+Na $]$ : 504.1197; Found: 504.1207.<smiles>COC(=O)CCCCCCC(=O)Nc1ccc(C(F)(F)C(F)(F)C(F)(F)C(F)(F)C(F)(F)F)cc1</smiles>

White solid, 53.0mg, 50\%. (PE / EA = $3: 1$ )

${ }^{1} \mathrm{H}$ NMR (400 MHz, DMSO) $\delta 10.26(\mathrm{br}, 1 \mathrm{H}), 7.83(\mathrm{~d}, J=8.7 \mathrm{~Hz}, 2 \mathrm{H}), 7.59(\mathrm{~d}, J=8.7 \mathrm{~Hz}, 2 \mathrm{H})$, 3.57 (s, 3H), $2.36-2.27(\mathrm{~m}, 4 \mathrm{H}), 1.64-1.46(\mathrm{~m}, 4 \mathrm{H}), 1.29(\mathrm{t}, J=3.5 \mathrm{~Hz}, 4 \mathrm{H})$.

${ }^{19}$ F NMR (376 MHz, DMSO) $\delta-78.80--82.35$ (3F), -107.41 - -110.57 (2F), -118.42 - -124.39 (4F), $-125.15--128.09(2 \mathrm{~F})$.

${ }^{13}$ C NMR (101 MHz, DMSO) $\delta 173.79,172.42,143.59,127.93\left(\mathrm{t}, J_{C-F}=5.9 \mathrm{~Hz}\right), 121.26\left(\mathrm{t}, J_{C-F}=\right.$ $24.5 \mathrm{~Hz}), 119.28,51.59,36.84,33.66,28.70,28.65,25.20,24.74$.

HRMS Calcd for $\mathrm{C}_{20} \mathrm{H}_{20} \mathrm{~F}_{11} \mathrm{NO}_{3}\left[\mathrm{M}+\mathrm{Na}^{+}\right]$: 554.1165; Found: 554.1146.<smiles>COC(=O)CCCCCCC(=O)Nc1ccc(C(F)(F)C(F)(F)C(F)(F)C(F)(F)C(F)(F)C(F)(F)F)cc1</smiles>

White solid, 67.2mg, 58\%. (PE / EA = $3: 1$ )

${ }^{1}$ H NMR (400 MHz, DMSO) $\delta 10.25(\mathrm{br}, 1 \mathrm{H}), 7.82(\mathrm{~d}, J=8.8 \mathrm{~Hz}, 2 \mathrm{H}), 7.56(\mathrm{~d}, J=8.8 \mathrm{~Hz}, 2 \mathrm{H})$, $3.57(\mathrm{~d}, J=8.0 \mathrm{~Hz}, 3 \mathrm{H}), 2.34-2.25(\mathrm{~m}, 4 \mathrm{H}), 1.61-1.47(\mathrm{~m}, 4 \mathrm{H}), 1.33-1.25(\mathrm{~m}, 4 \mathrm{H})$.

${ }^{19}$ F NMR (376 MHz, DMSO) $\delta-78.56--83.19$ (3F), -107.69 - -110.83 (2F), -117.31 - -125.17 $(6 \mathrm{~F}),-125.53--128.10(2 \mathrm{~F})$.

${ }^{13}$ C NMR (101 MHz, DMSO) $\delta 173.44,172.08,143.20,127.50\left(\mathrm{t}, J_{C-F}=6.5 \mathrm{~Hz}\right), 120.96\left(\mathrm{t}, J_{C-F}=\right.$ $24.4 \mathrm{~Hz}), 118.89,51.21,36.46,33.28,28.32,28.27,24.83,24.36$.

HRMS Calcd for $\mathrm{C}_{21} \mathrm{H}_{20} \mathrm{~F}_{13} \mathrm{NO}_{3}$ [M+Na ${ }^{+}$]: 604.1133; Found: 604.1124. 
<smiles>COC(=O)CCCCCCC(=O)Nc1ccc(C(F)(F)C(F)(F)C(F)(F)C(F)(F)C(F)(F)C(F)(F)C(F)(F)C(F)(F)F)cc1</smiles>

White solid, 96.3mg, 71\%. (PE / EA = 3:1)

${ }^{1} \mathbf{H}$ NMR $(400 \mathrm{MHz}, \mathrm{DMSO}) \delta 10.16(\mathrm{br}, 1 \mathrm{H}), 7.70(\mathrm{~d}, J=8.7 \mathrm{~Hz}, 2 \mathrm{H}), 7.37(\mathrm{~d}, J=8.7 \mathrm{~Hz}, 2 \mathrm{H})$, $3.56(\mathrm{~s}, 3 \mathrm{H}), 2.33-2.24(\mathrm{~m}, 4 \mathrm{H}), 1.61-1.53(\mathrm{~m}, 4 \mathrm{H}), 1.35-1.30(\mathrm{~m}, 4 \mathrm{H})$.

${ }^{19}$ F NMR (376 MHz, DMSO) $\delta$-82.19 (3F), $-110.68(2 \mathrm{~F}),-122.98(10 \mathrm{~F}),-127.25(2 \mathrm{~F})$.

${ }^{13}$ C NMR (101 MHz, DMSO) $\delta 173.33,171.89,143.35,126.87,121.26\left(\mathrm{t}, J_{C-F}=24.8 \mathrm{~Hz}\right), 118.12$, $50.69,36.49,33.26,28.53,28.51,24.83,24.48$.

HRMS Calcd for $\mathrm{C}_{23} \mathrm{H}_{20} \mathrm{~F}_{17} \mathrm{NO}_{3}\left[\mathrm{M}+\mathrm{Na}^{+}\right]$]: 704.1069; Found: 704.1057.

Procedures for hydroxylamination according to reported methods. [Journal of Medicinal Chemistry, 45(13), 2877-2885; 2002]<smiles>O=C(CCCCCCC(=O)Nc1ccc(C(F)(F)C(F)(F)C(F)(F)C(F)(F)C(F)(F)C(F)(F)C(F)(F)C(F)(F)F)cc1)NO</smiles>

White solid, $112.0 \mathrm{mg}, 85 \%$.

${ }^{1}$ H NMR (400 MHz, DMSO) $\delta 10.38(\mathrm{br}, 1 \mathrm{H}), 7.72(\mathrm{~d}, J=7.6 \mathrm{~Hz}, 2 \mathrm{H}), 7.34(\mathrm{~d}, J=7.7 \mathrm{~Hz}, 2 \mathrm{H})$, $2.36-2.19(\mathrm{~m}, 4 \mathrm{H}), 1.56-1.54(\mathrm{~m}, 4 \mathrm{H}), 1.40-1.25(\mathrm{~m}, 4 \mathrm{H})$.

${ }^{19}$ F NMR (376 MHz, DMSO) $\delta-82.17$ (3F), -110.67 (2F), -119.09 - -125.77 (10F), -127.26 (2F).

${ }^{13}$ C NMR (101 MHz, DMSO) $\delta$ 173.21, 171.84, 143.30, $137.49(\mathrm{t}, J=5.0 \mathrm{~Hz}), 126.69,121.05(\mathrm{t}$, $\left.J_{C-F}=24.4 \mathrm{~Hz}\right), 118.05,36.34,33.17,33.15,28.39,24.72,24.36$.

HRMS Calcd for $\mathrm{C}_{22} \mathrm{H}_{19} \mathrm{~F}_{17} \mathrm{NO}_{3}\left[\mathrm{M}+\mathrm{Na}^{+}\right]$: 682.1124; Found: 682.1113 .

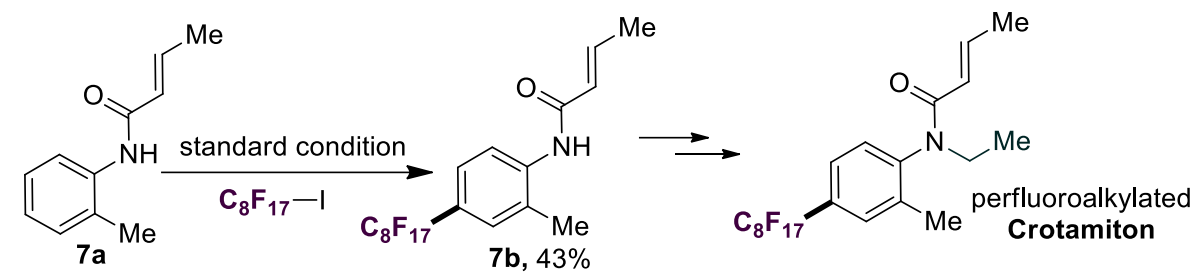

General Procedures As Above.

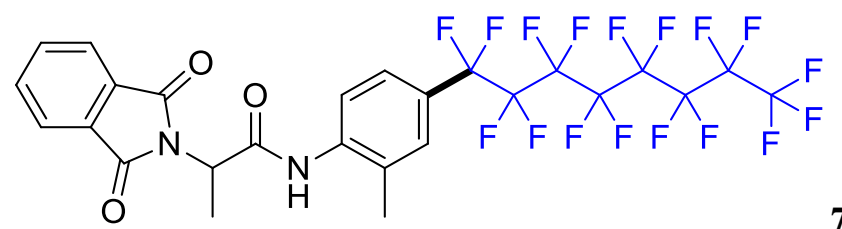

White solid, 73.8mg, 51\%. (PE / EA = 2:1)

${ }^{1}$ H NMR (400 MHz, DMSO) $\delta 9.67$ (br, 1H), $7.92-7.84(\mathrm{~m}, 4 \mathrm{H}), 7.51(\mathrm{~d}, J=8.3 \mathrm{~Hz}, 1 \mathrm{H}), 7.48-$ $7.38(\mathrm{~m}, 2 \mathrm{H}), 5.01(\mathrm{q}, J=7.1 \mathrm{~Hz}, 1 \mathrm{H}), 2.21(\mathrm{~s}, 3 \mathrm{H}), 1.62(\mathrm{~d}, J=7.2 \mathrm{~Hz}, 3 \mathrm{H})$.

${ }^{19}$ F NMR (377 MHz, DMSO) $\delta-80.80(3 \mathrm{~F}),-109.50(2 \mathrm{~F}),-118.67--141.45(12 \mathrm{~F})$.

${ }^{13}$ C NMR (101 MHz, DMSO) $\delta 167.98,167.46,140.15,134.49,133.87,131.85,128.38(\mathrm{t}, J=7.1$ $\mathrm{Hz}), 126.18,124.37$ (t, $J=16.5 \mathrm{~Hz}), 124.06,123.13,48.49,17.61,15.13$. 
HRMS Calcd for $\mathrm{C}_{26} \mathrm{H}_{15} \mathrm{~F}_{17} \mathrm{~N}_{2} \mathrm{O}_{3}\left[\mathrm{M}+\mathrm{Na}^{+}\right]$: 749.0709; Found: 749.0715. 


\section{Transformations}

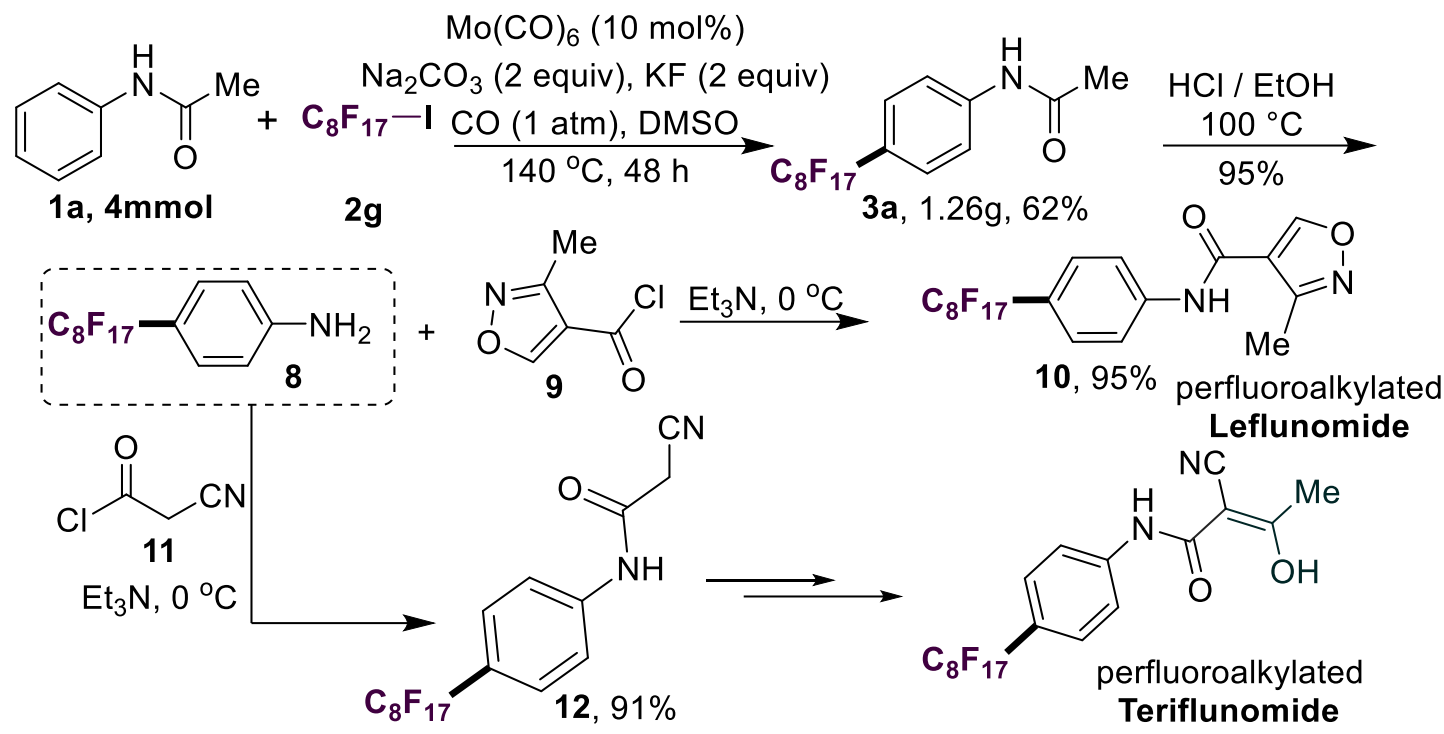

Step for gram scale reaction: A mixture of $\mathbf{1}$ ( 4 mmol, 1.0 equiv), $\mathbf{2 h}$ (3 equiv), $\mathrm{Mo}(\mathrm{CO})_{6}$ (10 mmol \%), $\mathrm{Na}_{2} \mathrm{CO}_{3}$ (2 equiv), $\mathrm{KF}(30 \mathrm{mmol} \%$ ) and DMSO $(30 \mathrm{~mL})$ in a $100 \mathrm{~mL}$ glass bottle sealed under $\mathrm{CO}$ atmosphere was heated at $140{ }^{\circ} \mathrm{C}$ for 48 hours. The reaction mass was extracted in EA $(25 \mathrm{~mL} \times 3)$. The combined organic layer was washed with water $(100 \mathrm{~mL})$, dried over sodium sulfate, and concentrated under reduced pressure, purified by column chromatography (PE / EA = $5: 1)$ on silica gel to give the product $4 \mathbf{a}(1.26 \mathrm{~g}, 62 \%)$.

Step for the hydrolysis of amide: To a $100 \mathrm{~mL}$ glass bottle, a mixture of $\mathbf{4 a}(1.0 \mathrm{~g}$, lequiv) in ethanol $(20 \mathrm{~mL}), 1.5 \mathrm{ml}$ concentrated $\mathrm{HCl}$ was added, then heated to reflux for $1 \mathrm{~h}$. The mixture was cooled to room temperature, $20 \mathrm{~mL} \mathrm{H} \mathrm{H}_{2} \mathrm{O}$ was added, then $\mathrm{NaHCO}_{3}$ was added slowly. Extraction with $\mathrm{CH}_{2} \mathrm{Cl}_{2}$, dried over sodium sulfate, purified by column chromatography (PE / EA $=10: 1)$ on silica gel to give the product 8 .

Step for amidation: A mixture of $8\left(0.2 \mathrm{mmol}, 1\right.$ equiv) and $\mathrm{Et}_{3} \mathrm{~N}$ ( 1 equiv) in $1 \mathrm{~mL} \mathrm{CH}_{2} \mathrm{Cl}_{2}, \mathbf{9}$ or 11 (1.5 equiv) was added slowly at $0{ }^{\circ} \mathrm{C}$. After the reaction completely, extracted with $\mathrm{CH}_{2} \mathrm{Cl}_{2}$, dried over sodium sulfate, purified by column chromatography (PE / EA $=10: 1)$ on silica gel to give the product 10 or 12 .<smiles>Nc1ccc(C(F)(F)C(F)(F)C(F)(F)C(F)(F)C(F)(F)C(F)(F)C(F)(F)C(F)(F)C(F)(F)F)cc1</smiles>

Lavender solid, $0.84 \mathrm{~g}, 95 \%$. (PE / EA $=10: 1$ )

${ }^{1} \mathbf{H}$ NMR $\left(400 \mathrm{MHz}, \mathrm{CDCl}_{3}\right) \delta 7.34(\mathrm{~d}, J=8.6 \mathrm{~Hz}, 2 \mathrm{H}), 6.71(\mathrm{~d}, J=8.6 \mathrm{~Hz}, 2 \mathrm{H}), 3.89(\mathrm{~b}, 2 \mathrm{H})$.

${ }^{19}$ F NMR $\left(376 \mathrm{MHz}, \mathrm{CDCl}_{3}\right) \delta-80.79(3 \mathrm{~F}),-105.41--112.55(2 \mathrm{~F}),-121.30--122.74(10 \mathrm{~F})$, $-125.04--127.57(2 \mathrm{~F})$.

${ }^{13}$ C NMR $\left(101 \mathrm{MHz}, \mathrm{CDCl}_{3}\right) \delta 149.72,128.41\left(\mathrm{t}, J_{C-F}=6.6 \mathrm{~Hz}\right), 118.17\left(\mathrm{t}, J_{C-F}=24.9 \mathrm{~Hz}\right)$, 114.34 .

HRMS Calcd for $\mathrm{C}_{14} \mathrm{H}_{6} \mathrm{~F}_{17} \mathrm{~N}$ [M+H+ $\mathrm{H}^{+}$]: 512.0287; Found: 512.0293 . 


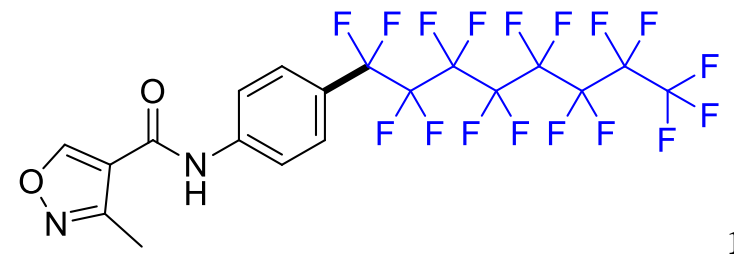

White solid, 117.8mg, 95\%. (PE / EA = 5:1)

${ }^{1}$ H NMR (400 MHz, DMSO) $\delta 10.32(\mathrm{br}, 1 \mathrm{H}), 9.05(\mathrm{~s}, 1 \mathrm{H}), 8.01(\mathrm{~d}, J=73.9 \mathrm{~Hz}, 2 \mathrm{H}), 7.48(\mathrm{~d}, J=$ $102.7 \mathrm{~Hz}, 2 \mathrm{H}), 2.67$ (s, 3H).

${ }^{19}$ F NMR (377 MHz, DMSO) $\delta$-80.60 (3F), -109.59 (2F), -123.02 (12F).

${ }^{13}$ C NMR (101 MHz, DMSO) $\delta 173.34,159.63$ (t, $\left.J_{C-F}=7.0 \mathrm{~Hz}\right), 148.94,139.43,129.83,127.48$, $119.90,111.67,12.11$.

HRMS Calcd for $\mathrm{C}_{19} \mathrm{H}_{9} \mathrm{~F}_{17} \mathrm{~N}_{2} \mathrm{O}_{2}$ [M+Na+ ${ }^{+}$: 643.0290; Found: 643.0279.

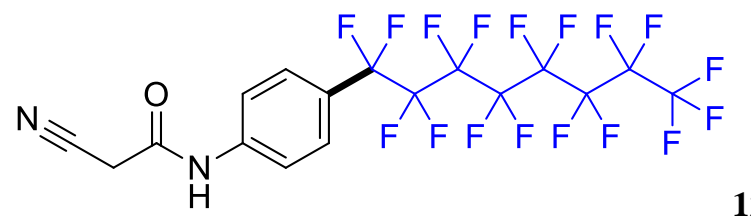

White solid, 105.3mg, 91\%. (PE / EA = $3: 1)$

${ }^{1} \mathrm{H}$ NMR (400 MHz, DMSO) $\delta 10.46(\mathrm{br}, 1 \mathrm{H}), 7.62(\mathrm{~d}, J=8.8 \mathrm{~Hz}, 2 \mathrm{H}), 7.46(\mathrm{~d}, J=8.6 \mathrm{~Hz}, 2 \mathrm{H})$, $3.90(\mathrm{~s}, 2 \mathrm{H})$.

${ }^{19}$ F NMR (376 MHz, DMSO) $\delta-78.80--82.40(3 \mathrm{~F}),-116.18--118.52(2 \mathrm{~F}),-119.05--124.02$ (10F), $-125.52--128.74(2 \mathrm{~F})$.

${ }^{13}$ C NMR (101 MHz, DMSO) $\delta 161.37,139.67,129.05,118.60,115.80,50.72,26.84$.

HRMS Calcd for $\mathrm{C}_{17} \mathrm{H}_{7} \mathrm{~F}_{17} \mathrm{~N}_{2} \mathrm{O}$ [M+Na+ ${ }^{+}$: 601.0185; Found: 601.0188 .

\section{NOESY analysis of $4 a$}

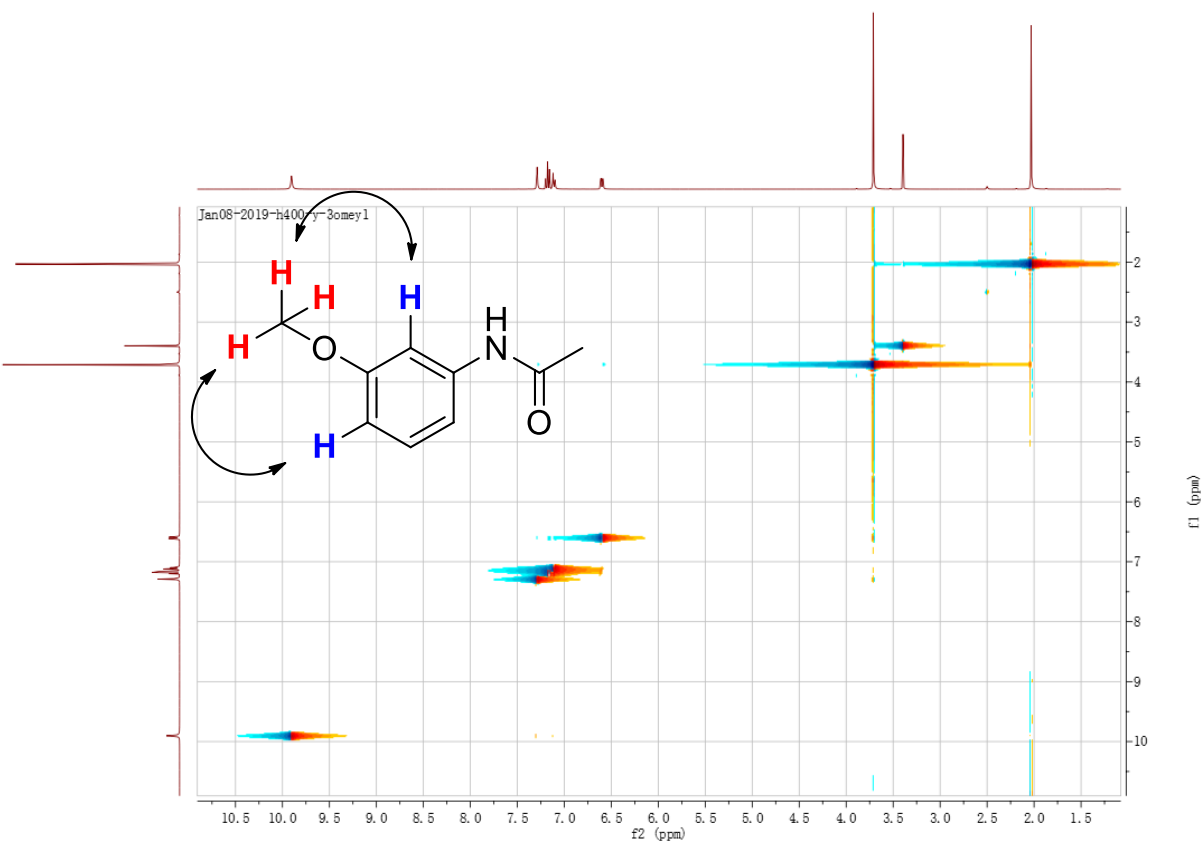



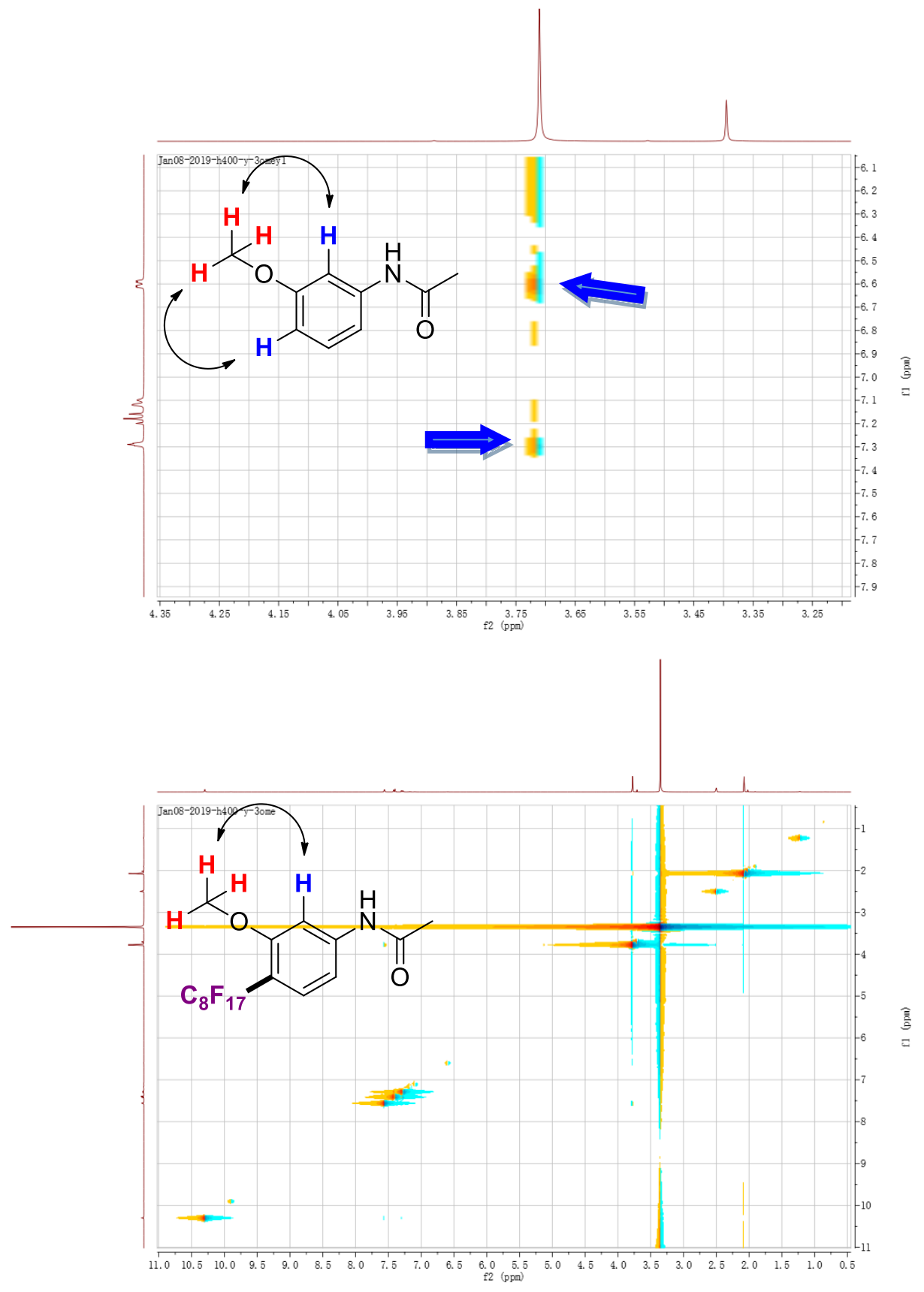


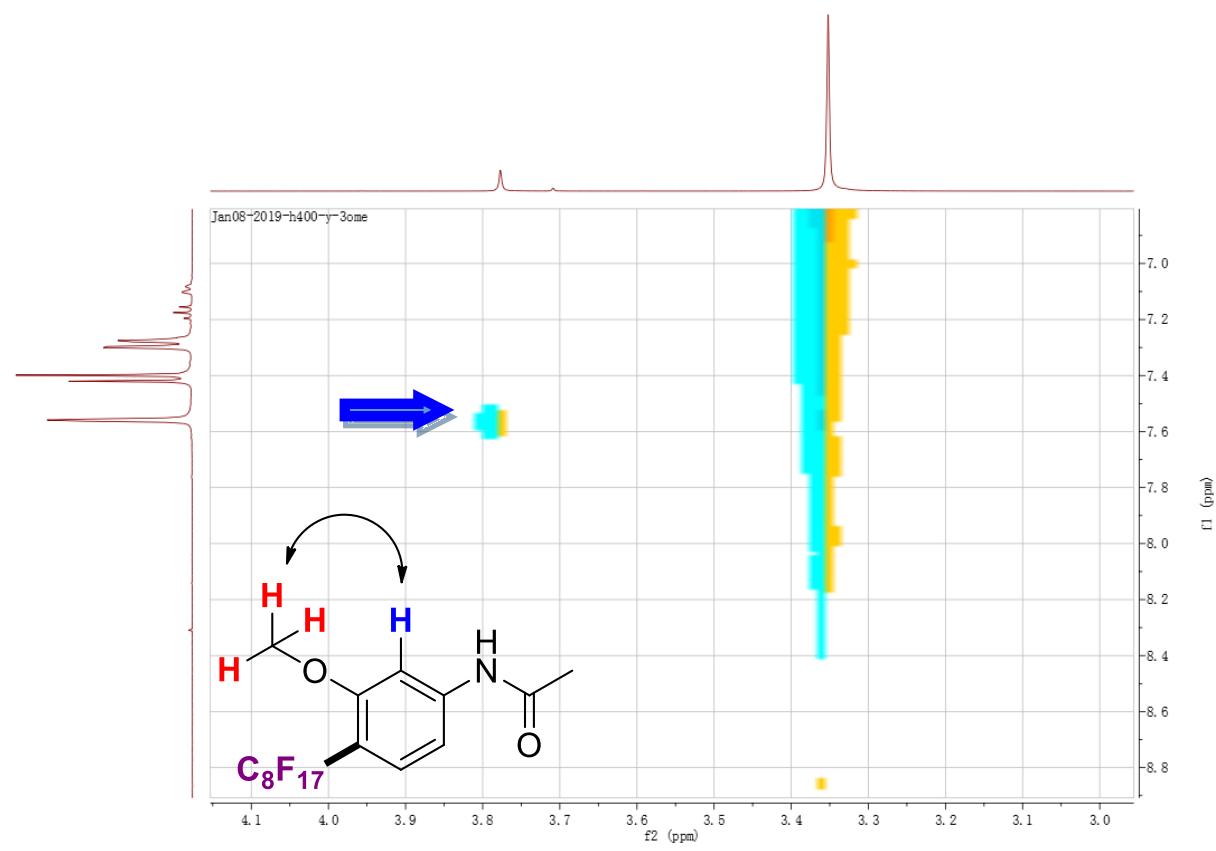

9. Preliminary mechanism studies

a)Raidical trapping experiments

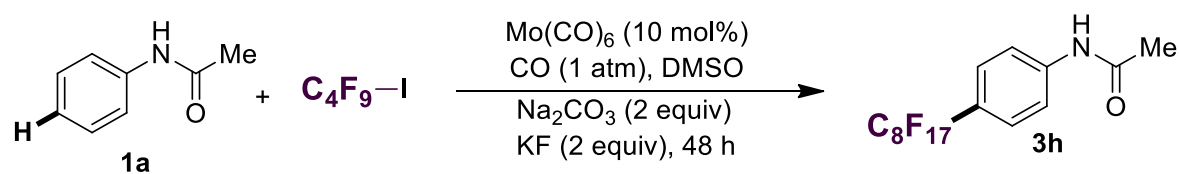

TEMPO, 1 equiv, $3 \mathbf{h}, 35 \% \quad \mathrm{BHT}, \quad 3$ equiv, $3 \mathrm{~h}$, trace

General Procedures: A mixture of $\mathbf{1 a}\left(0.2 \mathrm{mmol}, 1.0\right.$ equiv), $\mathrm{IC}_{4} \mathrm{~F}_{9}$ ( 3 equiv), $\mathrm{Mo}(\mathrm{CO})_{6}(5.3 \mathrm{mg}$, $10 \mathrm{mmol} \%$ ), $\mathrm{Na}_{2} \mathrm{CO}_{3}$ (41.0 mg, 2 equiv), $\mathrm{KF}$ ( $23.2 \mathrm{mg}, 30 \mathrm{mmol} \%$ ), TEMPO or BHT (1 equiv or 3 equiv) and DMSO $(0.5 \mathrm{~mL})$ in a $15 \mathrm{~mL}$ glass vial sealed under $\mathrm{CO}$ atmosphere was heated at 140 ${ }^{\circ} \mathrm{C}$ for 48 hours. The reaction mixture cooled to room temperature and concentrated in vacuo. The resulting residue was purified by column chromatography (PE / EA $=5: 1$ ) on silica gel to give the product. Analyzed by ${ }^{1} \mathrm{H}$ NMR and TLC.

Radicals captured through BHT analyzed by Crude ${ }^{19} \mathrm{~F}$ NMR and ${ }^{13} \mathrm{C}$ NMR.

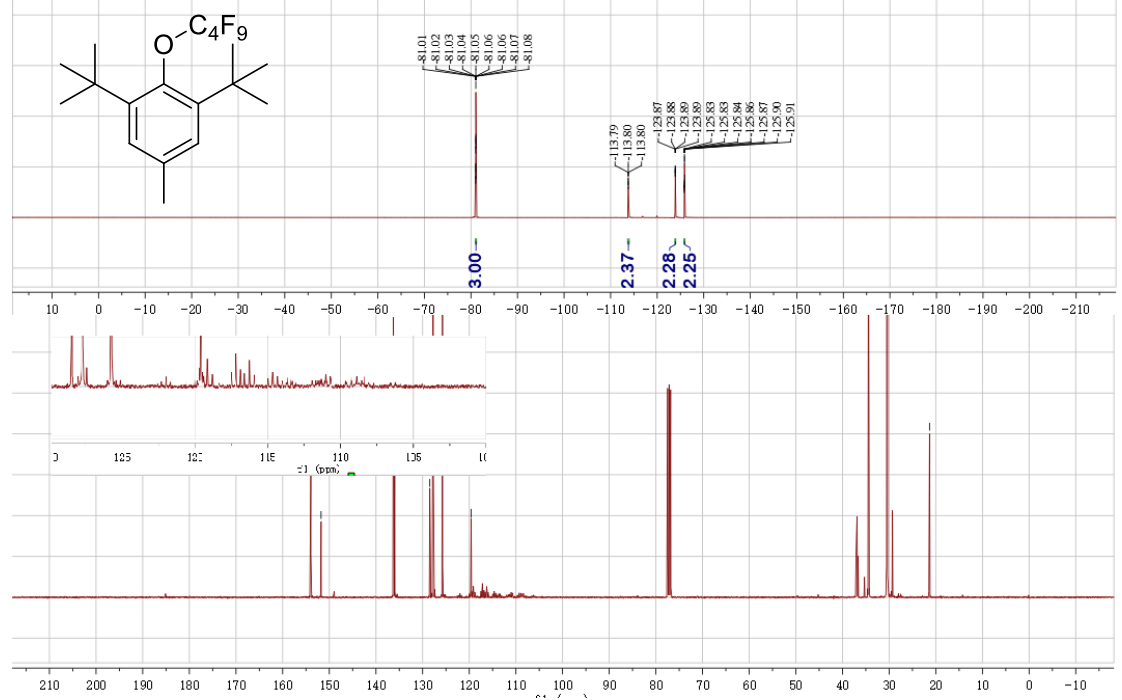


b) Radical generated by $\operatorname{Pd}(0)$

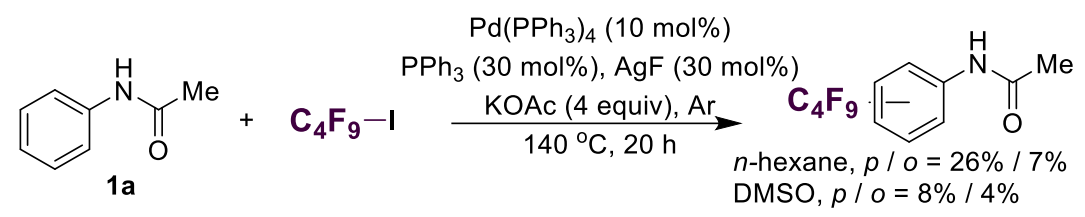

General Procedures: A mixture of $\mathbf{1 a}\left(0.2 \mathrm{mmol}, 1.0\right.$ equiv), $\mathrm{IC}_{4} \mathrm{~F}_{9}\left(130 \mu \mathrm{L}, 3\right.$ equiv), $\mathrm{Pd}\left(\mathrm{PPh}_{3}\right)_{4}$ (11.6 mg, $0.01 \mathrm{mmol}, 0.05$ equiv), KOAc (78.5 mg, $0.8 \mathrm{mmol}, 4$ equiv), $\mathrm{PPh}_{3}(15.7 \mathrm{mg}, 0.06 \mathrm{mmol}$, 0.3 equiv), $\mathrm{AgF}$ (7.6 mg, $0.06 \mathrm{mmol}, 0.3$ equiv) and $n$-hexane $(0.25 \mathrm{~mL})$ in a $15 \mathrm{~mL}$ glass vial sealed under argon atmosphere was heated at $140{ }^{\circ} \mathrm{C}$ for 20 hours. The reaction mixture cooled to room temperature and concentrated in vacuo. The resulting residue was purified by column chromatography ( $\mathrm{PE} / \mathrm{EA}=30 / 1-5 / 1)$ on silica gel to give the product. The ratio detected by ${ }^{1}$ H NMR.

When use DMSO instead of $n$-hexane $(0.25 \mathrm{~mL})$, the ratio detected isolated yield.

c) Deuteration experiment<smiles>CC(=O)Nc1ccc(C(F)(F)F)cc1</smiles>

General Procedures: A mixture of $1 \mathrm{a}\left(0.2 \mathrm{mmol}, 1.0\right.$ equiv), $\mathrm{IC}_{4} \mathrm{~F}_{9}$ ( 3 equiv), $\mathrm{Mo}(\mathrm{CO})_{6}(5.3 \mathrm{mg}$, $10 \mathrm{mmol} \%), \mathrm{Na}_{2} \mathrm{CO}_{3}(41.0 \mathrm{mg}, 2$ equiv), $\mathrm{KF}$ (23.2 mg, $30 \mathrm{mmol} \%), \mathrm{D}_{2} \mathrm{O}$ (5 equiv) and DMSO $(0.5 \mathrm{~mL})$ in a $15 \mathrm{~mL}$ glass vial sealed under $\mathrm{CO}$ atmosphere was heated at $140{ }^{\circ} \mathrm{C}$ for 48 hours. The reaction mixture cooled to room temperature and concentrated in vacuo. The resulting residue was purified by column chromatography ( $\mathrm{PE} / \mathrm{EA}=5: 1)$ on silica gel to give the product. Analyzed by ${ }^{1} \mathrm{H}$ NMR.

d) Control experiment<smiles>CC(=O)N(C(C)=O)c1ccc(N(C)C(=O)C(F)(F)F)cc1</smiles>

General Procedures: A mixture of $\mathrm{N}$-methyl- $\mathrm{N}$-phenylacetamide ( $0.2 \mathrm{mmol}, 1.0$ equiv), $\mathrm{IC}_{4} \mathrm{~F}_{9}$ (3 equiv), $\mathrm{Mo}(\mathrm{CO})_{6}(5.3 \mathrm{mg}, 10 \mathrm{mmol} \%), \mathrm{Na}_{2} \mathrm{CO}_{3}(41.0 \mathrm{mg}, 2$ equiv), $\mathrm{KF}(23.2 \mathrm{mg}, 30 \mathrm{mmol} \%)$ and DMSO $(0.5 \mathrm{~mL})$ in a $15 \mathrm{~mL}$ glass vial sealed under $\mathrm{CO}$ atmosphere was heated at $140{ }^{\circ} \mathrm{C}$ for 48 hours. Analyzed by TLC.

10. Cartesian Coordinates and Energies

$\mathrm{C}_{2} \mathrm{~F}_{5}$

\begin{tabular}{|c|c|c|c|c|c|}
\hline \multirow{2}{*}{$\begin{array}{l}\text { Center } \\
\text { Number }\end{array}$} & \multirow{2}{*}{$\begin{array}{l}\text { Atomic } \\
\text { Number }\end{array}$} & \multirow{2}{*}{$\begin{array}{c}\text { Atomic } \\
\text { Type }\end{array}$} & \multicolumn{3}{|c|}{ Coordinates (Angstroms) } \\
\hline & & & $\mathrm{X}$ & $\mathrm{Y}$ & Z \\
\hline 1 & 6 & 0 & 0.867381 & -0.000009 & -0.363982 \\
\hline 2 & 9 & 0 & 1. 509790 & -1.102608 & 0.002867 \\
\hline 3 & 9 & 0 & 1. 509736 & 1. 102626 & 0.002887 \\
\hline 4 & 6 & 0 & -0.607458 & -0.000019 & -0.019742 \\
\hline 5 & 9 & 0 & -0.815099 & 0.000023 & 1. 313902 \\
\hline 6 & 9 & 0 & -1.188864 & -1.093341 & -0.531890 \\
\hline 7 & 9 & 0 & -1.188845 & 1. 093319 & -0.531950 \\
\hline
\end{tabular}


Thermal correction to Energy=

0.030738

Thermal correction to Enthalpy=

0.031683

Thermal correction to Gibbs Free Energy=

Sum of electronic and zero-point Energies=

$-0.007323$

Sum of electronic and thermal Energies=

$-575.313683$

$-575.307463$

$-575.306519$

Sum of electronic and thermal Free Energies=

$-575.345524$

B3LYP $/ 6-311++G(d, p)-S D D / S M D / / B 3 L Y P / 6-31 G(d)-S D D \quad$ energy $=-575.53304829$

INT1

\begin{tabular}{|c|c|c|c|c|c|}
\hline \multirow{2}{*}{$\begin{array}{l}\text { Center } \\
\text { Number }\end{array}$} & \multirow{2}{*}{$\begin{array}{l}\text { Atomic } \\
\text { Number }\end{array}$} & \multirow{2}{*}{$\begin{array}{c}\text { Atomic } \\
\text { Type }\end{array}$} & \multicolumn{3}{|c|}{ Coordinates (Angstroms) } \\
\hline & & & $\mathrm{X}$ & $\mathrm{Y}$ & Z \\
\hline 1 & 6 & 0 & -3.681511 & -1.859265 & -0.463396 \\
\hline 2 & 6 & 0 & -2.439635 & -1.240453 & -0.567817 \\
\hline 3 & 6 & 0 & -2.227120 & 0.058794 & -0.058123 \\
\hline 4 & 6 & 0 & -3.307202 & 0.688133 & 0.598032 \\
\hline 5 & 6 & 0 & -4.550696 & 0.061999 & 0.696240 \\
\hline 6 & 6 & 0 & -4.752799 & -1.211616 & 0.162226 \\
\hline 7 & 1 & 0 & -5.363563 & 0.572448 & 1. 209697 \\
\hline 8 & 7 & 0 & -0.957118 & 0.615842 & -0.171947 \\
\hline 9 & 6 & 0 & -0.662805 & 1. 887387 & -0.416058 \\
\hline 10 & 8 & 0 & 0.574832 & 2. 191676 & -0.394530 \\
\hline 11 & 6 & 0 & -1.655836 & 2. 977606 & -0.778703 \\
\hline 12 & 1 & 0 & -2.599283 & 2. 576242 & -1.157945 \\
\hline 13 & 1 & 0 & -1.874475 & 3. 603276 & 0.095763 \\
\hline 14 & 1 & 0 & -1.193159 & 3. 619286 & -1.534325 \\
\hline 15 & 42 & 0 & 1. 279872 & 0.011847 & 0.027990 \\
\hline 16 & 6 & 0 & 1. 381171 & -1.919467 & 0.357026 \\
\hline 17 & 6 & 0 & 1. 221729 & 0.279224 & 2. 056359 \\
\hline 18 & 6 & 0 & 1. 453376 & -0.391169 & -1.968646 \\
\hline 19 & 6 & 0 & 3. 242029 & 0.156471 & 0.115858 \\
\hline 20 & 8 & 0 & 1. 415172 & -3.078771 & 0.551008 \\
\hline 21 & 8 & 0 & 1. 253583 & 0.363459 & 3. 217010 \\
\hline 22 & 8 & 0 & 4. 408935 & 0.279278 & 0.163217 \\
\hline 23 & 8 & 0 & 1. 615825 & -0.688568 & -3.083186 \\
\hline 24 & 1 & 0 & -3.814351 & -2.859059 & -0.871750 \\
\hline 25 & 1 & 0 & -5.721171 & -1.699049 & 0.245944 \\
\hline 26 & 1 & 0 & -3.156712 & 1. 660054 & 1. 057650 \\
\hline 27 & 1 & 0 & -1.603046 & -1.744112 & -1.043142 \\
\hline
\end{tabular}

Zero-point correction=

0. 177047 (Hartree/Particle)

Thermal correction to Energy=

Thermal correction to Enthalpy=

0.197500

0. 126270

$-961.219510$

$-961.200001$

$-961.199057$

$-961.270287$

Sum of electronic and thermal Enthalpies=

Sum of electronic and thermal Free Energies=

energy $=-961.73311586$

TS1-p

\begin{tabular}{rrrrrr} 
Center & Atomic & Atomic & \multicolumn{3}{c}{ Coordinates (Angstroms) } \\
Number & Number & Type & X & Y & $Z$ \\
--1 & 6 & 0 & -2.562006 & 0.793362 & 1.477298 \\
1 & 6 & 0 & -1.344222 & 1.243513 & 1.001582 \\
2 & 6 & 0 & -0.404951 & 0.349983 & 0.419061 \\
3 & 6 & 0 & -0.735511 & -1.032199 & 0.413047 \\
4 & 6 & 0 & -1.953320 & -1.482118 & 0.878450 \\
5 & 6 & 0 & -2.943776 & -0.567644 & 1.337762 \\
6 & 1 & 0 & -3.253857 & 1.496967 & 1.933041 \\
7 & 1 & 0 & -1.076616 & 2.287956 & 1.123026 \\
8 & 1 & 0 & -2.179607 & -2.544790 & 0.855579 \\
9 & 1 & 0 & -3.783275 & -0.945221 & 1.914582 \\
10 & 6 & 0 & -4.331313 & -0.501014 & -0.450624 \\
11 & 9 & 0 & -3.784608 & 0.315685 & -1.370819 \\
12 & & 0 & & &
\end{tabular}




\begin{tabular}{|c|c|c|c|c|c|}
\hline 13 & 9 & 0 & -4.417723 & -1.756131 & -0.945189 \\
\hline 14 & 6 & 0 & -5.700401 & -0.006612 & -0.046650 \\
\hline 15 & 9 & 0 & -6.589795 & 0.042144 & -1.070590 \\
\hline 16 & 9 & 0 & -5.617020 & 1. 235297 & 0.468632 \\
\hline 17 & 9 & 0 & -6.223440 & -0.820046 & 0.895150 \\
\hline 18 & 7 & 0 & 0.832183 & 0.723683 & -0.056602 \\
\hline 19 & 6 & 0 & 1. 158951 & 1. 878912 & -0.643588 \\
\hline 20 & 8 & 0 & 2. 395518 & 2. 050238 & -0.875347 \\
\hline 21 & 6 & 0 & 0.184728 & 2. 937774 & -1.124453 \\
\hline 22 & 1 & 0 & 0.128019 & 3. 764254 & -0.405043 \\
\hline 23 & 1 & 0 & 0.568119 & 3. 346012 & -2.063928 \\
\hline 24 & 1 & 0 & -0.823046 & 2.542101 & -1.274983 \\
\hline 25 & 42 & 0 & 3. 029443 & -0.047039 & -0.068077 \\
\hline 26 & 6 & 0 & 4. 985776 & -0.075298 & -0.298634 \\
\hline 27 & 6 & 0 & 2. 845958 & -0.882723 & -1.927436 \\
\hline 28 & 6 & 0 & 3. 310303 & 0.656366 & 1. 832748 \\
\hline 29 & 6 & 0 & 3. 073081 & -1.863234 & 0.671248 \\
\hline 30 & 8 & 0 & 6. 149236 & -0.057802 & -0.448012 \\
\hline 31 & 8 & 0 & 2. 801256 & -1.424904 & -2.956201 \\
\hline 32 & 8 & 0 & 3. 072430 & -2.951336 & 1. 115210 \\
\hline 33 & 8 & 0 & 3. 524137 & 0.988983 & 2. 927035 \\
\hline 34 & 1 & 0 & 0.011250 & -1.725797 & 0.038178 \\
\hline
\end{tabular}

Zero-point correction=

Thermal correction to Energy=

0.201592 (Hartree/Particle)

Thermal correction to Enthalpy=

Thermal correction to Gibbs Free Energy=

Sum of electronic and zero-point Energies=

Sum of electronic and thermal Energies=

Sum of electronic and thermal Enthalpies=

Sum of electronic and thermal Free Energies=

0.228512

0.229456

0. 137773

$-1536.529693$

$-1536.502774$

$-1536.501830$

$-1536.593513$

B3LYP/6-311++G (d, p) -SDD/SMD//B3LYP/6-31G (d) -SDD energy $=-1537.26289062$

TS1-o

\begin{tabular}{|c|c|c|c|c|c|}
\hline \multirow{2}{*}{$\begin{array}{l}\text { Center } \\
\text { Number }\end{array}$} & \multirow{2}{*}{$\begin{array}{l}\text { Atomic } \\
\text { Number }\end{array}$} & \multirow{2}{*}{$\begin{array}{c}\text { Atomic } \\
\text { Type }\end{array}$} & \multicolumn{3}{|c|}{ Coordinates (Angstroms) } \\
\hline & & & $X$ & $\mathrm{Y}$ & Z \\
\hline 1 & 6 & 0 & 2. 645579 & 3. 310878 & -0.604946 \\
\hline 2 & 6 & 0 & 1. 515964 & 2. 906272 & 0.106343 \\
\hline 3 & 6 & 0 & 0.947644 & 1. 631238 & -0.092170 \\
\hline 4 & 6 & 0 & 1. 609915 & 0.744368 & -1.009947 \\
\hline 5 & 6 & 0 & 2. 687517 & 1. 219230 & -1.798916 \\
\hline 6 & 6 & 0 & 3. 232544 & 2. 472019 & -1.566933 \\
\hline 7 & 1 & 0 & 3. 048745 & 4. 307305 & -0.437286 \\
\hline 8 & 1 & 0 & 1. 031339 & 3. 608622 & 0.775732 \\
\hline 9 & 1 & 0 & 3. 113375 & 0.561364 & -2.551455 \\
\hline 10 & 1 & 0 & 4. 085559 & 2. 818189 & -2.145269 \\
\hline 11 & 6 & 0 & 2. 700023 & -0.555706 & 0.483023 \\
\hline 12 & 9 & 0 & 3. 914821 & -0.005586 & 0.686082 \\
\hline 13 & 9 & 0 & 2. 018052 & -0.578662 & 1. 644392 \\
\hline 14 & 6 & 0 & 2.844393 & -1.949055 & -0.090036 \\
\hline 15 & 9 & 0 & 3. 540414 & -2.785195 & 0.721509 \\
\hline 16 & 9 & 0 & 3.507513 & -1.891656 & -1.264525 \\
\hline 17 & 9 & 0 & 1. 641858 & -2.498579 & -0.306918 \\
\hline 18 & 7 & 0 & -0.211622 & 1. 176849 & 0.495257 \\
\hline 19 & 6 & 0 & -0.688374 & 1. 494325 & 1. 702812 \\
\hline 20 & 8 & 0 & -1.852590 & 1. 063834 & 1. 968973 \\
\hline 21 & 6 & 0 & 0.070756 & 2. 232554 & 2. 788592 \\
\hline 22 & 1 & 0 & -0.251108 & 1. 832647 & 3. 753968 \\
\hline 23 & 1 & 0 & 1. 152580 & 2. 118163 & 2. 681162 \\
\hline 24 & 1 & 0 & -0.168915 & 3. 303187 & 2. 775283 \\
\hline 25 & 42 & 0 & -2.071272 & -0.125839 & -0.026764 \\
\hline 26 & 6 & 0 & -3.867523 & -0.926717 & 0.069151 \\
\hline 27 & 6 & 0 & -1.360772 & -1.813977 & 0.895357 \\
\hline 28 & 6 & 0 & -2.900309 & 1. 446878 & -1.035264 \\
\hline 29 & 6 & 0 & -1.754910 & -0.990376 & -1.756996 \\
\hline 30 & 8 & 0 & -4.946166 & -1.381537 & 0.152255 \\
\hline 31 & 8 & 0 & -1.038164 & -2.826020 & 1. 368079 \\
\hline
\end{tabular}




\begin{tabular}{rrrrrr}
32 & 8 & 0 & -1.531447 & -1.497049 & -2.794142 \\
33 & 8 & 0 & -3.416809 & 2.281746 & -1.660828 \\
34 & 1 & 0 & 1.046020 & -0.124469 & -1.334835 \\
\hline
\end{tabular}

Zero-point correction=

Thermal correction to Energy=

Thermal correction to Enthalpy=

Thermal correction to Gibbs Free Energy=

Sum of electronic and zero-point Energies=

Sum of electronic and thermal Energies=

Sum of electronic and thermal Enthalpies=

Sum of electronic and thermal Free Energies=

B3LYP/6-311++G (d, p)-SDD/SMD//B3LYP/6-31G(d)-SDD energy= -1537. 26233206
0.201603 (Hartree/Particle)

0.228406

0.229351

0.139390

$-1536.530515$

$-1536.503711$

$-1536.502767$

$-1536.592727$

TS1-m

\begin{tabular}{|c|c|c|c|c|c|}
\hline \multirow{2}{*}{$\begin{array}{l}\text { Center } \\
\text { Number }\end{array}$} & \multirow{2}{*}{$\begin{array}{l}\text { Atomic } \\
\text { Number }\end{array}$} & \multirow{2}{*}{$\begin{array}{c}\text { Atomic } \\
\text { Type }\end{array}$} & \multicolumn{3}{|c|}{ Coordinates (Angstroms) } \\
\hline & & & $X$ & Y & Z \\
\hline 1 & 6 & 0 & -1.724046 & 2. 409746 & 0.264520 \\
\hline 2 & 6 & 0 & -0.604803 & 1. 588174 & 0.418077 \\
\hline 3 & 6 & 0 & -0.510972 & 0.338549 & -0.250070 \\
\hline 4 & 6 & 0 & -1.562380 & -0.026843 & -1.101734 \\
\hline 5 & 6 & 0 & -2.753050 & 0.746972 & -1.172128 \\
\hline 6 & 6 & 0 & -2.788924 & 2. 022932 & -0.539044 \\
\hline 7 & 1 & 0 & -1.750103 & 3. 370869 & 0.772938 \\
\hline 8 & 1 & 0 & 0.231343 & 1. 893495 & 1. 039516 \\
\hline 9 & 1 & 0 & -3.436015 & 0.557391 & -1.997716 \\
\hline 10 & 1 & 0 & -3.657454 & 2. 663091 & -0.660763 \\
\hline 11 & 6 & 0 & -4.065131 & -0.480744 & 0.072121 \\
\hline 12 & 9 & 0 & -3.518165 & -0.576657 & 1. 294573 \\
\hline 13 & 9 & 0 & -4.055046 & -1.691655 & -0.527932 \\
\hline 14 & 6 & 0 & -5.484290 & 0.041911 & 0.150629 \\
\hline 15 & 9 & 0 & -6.329135 & -0.809909 & 0.781224 \\
\hline 16 & 9 & 0 & -5.522801 & 1. 211908 & 0.809447 \\
\hline 17 & 9 & 0 & -5.968633 & 0.236707 & -1.093720 \\
\hline 18 & 7 & 0 & 0.650876 & -0.398392 & -0.087082 \\
\hline 19 & 6 & 0 & 0.749585 & -1.724246 & -0.018057 \\
\hline 20 & 8 & 0 & 1. 932075 & -2.191035 & 0.029241 \\
\hline 21 & 6 & 0 & -0.409810 & -2.699017 & 0.080840 \\
\hline 22 & 1 & 0 & -0.598741 & -3.167463 & -0.893155 \\
\hline 23 & 1 & 0 & -1.335733 & -2.228397 & 0.419898 \\
\hline 24 & 1 & 0 & -0.121649 & -3.493827 & 0.775100 \\
\hline 25 & 42 & 0 & 2. 949697 & -0.088796 & 0.025427 \\
\hline 26 & 6 & 0 & 4. 879367 & -0.482216 & 0.094104 \\
\hline 27 & 6 & 0 & 3. 086055 & -0.045118 & -2.015981 \\
\hline 28 & 6 & 0 & 2. 951030 & -0.015149 & 2. 069506 \\
\hline 29 & 6 & 0 & 3. 316924 & 1.837401 & 0.022551 \\
\hline 30 & 8 & 0 & 6. 021025 & -0.751357 & 0.133110 \\
\hline 31 & 8 & 0 & 3. 235466 & 0.037637 & -3.167319 \\
\hline 32 & 8 & 0 & 3.506981 & 2. 997780 & 0.022048 \\
\hline 33 & 8 & 0 & 3. 026956 & 0.088209 & 3. 226921 \\
\hline 34 & 1 & 0 & -1.488376 & -0.934502 & -1.690531 \\
\hline
\end{tabular}

Zero-point correction=

Thermal correction to Energy=

0.201413 (Hartree/Particle)

Thermal correction to Enthalpy=

0.228282

0.229226

0. 137595

$-1536.526211$

$-1536.499341$

$-1536.498397$

$-1536.590028$

Sum of electronic and thermal Energies=

Sum of electronic and thermal Enthalpies=

Sum of electronic and thermal Free Energies=

energy $=-1537.25830992$

INT2-p

\begin{tabular}{lllll} 
Center & Atomic & Atomic & \multicolumn{3}{c}{ Coordinates (Angstroms) } \\
Number & Number & Type & $X$ & $Y$
\end{tabular}




\begin{tabular}{|c|c|c|c|c|c|}
\hline 1 & 6 & 0 & -2.689524 & 0.861550 & 1. 052631 \\
\hline 2 & 6 & 0 & -1.430835 & 1. 288509 & 0.748582 \\
\hline 3 & 6 & 0 & -0.424953 & 0.422364 & 0. 209329 \\
\hline 4 & 6 & 0 & -0.762410 & -0.971069 & 0.120307 \\
\hline 5 & 6 & 0 & -2.001990 & -1.449589 & 0.395506 \\
\hline 6 & 6 & 0 & -3.149801 & -0.552617 & 0.799419 \\
\hline 7 & 1 & 0 & -3.391165 & 1. 547411 & 1. 517646 \\
\hline 8 & 1 & 0 & -1.162329 & 2. 313501 & 0.985616 \\
\hline 9 & 1 & 0 & -2.209712 & -2.512083 & 0.314477 \\
\hline 10 & 1 & 0 & -3.634383 & -0.956828 & 1. 706437 \\
\hline 11 & 6 & 0 & -4.268466 & -0.641100 & -0.268603 \\
\hline 12 & 9 & 0 & -3.852294 & -0.165484 & -1.472236 \\
\hline 13 & 9 & 0 & -4.626124 & -1.955494 & -0.441228 \\
\hline 14 & 6 & 0 & -5.588141 & 0.090550 & 0.058983 \\
\hline 15 & 9 & 0 & -6.542514 & -0.226828 & -0.833233 \\
\hline 16 & 9 & 0 & -5.435143 & 1. 425909 & 0. 038509 \\
\hline 17 & 9 & 0 & -6.032308 & -0.260730 & 1. 283665 \\
\hline 18 & 7 & 0 & 0.855093 & 0.798229 & -0.113169 \\
\hline 19 & 6 & 0 & 1. 276971 & 1.996752 & -0.531632 \\
\hline 20 & 8 & 0 & 2. 538053 & 2. 132216 & -0.637353 \\
\hline 21 & 6 & 0 & 0. 393559 & 3. 153819 & -0.955303 \\
\hline 22 & 1 & 0 & 0.251714 & 3. 862300 & -0.129113 \\
\hline 23 & 1 & 0 & 0.901278 & 3. 687614 & -1.763661 \\
\hline 24 & 1 & 0 & -0.591797 & 2. 820157 & -1.292623 \\
\hline 25 & 42 & 0 & 3. 021542 & -0.058626 & -0.019212 \\
\hline 26 & 6 & 0 & 4. 989244 & -0.139594 & -0.078523 \\
\hline 27 & 6 & 0 & 2. 981800 & -0.674868 & -1.970832 \\
\hline 28 & 6 & 0 & 3. 146687 & 0.413019 & 1. 967847 \\
\hline 29 & 6 & 0 & 2. 944214 & -1.946601 & 0.507349 \\
\hline 30 & 8 & 0 & 6. 161635 & -0.151944 & -0.121725 \\
\hline 31 & 8 & 0 & 3. 012780 & -1.097792 & -3.054626 \\
\hline 32 & 8 & 0 & 2. 871209 & -3.077347 & 0.820250 \\
\hline 33 & 8 & 0 & 3. 267592 & 0.607476 & 3. 108833 \\
\hline 34 & 1 & 0 & 0.033127 & -1.651353 & -0.172351 \\
\hline
\end{tabular}

Zero-point correction=

0.203943 (Hartree/Particle)

Thermal correction to Energy=

0.230342

Thermal correction to Enthalpy=

0. 231287

0. 142017

$-1536.559413$

$\begin{array}{ll}\text { Sum of electronic and thermal Energies= } & -1536.533013 \\ \text { Sum of electronic and thermal Enthalpies= } & -1536.532069 \\ \text { Sum of electronic and thermal Free Energies= } & -1536.621339\end{array}$

$\begin{array}{ll}\text { Sum of electronic and thermal Energies= } & -1536.533013 \\ \text { Sum of electronic and thermal Enthalpies= } & -1536.532069 \\ \text { Sum of electronic and thermal Free Energies= } & -1536.621339\end{array}$

$\begin{array}{ll}\text { Sum of electronic and thermal Energies= } & -1536.533013 \\ \text { Sum of electronic and thermal Enthalpies= } & -1536.532069 \\ \text { Sum of electronic and thermal Free Energies= } & -1536.621339\end{array}$

B3LYP/6-311++G (d, p)-SDD/SMD//B3LYP/6-31G (d) -SDD energy $=-1537.29159451$

INT2-o

\begin{tabular}{rrrrrr} 
Center & Atomic & Atomic & \multicolumn{3}{c}{ Coordinates (Angstroms) } \\
Number & Number & Type & X & Y & $Z$ \\
-1 & 6 & 0 & 2.609645 & 3.183319 & -0.809571 \\
2 & 6 & 0 & 1.477613 & 2.853276 & -0.045726 \\
3 & 6 & 0 & 0.971195 & 1.558626 & 0.003831 \\
4 & 6 & 0 & 1.758942 & 0.425788 & -0.651713 \\
5 & 6 & 0 & 2.777430 & 0.913498 & -1.654353 \\
6 & 6 & 0 & 3.203930 & 2.207706 & -1.656272 \\
7 & 1 & 0 & 2.958703 & 4.211767 & -0.837693 \\
8 & 1 & 0 & 0.920167 & 3.652261 & 0.433726 \\
9 & 1 & 0 & 3.192943 & 0.190056 & -2.347575 \\
10 & 1 & 0 & 3.977749 & 2.516077 & -2.357124 \\
11 & 6 & 0 & 2.454877 & -0.423364 & 0.452197 \\
12 & 9 & 0 & 3.498080 & 0.258891 & 1.011128 \\
13 & 9 & 0 & 1.591704 & -0.729828 & 1.462582 \\
14 & 6 & 0 & 3.019791 & -1.784225 & -0.015392 \\
15 & 9 & 0 & 3.504548 & -2.475127 & 1.031221 \\
16 & 9 & 0 & 4.025522 & -1.630648 & -0.900749 \\
17 & 9 & 0 & 2.059700 & -2.519318 & -0.598571 \\
18 & 7 & 0 & -0.238396 & 1.185808 & 0.529247 \\
19 & 6 & 0 & -0.880620 & 1.716194 & 1.575110 \\
& 9 & & & &
\end{tabular}




\begin{tabular}{|c|c|c|c|c|c|}
\hline 20 & 8 & 0 & -2.075534 & 1. 320712 & 1. 751693 \\
\hline 21 & 6 & 0 & -0.273842 & 2. 668824 & 2. 584424 \\
\hline 22 & 1 & 0 & -0.689678 & 2. 433106 & 3. 568210 \\
\hline 23 & 1 & 0 & 0.816894 & 2. 597303 & 2. 611891 \\
\hline 24 & 1 & 0 & -0.545218 & 3. 706084 & 2. 349807 \\
\hline 25 & 42 & 0 & -2.055167 & -0.161999 & -0.048950 \\
\hline 26 & 6 & 0 & -3.870831 & -0.922750 & -0.074369 \\
\hline 27 & 6 & 0 & -1.494645 & -1.710754 & 1. 171541 \\
\hline 28 & 6 & 0 & -2.698588 & 1. 261751 & -1.367675 \\
\hline 29 & 6 & 0 & -1.554412 & -1.267034 & -1.588107 \\
\hline 30 & 8 & 0 & -4.963061 & -1.351436 & -0.067176 \\
\hline 31 & 8 & 0 & -1.236329 & -2.652640 & 1.802358 \\
\hline 32 & 8 & 0 & -1.224758 & -1.916840 & -2.511234 \\
\hline 33 & 8 & 0 & -3.100470 & 2. 004347 & -2.168716 \\
\hline 34 & 1 & 0 & 1.036555 & -0.257343 & -1.119500 \\
\hline \multicolumn{4}{|c|}{ Zero-point correction $=$} & \multicolumn{2}{|c|}{ 0.203591 (Hartree/Particle) } \\
\hline \multicolumn{4}{|c|}{ Thermal correction to Energy= } & \multicolumn{2}{|c|}{0.230050} \\
\hline \multicolumn{4}{|c|}{ Thermal correction to Enthalpy= } & \multicolumn{2}{|l|}{0.230994} \\
\hline \multicolumn{4}{|c|}{ Thermal correction to Gibbs Free Energy= } & \multicolumn{2}{|l|}{0.142075} \\
\hline \multicolumn{4}{|c|}{ Sum of electronic and zero-point Energies= } & \multicolumn{2}{|c|}{-1536.559402} \\
\hline \multicolumn{4}{|c|}{ Sum of electronic and thermal Energies= } & \multicolumn{2}{|c|}{-1536.532943} \\
\hline \multicolumn{4}{|c|}{ Sum of electronic and thermal Enthalpies= } & \multicolumn{2}{|c|}{-1536.531999} \\
\hline \multirow{2}{*}{\multicolumn{4}{|c|}{$\begin{array}{l}\text { Sum of electronic and thermal Free Energies }= \\
\text { B3LYP } / 6-311++G(d, p)-S D D / S M D / / B 3 L Y P / 6-31 G(d)-S D\end{array}$}} & \multicolumn{2}{|c|}{-1536.620918} \\
\hline & & & & nergy & \\
\hline
\end{tabular}

INT2-m

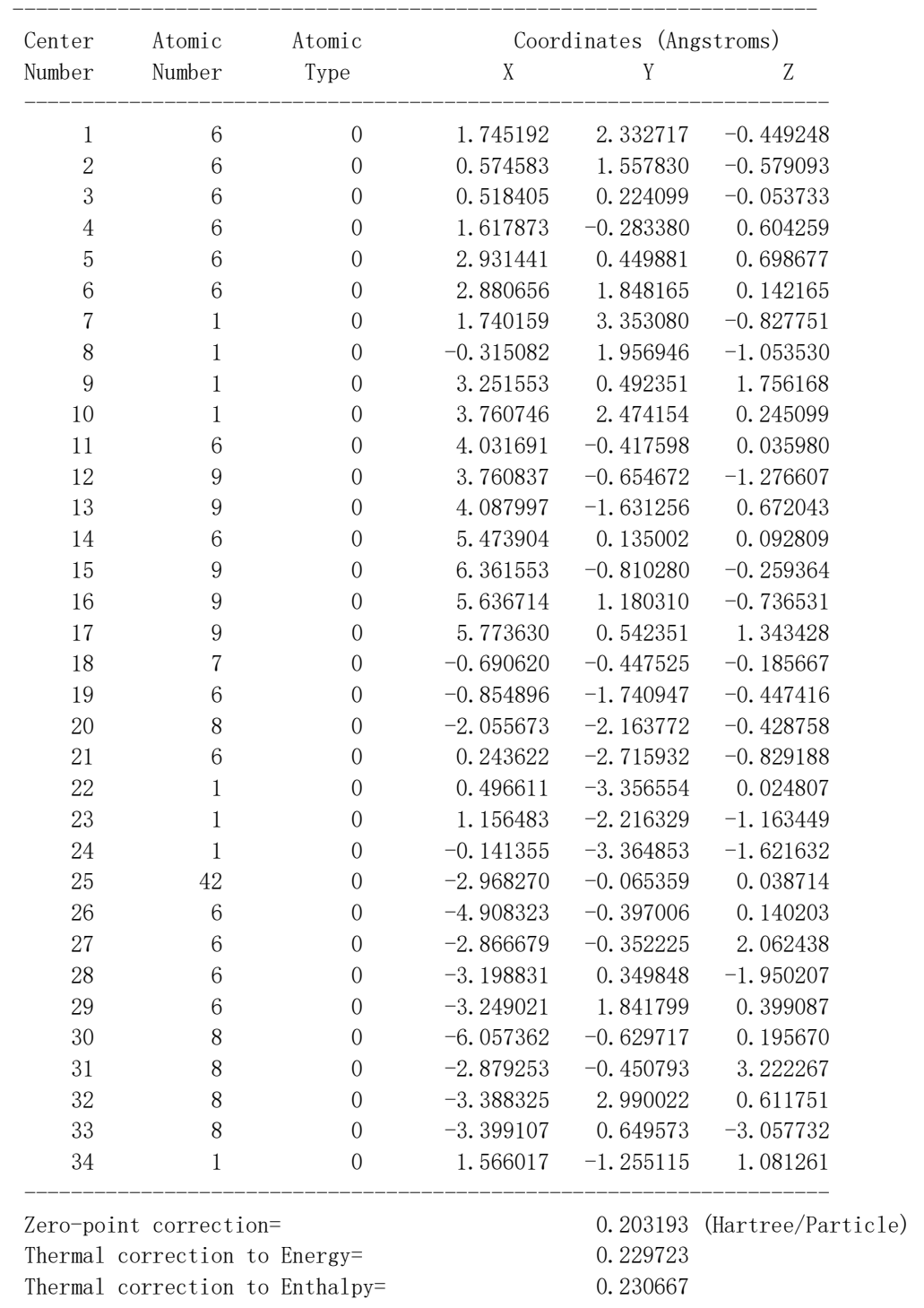


Thermal correction to Gibbs Free Energy= Sum of electronic and zero-point Energies= Sum of electronic and thermal Energies= Sum of electronic and thermal Enthalpies= Sum of electronic and thermal Free Energies= B3LYP/6-311++G (d, p) -SDD/SMD//B3LYP/6-31G (d) -SDD

\subsection{5}

$-1536.553352$

$-1536.526822$

$-1536.525878$

$-1536.615360$ 


\section{References}

[1](a) Lee, C.; Yang, W.; Parr, R. G. Development of the Colle-Salvetti correlation-energy formula into a functional of the electron density. Phys. Rev. B 1988, 37, 785-789. (b) Stephens, P. J.; Devlin, F. J.; Chabalowski, C. F.; Frisch, M. J. Ab Initio Calculation of Vibrational Absorption and Circular Dichroism Spectra Using Density Functional Force Fields. J. Phys. Chem. 1994, 98, 11623-11627.

[2] (a) Dolg, M.; Wedig, U.; Stoll, H. and Preuss, H. Energy-adjusted ab initio pseudopotentials for the first row transition elements. J. Chem. Phys. 1987, 86, 866-872. (b) Andrae, D.; Häußermann, U.; Dolg, M.; Stoll, H. and Preuß, H. Energy-adjusted $a b$ initio pseudopotentials for the second and third row transition elements. Theor. Chim. Acta. 1990, 77, 123-141.

[3] Hariharan, P. C.; Pople, J. A. The Influence of Polarization Functions on Molecular Orbital Hydrogenation Energies. Theoret. Chim. Acta. 1973, 28, 213-222.

[4] (a) Fukui, K. A Formulation of the Reaction Coordinate. J. Phys. Chem. 1970, 74, 4161-4163.

(b) Fukui, K. The Path of Chemical Reactions-The IRC Approach. Acc. Chem. Res. 1981, 14, 363-368.

[5] Marenich, A. V.; Cramer, C. J.; Truhlar, D. G. Universal Solvation Model Based on Solute Electron Density and on a Continuum Model of the Solvent Defined by the Bulk Dielectric Constant and Atomic Surface Tensions. J. Phys. Chem. B 2009, 113, 6378-6396.

[6] Frisch, M. J.; Trucks, G. W.; Schlegel, H. B.; Scuseria, G. E.; Robb, M. A.; Cheeseman, J. R.; Scalmani, G.; Barone, V.; Mennucci, B.; Petersson, G. A.; Nakatsuji, H.; Caricato, M.; Li, X.; Hratchian, H. P.; Izmaylov, A. F.; Bloino, J.; Zheng, G.; Sonnenberg, J. L.; Hada, M.; Ehara, M.; Toyota, K.; Fukuda, R.; Hasegawa, J.; Ishida, M.; Nakajima, T.; Honda, Y.; Kitao, O.; Nakai, H.; Vreven, T.; Montgomery, J. A.; Peralta, J. E.; Ogliaro, F.; Bearpark, M.; Heyd, J. J.; Brothers, E.; Kudin, K. N.; Staroverov, V. N.; Kobayashi, R.; Normand, J.; Raghavachari, K.; Rendell, A.; Burant, J. C.; Iyengar, S. S.; Tomasi, J.; Cossi, M.; Rega, N.; Millam, N. J.; Klene, M.; Knox, J. E.; Cross, J. B.; Bakken, V.; Adamo, C.; Jaramillo, J.; Gomperts, R.; Stratmann, R. E.; Yazyev, O.; Austin, A. J.; Cammi, R.; Pomelli, C.; Ochterski, J. W.; Martin, R. L.; Morokuma, K.; Zakrzewski, V. G.; Voth, G. A.; Salvador, P.; Dannenberg, J. J.; Dapprich, S.; Daniels, A. D.; Farkas, O.; Foresman, J. B.; Ortiz, J. V.; Cioslowski, J.; Fox, D. J.; Gaussian 09, Revision C.01; Gaussian, Inc.: Wallingford, CT, 2010.

[7] Legault, C. Y. CYLview, 1.0b; Université de Sherbrooke: Sherbrooke, Quebec, Canada, 2009. http://www.Cylview.org 
NMR Spectra (Perfluorobenzene used as a co-solvent helps compounds dissolve in d6-DMSO) ${ }^{1} \mathrm{H}$ NMR Spectra of $\mathbf{3 a}$

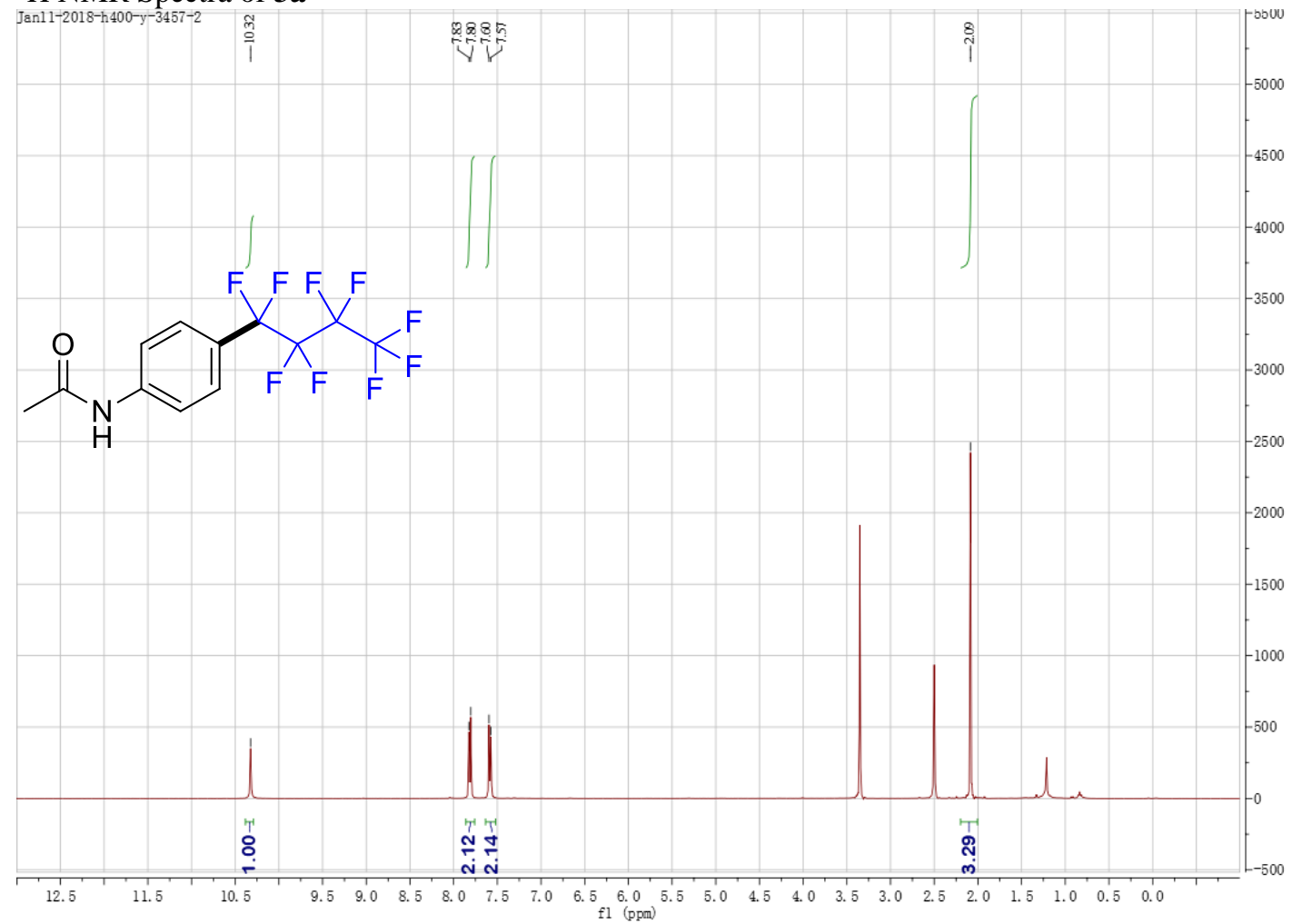

${ }^{19}$ F NMR Spectra of 3a

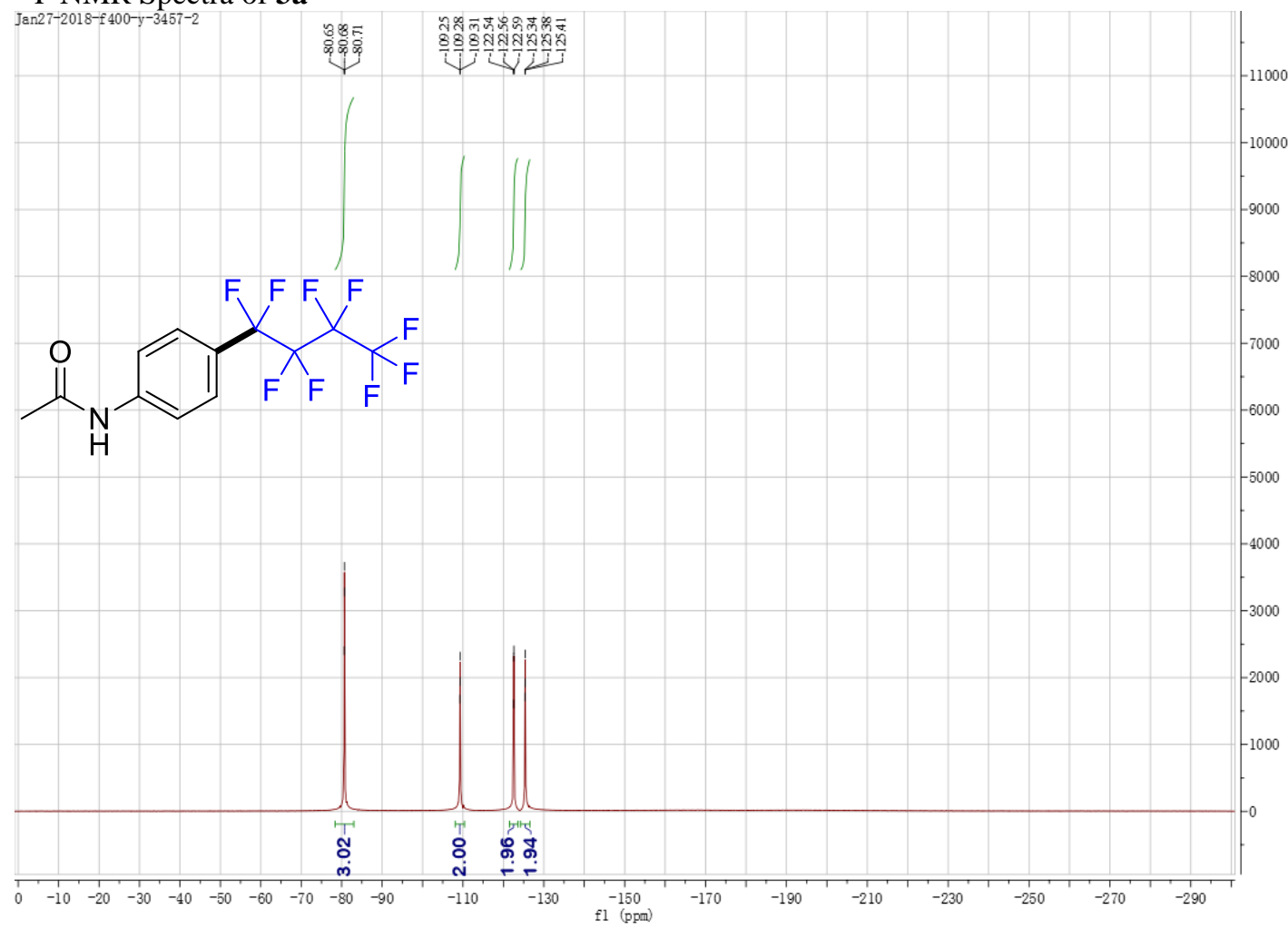


${ }^{13}$ C NMR Spectra of $\mathbf{3 a}$

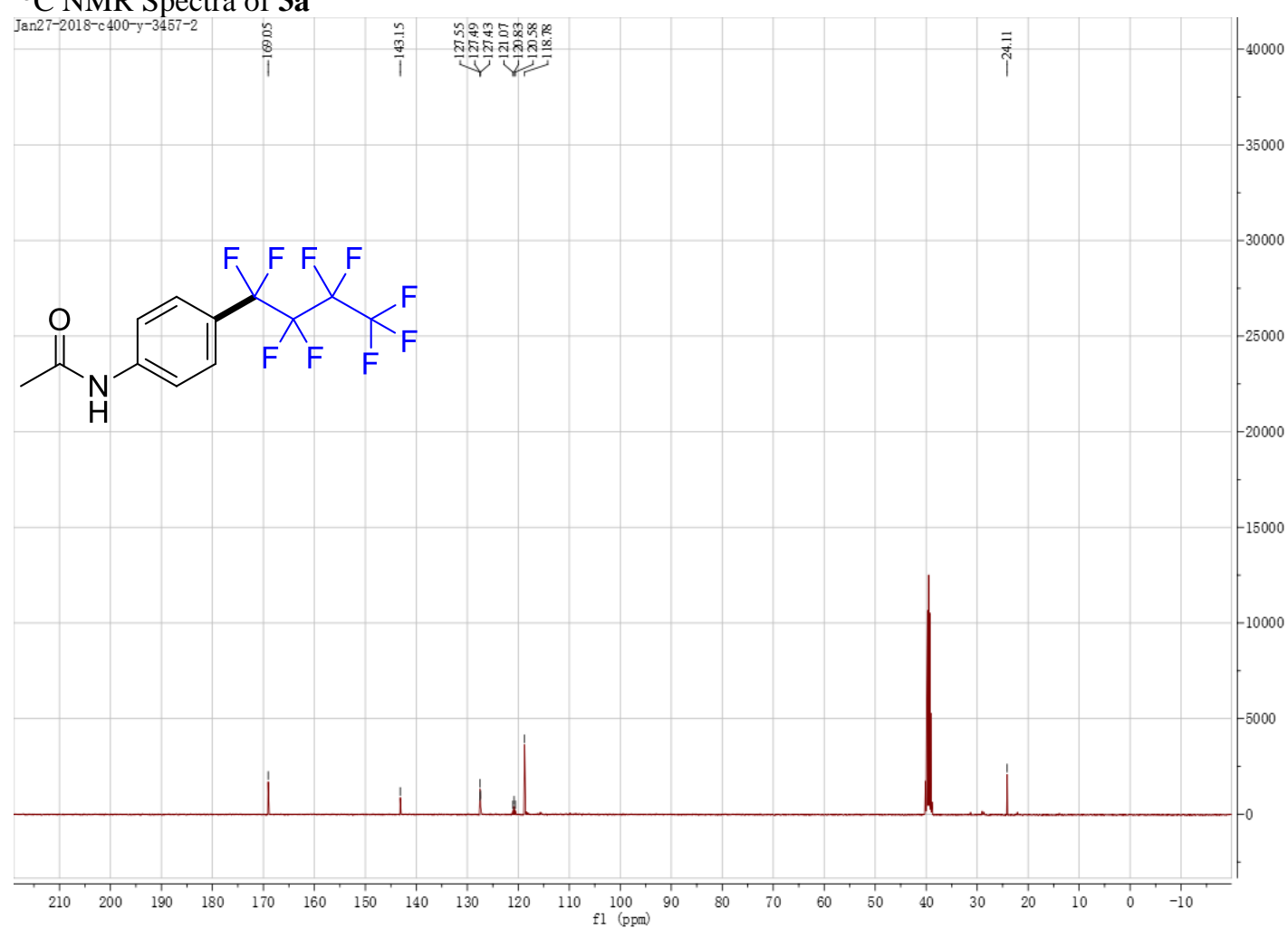

${ }^{1} \mathrm{H}$ NMR Spectra of $\mathbf{3 b}$

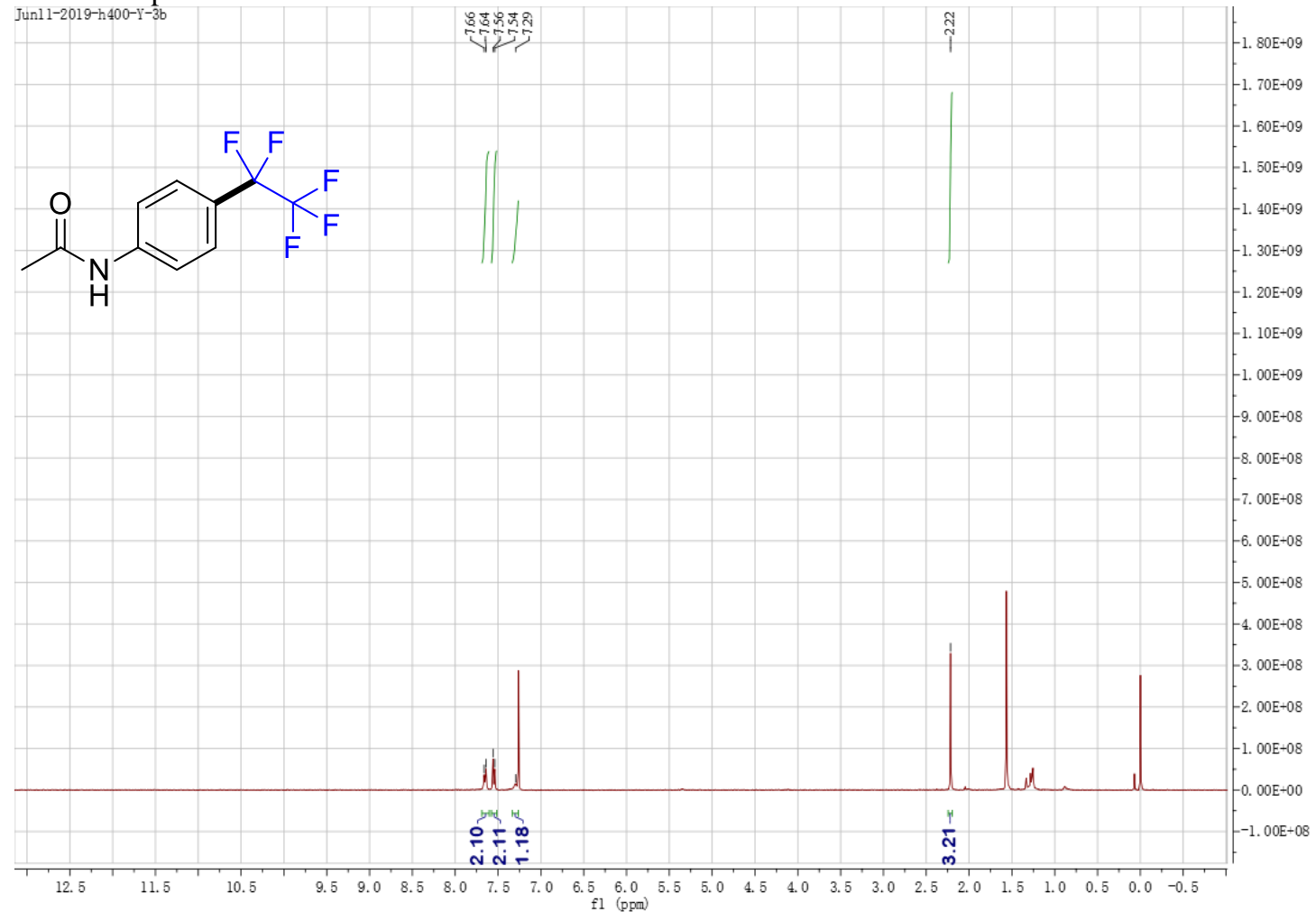


${ }^{19} \mathrm{~F}$ NMR Spectra of $\mathbf{3 b}$

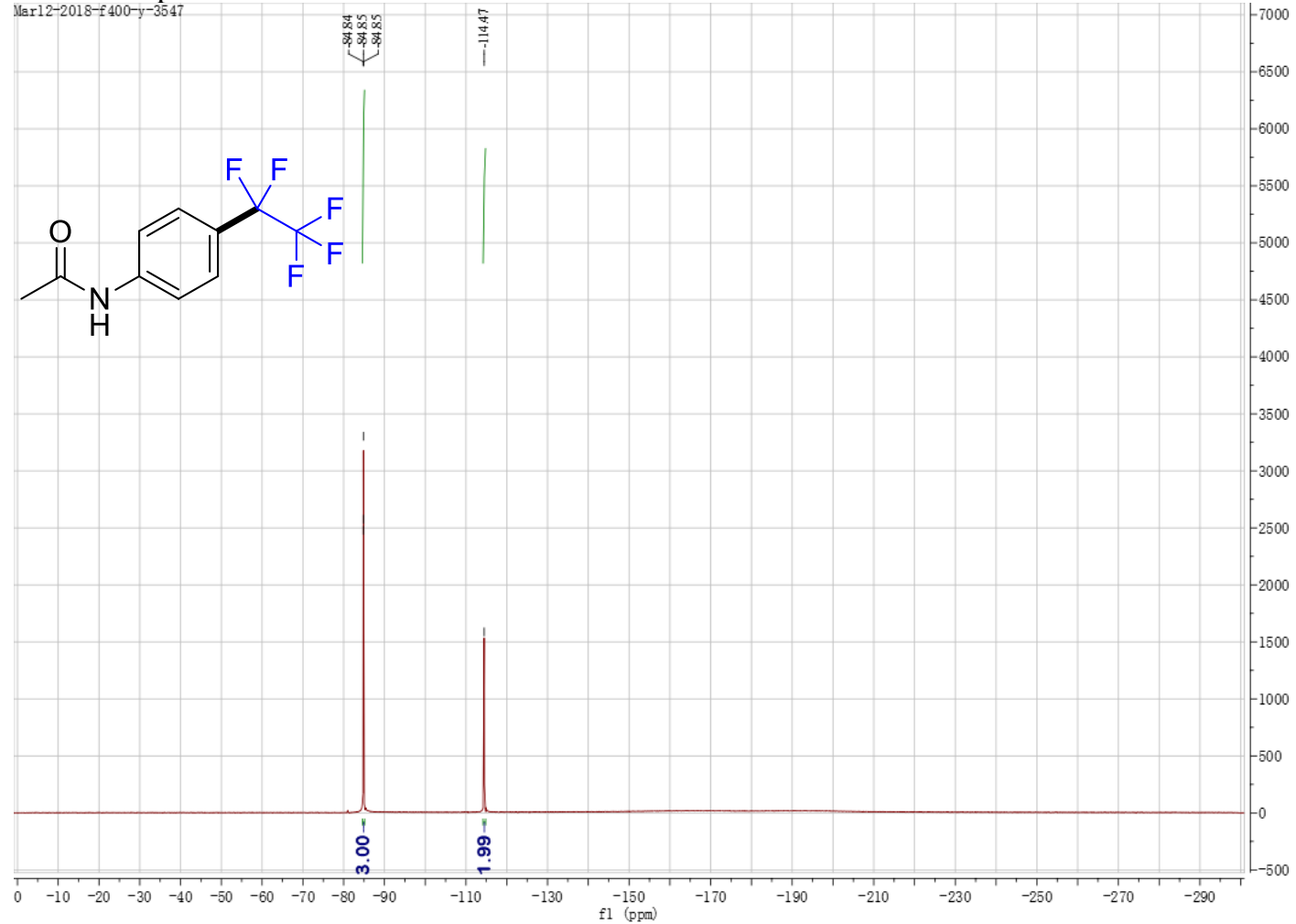

${ }^{13} \mathrm{C}$ NMR Spectra of $\mathbf{3 b}$

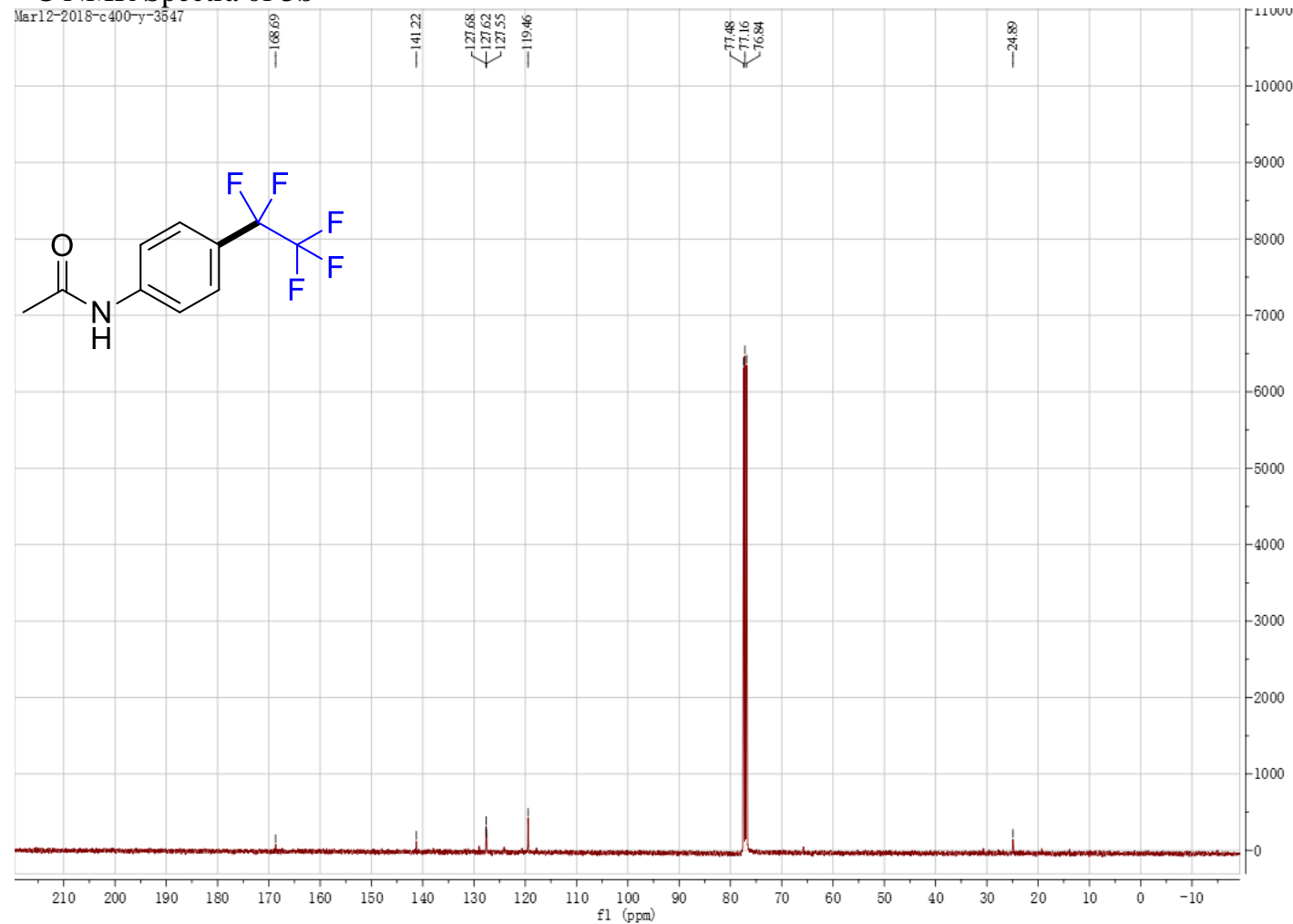


${ }^{1} \mathrm{H}$ NMR Spectra of $\mathbf{3 c}$

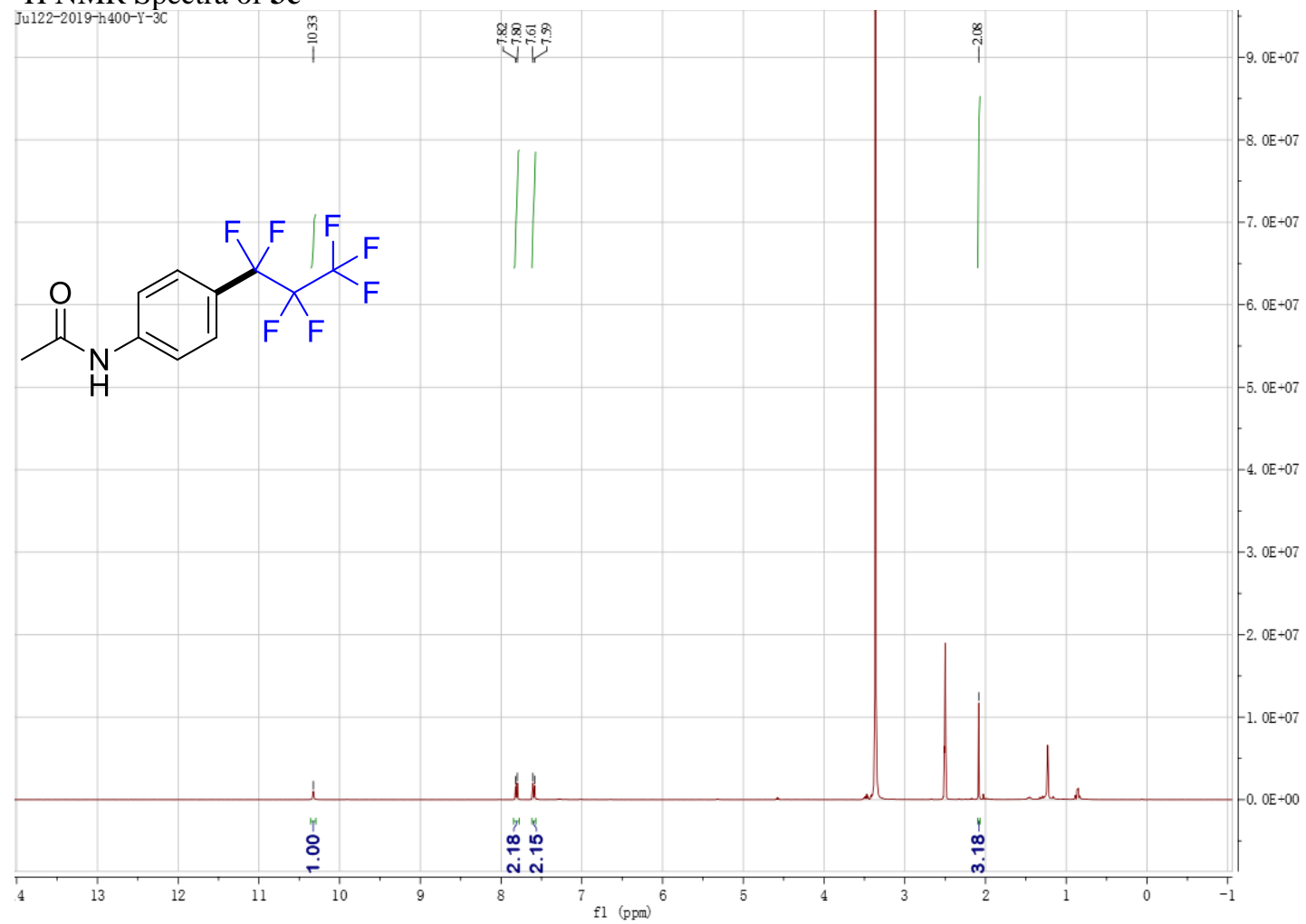

${ }^{19} \mathrm{~F}$ NMR Spectra of $\mathbf{3 c}$

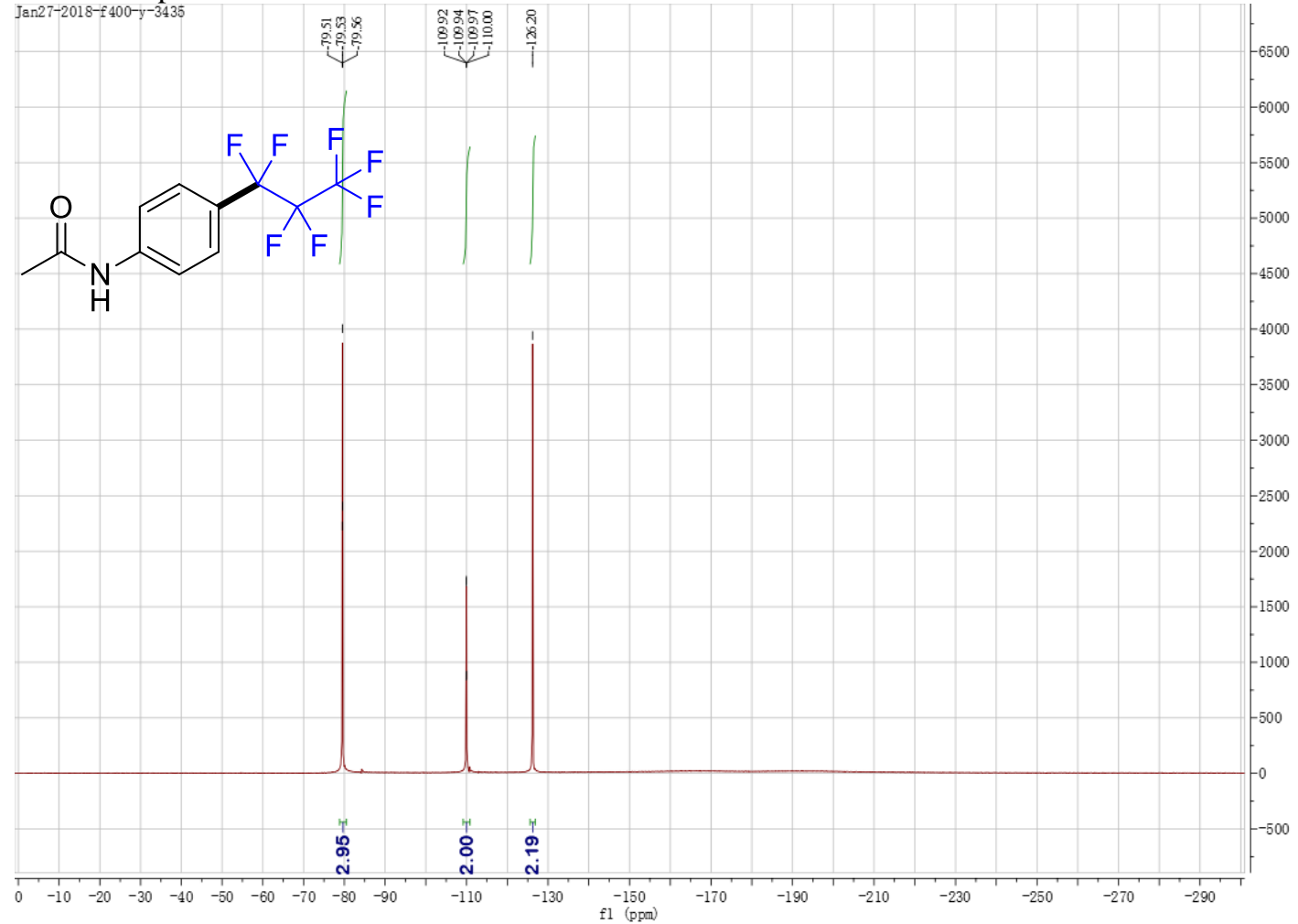


${ }_{\text {Jan } 27-2018-c 400-y-3435}^{13}$ NMR Spectra of 3c

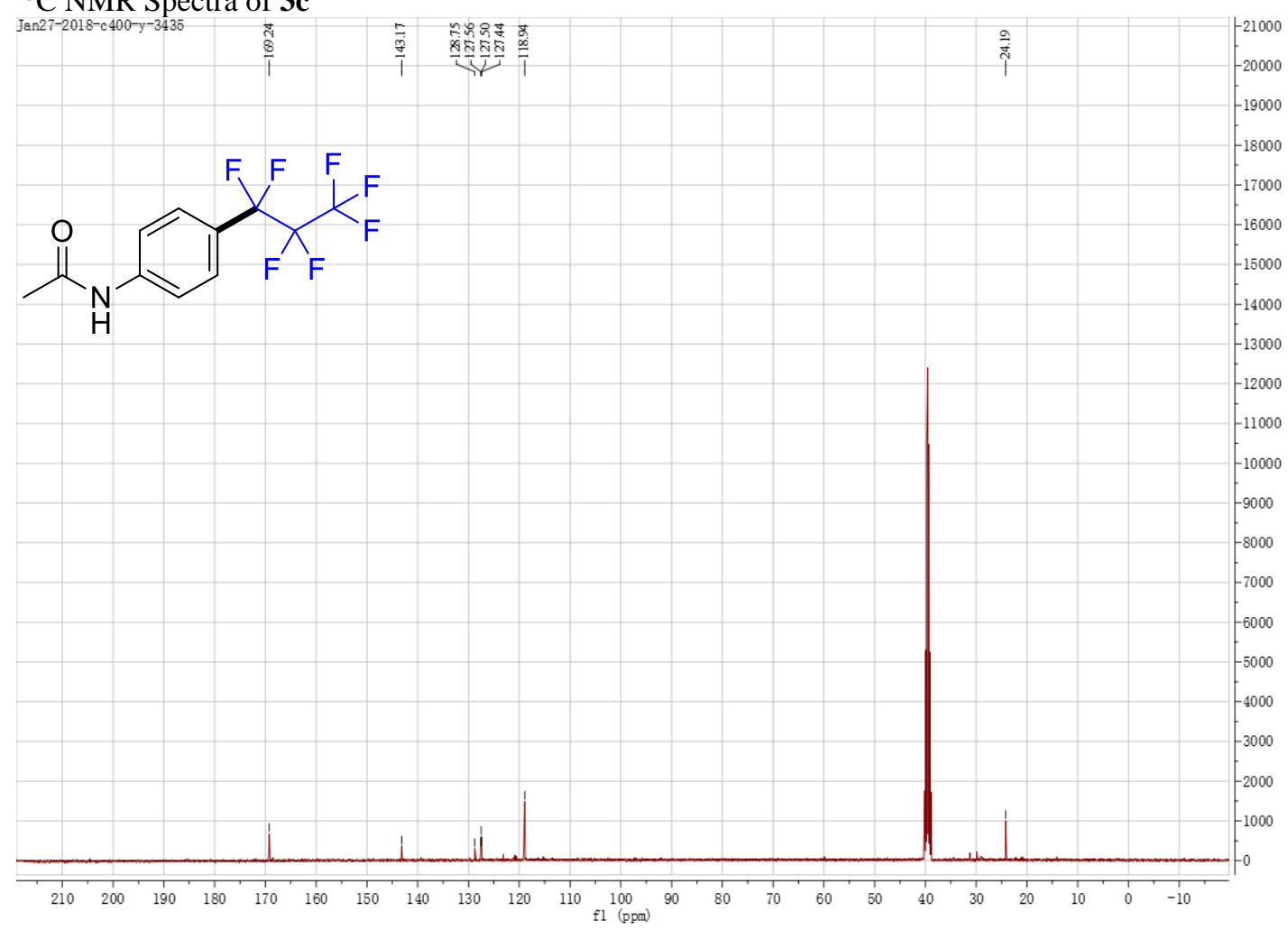

${ }^{1} \mathrm{H}$ NMR Spectra of $\mathbf{3 d}$

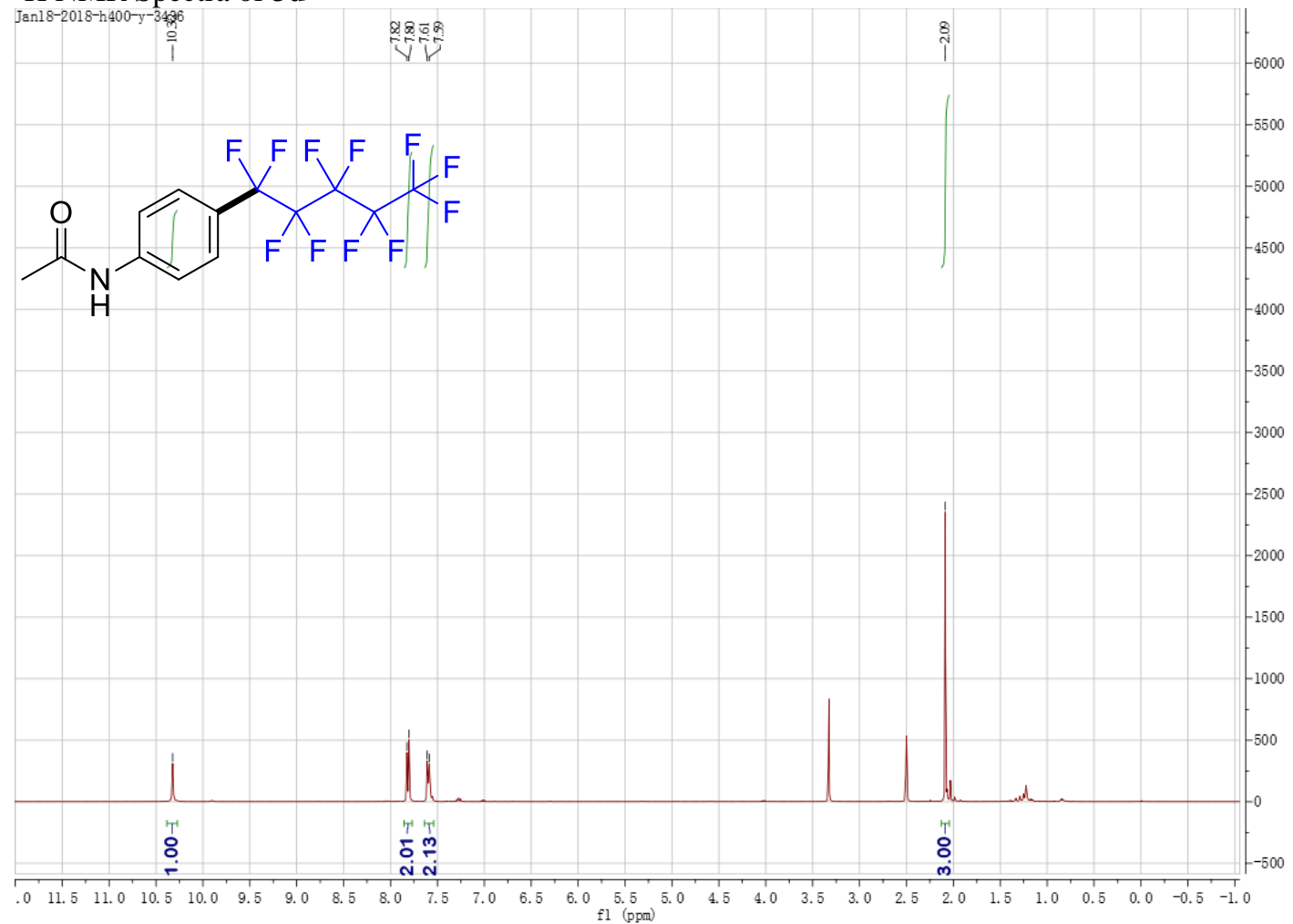


${ }_{\text {Jan27-2018-f } 400-y-3436}^{19}$ F NMR Spectra of 3d

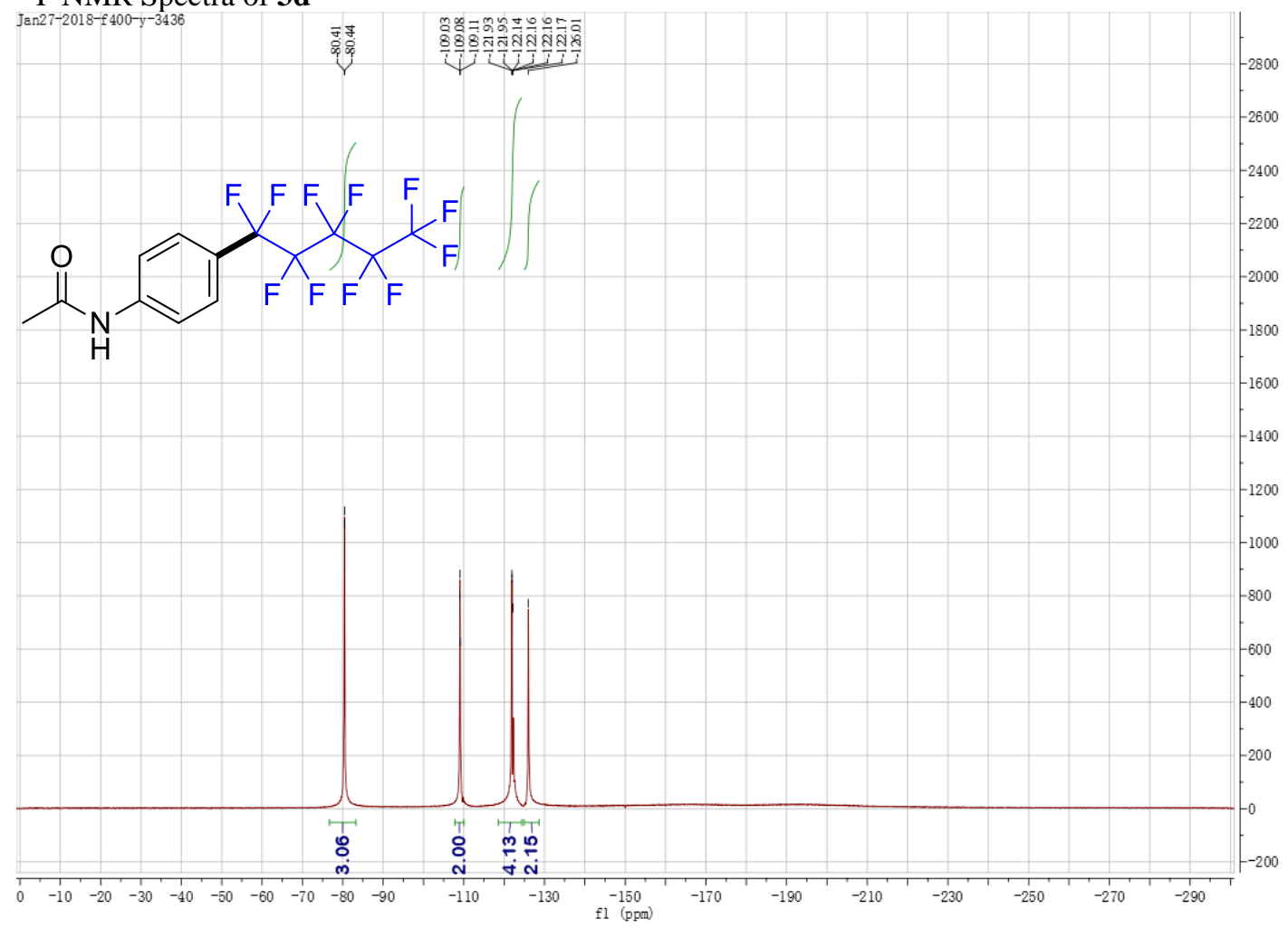

${ }^{13}$ C NMR Spectra of 3d

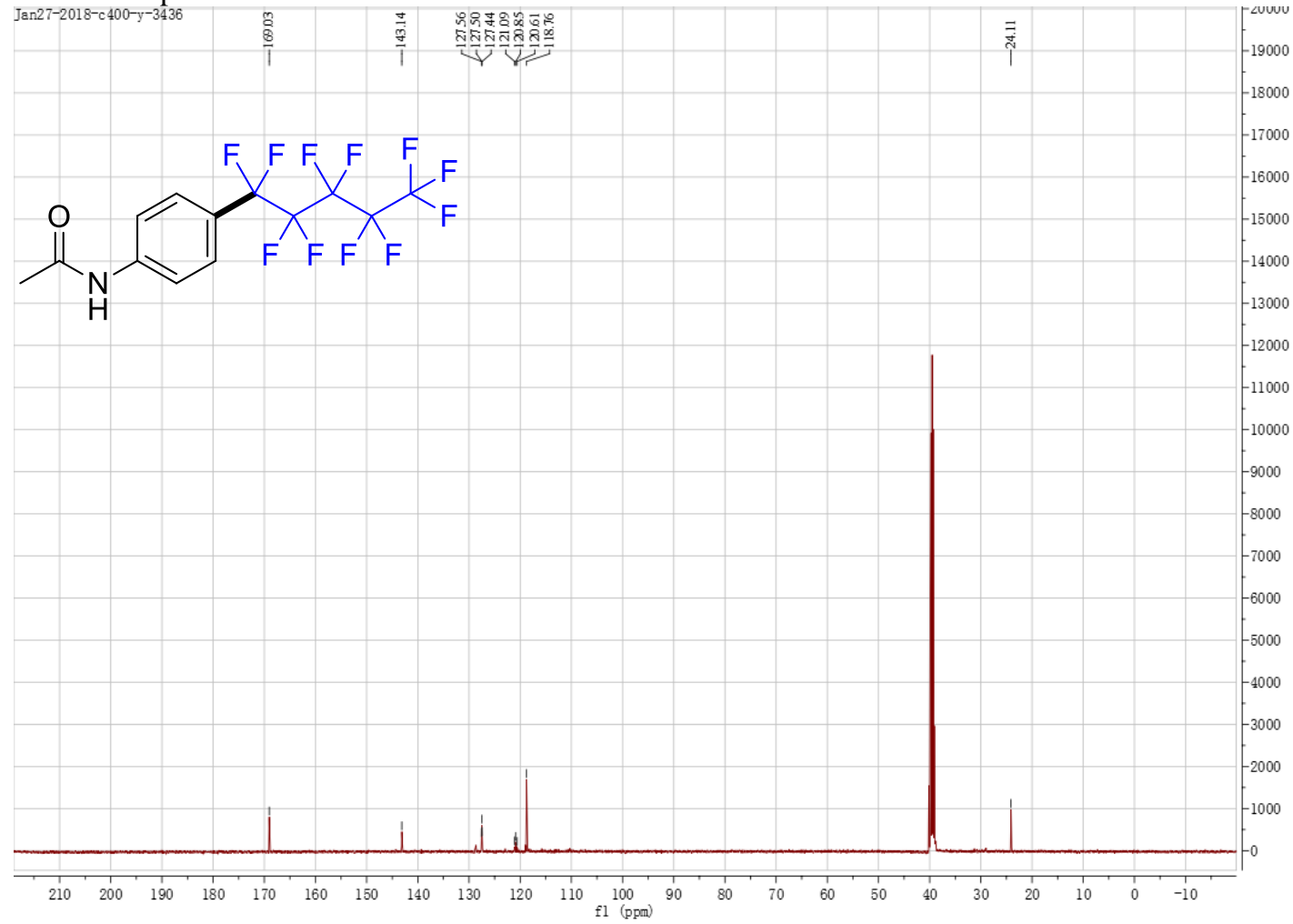


${ }^{1} \mathrm{H}$ NMR Spectra of $\mathbf{3 e}$

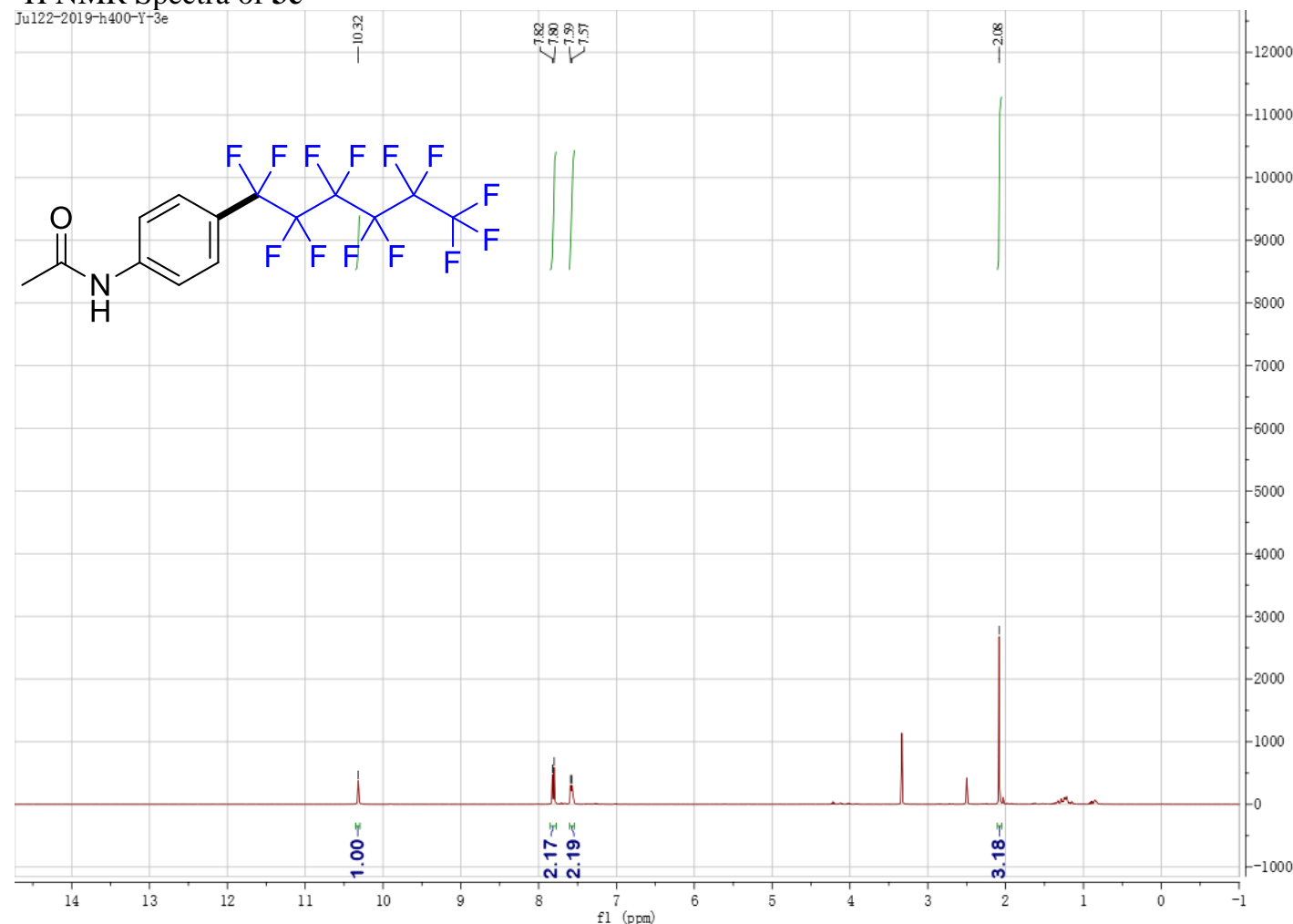

${ }^{19} \mathrm{~F}$ NMR Spectra of $\mathbf{3 e}$

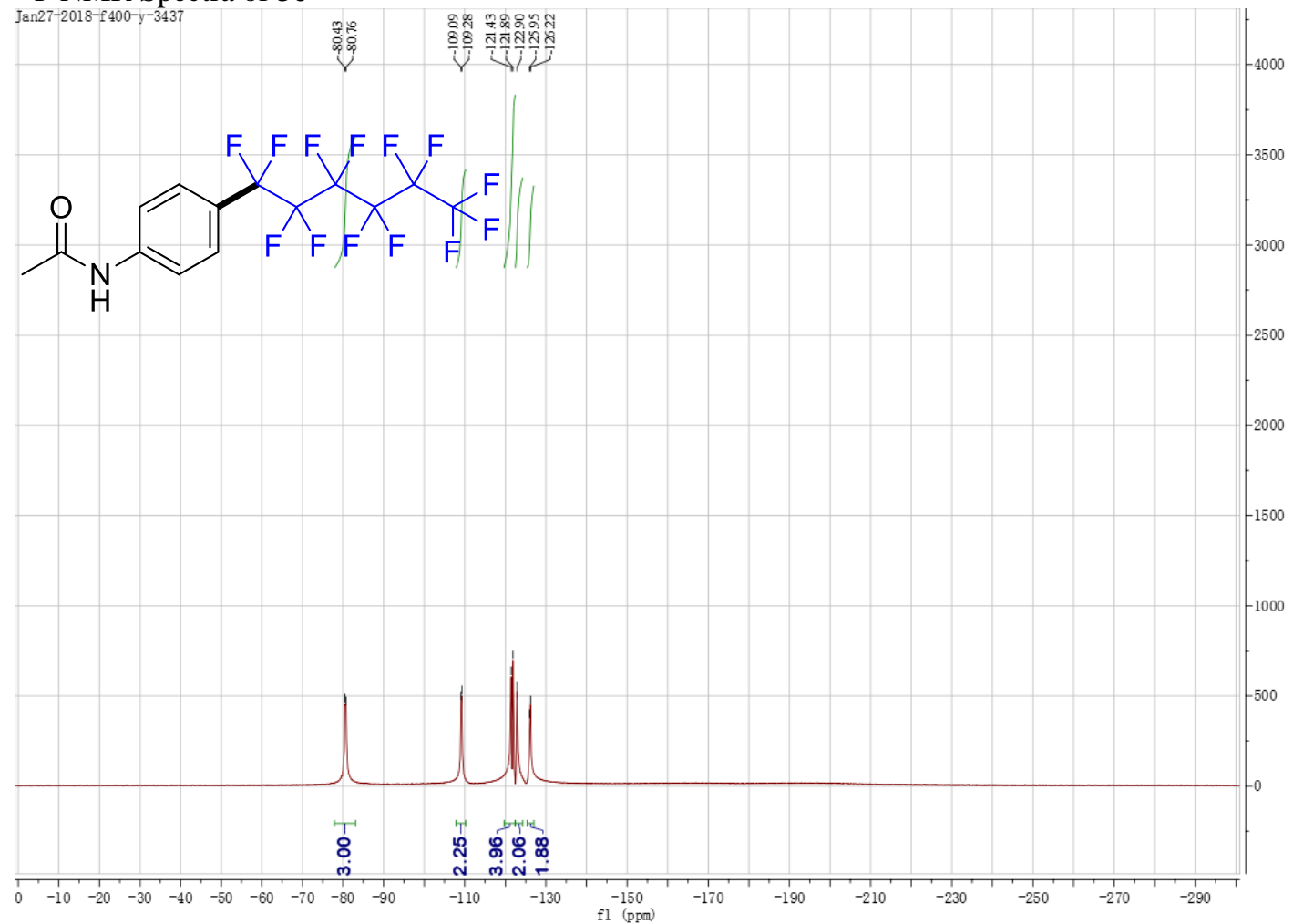


${ }^{13}$ C NMR Spectra of $\mathbf{3 e}$

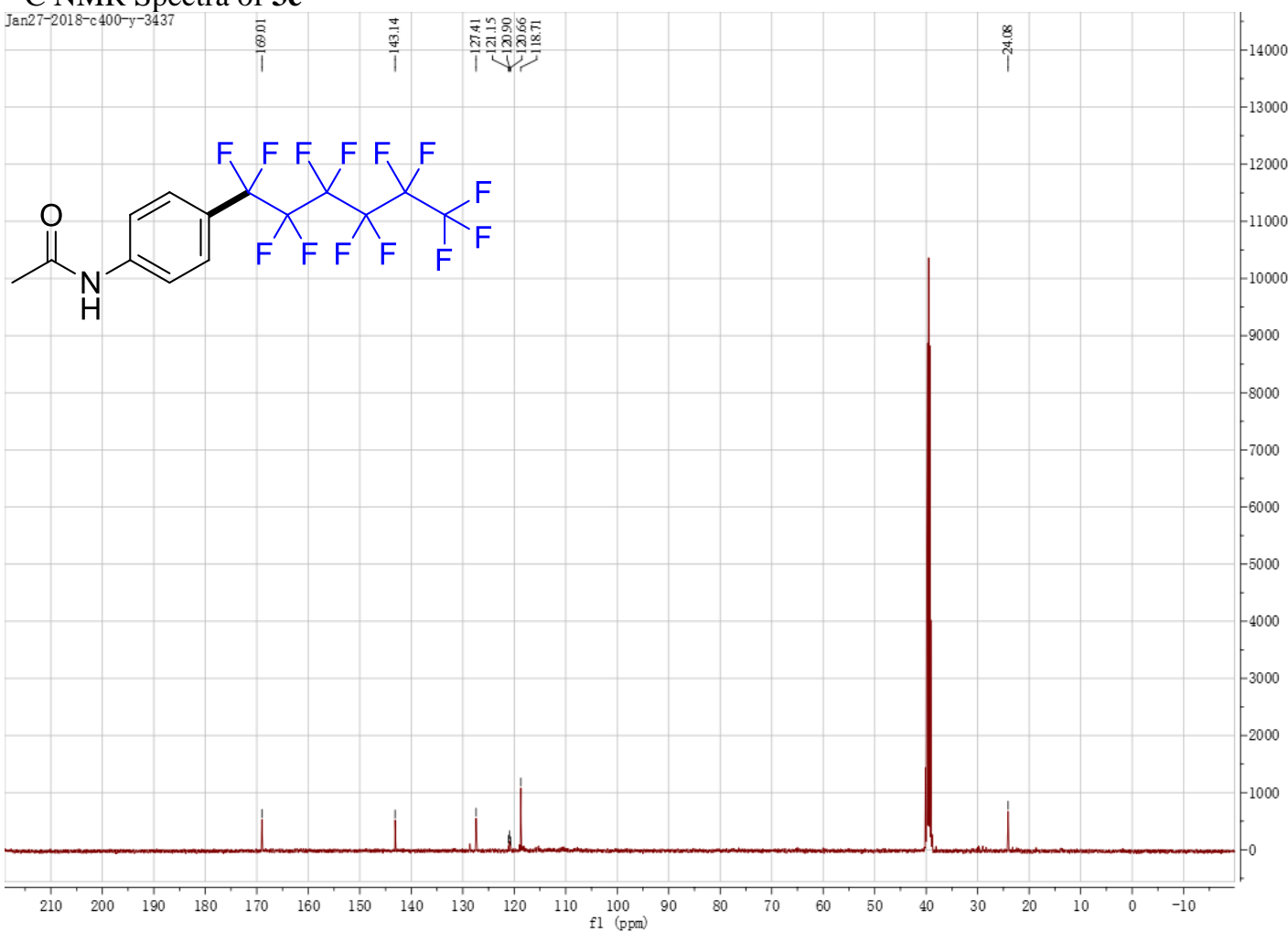

${ }^{1} \mathrm{H}$ NMR Spectra of $\mathbf{3 f}$

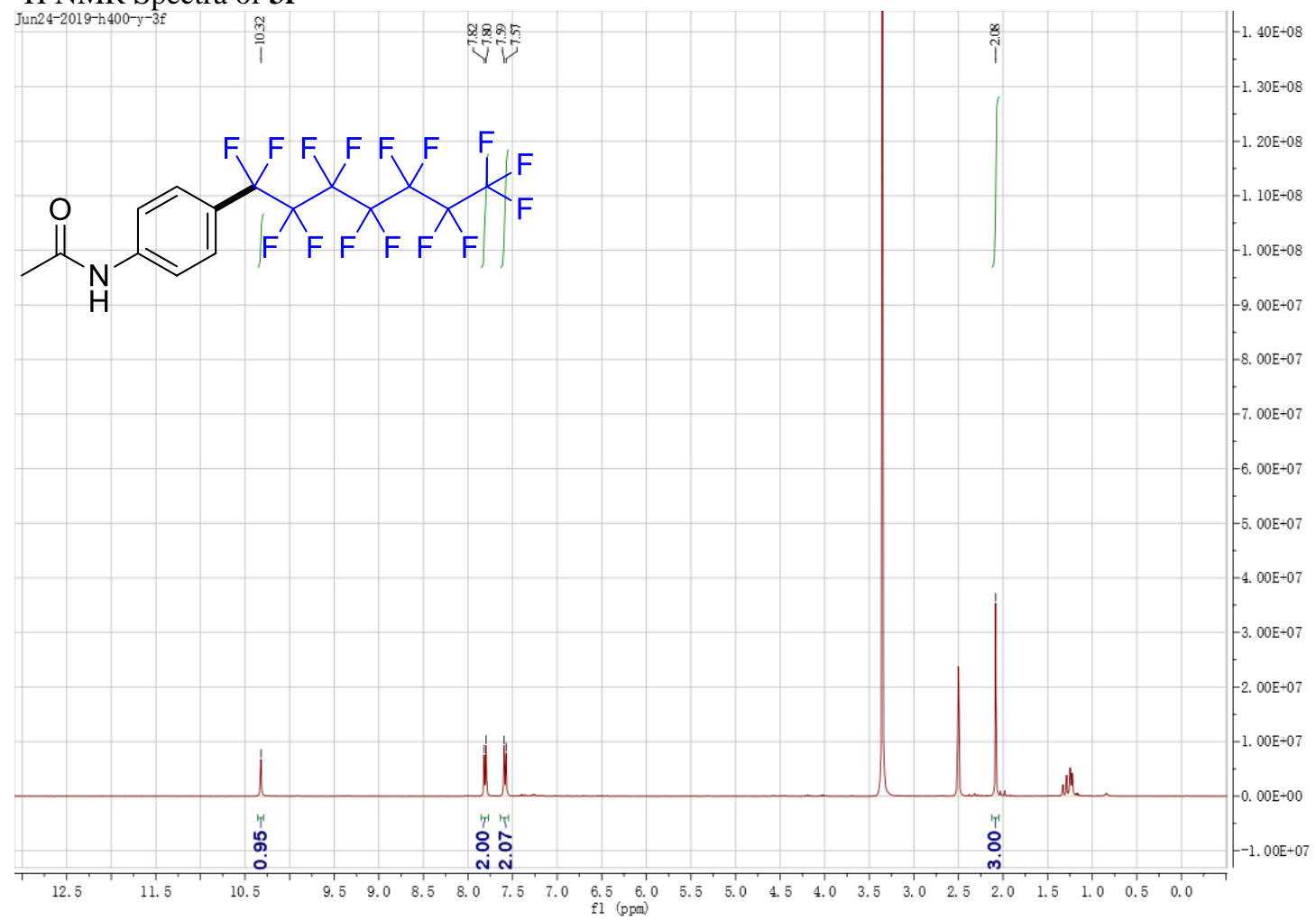


${ }_{\text {Jan27-2018-f400-y-3438 }}^{19}$ N NMR Spectra of $\mathbf{3 f}$

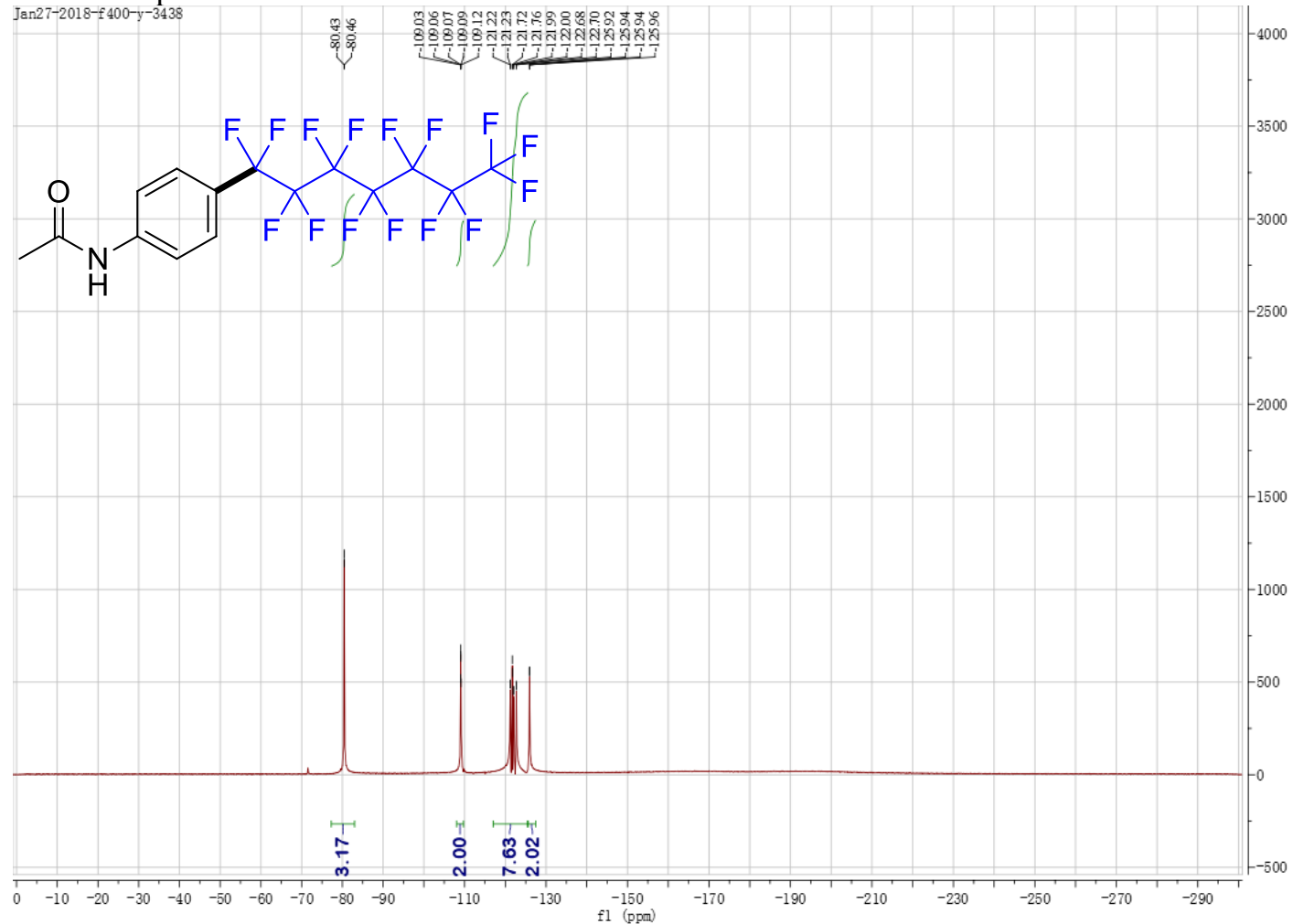

${ }^{13} \mathrm{C}$ NMR Spectra of $\mathbf{3 f}$

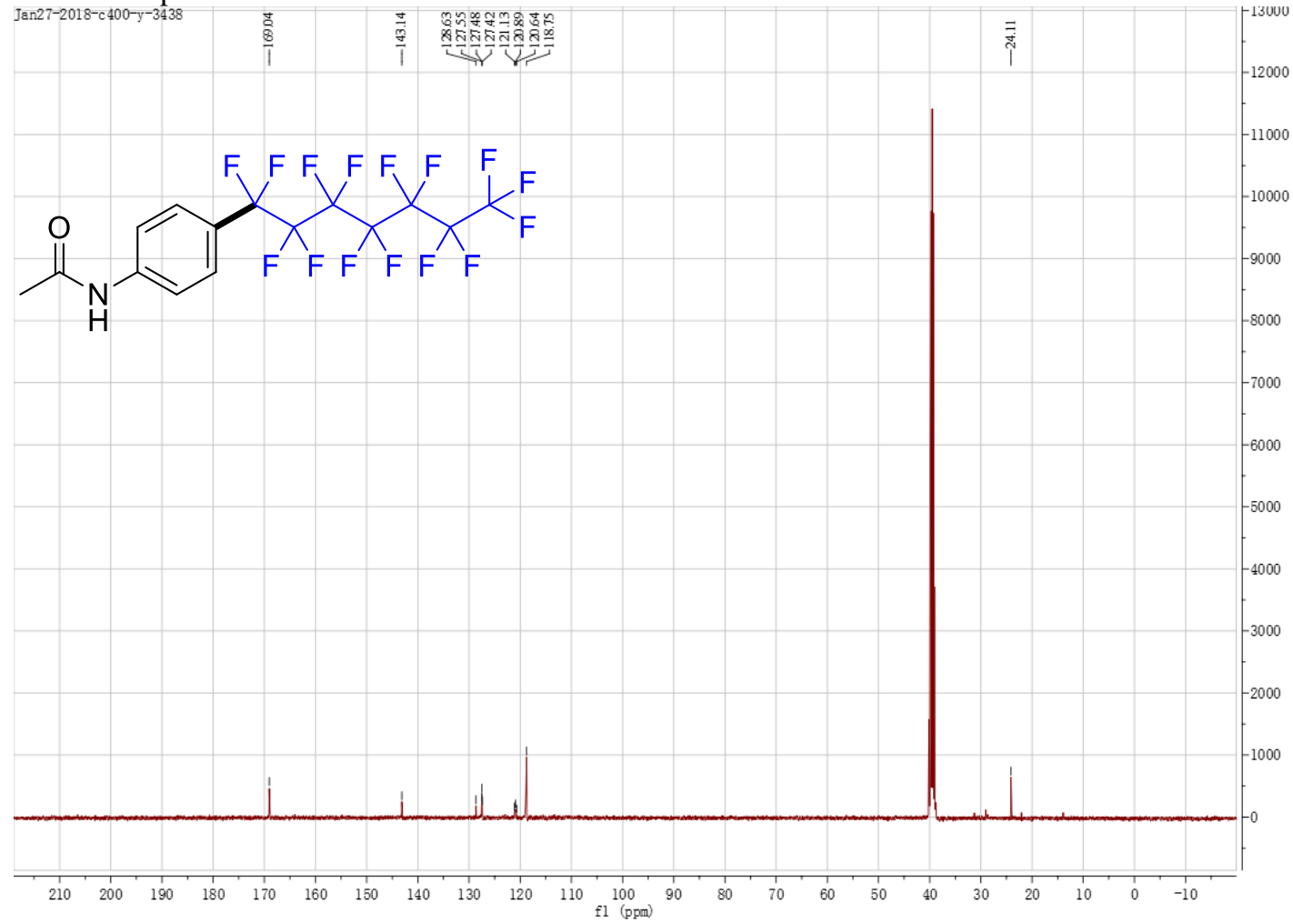


${ }^{1} \mathrm{H}$ NMR Spectra of $\mathbf{3 g}$

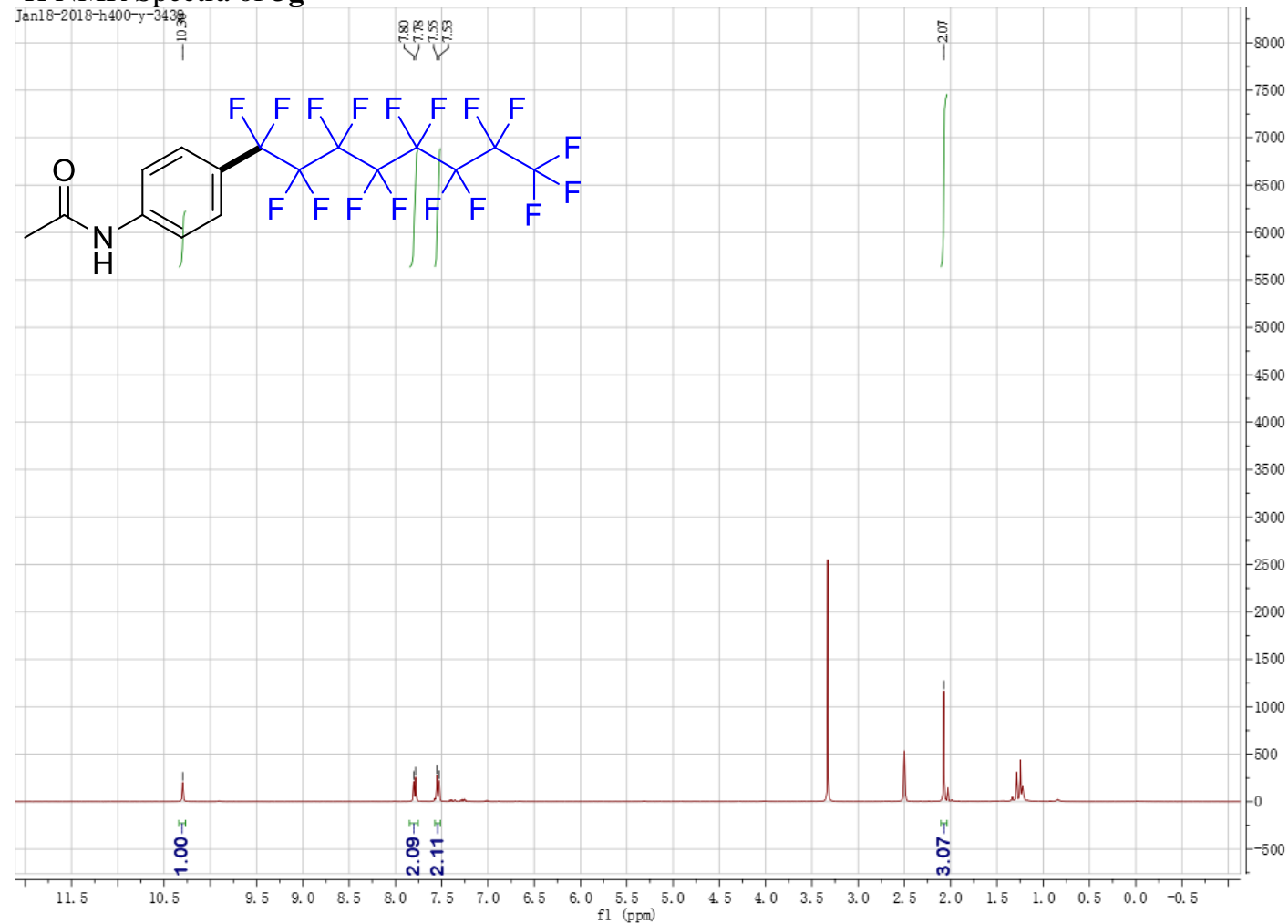

${ }^{19} \mathrm{~F}$ NMR Spectra of $\mathbf{3 g}$

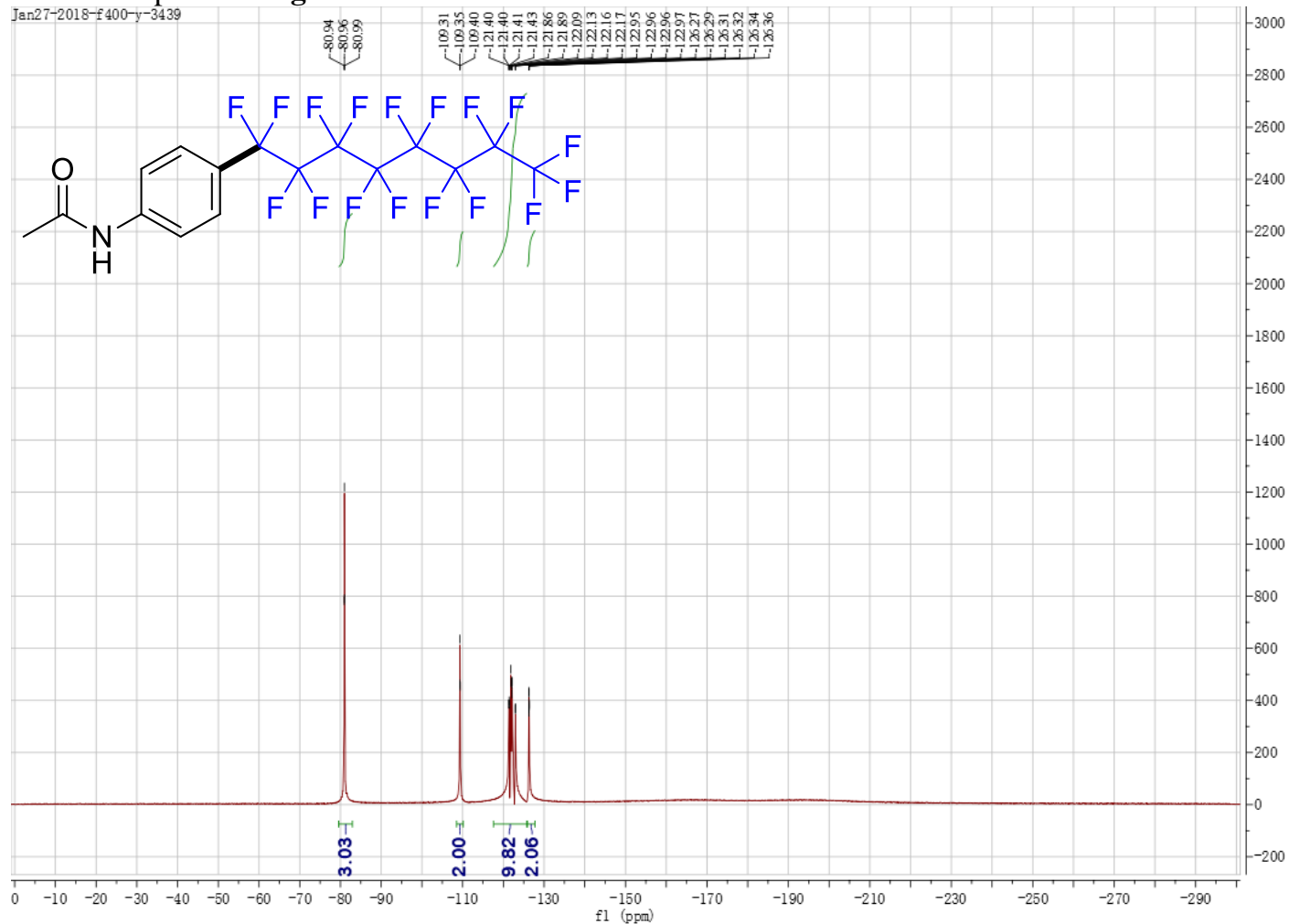


${ }^{13} \mathrm{C}$ NMR Spectra of $\mathbf{3 g}$

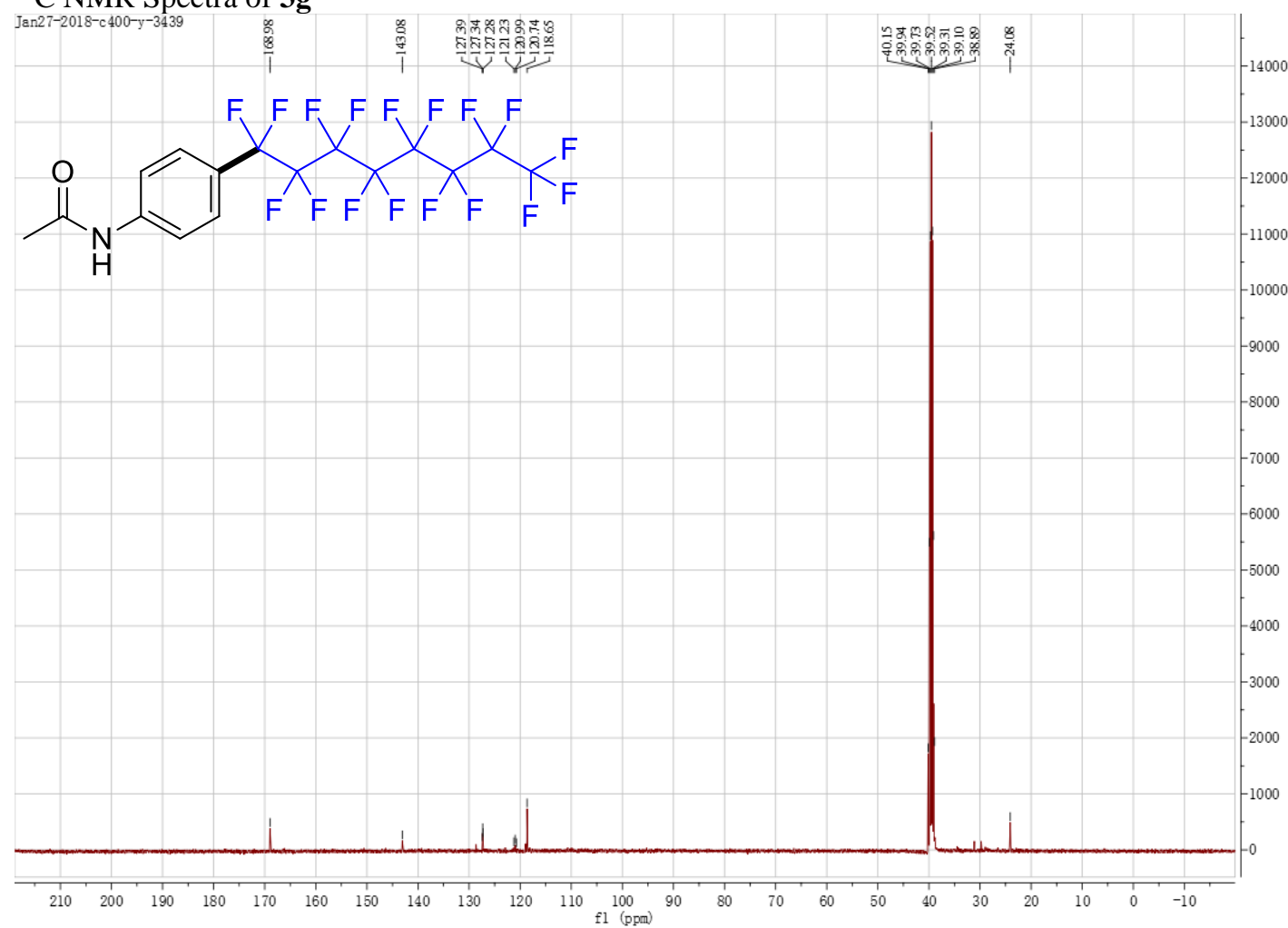

${ }^{1} \mathrm{H}$ NMR Spectra of $\mathbf{3 h}$ (Perfluorobenzene used as a co-solvent helps $\mathbf{3 h}$ dissolve in $\mathbf{d}_{6}-\mathrm{DMSO}$ )

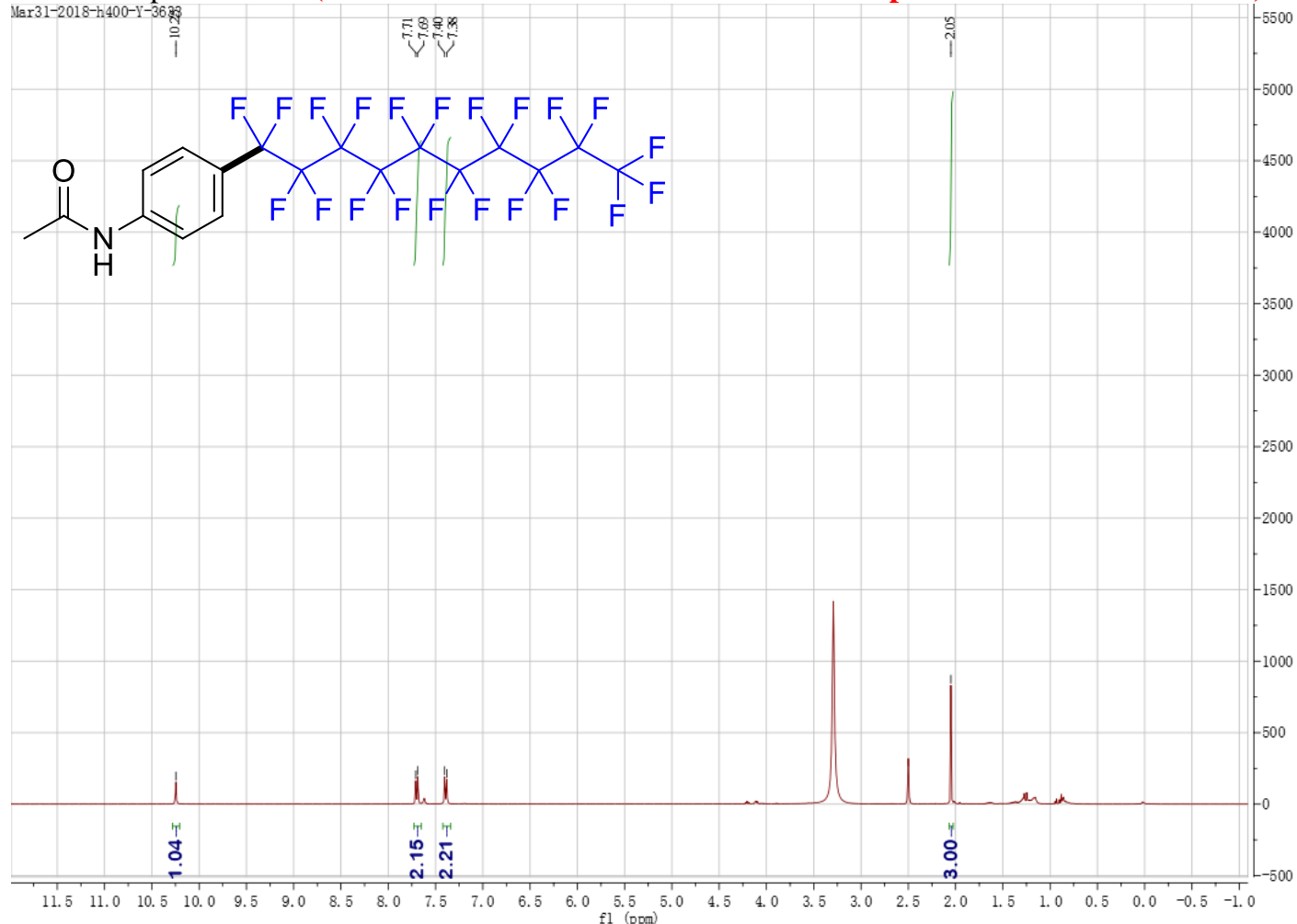


${ }^{19} \mathrm{~F}$ NMR Spectra of $\mathbf{3 h}$ (Perfluorobenzene used as a co-solvent helps $\mathbf{3 h}$ dissolve in $\mathrm{d}_{6}$-DMSO)

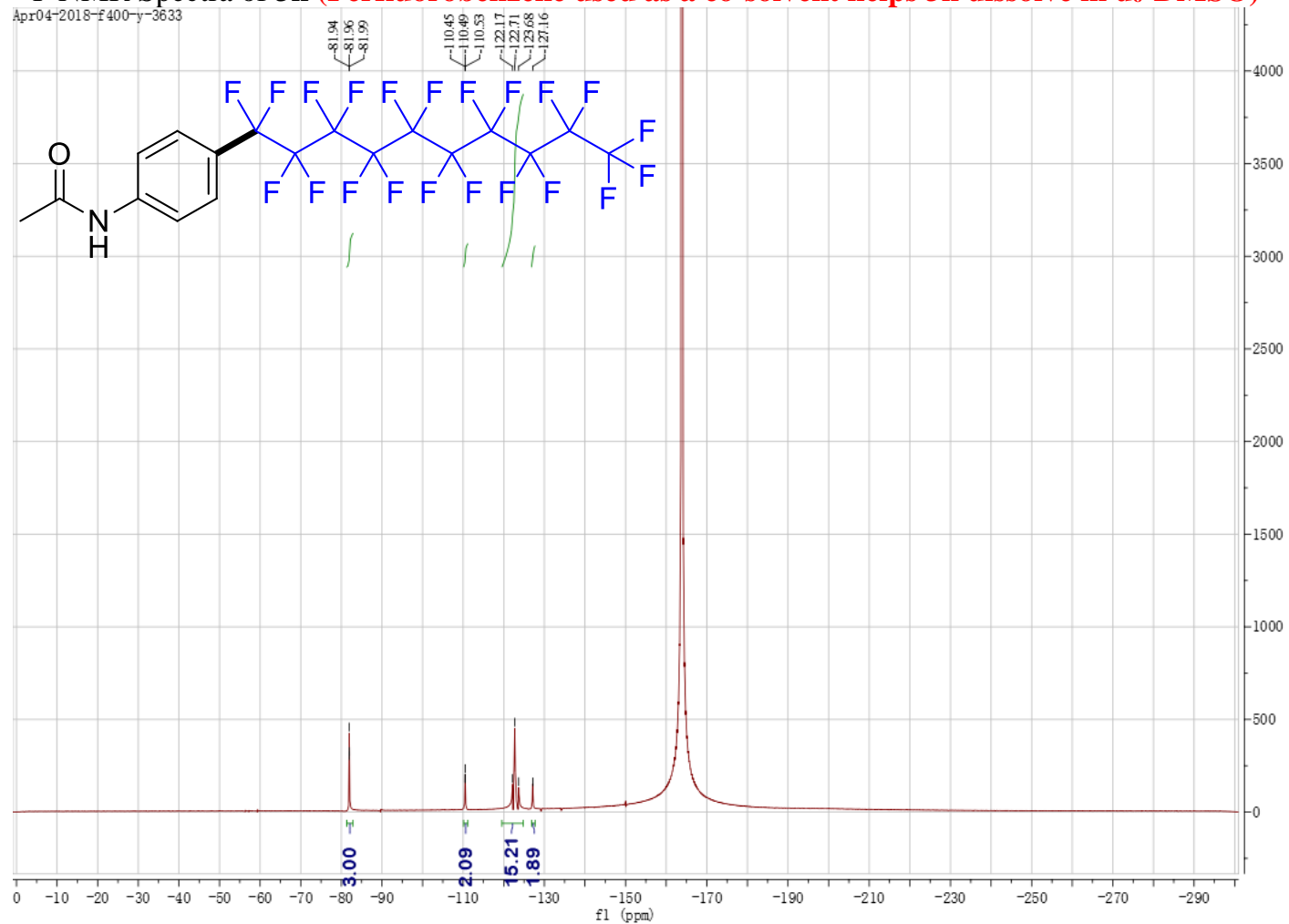

${ }^{13} \mathrm{C}$ NMR Spectra of $\mathbf{3 h}$ (Perfluorobenzene used as a co-solvent helps $\mathbf{3 h}$ dissolve in $\mathrm{d}_{6}$-DMSO)

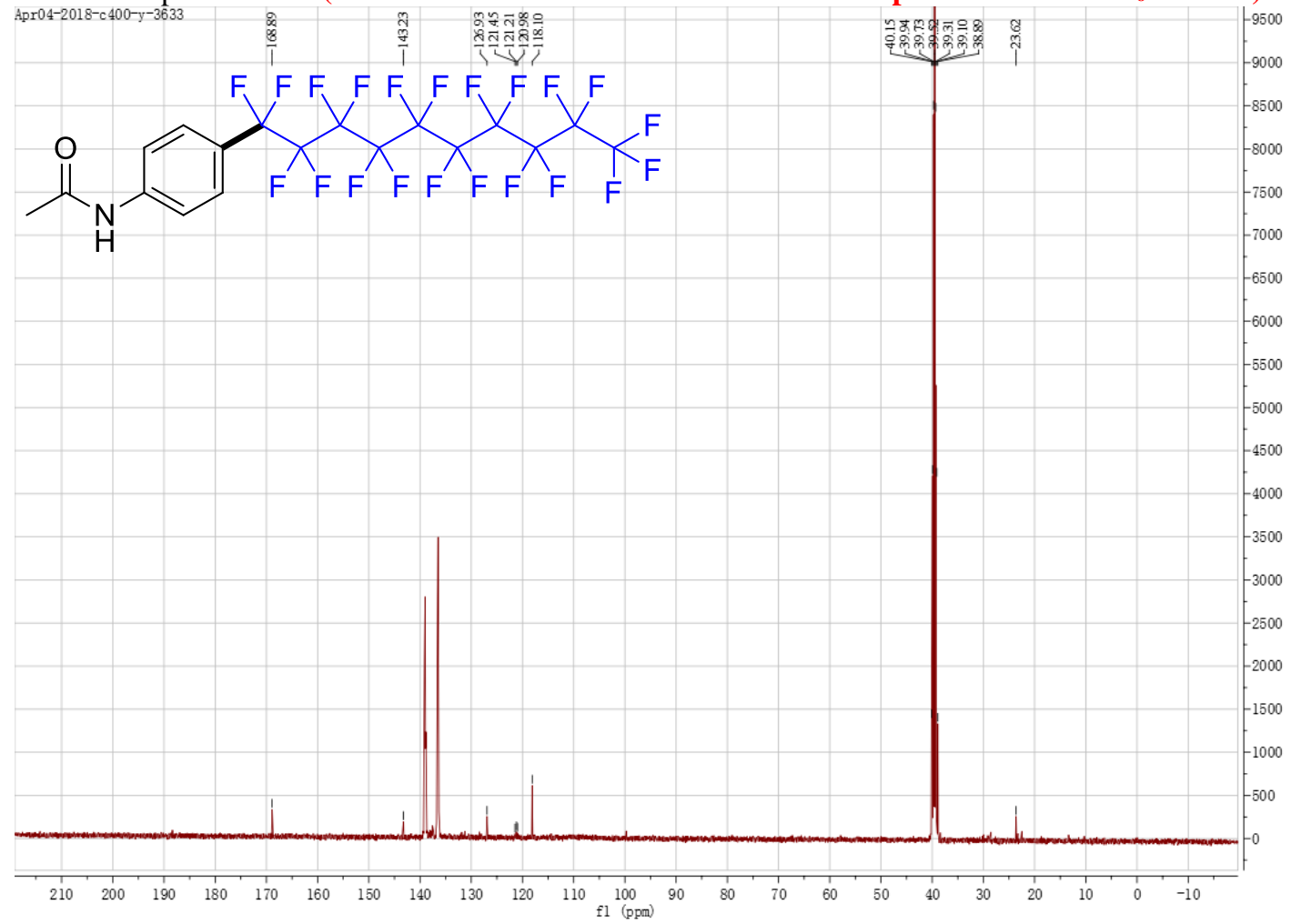


${ }^{1} \mathrm{H}$ NMR Spectra of $\mathbf{3 i}$

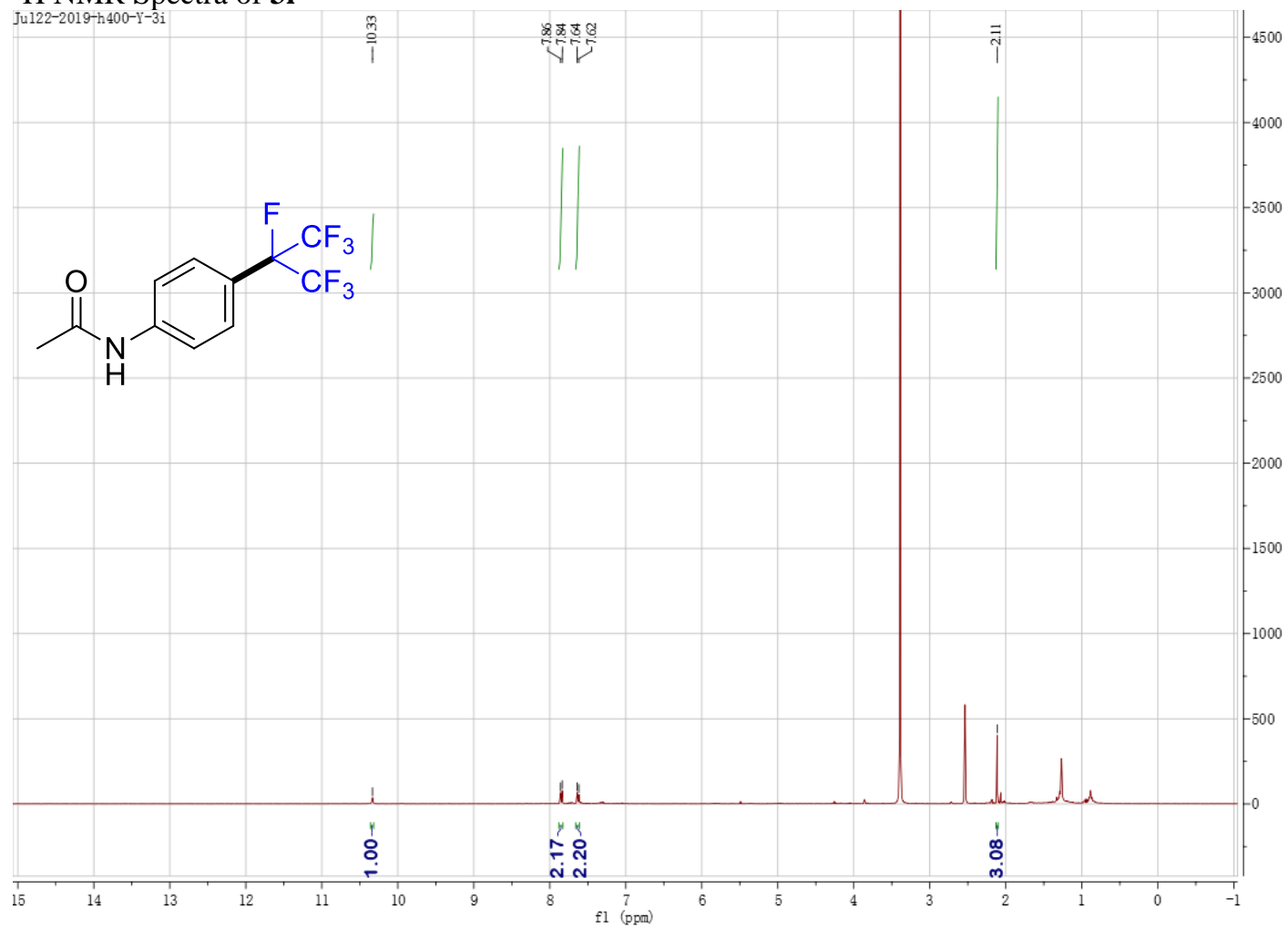

${ }^{19} \mathrm{~F}$ NMR Spectra of $\mathbf{3 i}$

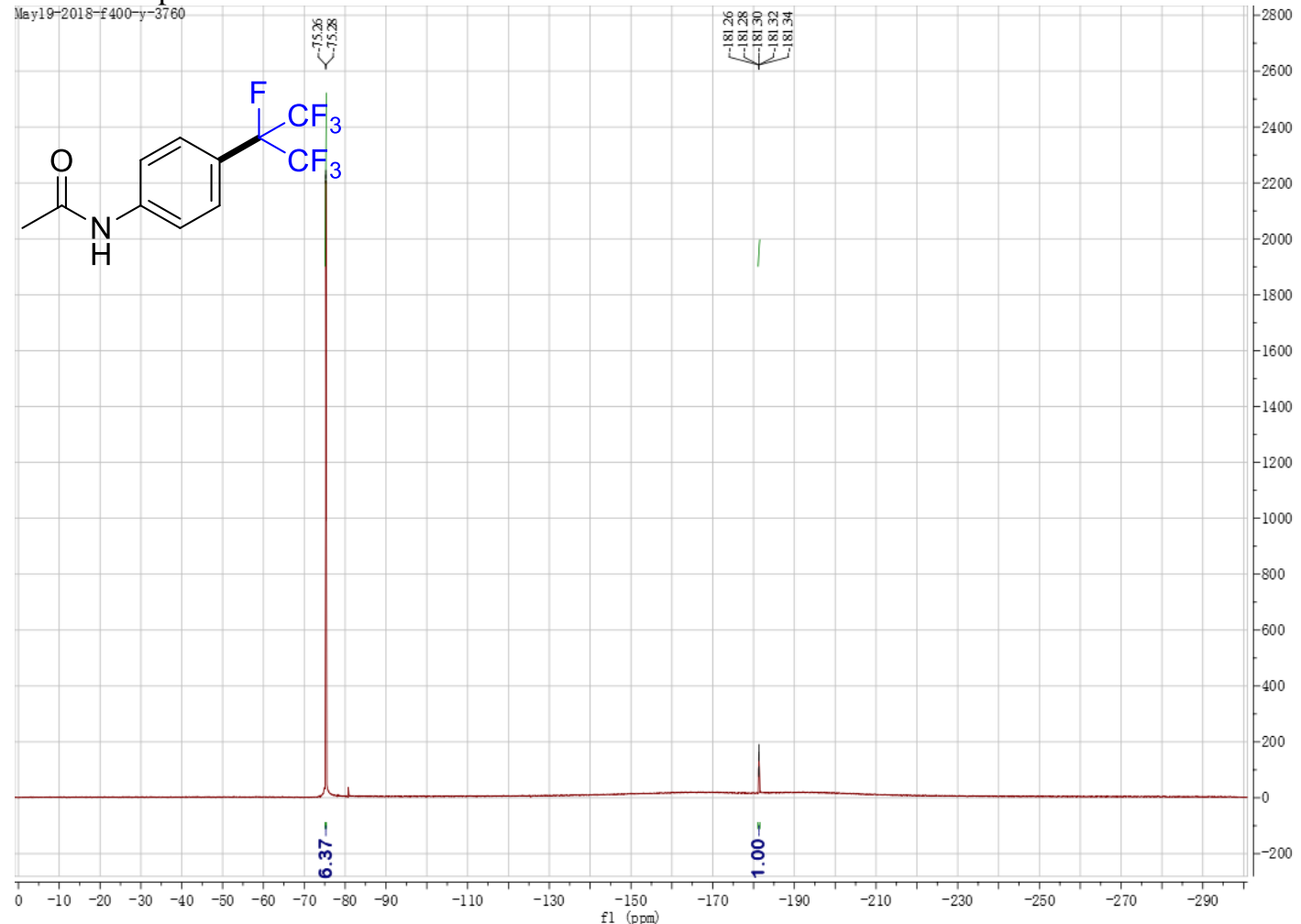


${ }^{13} \mathrm{C}$ NMR Spectra of $\mathbf{3 i}$

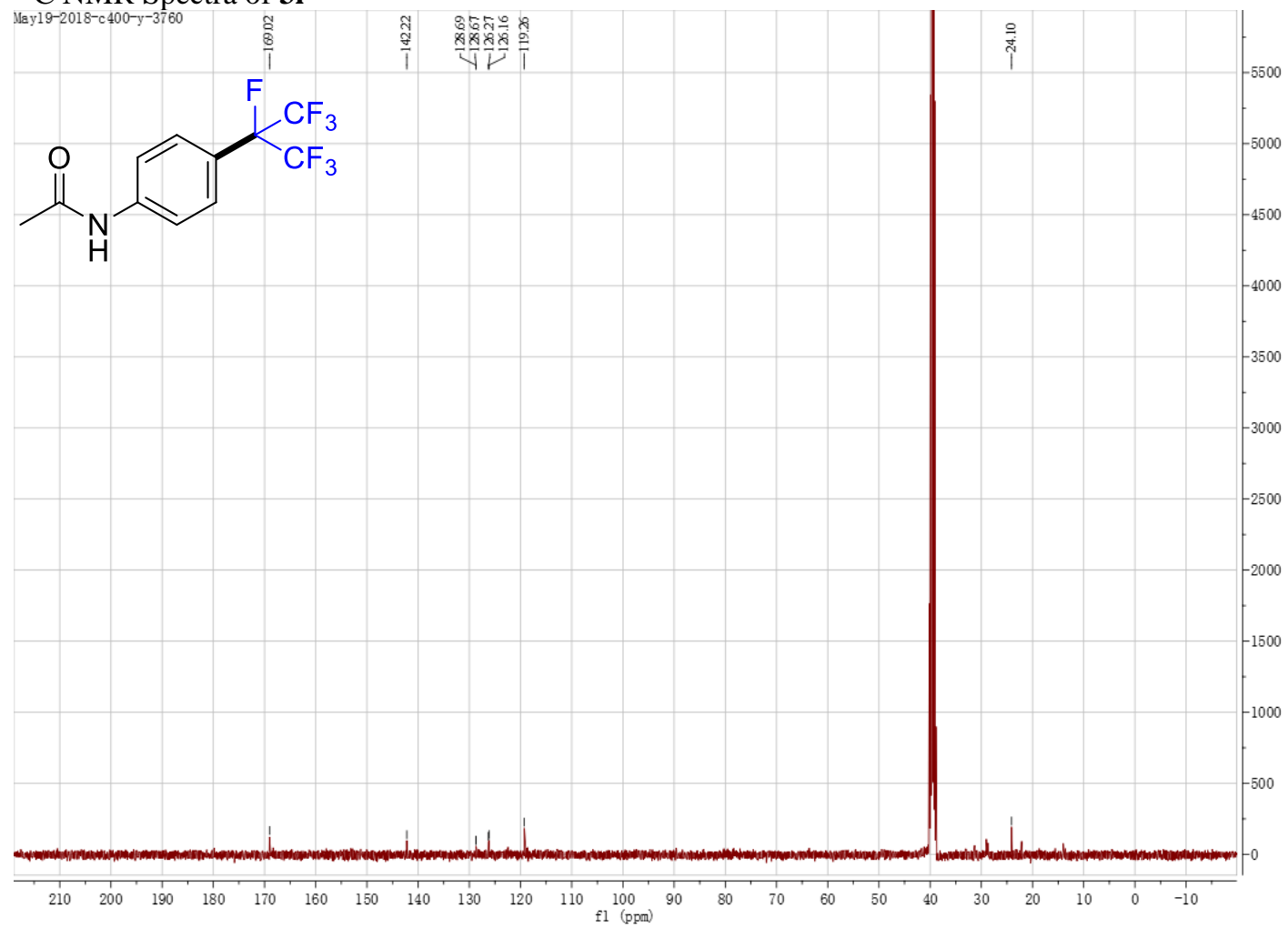

${ }^{1} \mathrm{H}$ NMR Spectra of $\mathbf{3 j}$ Ju122-2019-h400-Y-3.

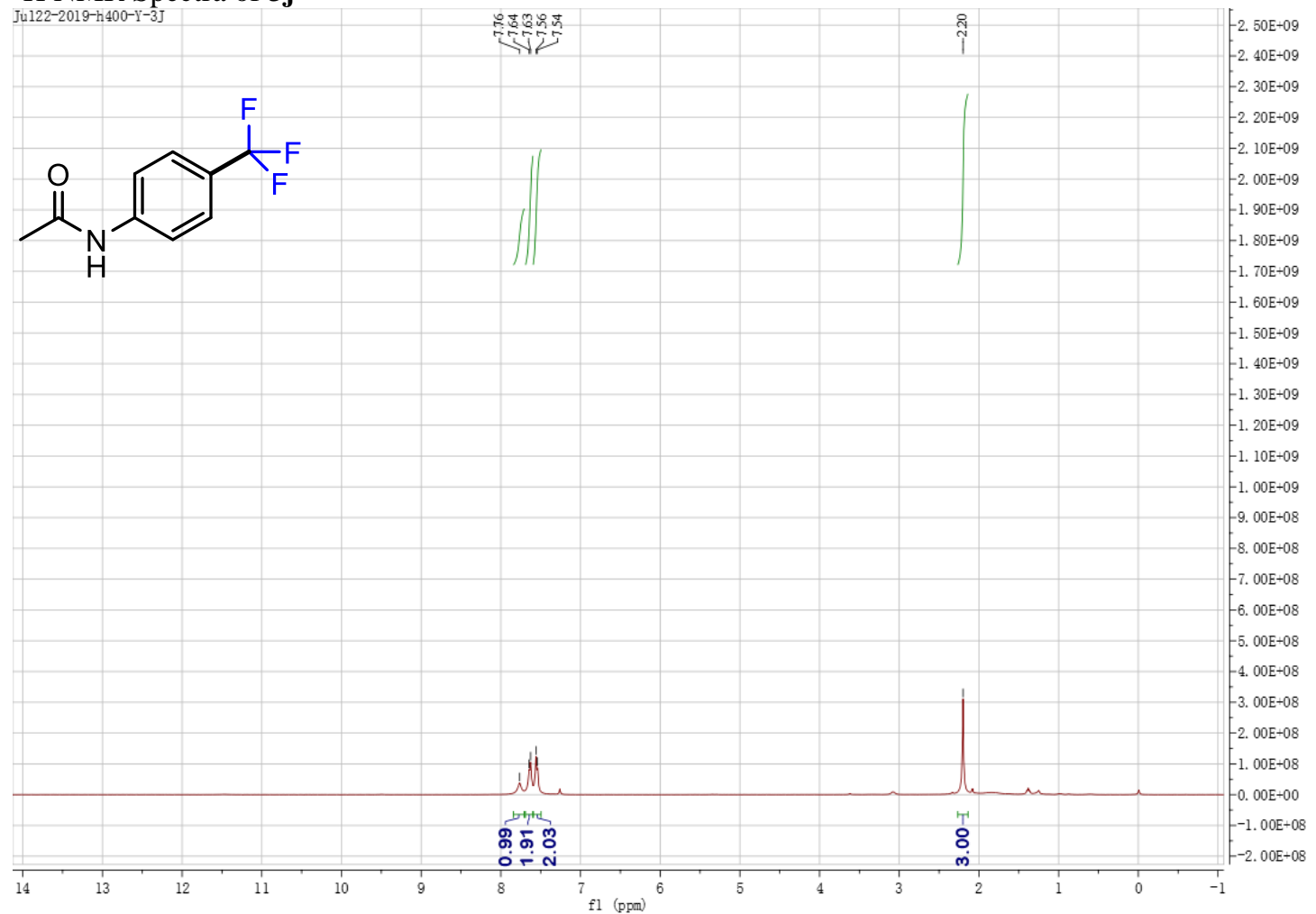


${ }^{19} \mathrm{~F}$ NMR Spectra of $\mathbf{3 j}$

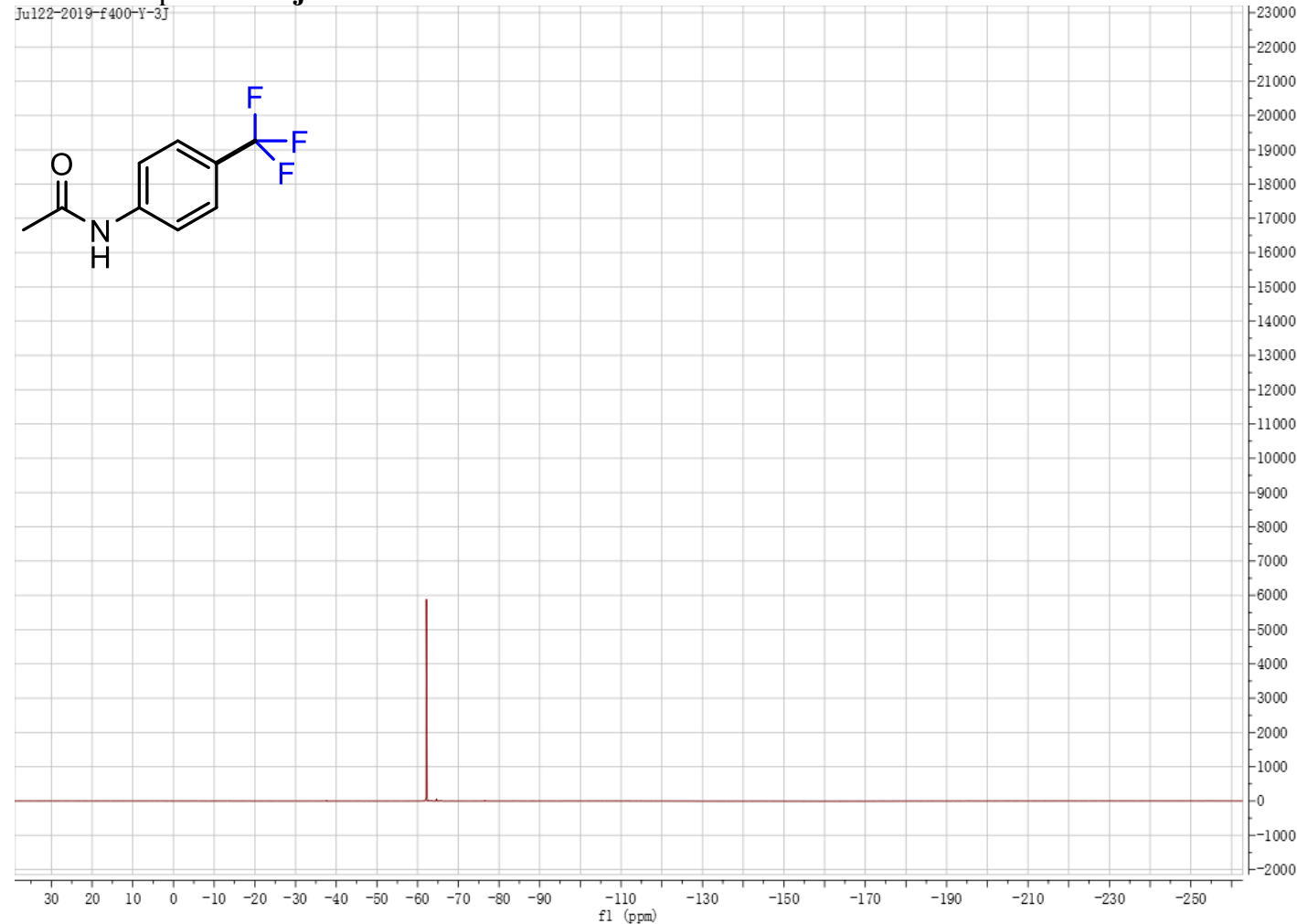

${ }^{13}$ C NMR Spectra of $\mathbf{3 j}$

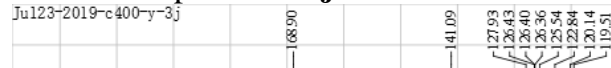<smiles>CC(=O)Nc1ccc(C(F)(F)F)cc1</smiles>
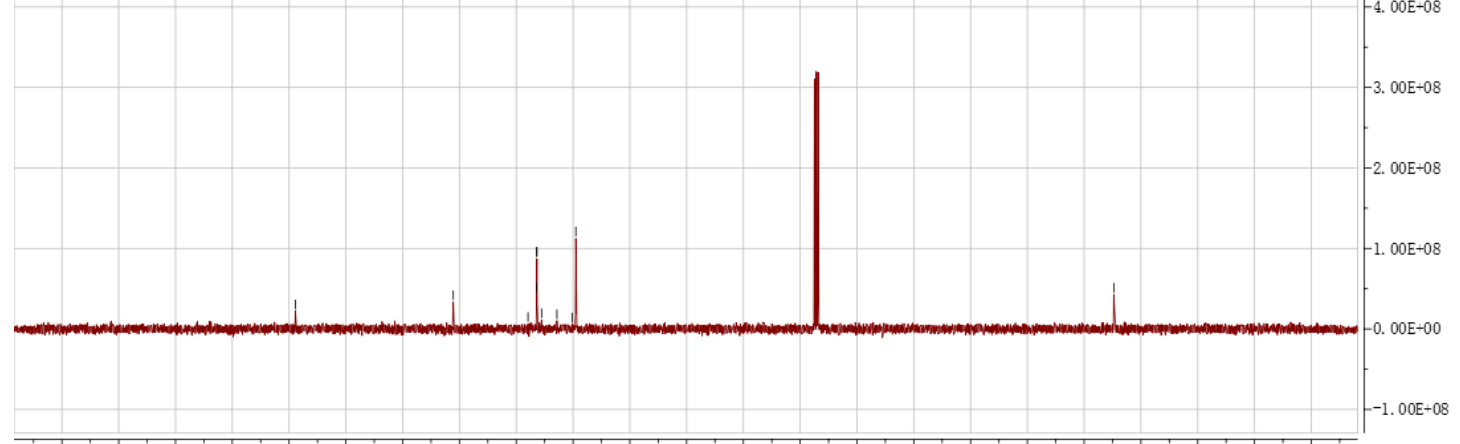

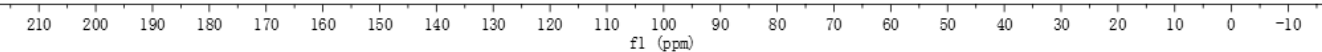


${ }^{1} \mathrm{H}$ NMR, ${ }^{19} \mathrm{~F}$ NMR \& ${ }^{13} \mathrm{C}$ NMR Spectra of $\mathbf{4 a}$

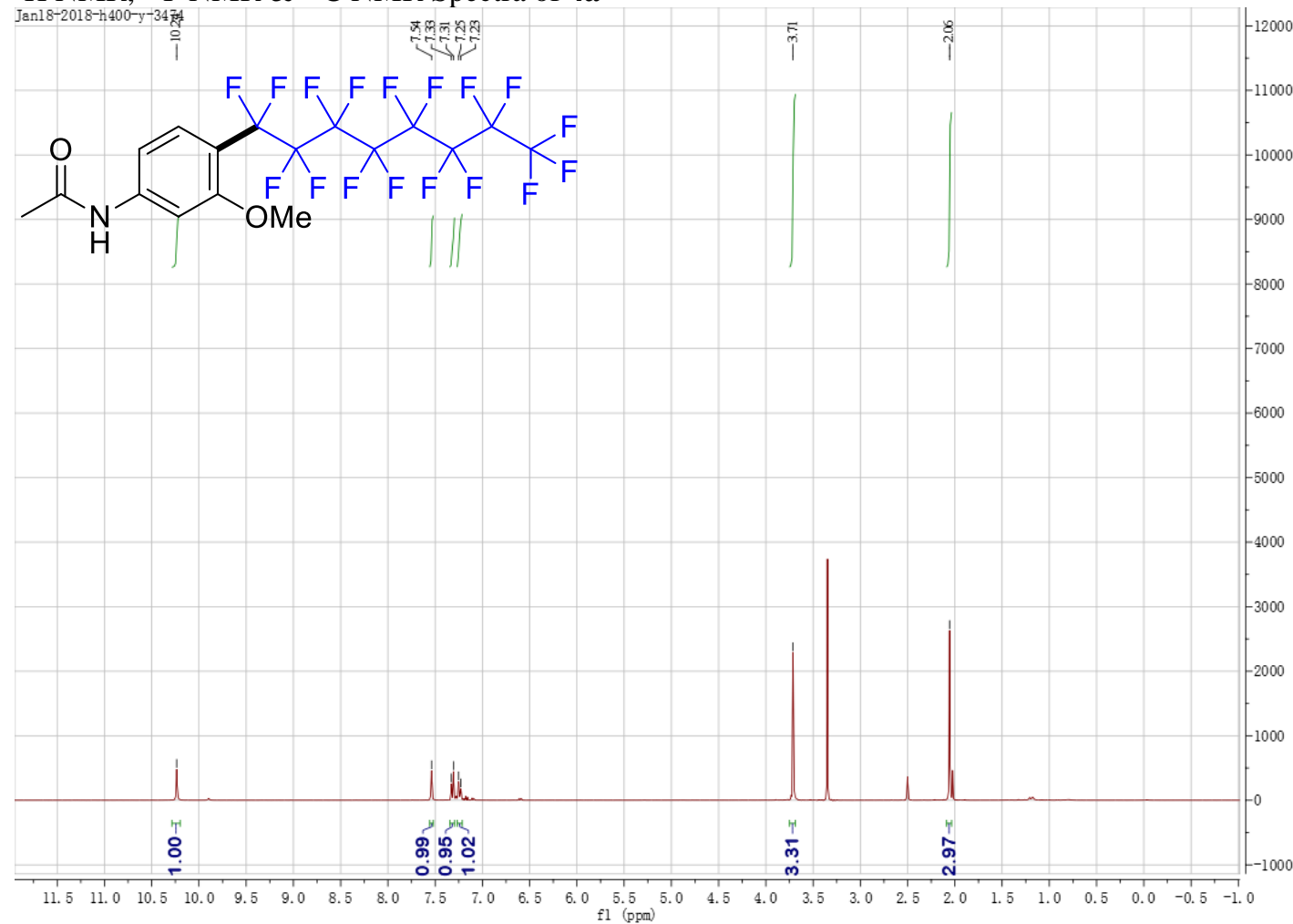

${ }^{19} \mathrm{~F}$ NMR Spectra of $\mathbf{4 a}$

Jan27-2018-f400-y-3474

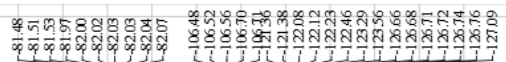
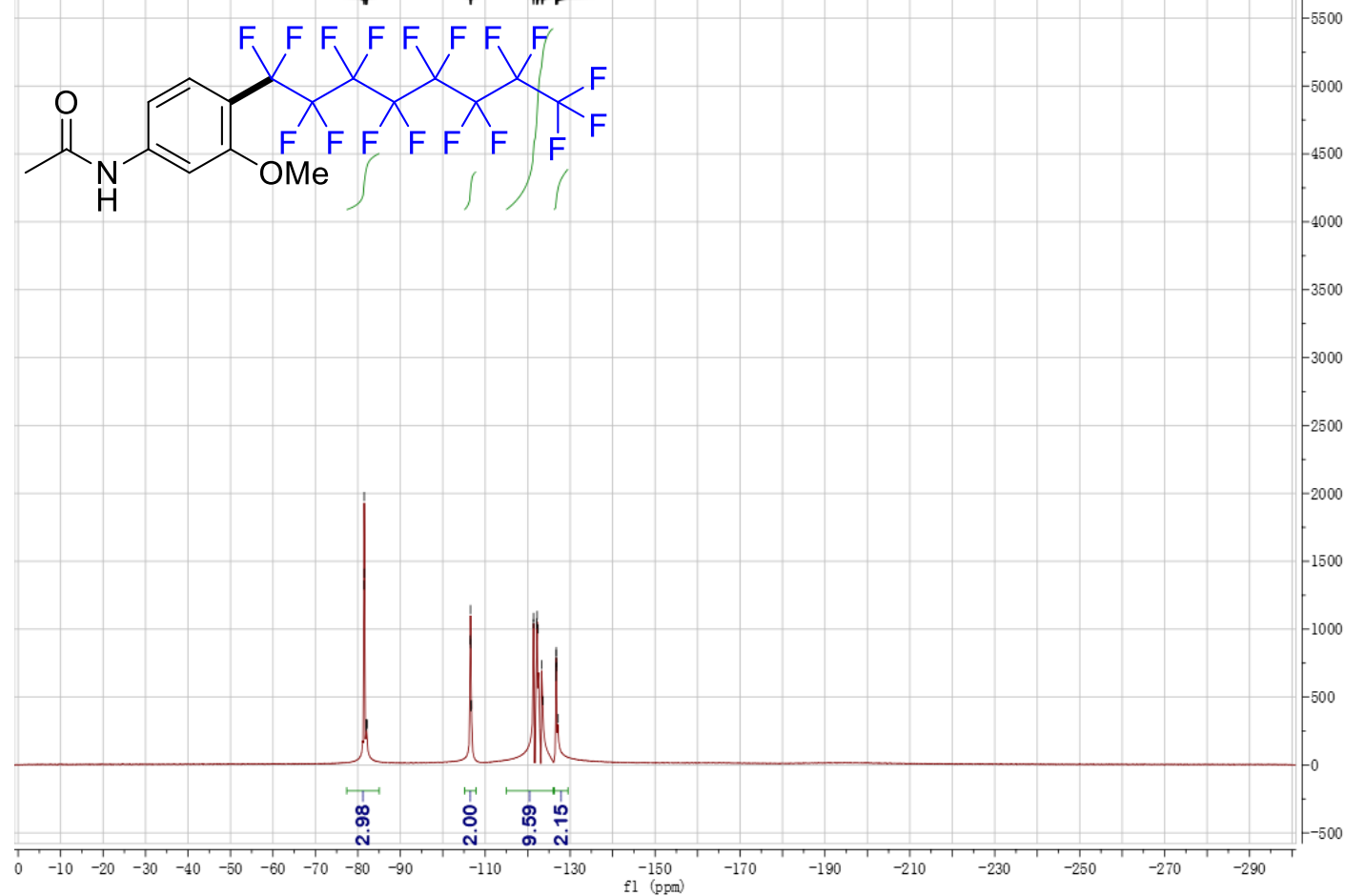
${ }^{13}$ C NMR Spectra of $\mathbf{4 a}$

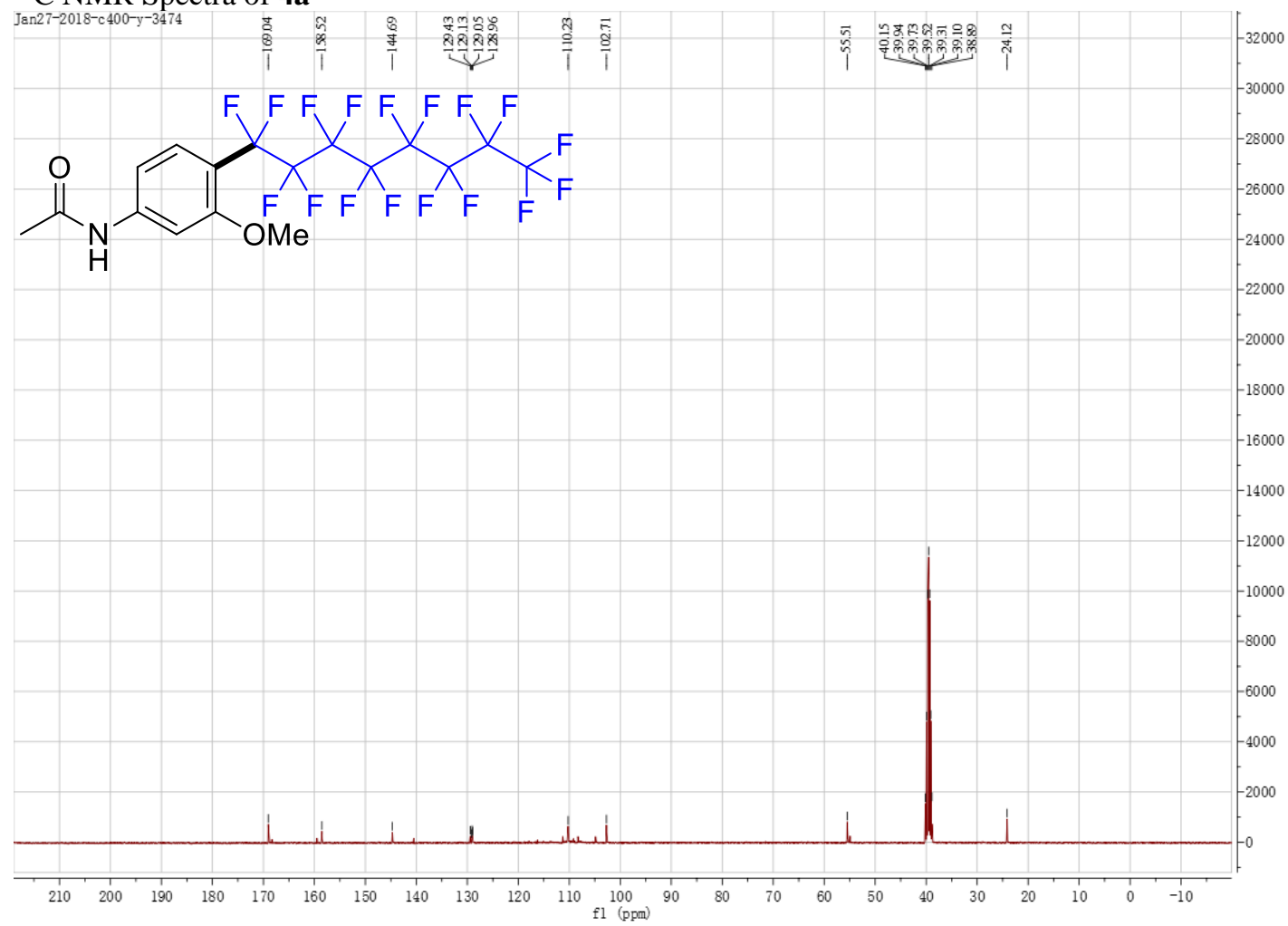

${ }^{1} \mathrm{H}$ NMR Spectra of $\mathbf{4 b}$ (Perfluorobenzene used as a co-solvent helps $4 \mathbf{b}$ dissolve in $\mathrm{d}_{6}$-DMSO)

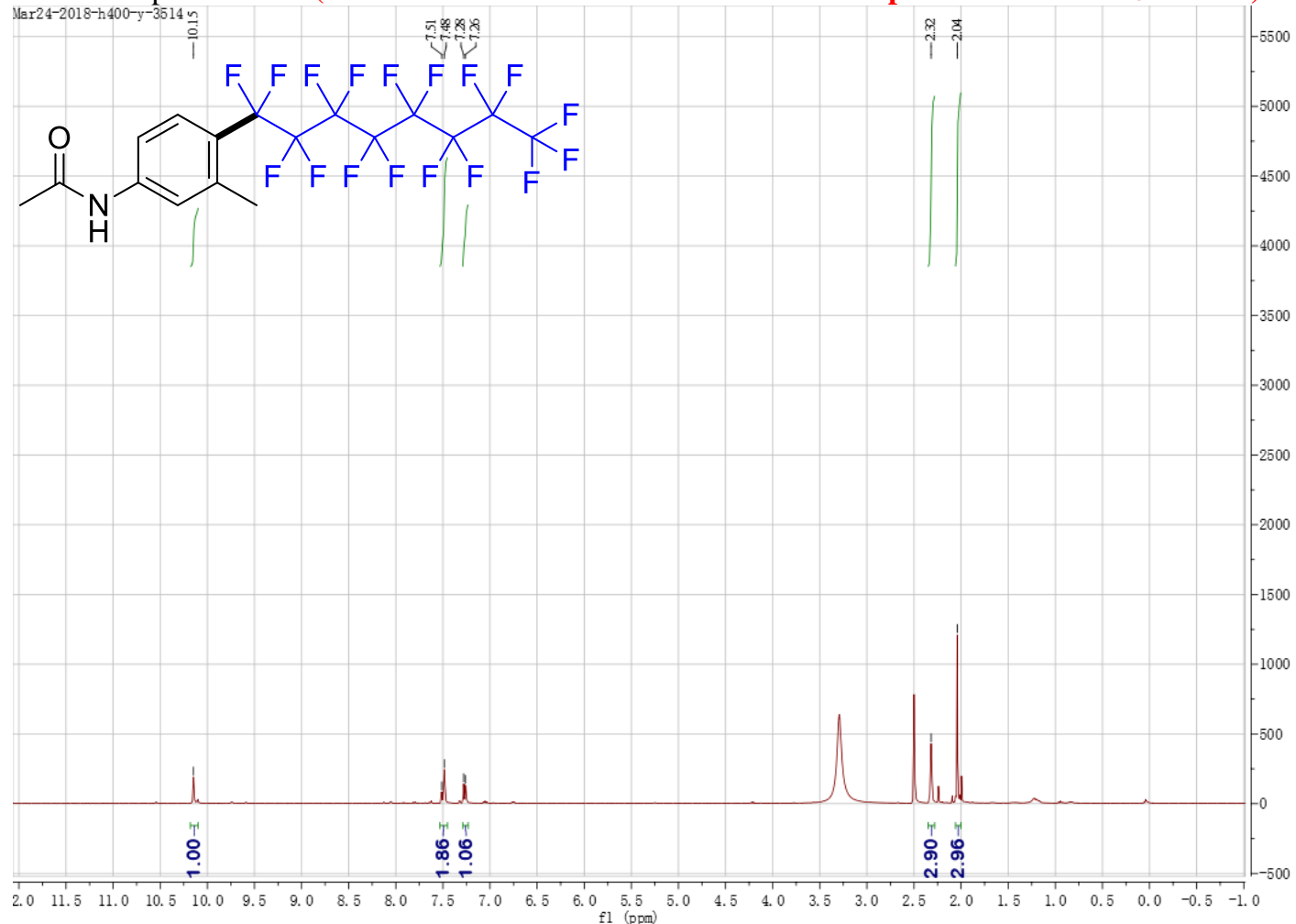


${ }^{19} \mathrm{~F}$ NMR Spectra of $\mathbf{4 b}$ (Perfluorobenzene used as a co-solvent helps $4 \mathrm{~b}$ dissolve in $\mathrm{d}_{6}$-DMSO)

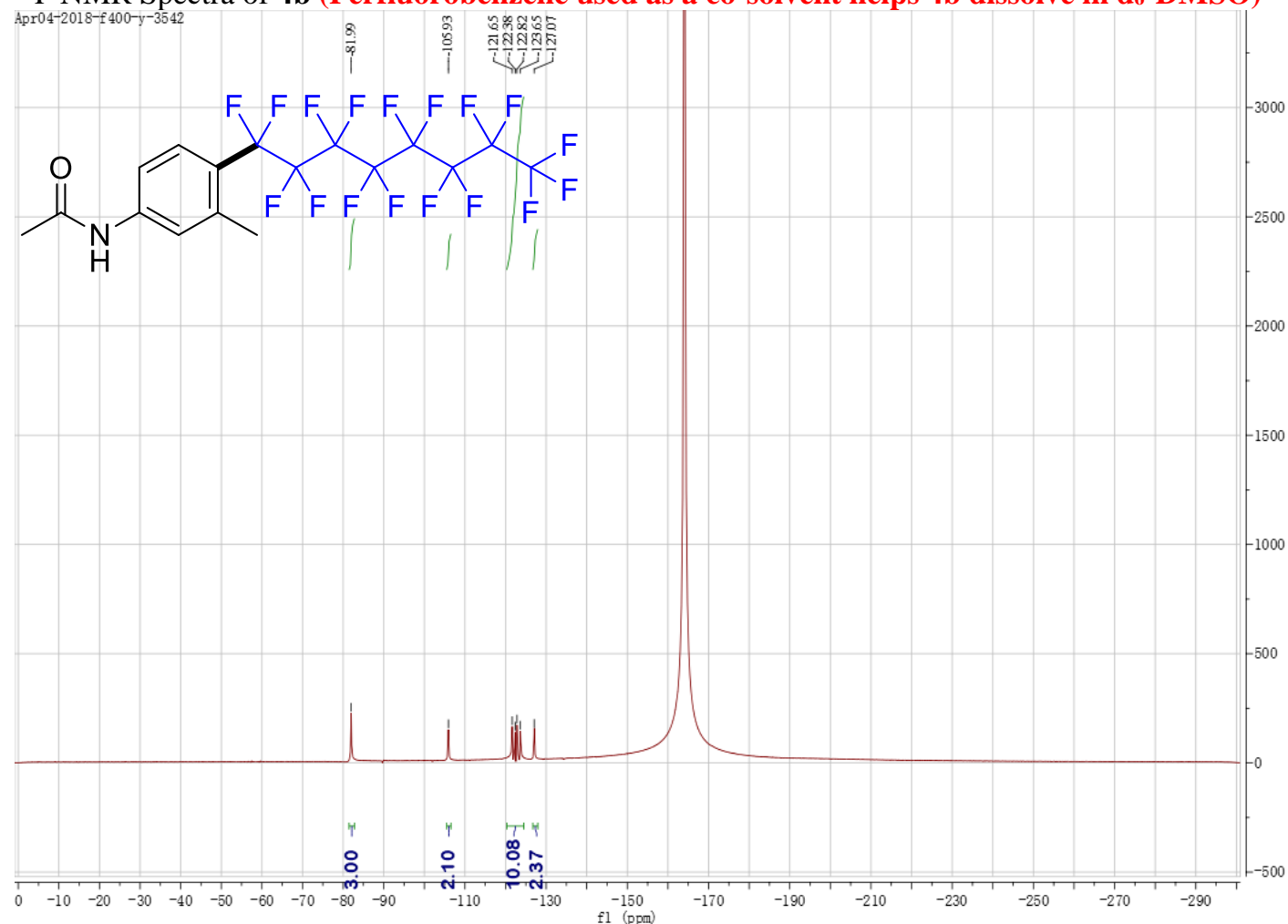

${ }^{13} \mathrm{C}$ NMR Spectra of $\mathbf{4 b}$ (Perfluorobenzene used as a co-solvent helps $4 \mathrm{~b}$ dissolve in $\mathrm{d}_{6}$-DMSO)

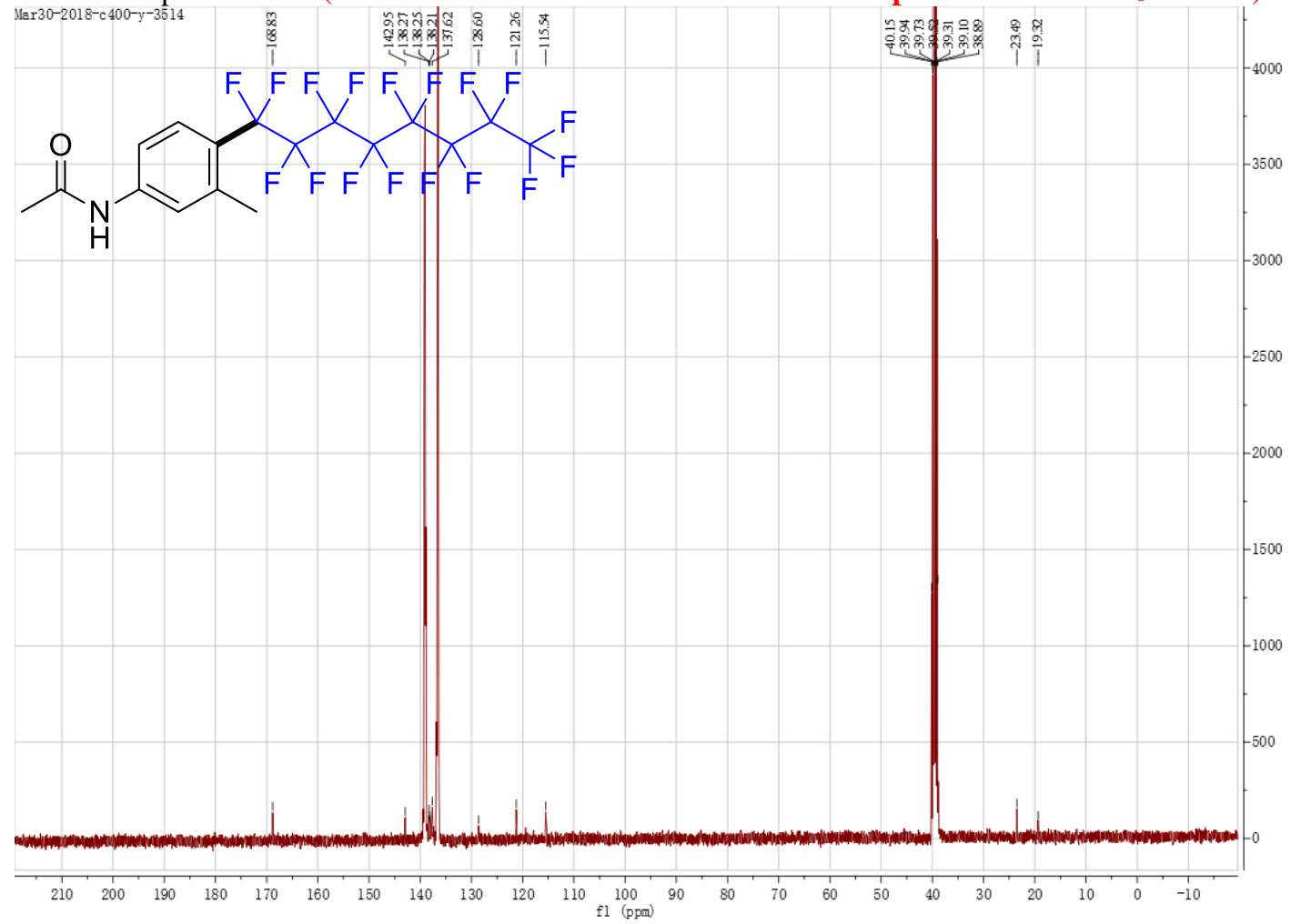


${ }^{1} \mathrm{H}$ NMR Spectra of $4 \mathrm{c}$ (Perfluorobenzene used as a co-solvent helps $4 \mathrm{c}$ dissolve in d6-DMSO)

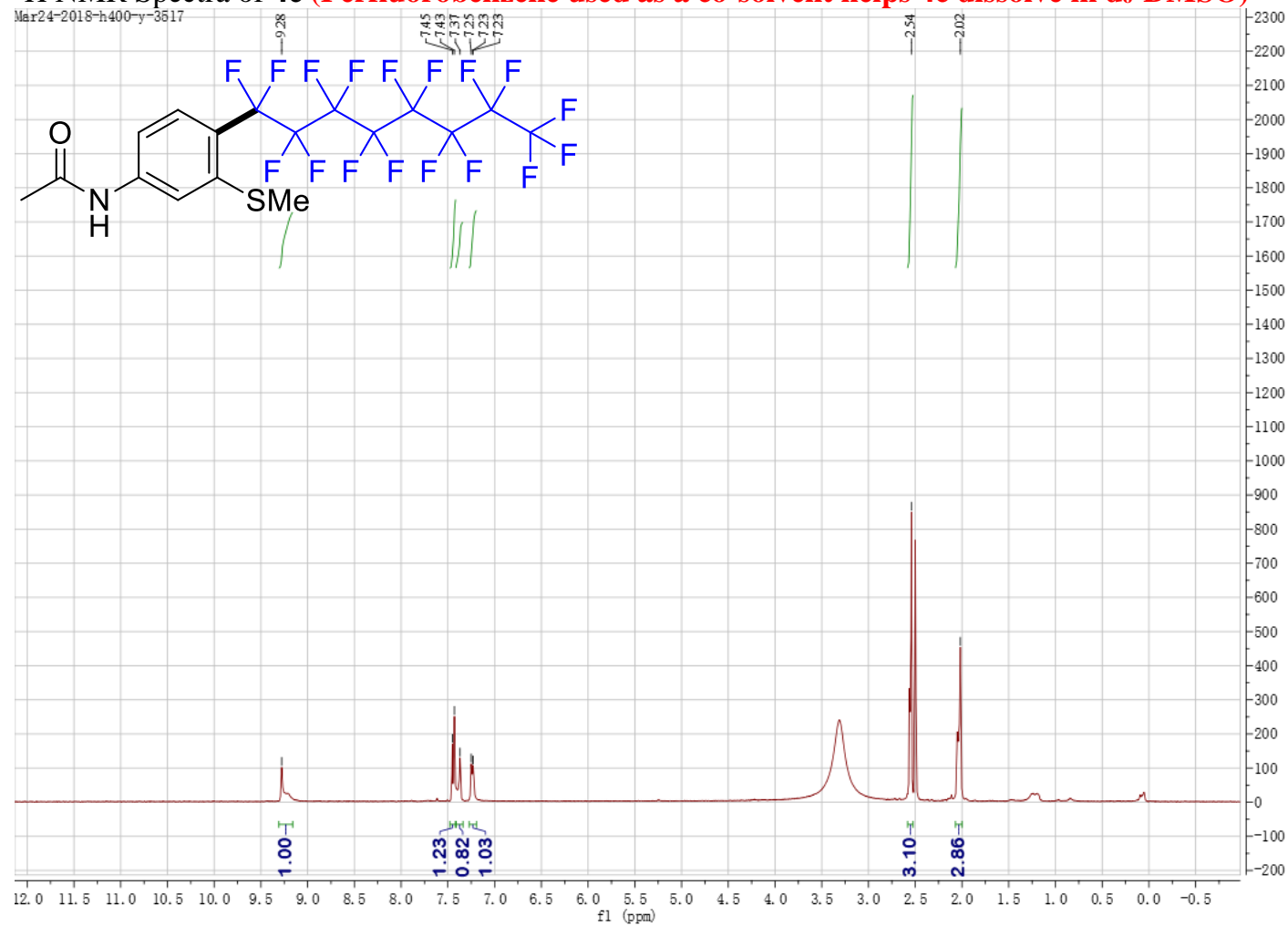

${ }^{19} \mathrm{~F}$ NMR Spectra of $\mathbf{4 c}$ (Perfluorobenzene used as a co-solvent helps $4 \mathrm{c}$ dissolve in $\mathrm{d}_{6}$-DMSO)

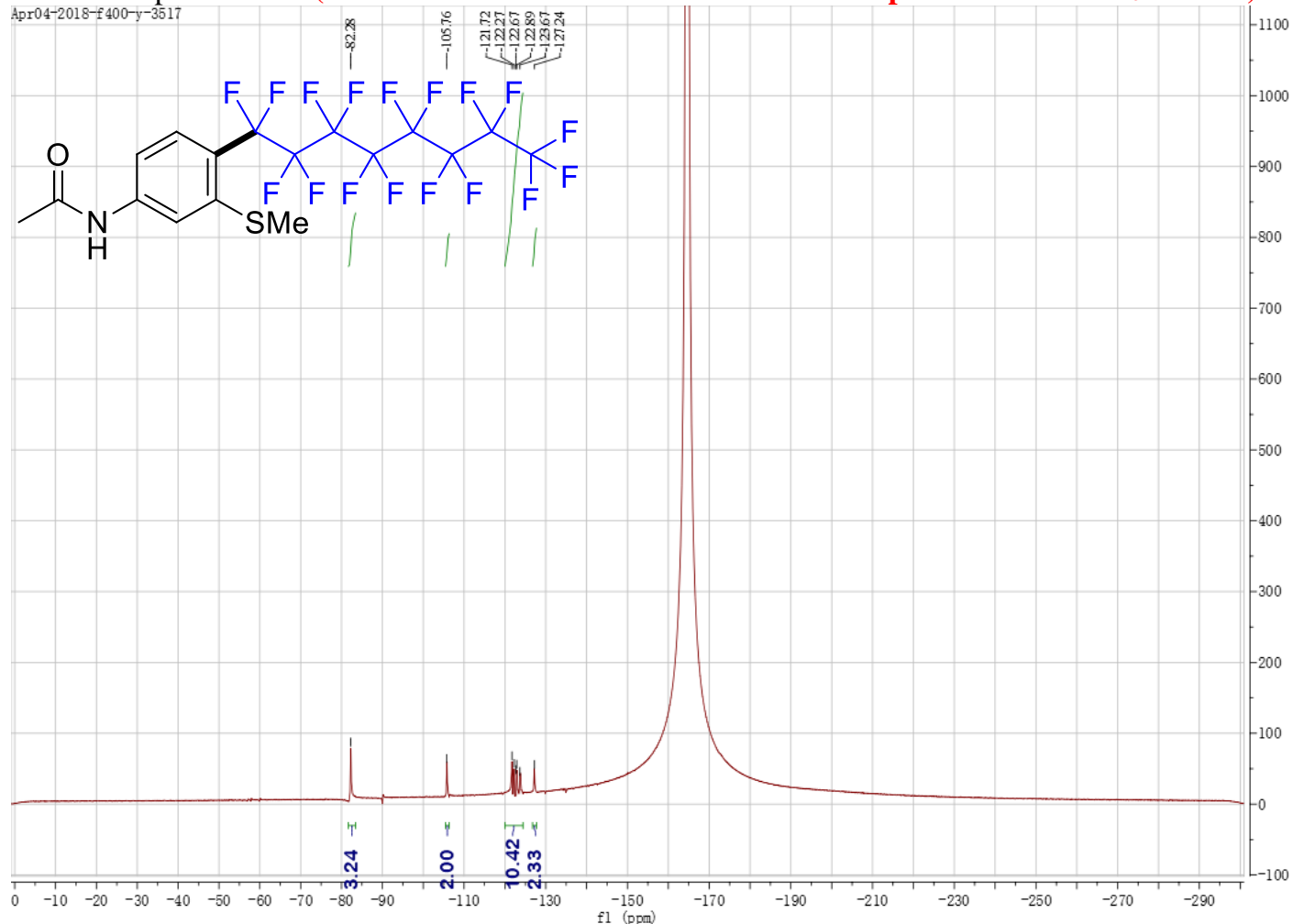


${ }^{13} \mathrm{C}$ NMR Spectra of $4 \mathrm{c}$ (Perfluorobenzene used as a co-solvent helps 4c dissolve in $\mathrm{d}_{6}$-DMSO)

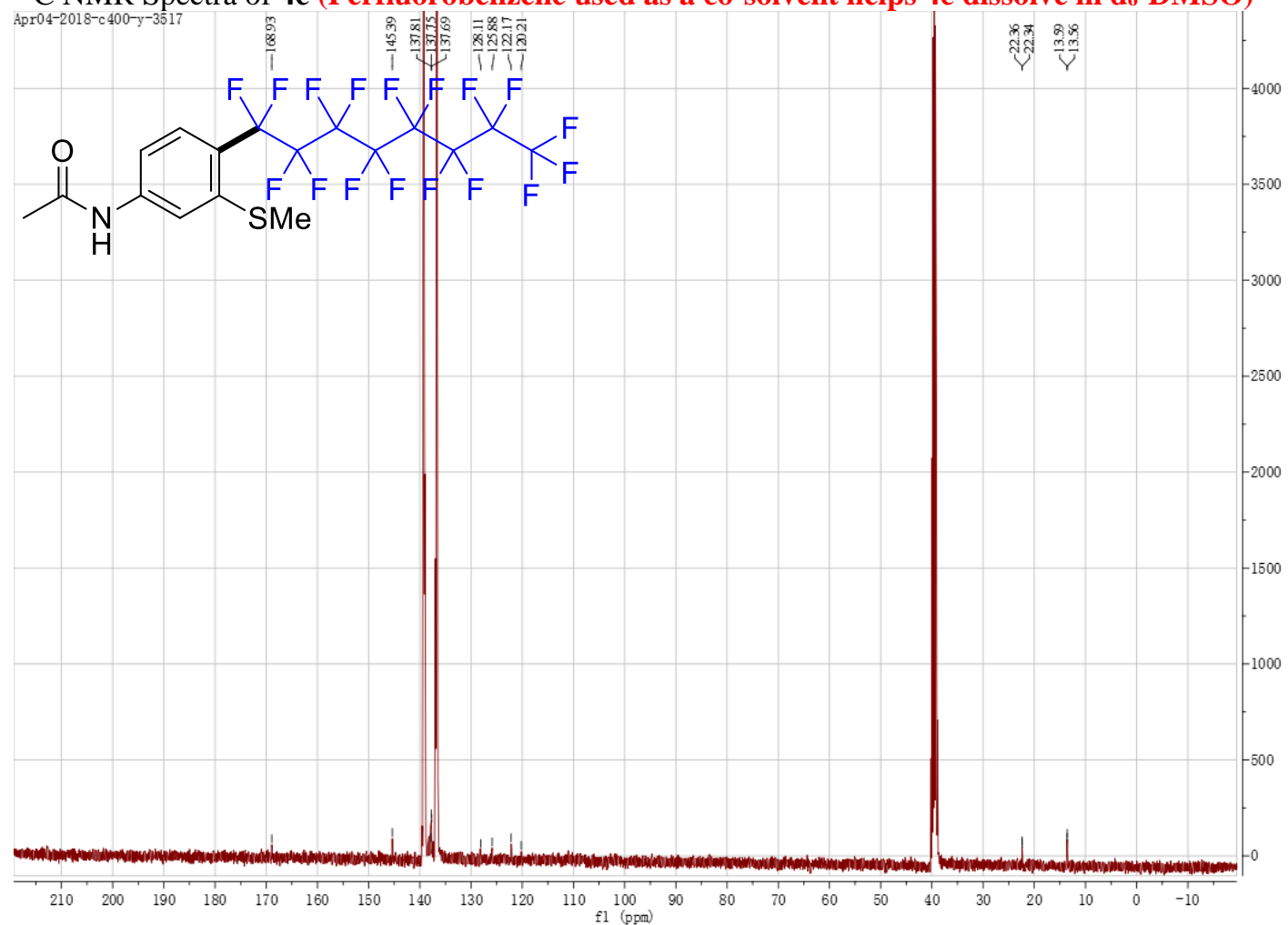

${ }^{1} \mathrm{H}$ NMR, ${ }^{19} \mathrm{~F}$ NMR \& ${ }^{13} \mathrm{C}$ NMR Spectra of $4 \mathbf{d}$

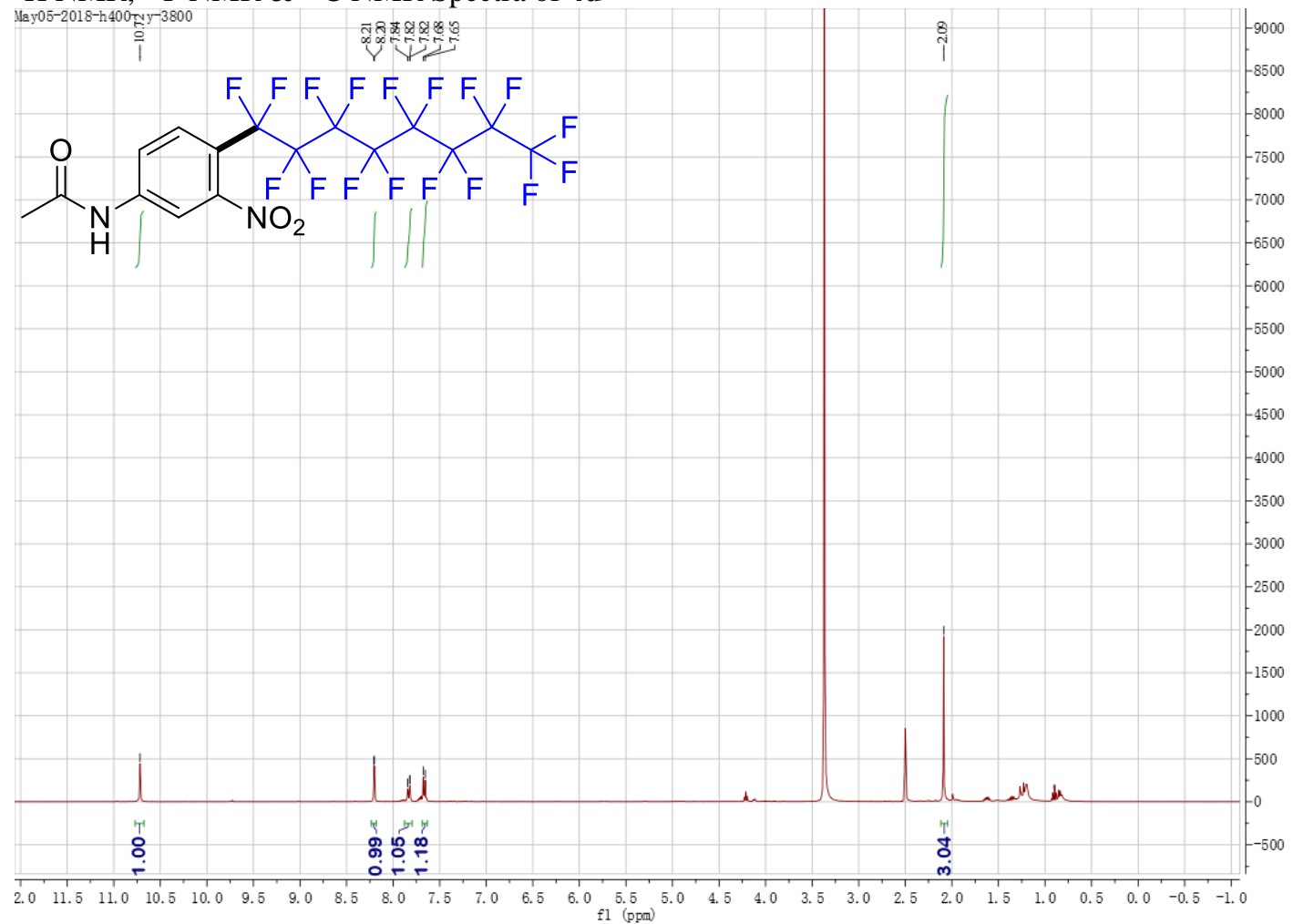


${ }^{19} \mathrm{~F}$ NMR Spectra of $\mathbf{4 d}$

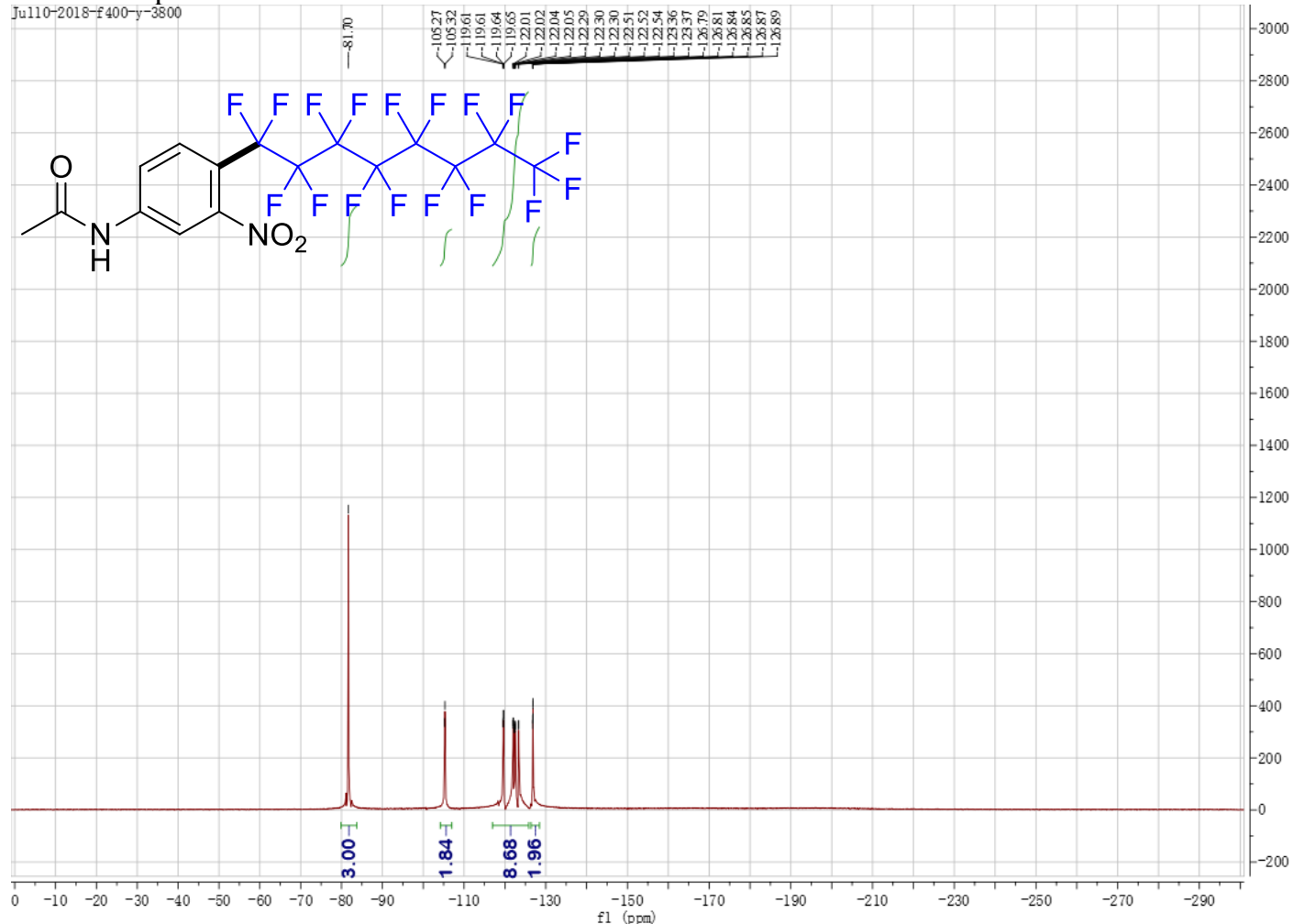

${ }^{13} \mathrm{C}$ NMR Spectra of $\mathbf{4 d}$

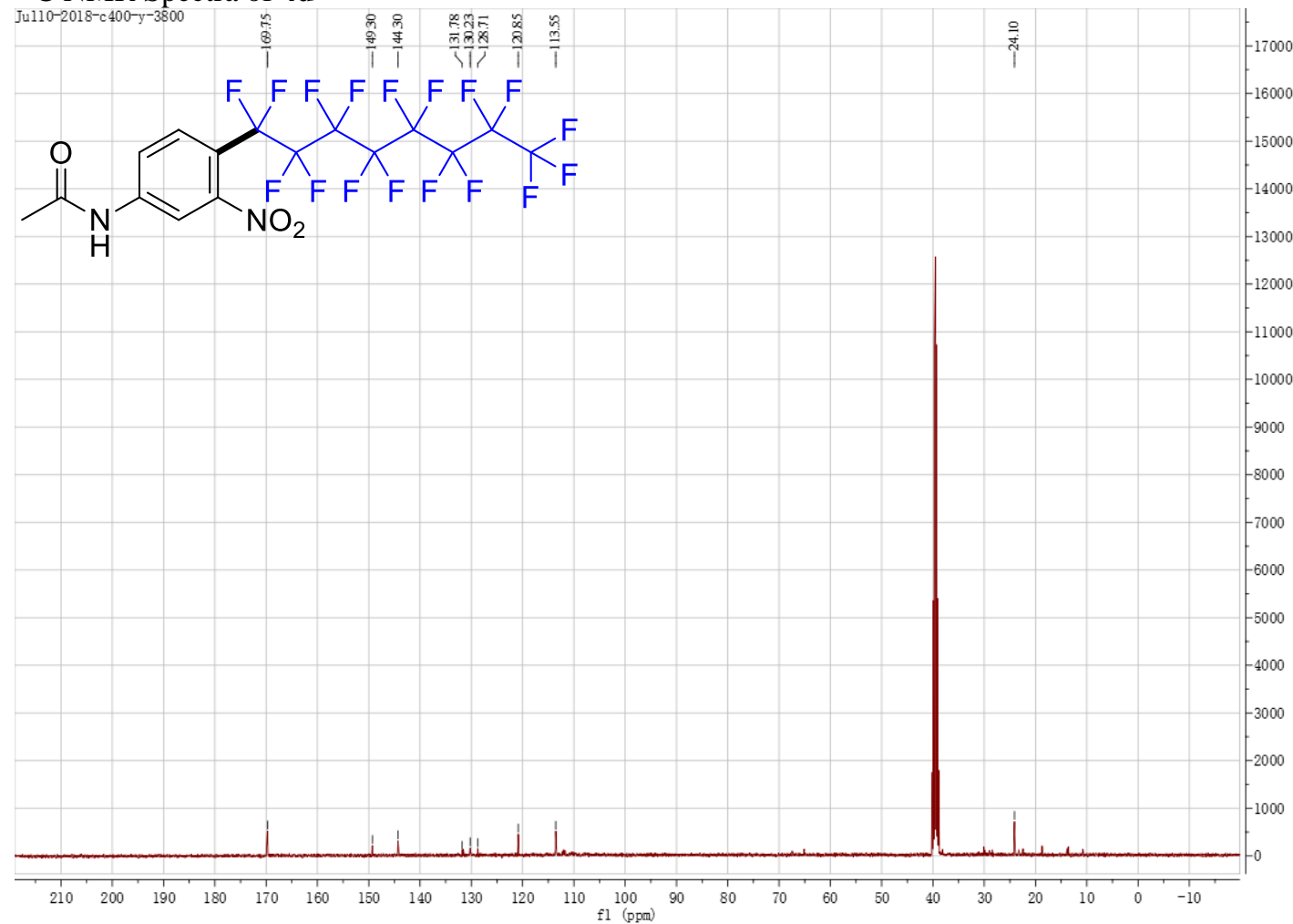


${ }^{1} \mathrm{H}$ NMR, ${ }^{19} \mathrm{~F}$ NMR \& ${ }^{13} \mathrm{C}$ NMR Spectra of $4 \mathbf{e}$ (Perfluorobenzene used as a co-solvent helps $4 \mathrm{e}$ dissolve in $\mathrm{d}_{6}-\mathrm{DMSO}$ )

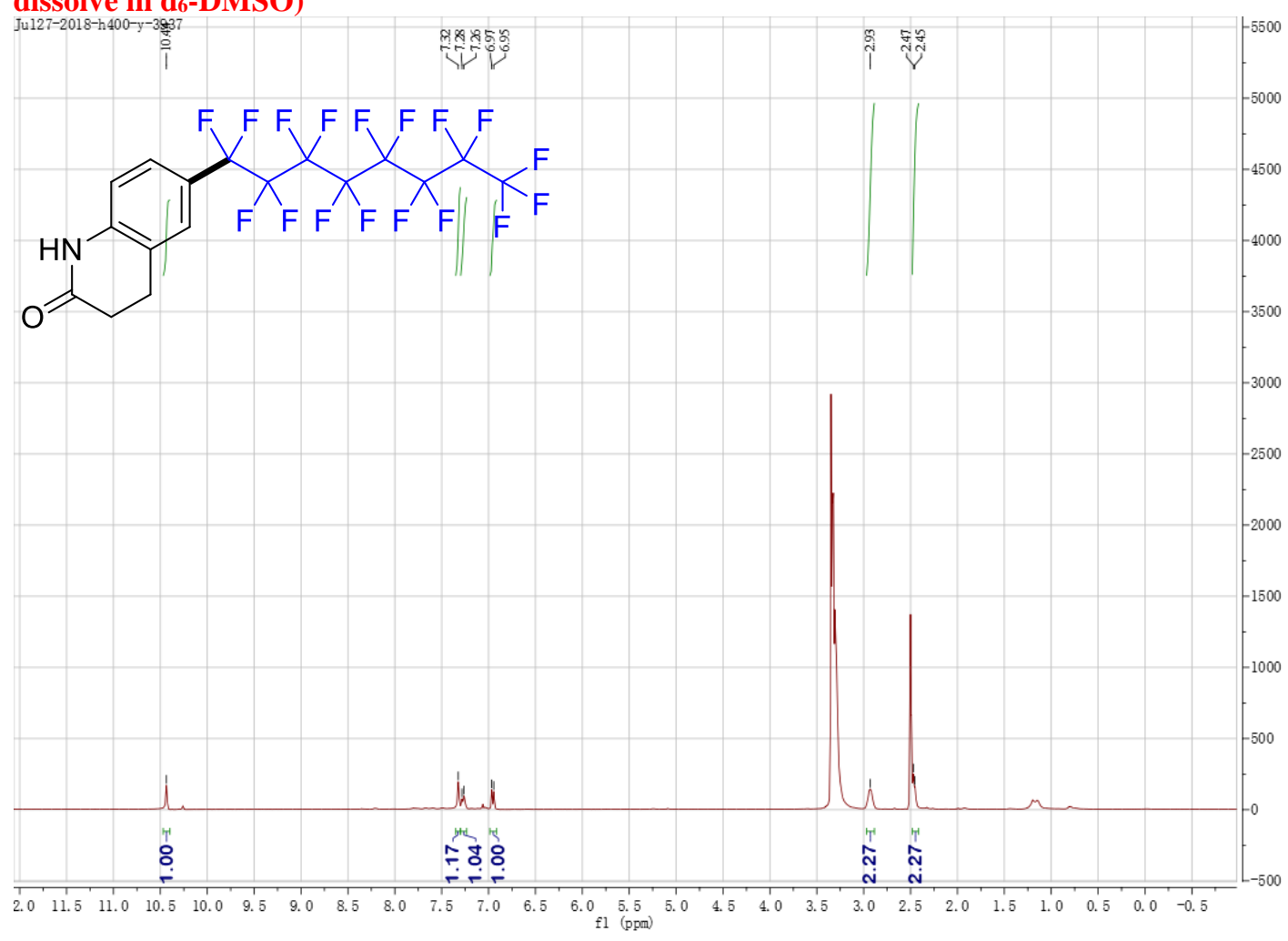

${ }^{19} \mathrm{~F}$ NMR Spectra of $\mathbf{4 e}$ (Perfluorobenzene used as a co-solvent helps $4 \mathrm{e}$ dissolve in $\mathrm{d}_{6}$-DMSO)

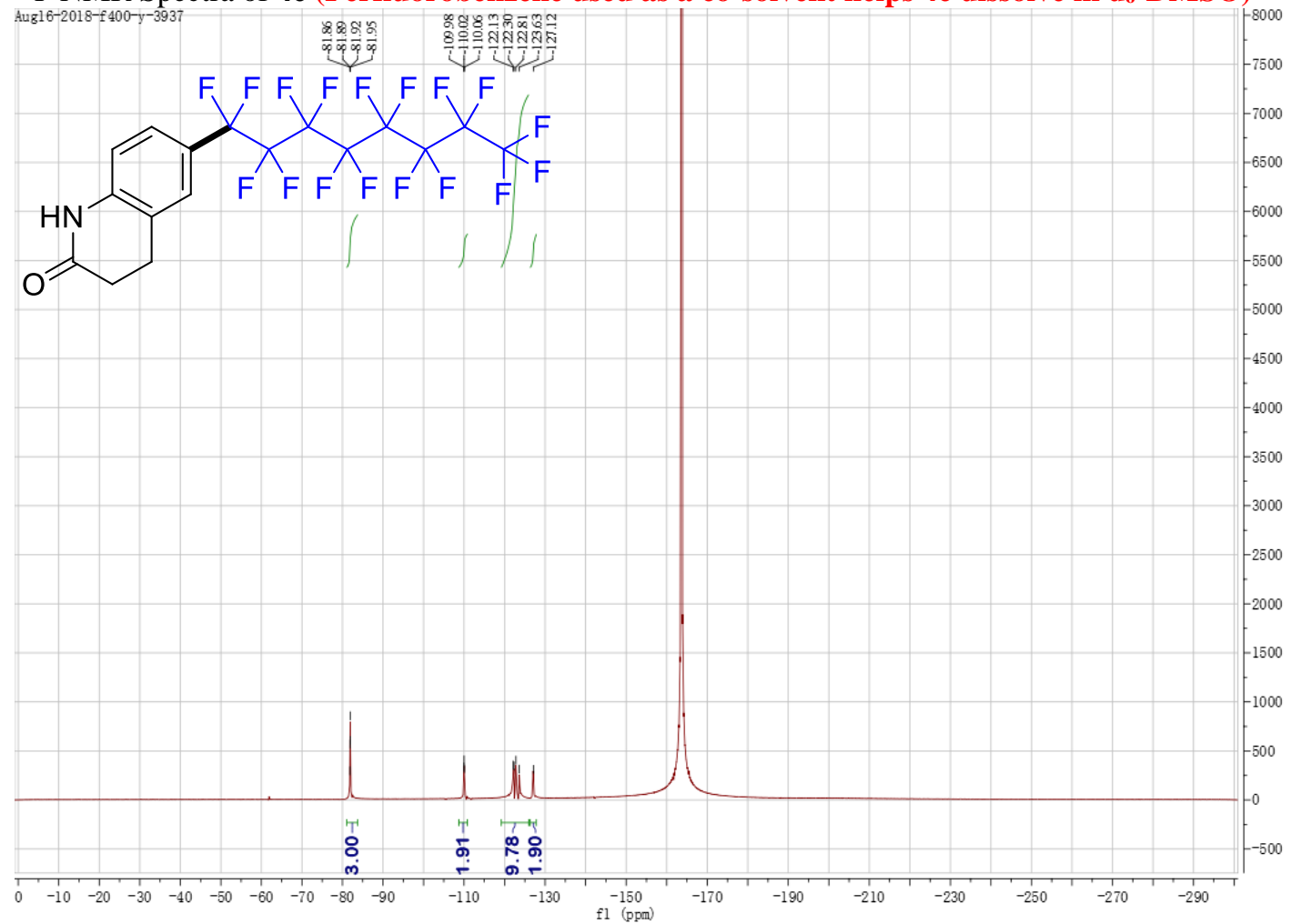


${ }^{13} \mathrm{C}$ NMR Spectra of $4 \mathbf{e}$ (Perfluorobenzene used as a co-solvent helps 4e dissolve in $\mathrm{d}_{6}$-DMSO)

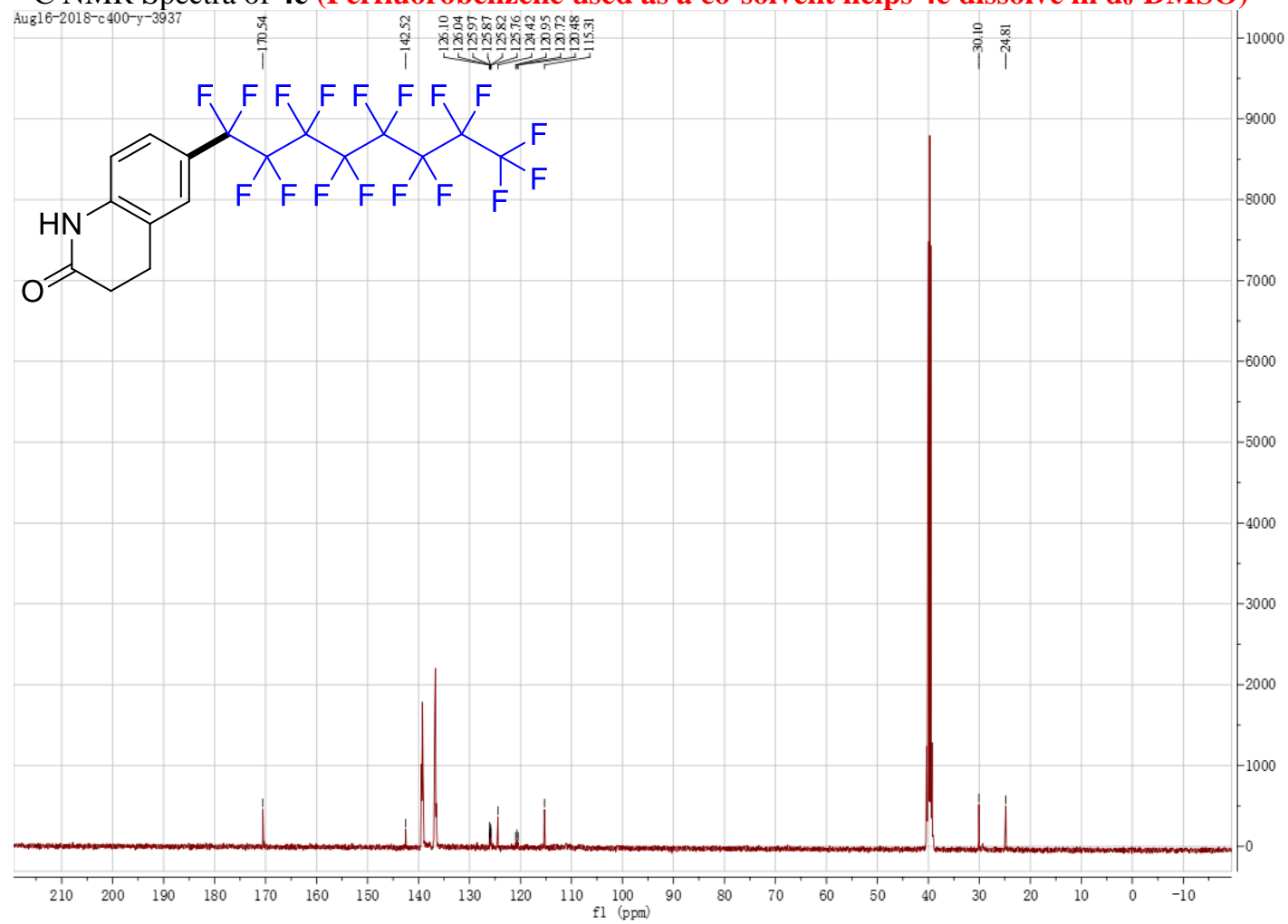

${ }^{1} \mathrm{H}$ NMR Spectra of $\mathbf{4 f}$ (Perfluorobenzene used as a co-solvent helps $\mathbf{4 f}$ dissolve in $\mathbf{d}_{6}$-DMSO)

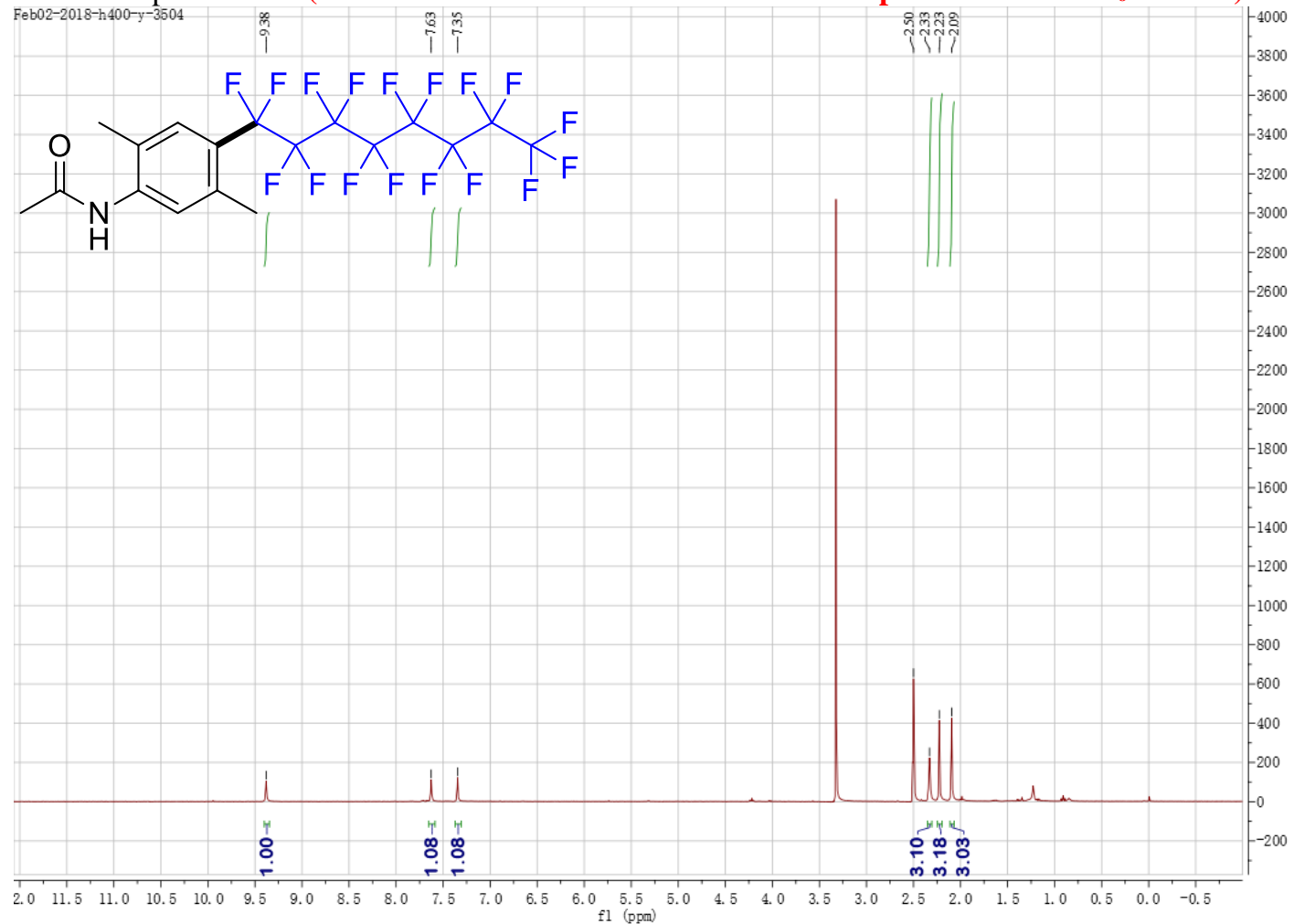


${ }^{19} \mathrm{~F}$ NMR Spectra of $4 \mathrm{f}$ (Perfluorobenzene used as a co-solvent helps $4 \mathrm{f}$ dissolve in $\mathrm{d}_{6}$-DMSO) Feb02-2018-400-y-3504 $\quad$ o

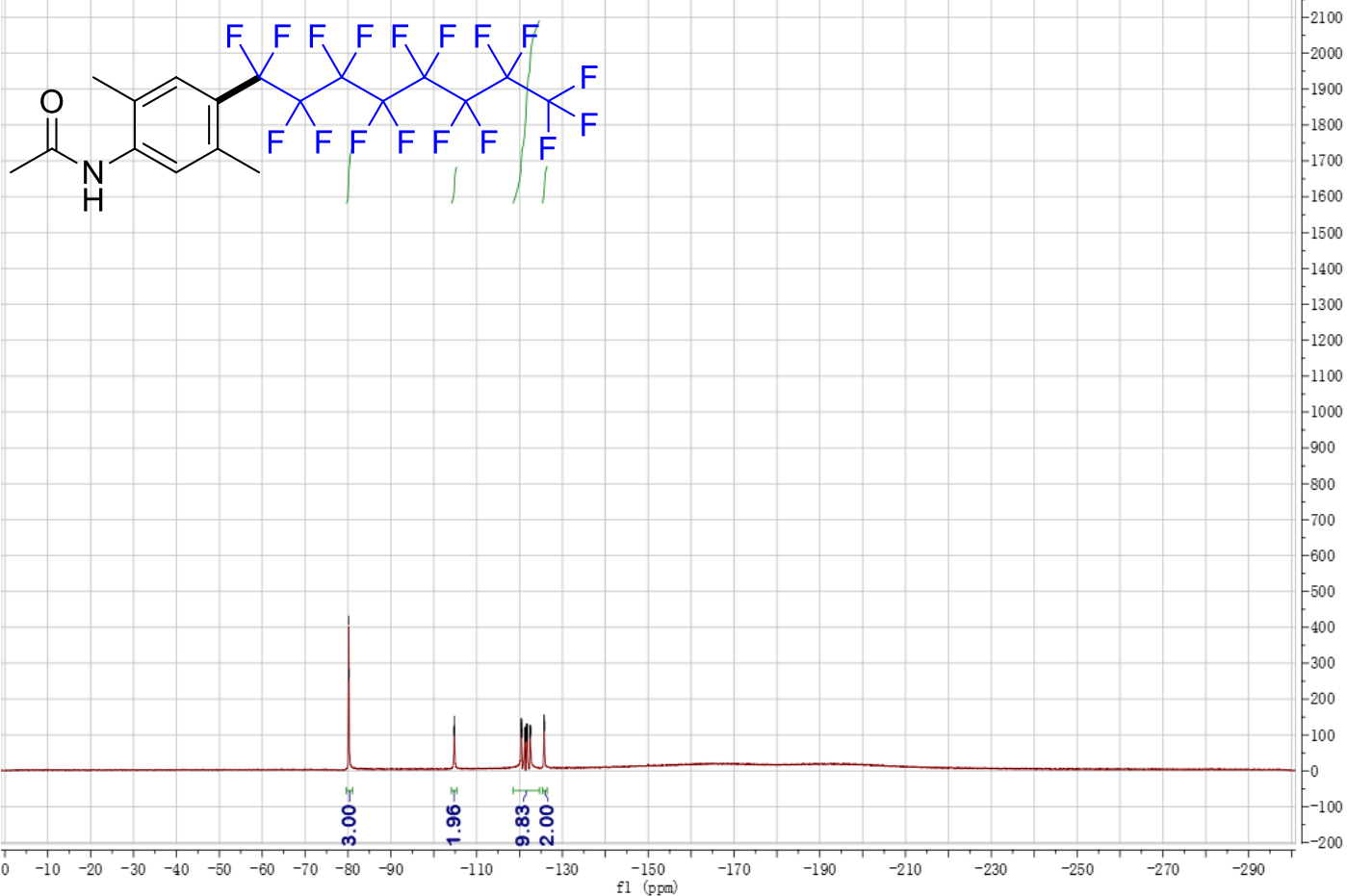

${ }^{13} \mathrm{C}$ NMR Spectra of $4 \mathbf{f}$ (Perfluorobenzene used as a co-solvent helps $4 \mathbf{f}$ dissolve in $\mathrm{d}_{6}$-DMSO)

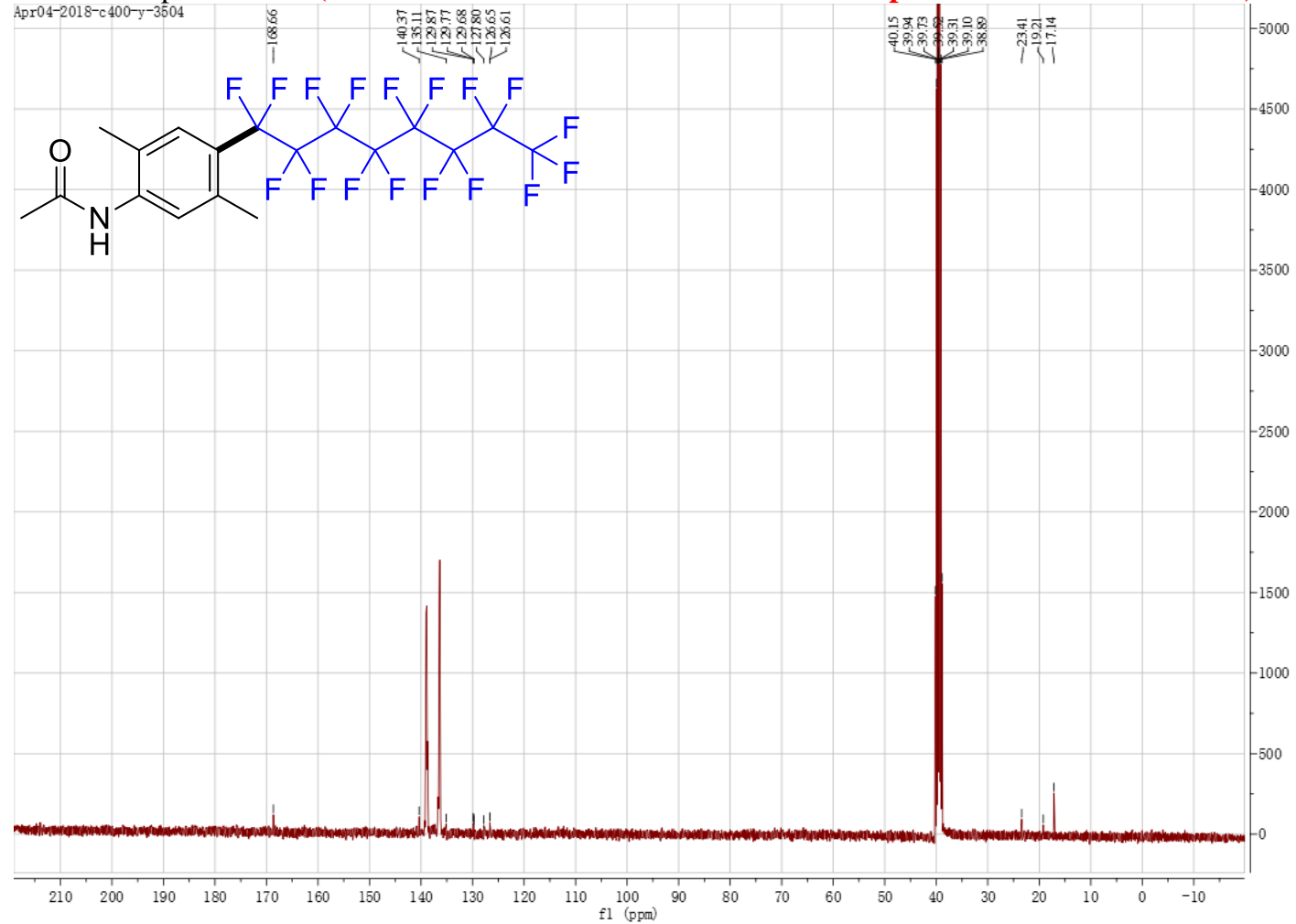


${ }^{1} \mathrm{H}$ NMR Spectra of $\mathbf{4 g}$

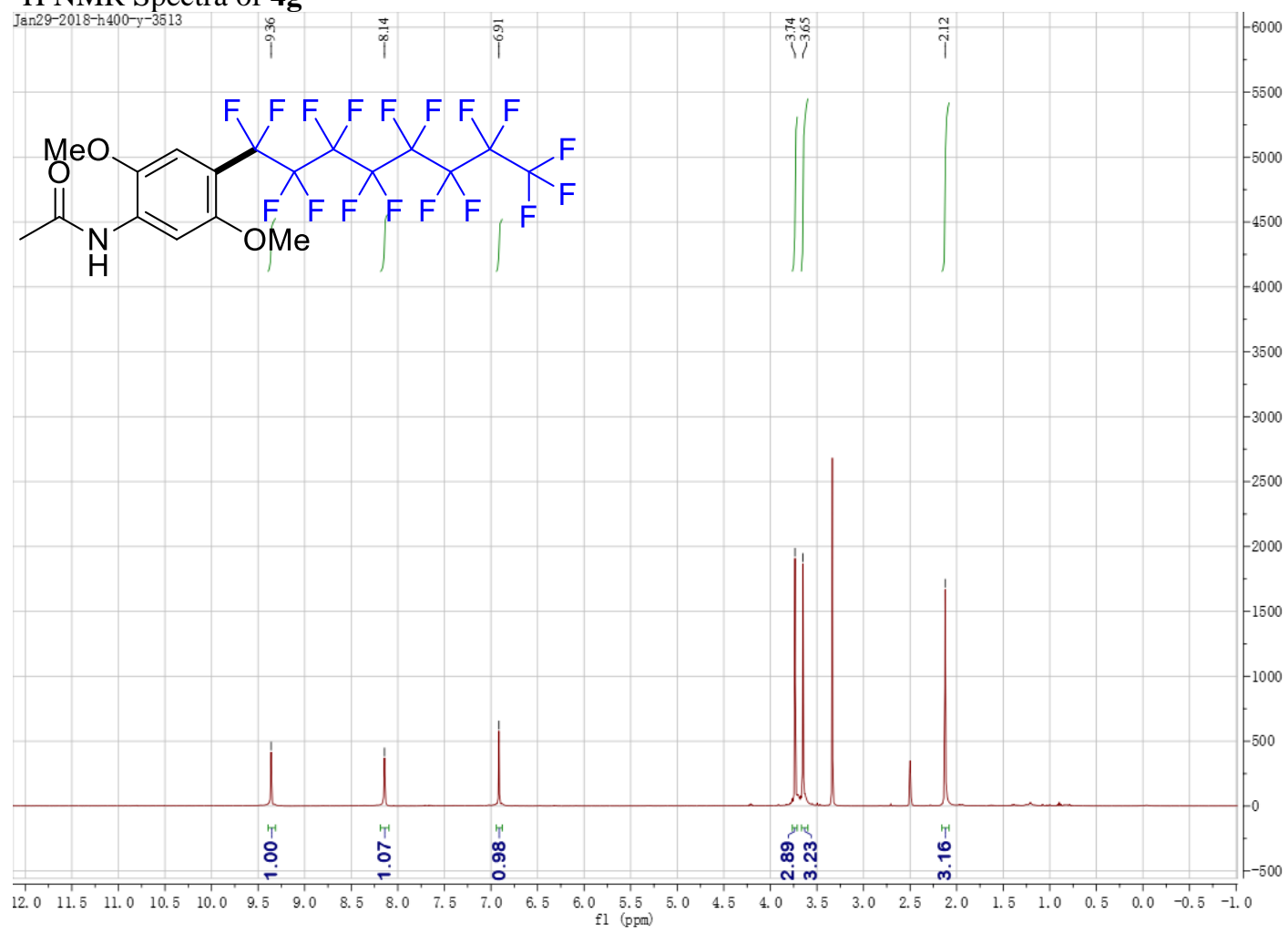

${ }^{19} \mathrm{~F}$ NMR Spectra of $\mathbf{4 g}$

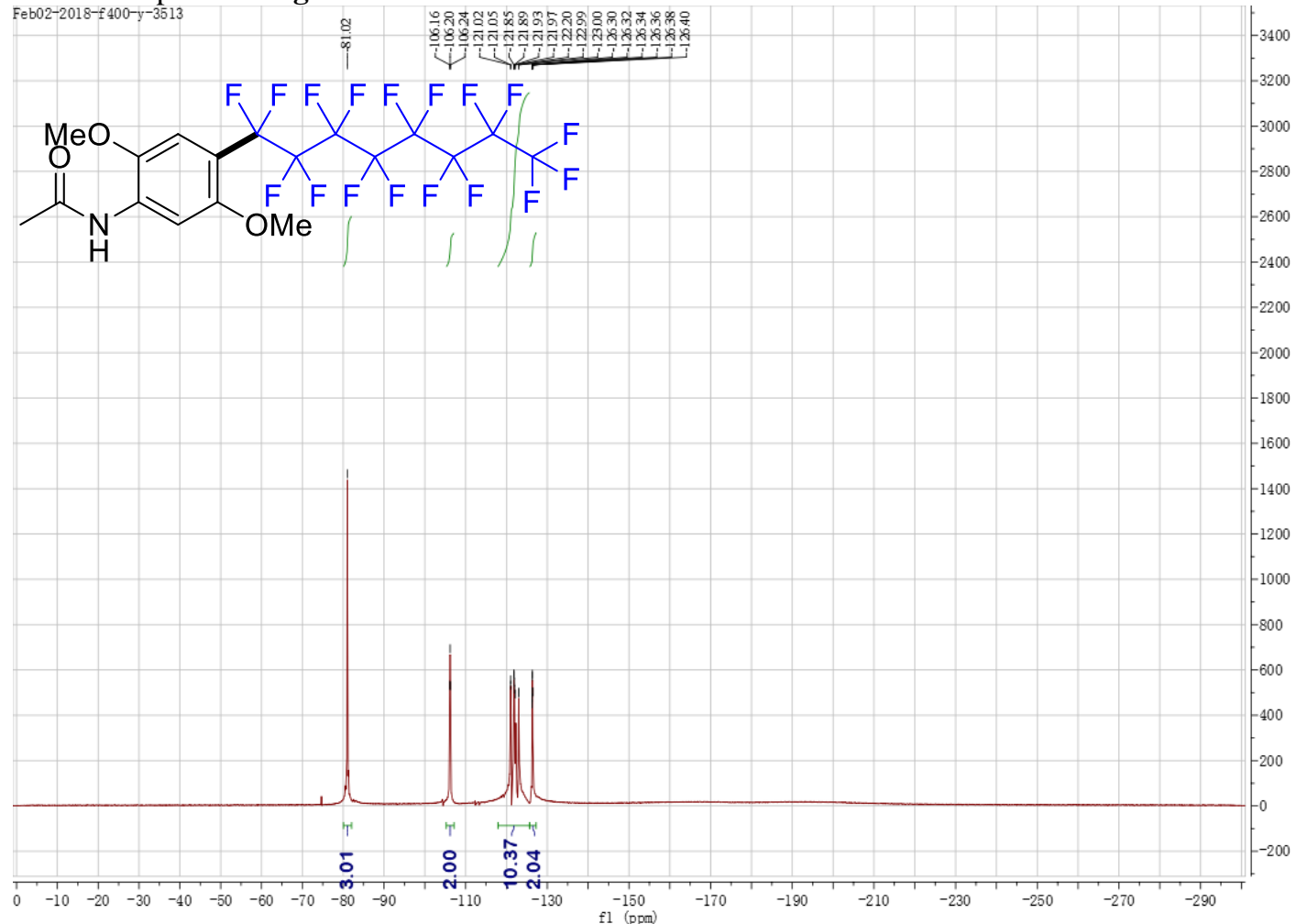


${ }^{13} \mathrm{C}$ NMR $\mathrm{NM}$ Spe-2018-c400-y-35513

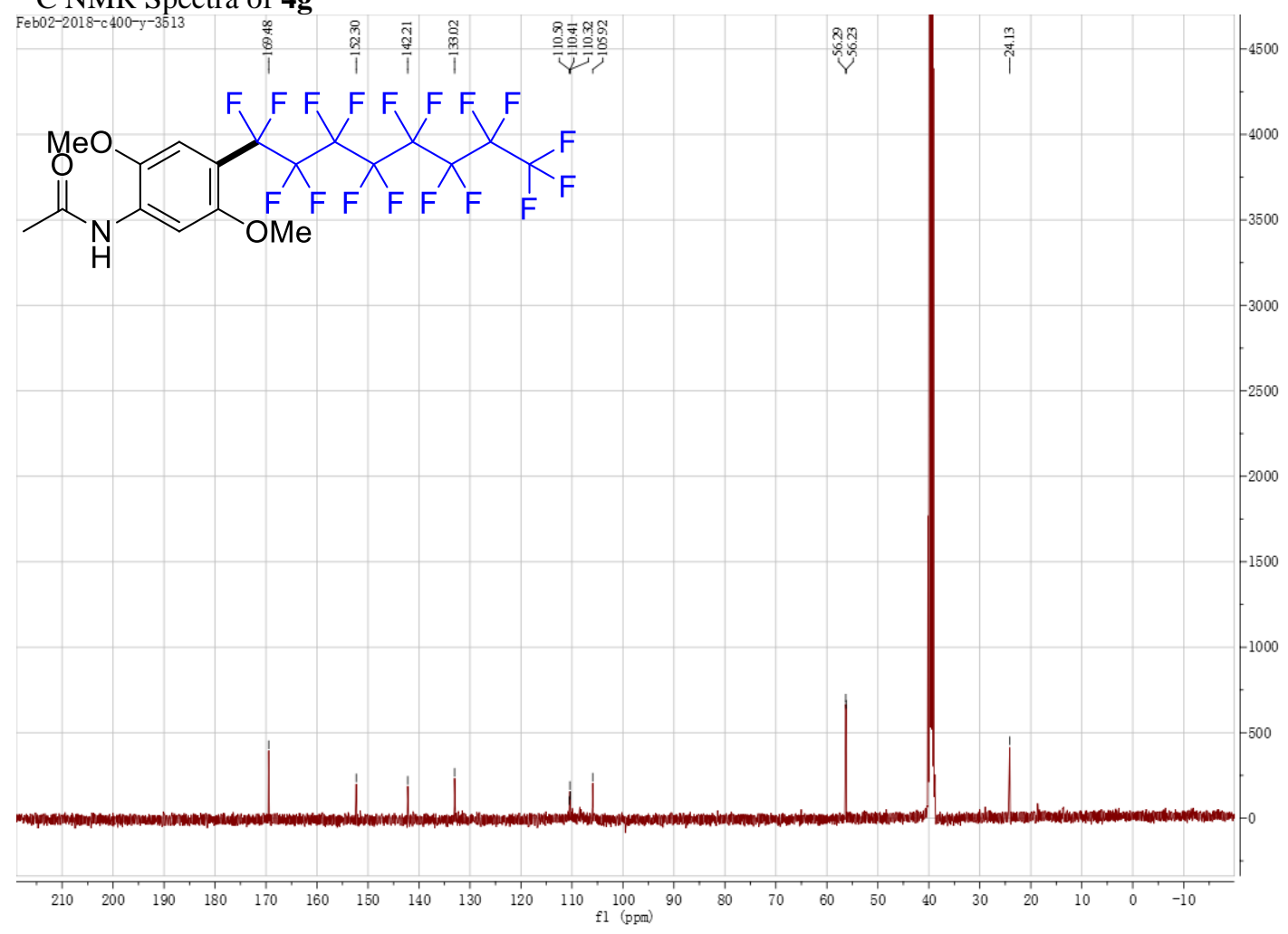

${ }^{1}$ H NMR Spectra of $\mathbf{4 h}$

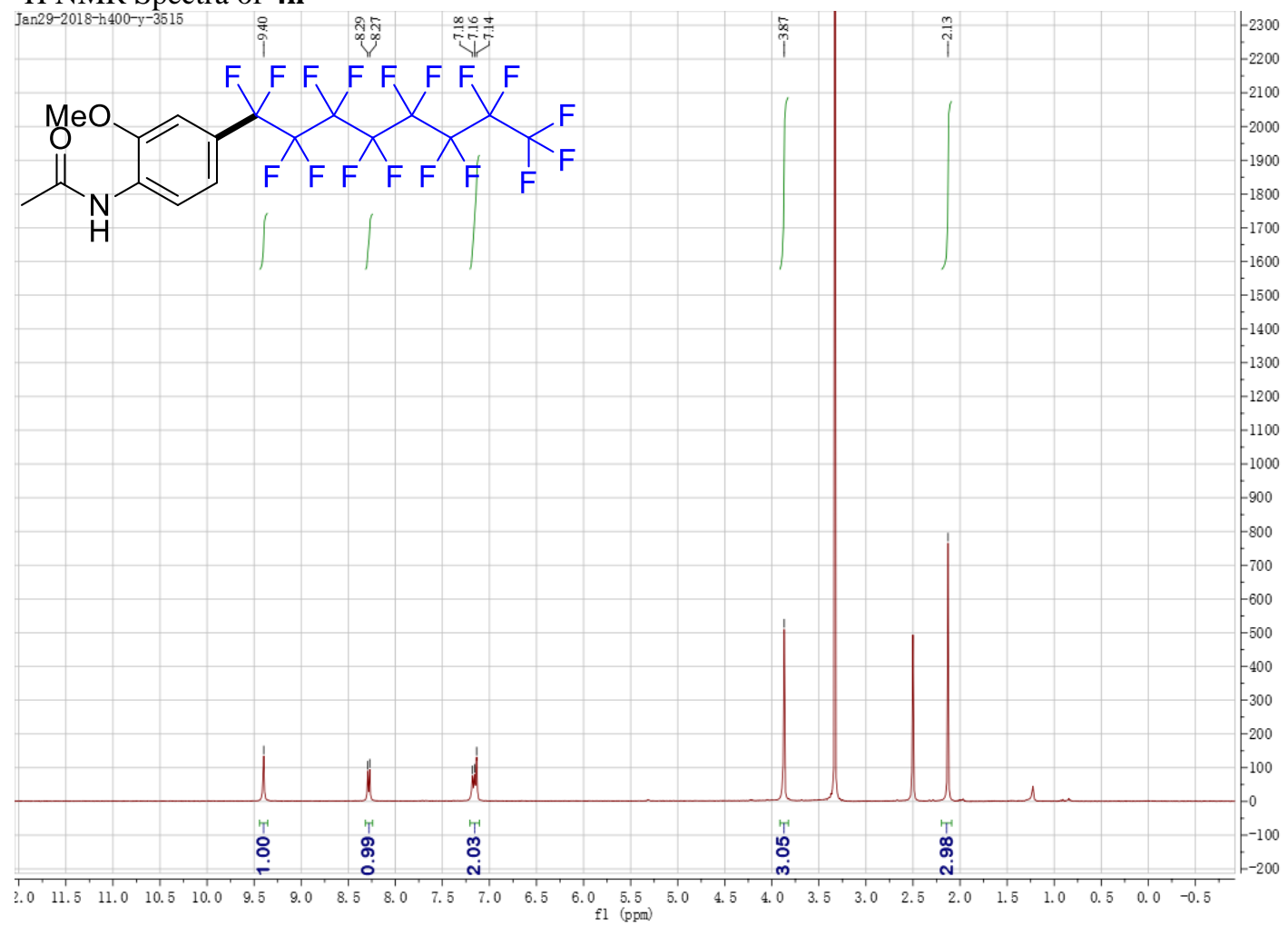


${ }^{19} \mathrm{~F}$ NMR Spectra of $\mathbf{4 h}$

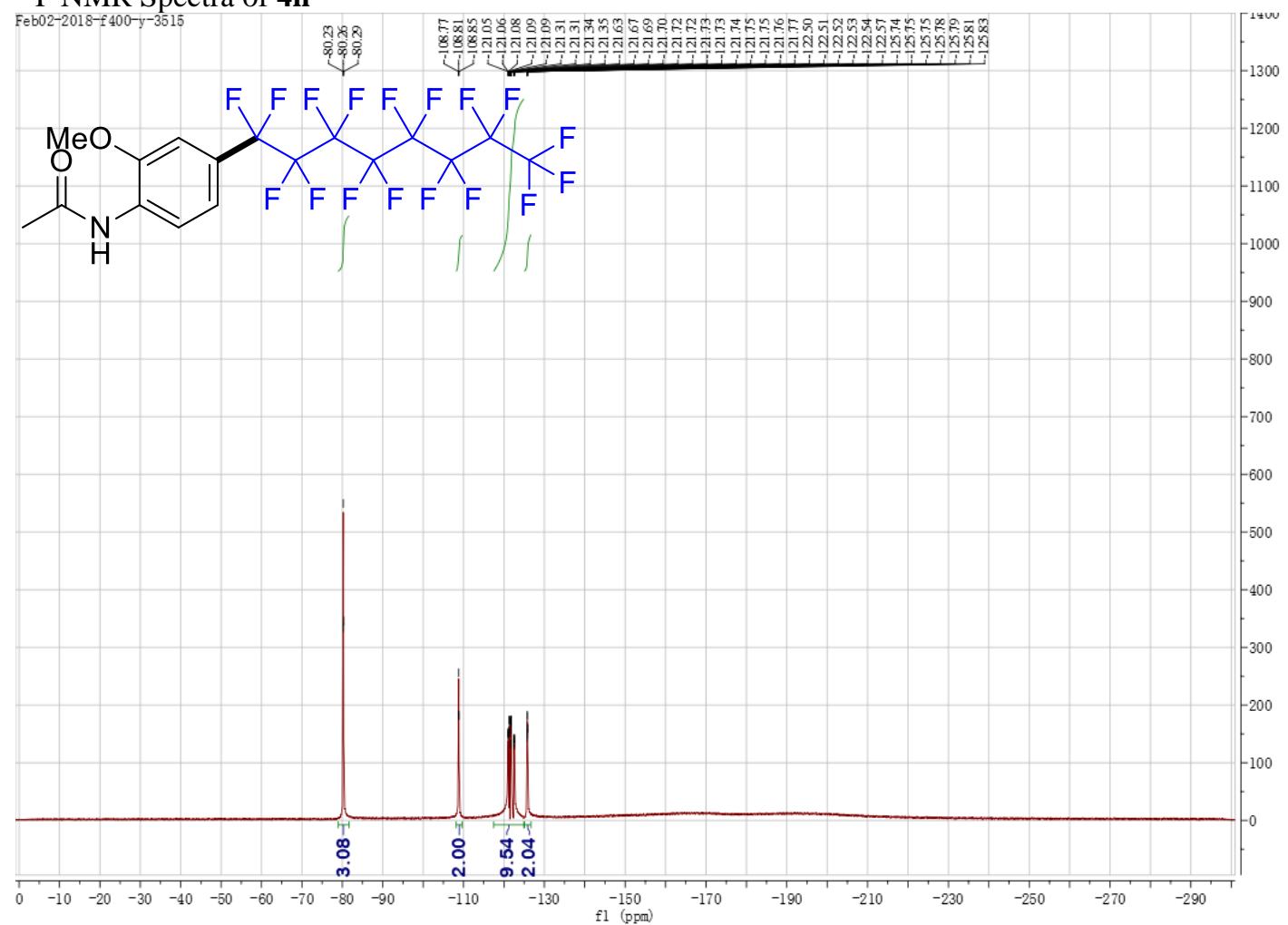

${ }^{13} \mathrm{C}$ NMR Spectra of $\mathbf{4 h}$

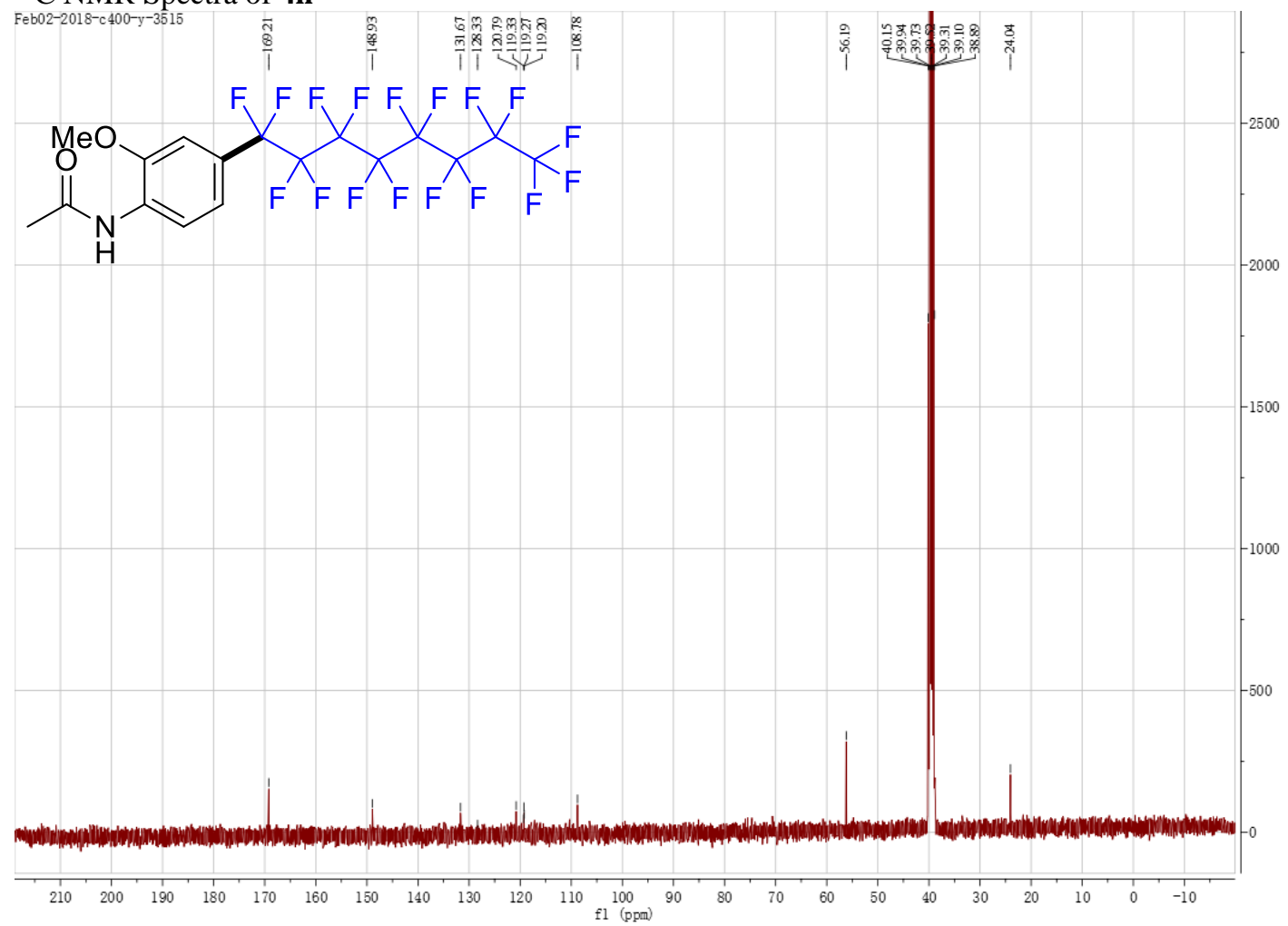


${ }^{1} \mathrm{H}$ NMR, ${ }^{19} \mathrm{~F}$ NMR \& ${ }^{13} \mathrm{C}$ NMR Spectra of $4 \mathbf{i}$ (Perfluorobenzene used as a co-solvent helps $4 \mathbf{i}$ dissolve in $\mathrm{d}_{6}$-DMSO)

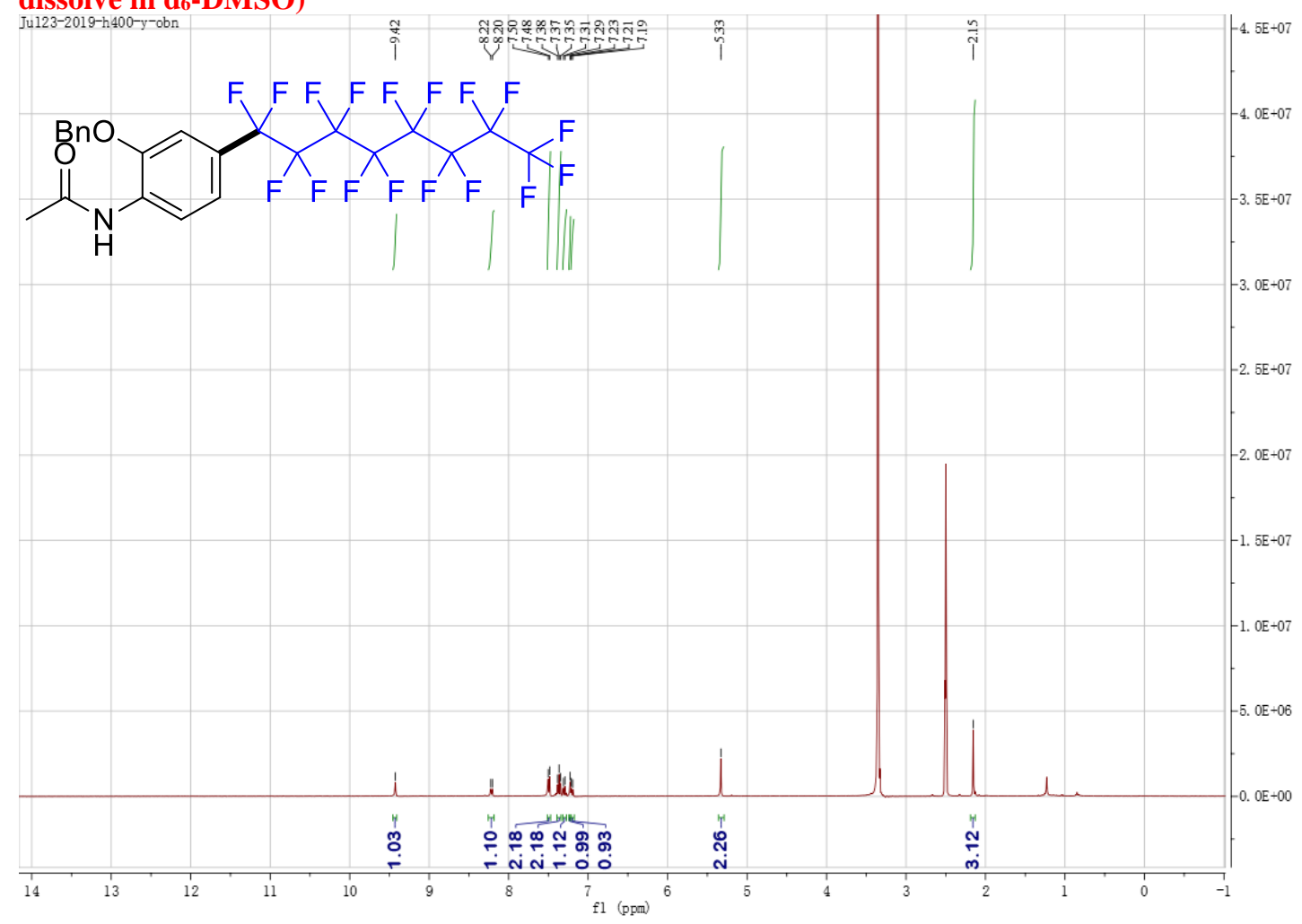

${ }^{19} \mathrm{~F}$ NMR Spectra of $4 \mathbf{i}$ (Perfluorobenzene used as a co-solvent helps $4 \mathbf{i}$ dissolve in $\mathbf{d}_{6}$-DMSO)

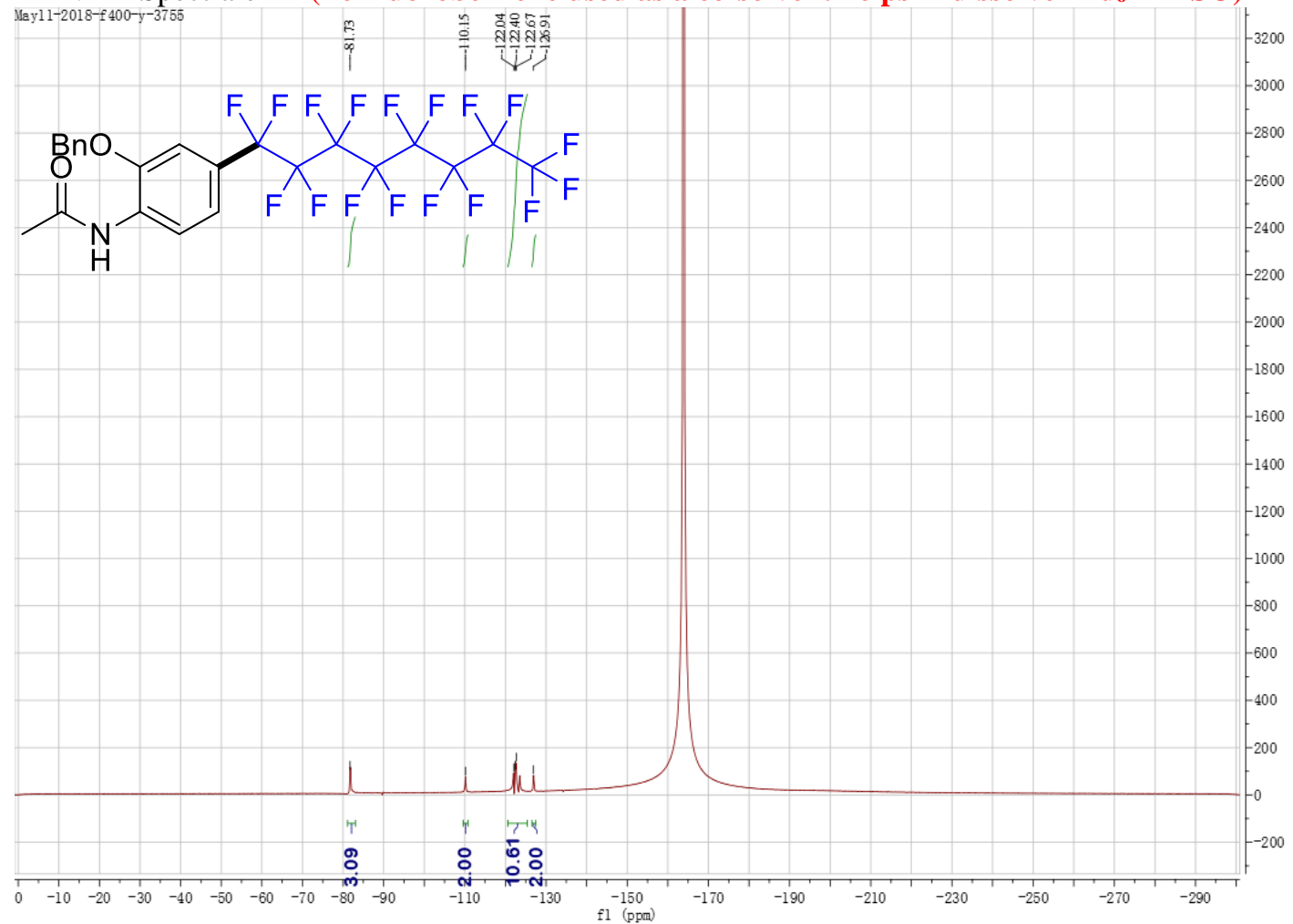


${ }^{13} \mathrm{C}$ NMR Spectra of 4i (Perfluorobenzene used as a co-solvent helps 4i dissolve in $\mathrm{d}_{6}$-DMSO)

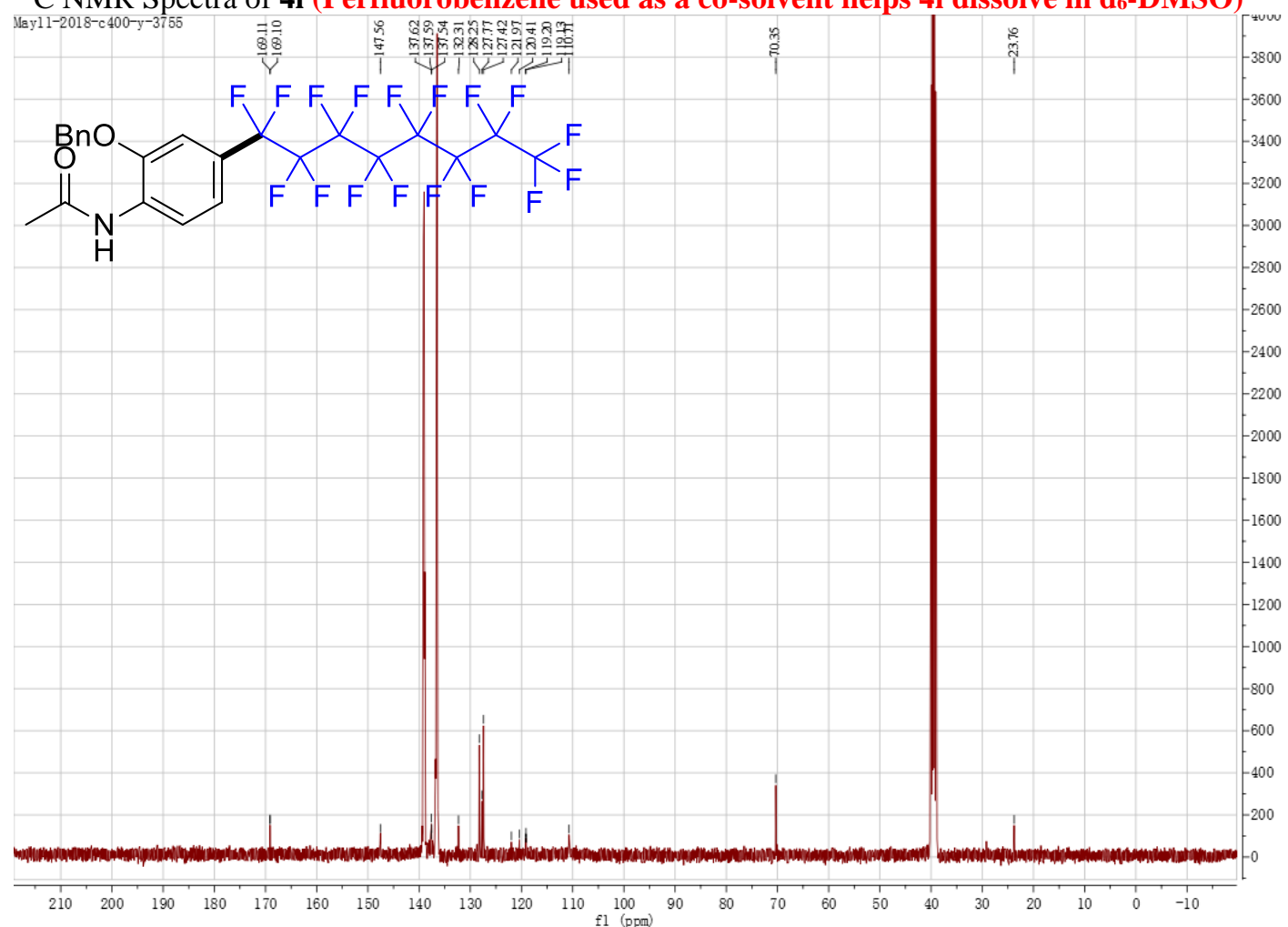

${ }^{1} \mathrm{H}$ NMR Spectra of $\mathbf{4 j}$ (Perfluorobenzene used as a co-solvent helps $4 \mathbf{j}$ dissolve in $\mathbf{d}_{6}$-DMSO)

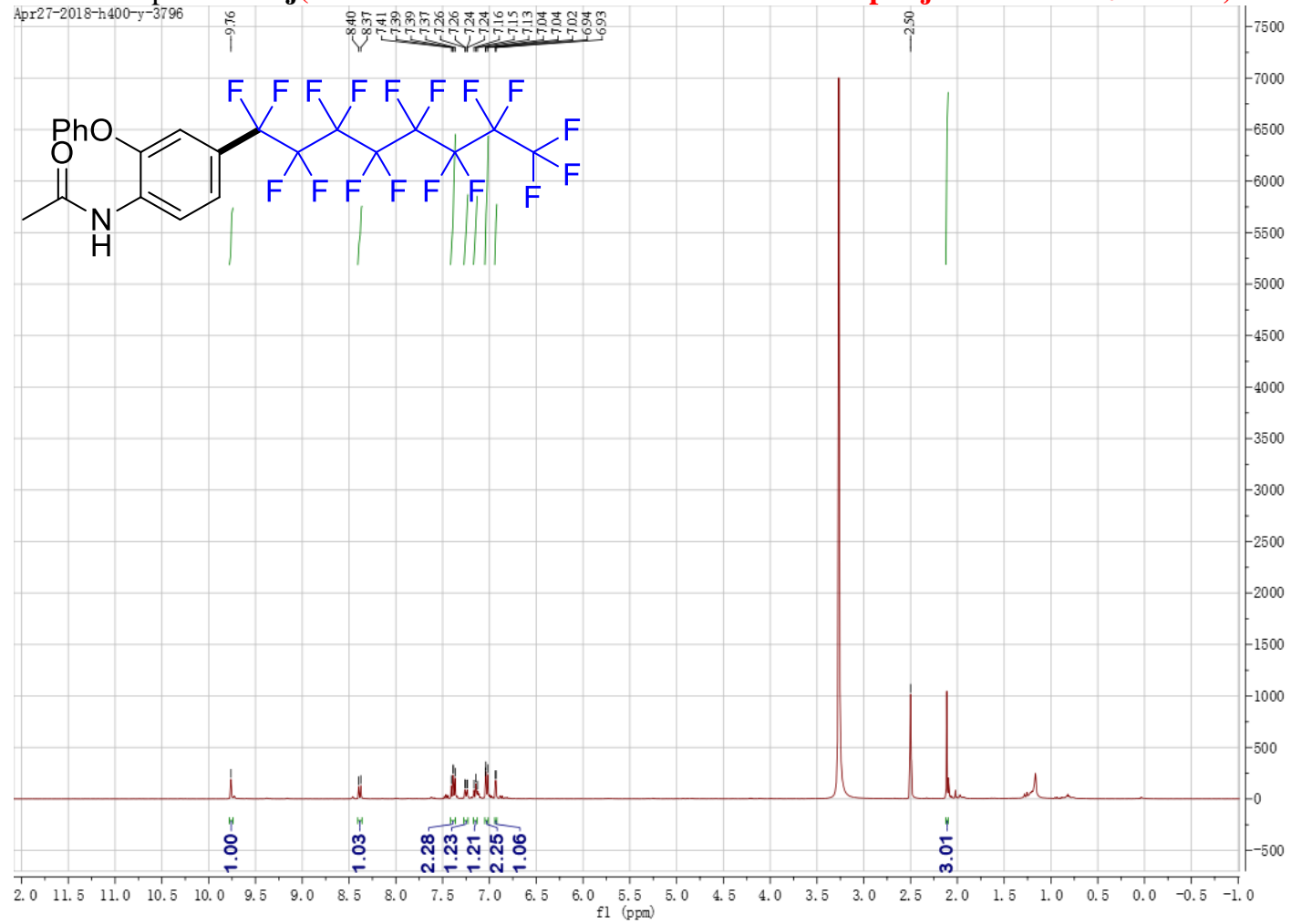


${ }^{19} \mathrm{~F}$ NMR Spectra of $\mathbf{4 j}$ (Perfluorobenzene used as a co-solvent helps $\mathbf{4 j}$ dissolve in $\mathbf{d}_{6}$-DMSO)

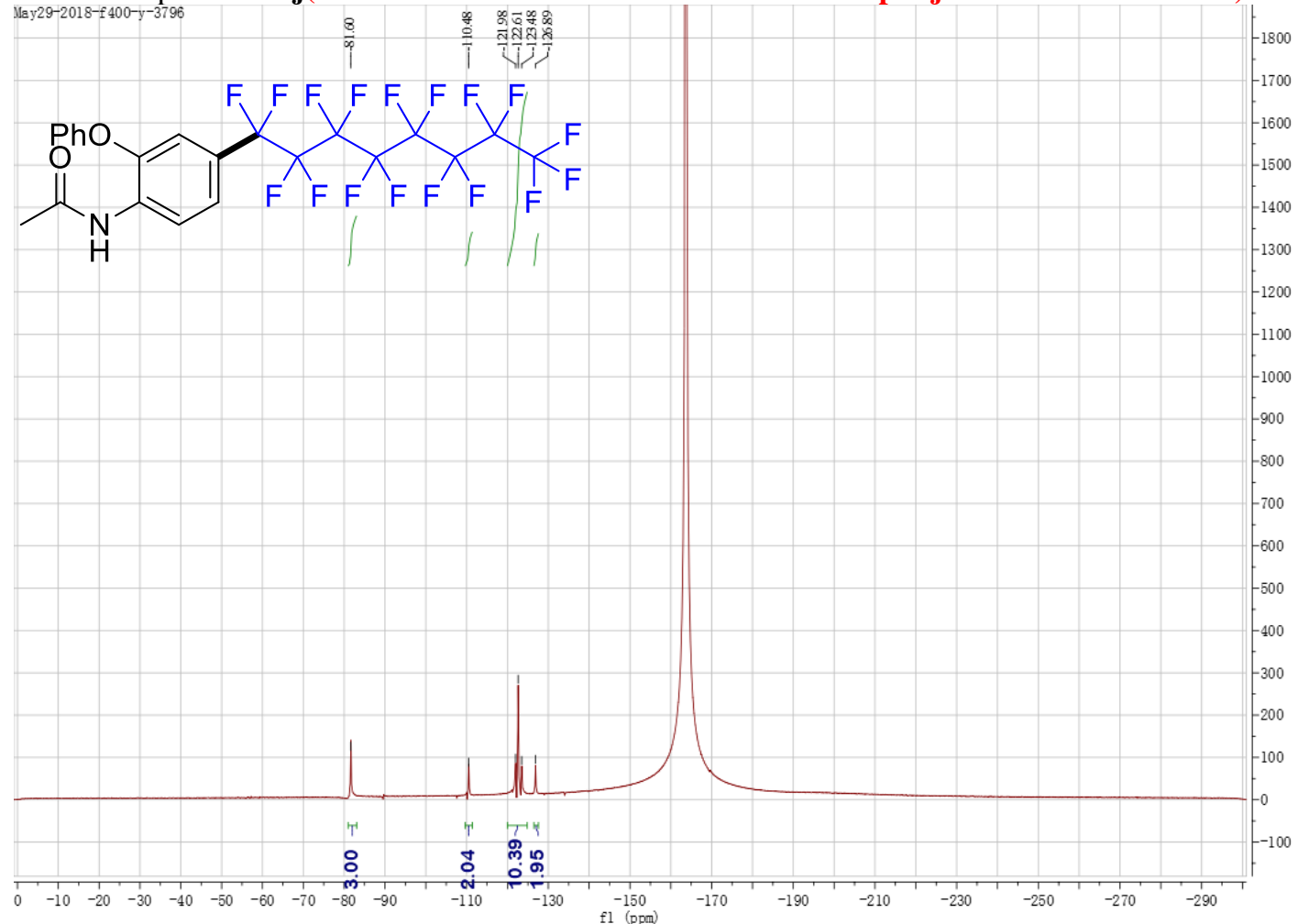

${ }^{13} \mathrm{C}$ NMR Spectra of $\mathbf{4 j}$ (Perfluorobenzene used as a co-solvent helps $4 \mathbf{j}$ dissolve in $\mathbf{d}_{6}$-DMSO)

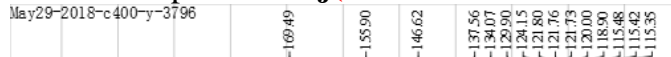<smiles>CC(=O)Nc1ccc(C(F)(F)C(F)(F)C(F)(F)C(F)(F)C(F)(F)C(F)(F)C(F)(F)C(F)(F)C(F)(F)F)cc1O</smiles>

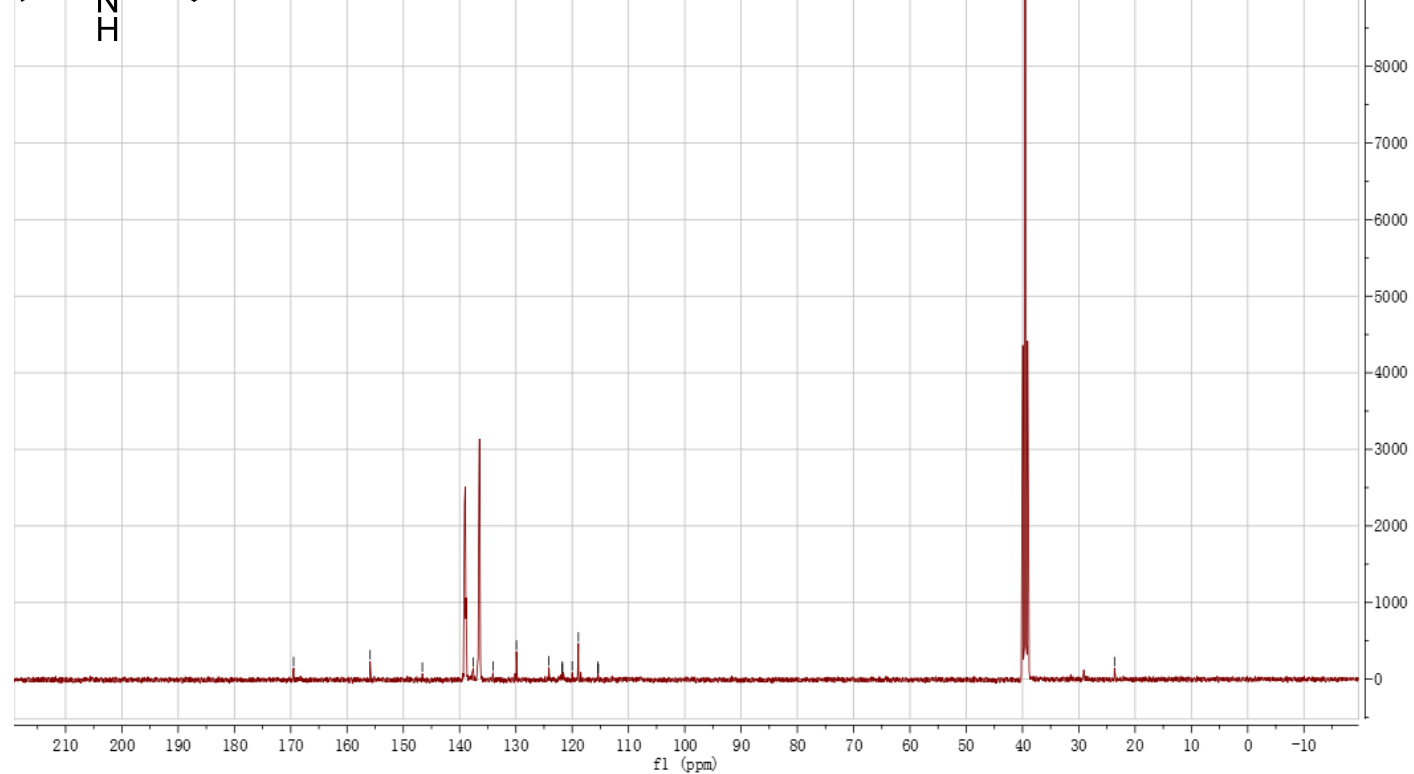


${ }^{1} \mathrm{H}$ NMR Spectra of $\mathbf{4 k}$

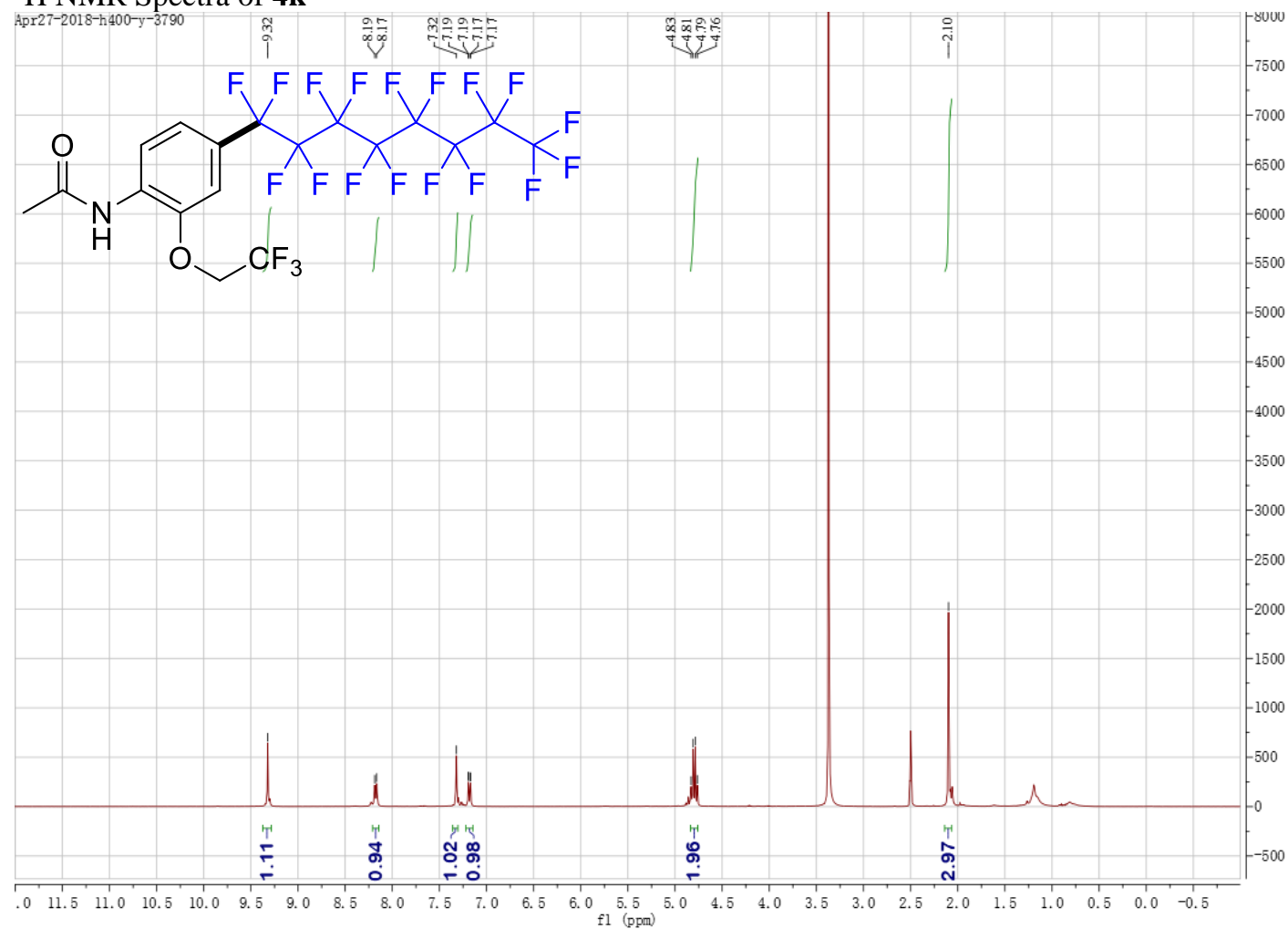

${ }^{19}$ F NMR Spectra of $\mathbf{4 k}$

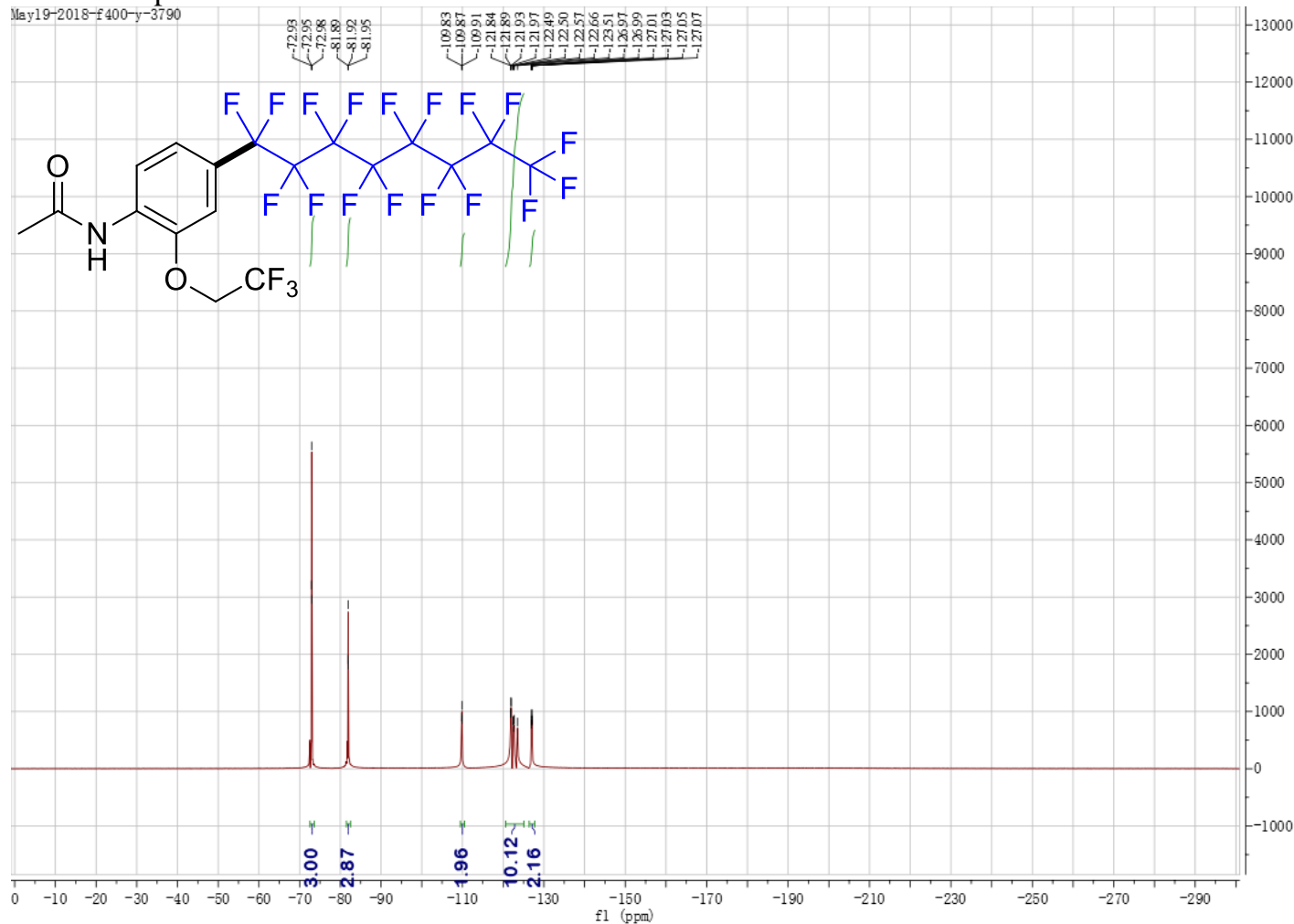


${ }^{13}$ C NMR Spectra of $\mathbf{4 k}$

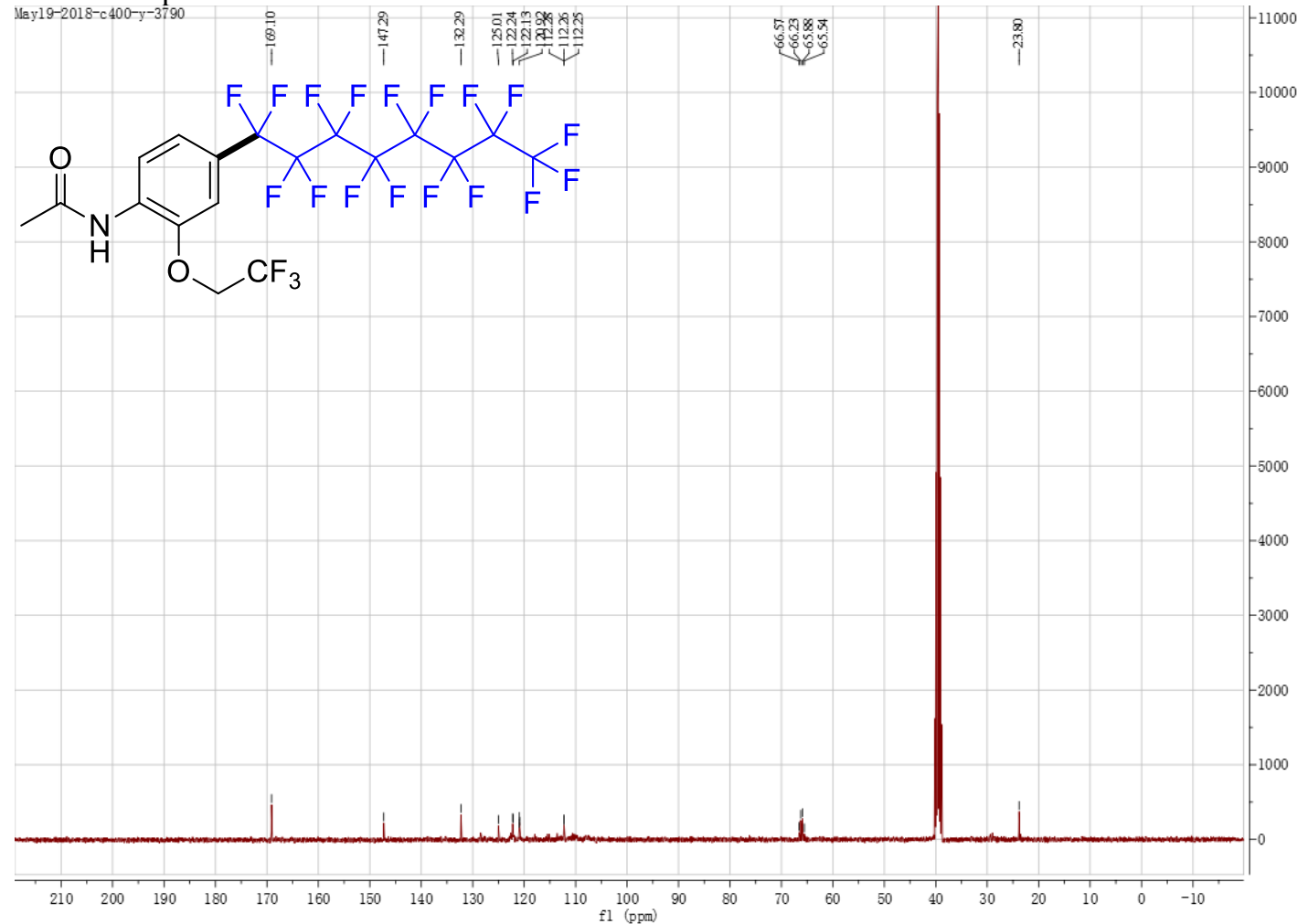

${ }^{1} \mathrm{H}$ NMR Spectra of $\mathbf{4 l}$

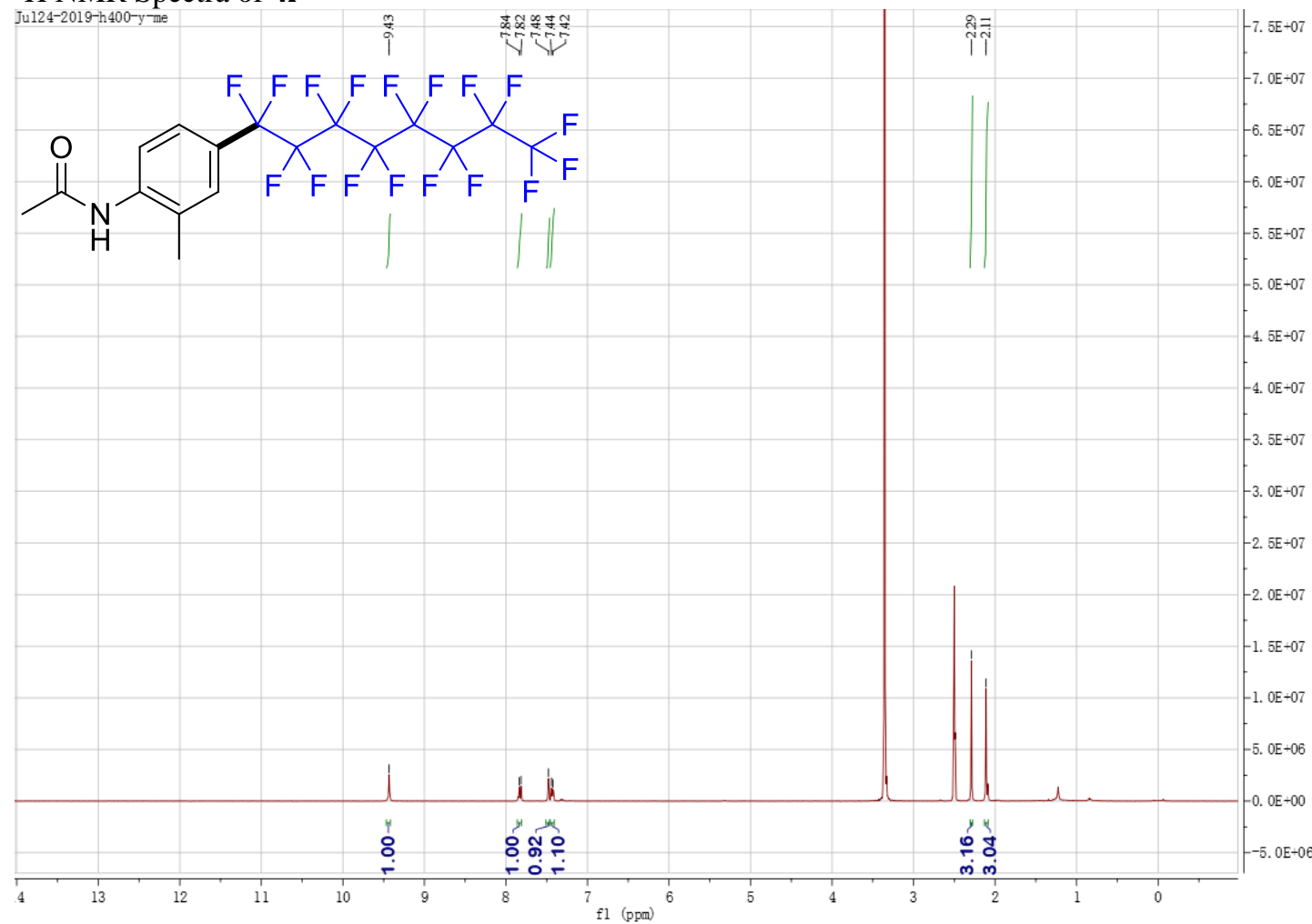


${ }^{19}$ F NMR Spectra of 41

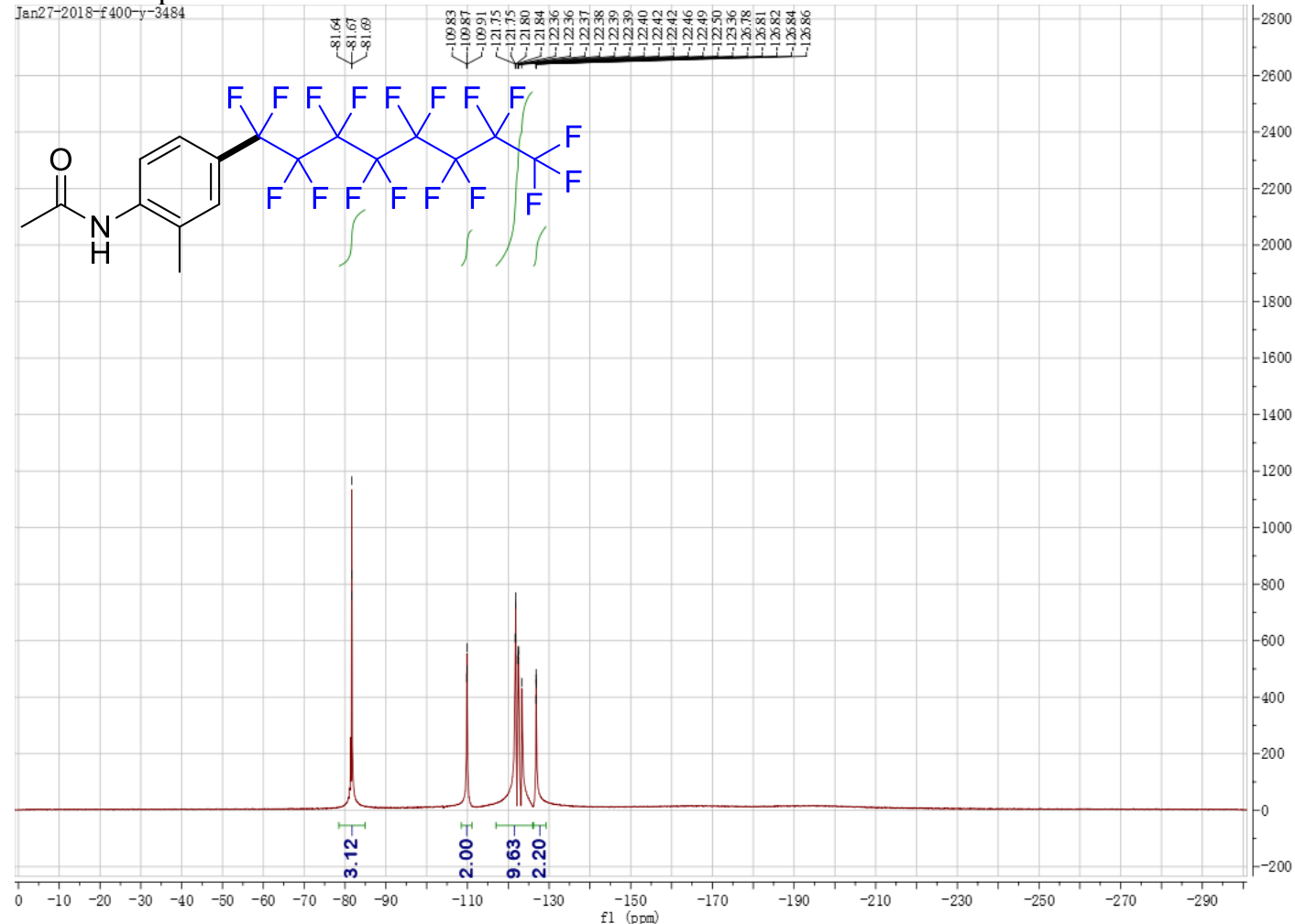

${ }^{13} \mathrm{C}$ NMR Spectra of 41

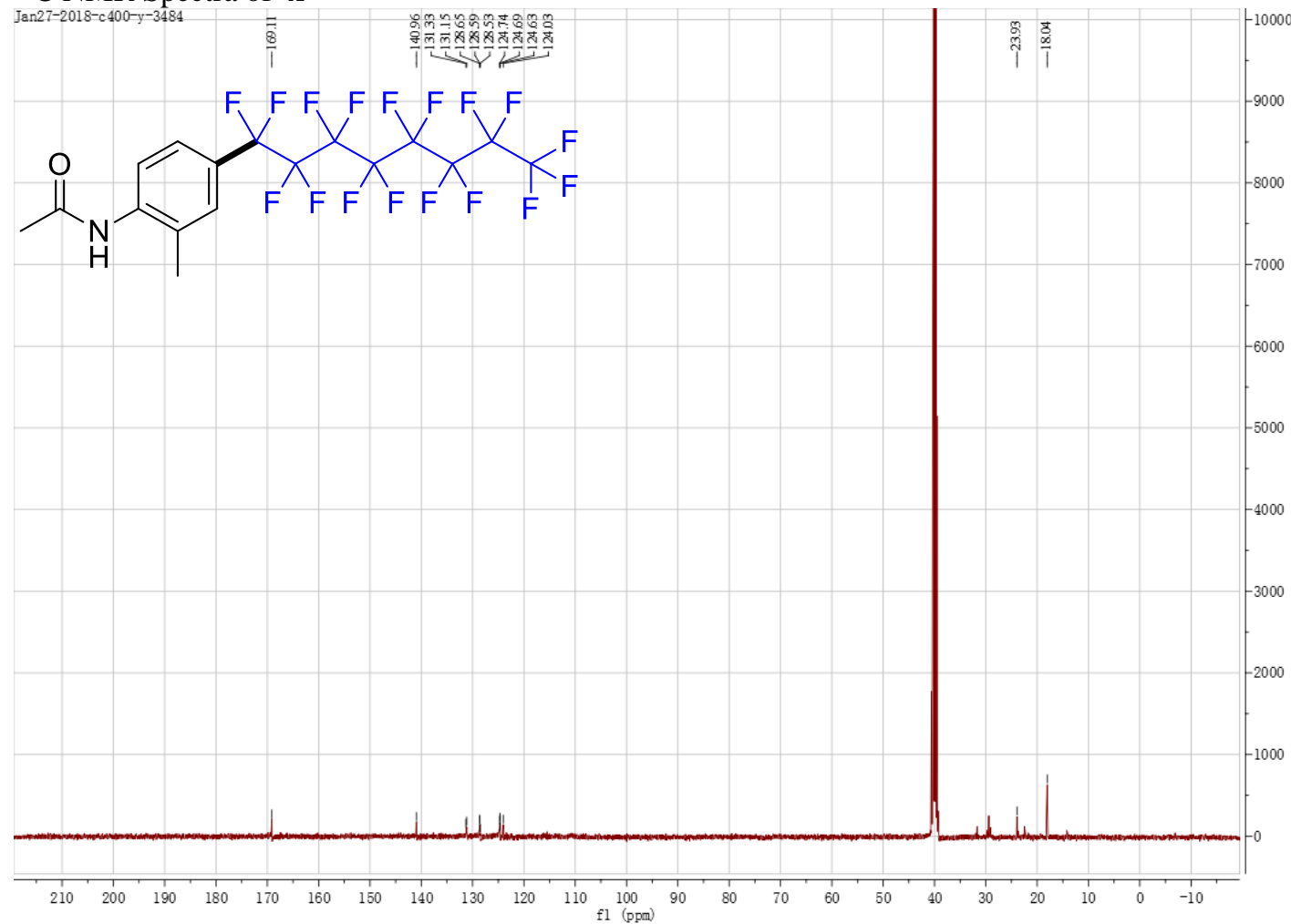


${ }^{1} \mathrm{H}$ NMR Spectra of $\mathbf{4 m}$ (Perfluorobenzene used as a co-solvent helps $4 \mathrm{~m}$ dissolve in d6-DMSO)

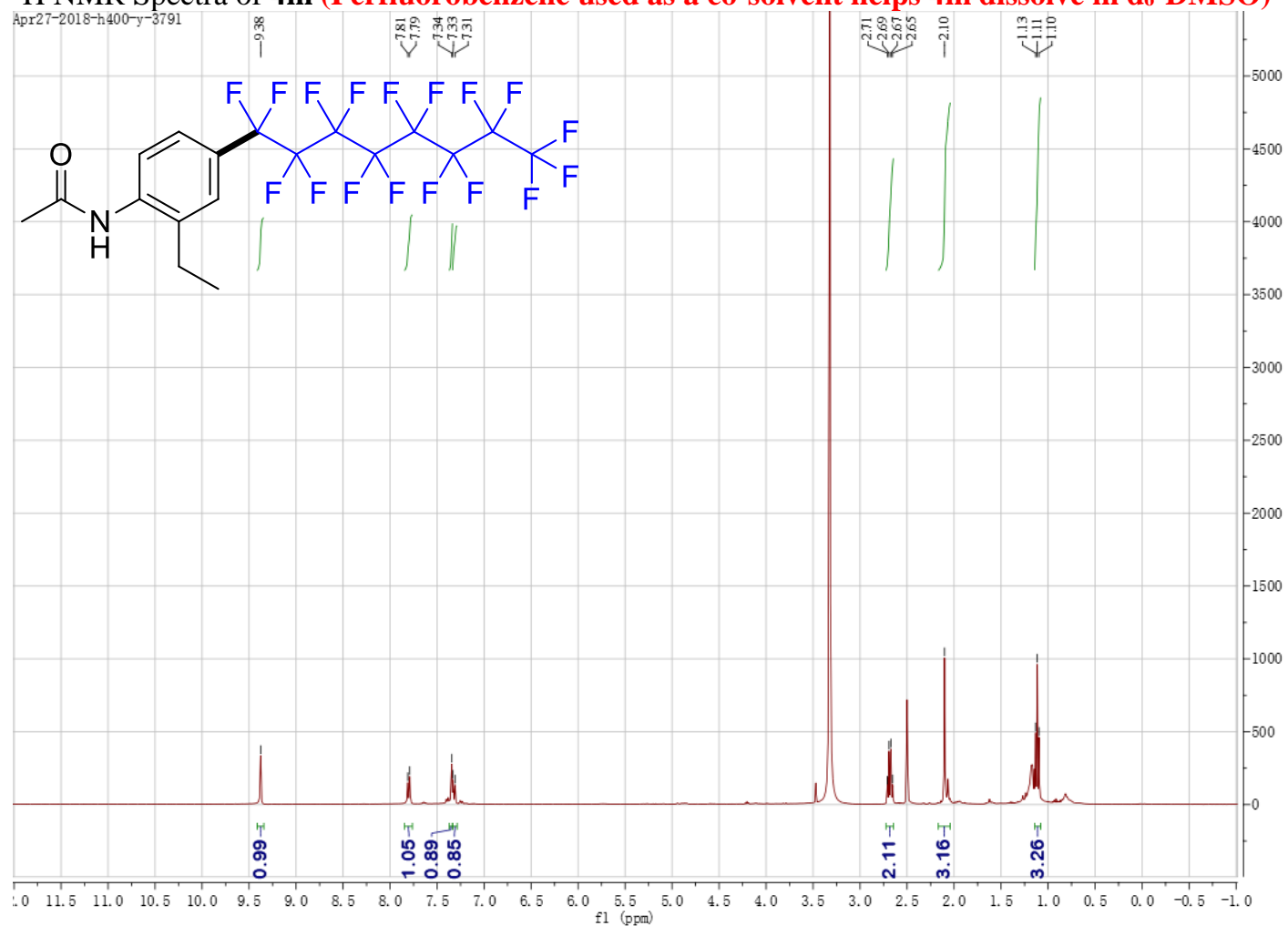

${ }^{19} \mathrm{~F}$ NMR Spectra of $\mathbf{4 m}$ (Perfluorobenzene used as a co-solvent helps $4 \mathrm{~m}$ dissolve in $\mathrm{d}_{6}$-DMSO)

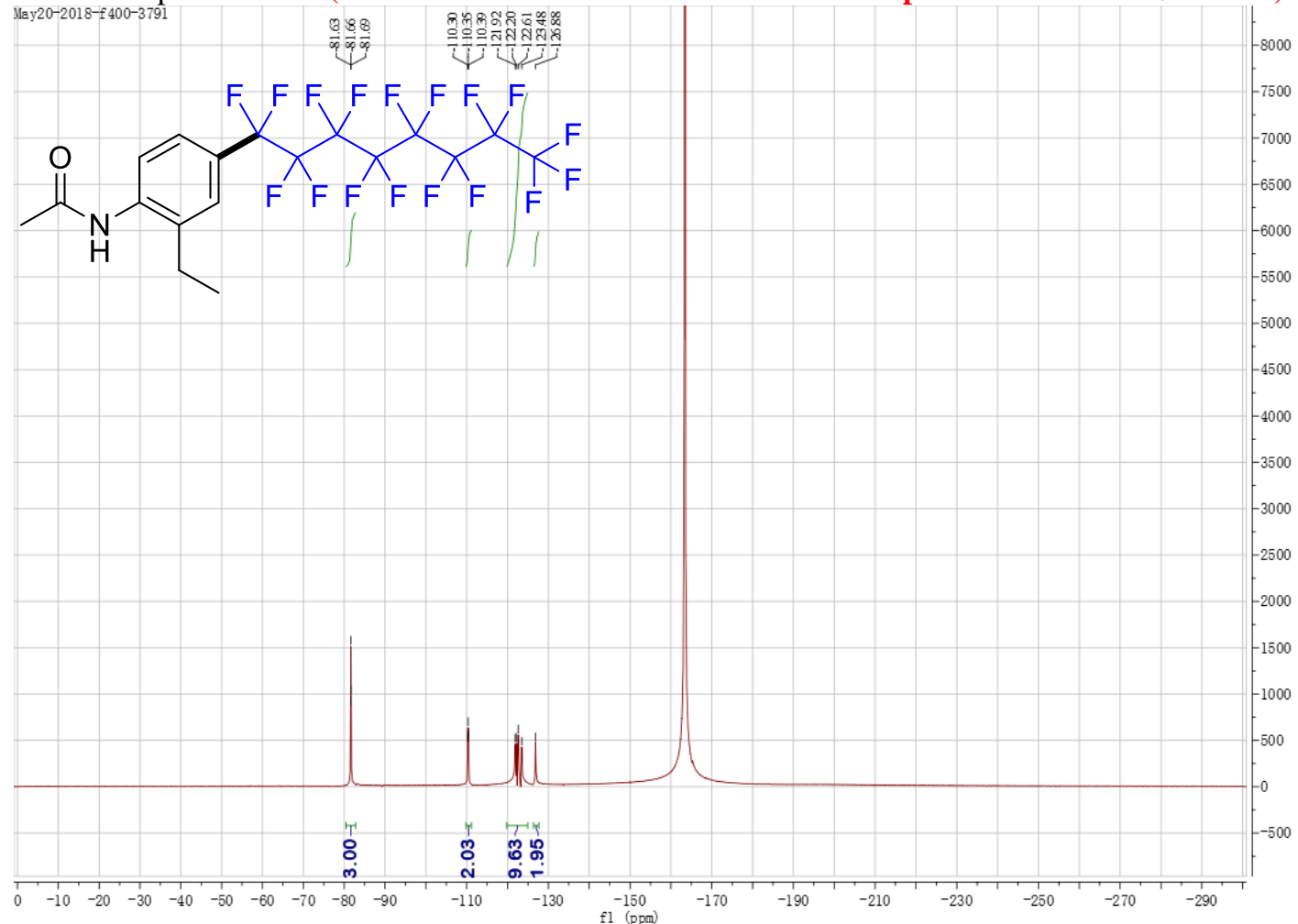


${ }^{13} \mathrm{C}$ NMR Spectra of $\mathbf{4 m}$ (Perfluorobenzene used as a co-solvent helps $4 \mathrm{~m}$ dissolve in $\mathrm{d}_{6}$-DMSO)

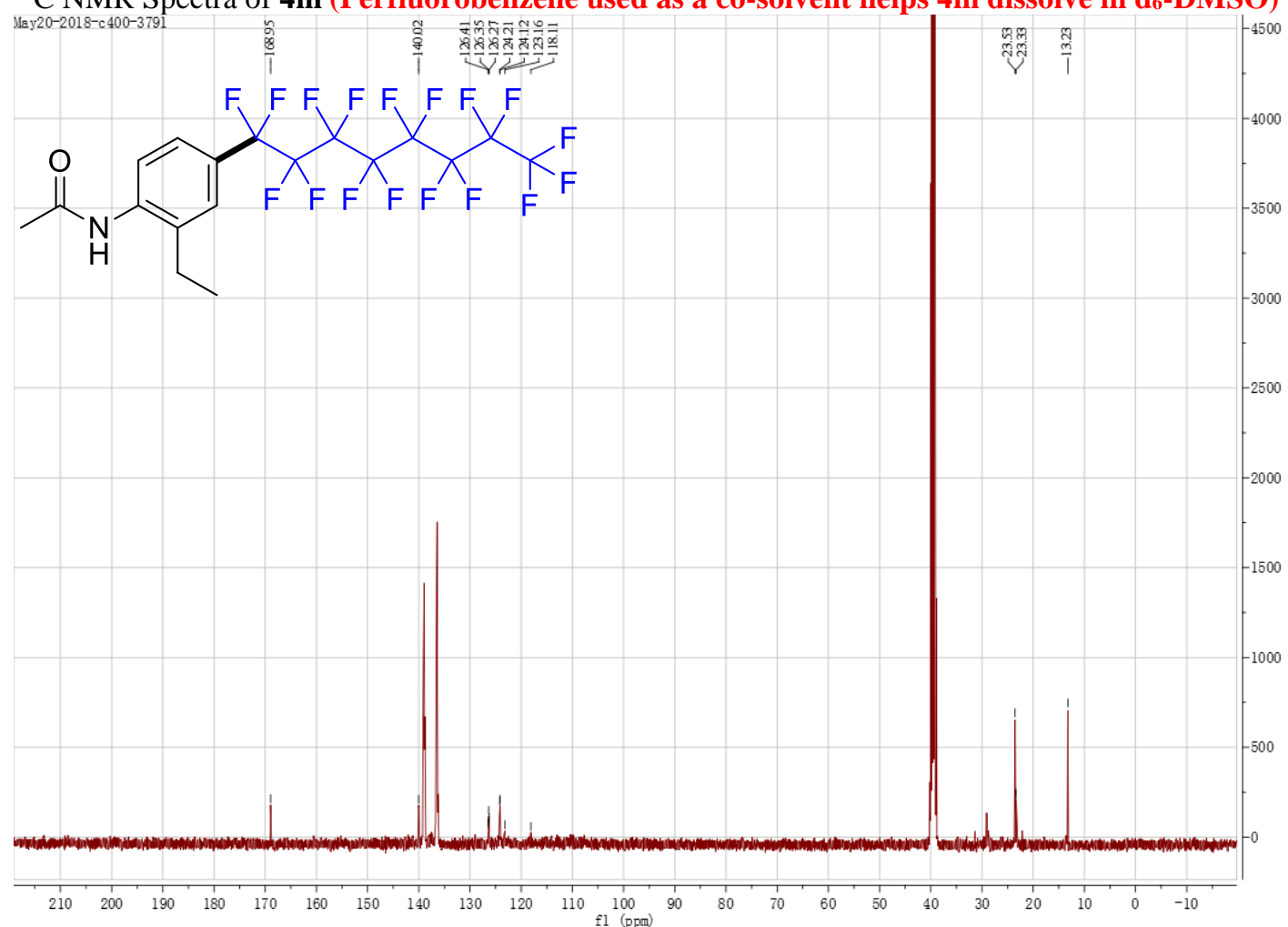

${ }^{1} \mathrm{H}$ NMR Spectra of $4 \mathbf{n}$ (Perfluorobenzene used as a co-solvent helps $4 \mathbf{n}$ dissolve in d6-DMSO)

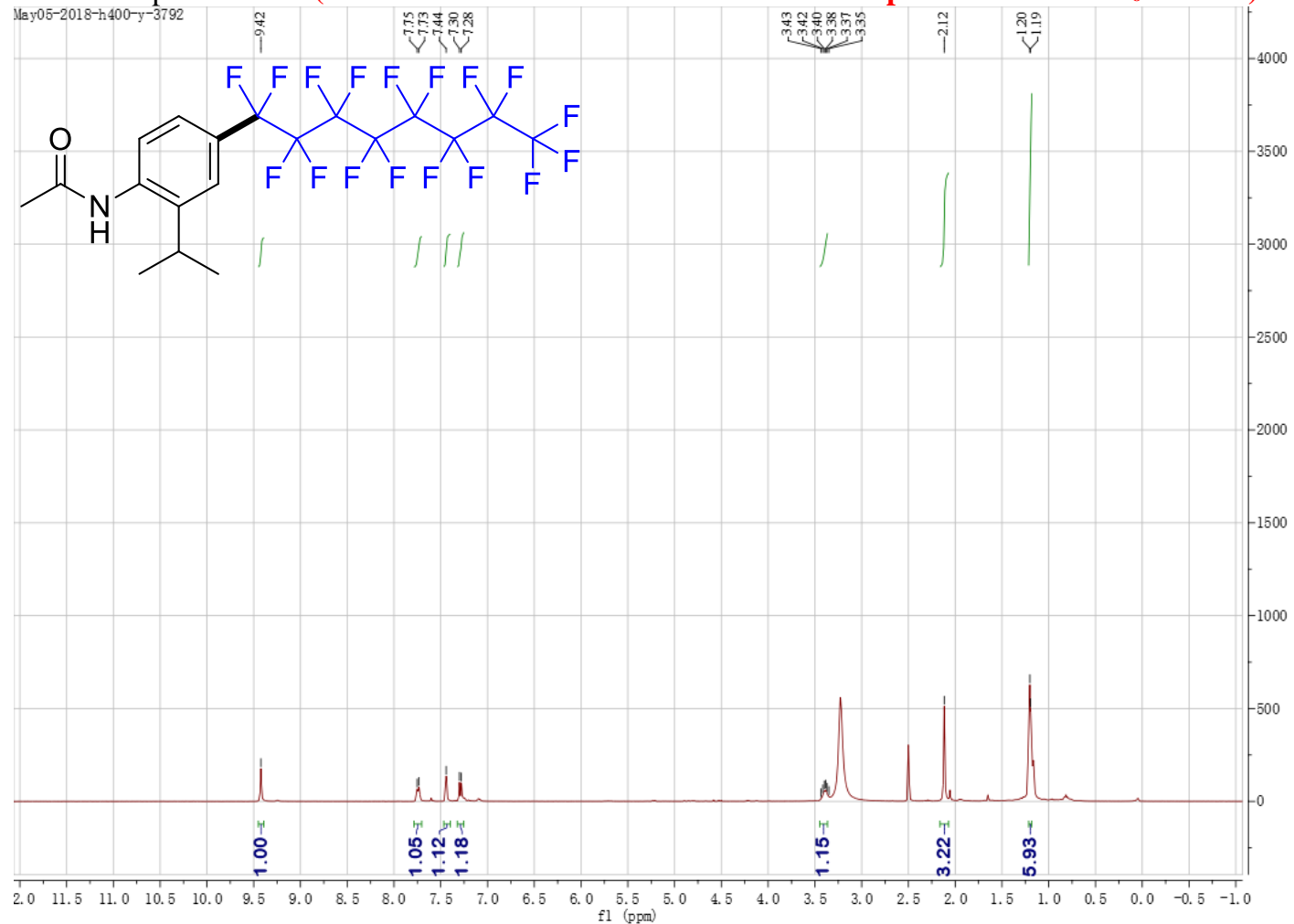


${ }^{19} \mathrm{~F}$ NMR Spectra of 4n (Perfluorobenzene used as a co-solvent helps 4n dissolve in $\mathrm{d}_{6}$-DMSO)

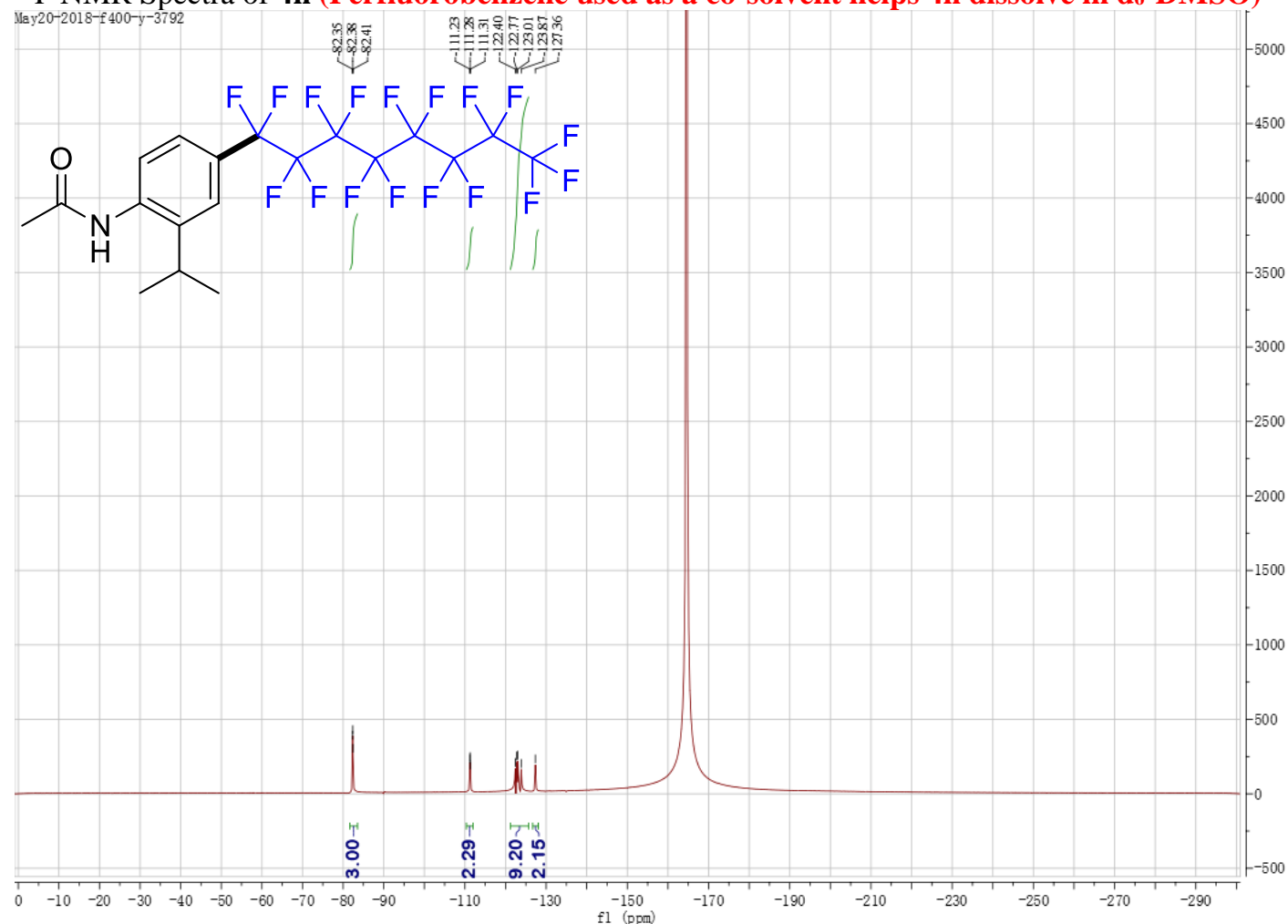

${ }^{13} \mathrm{C}$ NMR Spectra of $4 \mathrm{n}$ (Perfluorobenzene used as a co-solvent helps $4 \mathbf{n}$ dissolve in $\mathbf{d}_{6}$-DMSO)

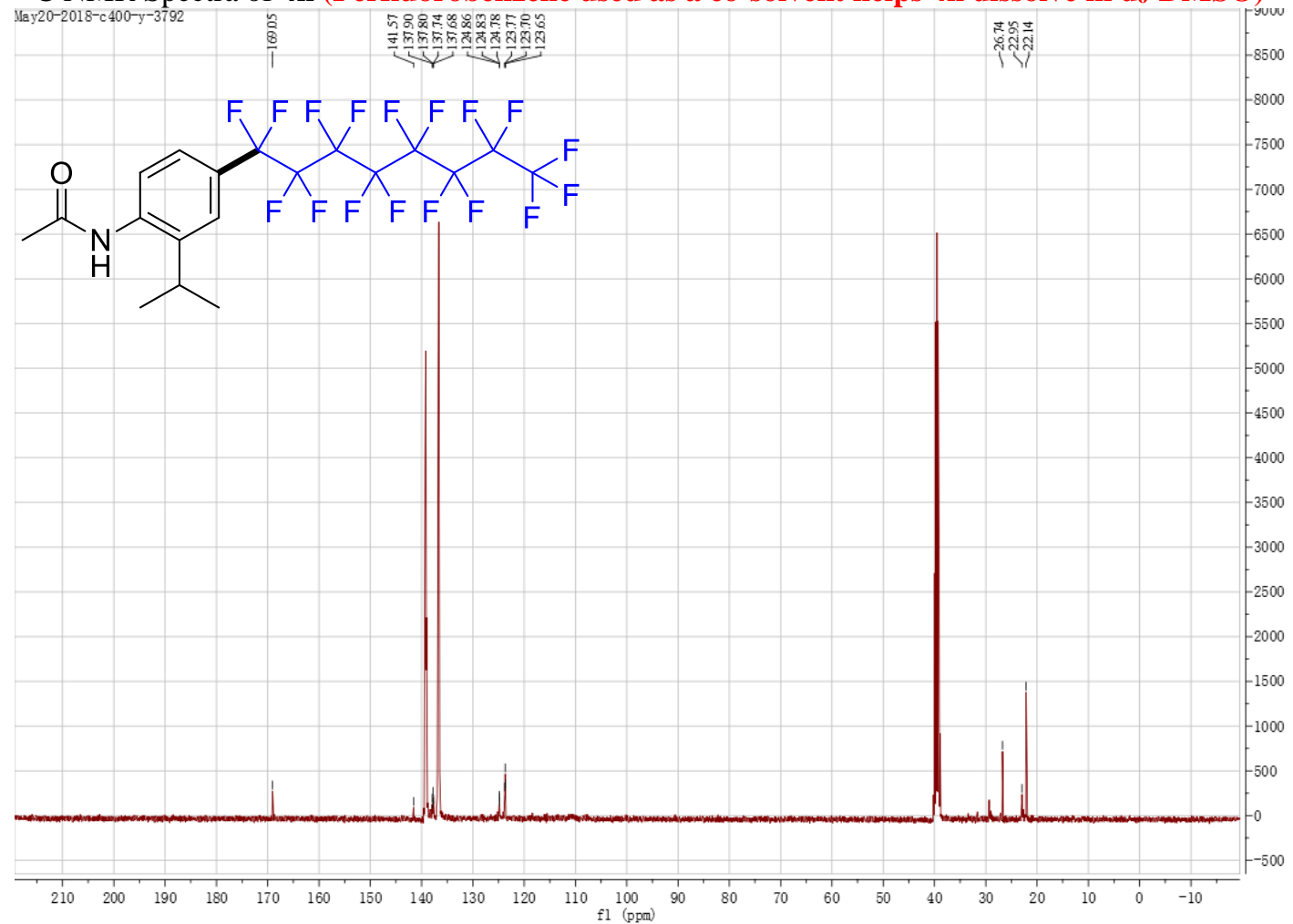


${ }^{1} \mathrm{H}$ NMR, ${ }^{19} \mathrm{~F}$ NMR \& ${ }^{13} \mathrm{C}$ NMR Spectra of 40 (Perfluorobenzene used as a co-solvent helps $4 \mathrm{o}$ dissolve in $\mathrm{d}_{6}$-DMSO)

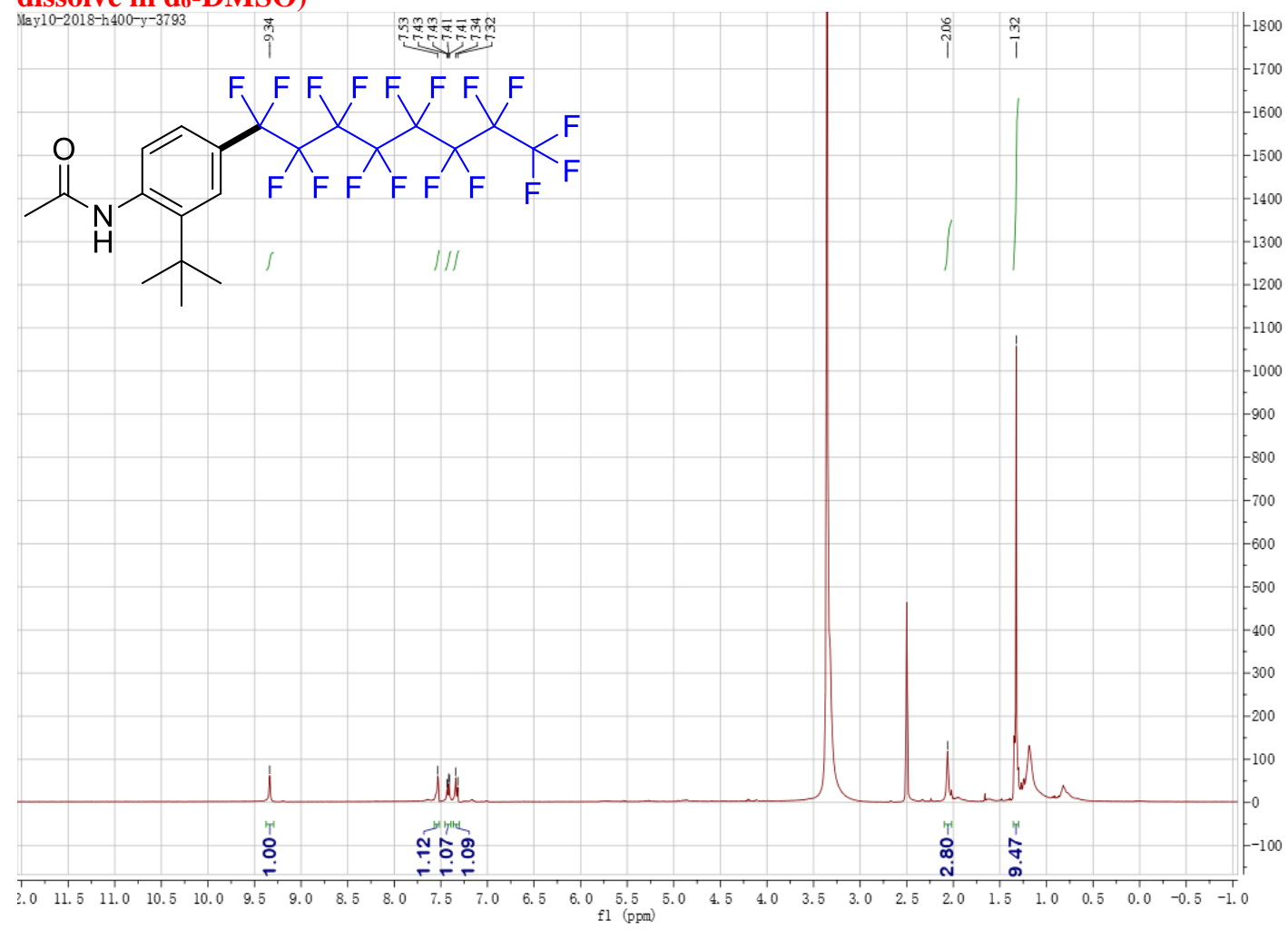

${ }^{19} \mathrm{~F}$ NMR Spectra of 40 (Perfluorobenzene used as a co-solvent helps 40 dissolve in d6-DMSO)

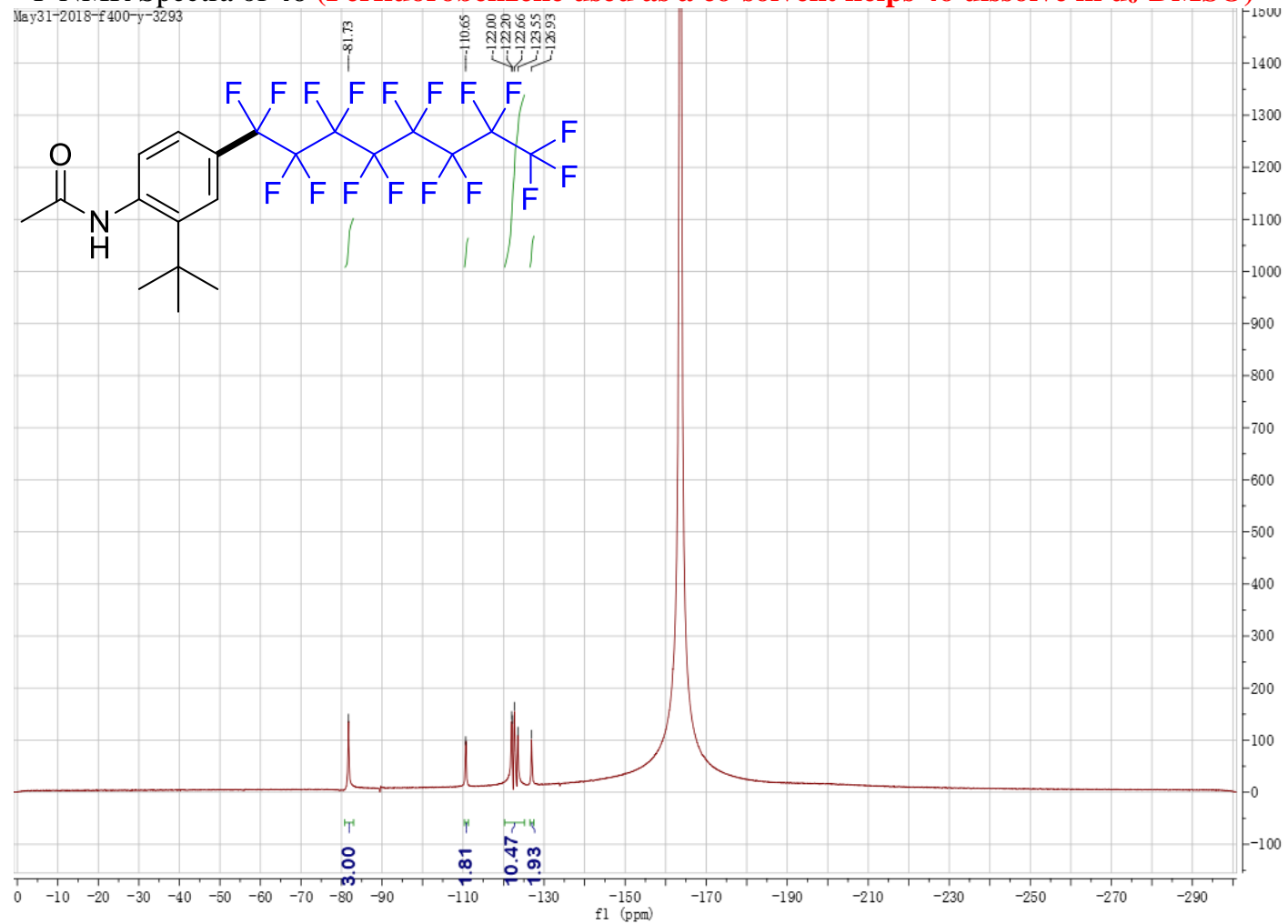


${ }^{13} \mathrm{C}$ NMR Spectra of 40 (Perfluorobenzene used as a co-solvent helps 40 dissolve in $\mathrm{d}_{6}$-DMSO)

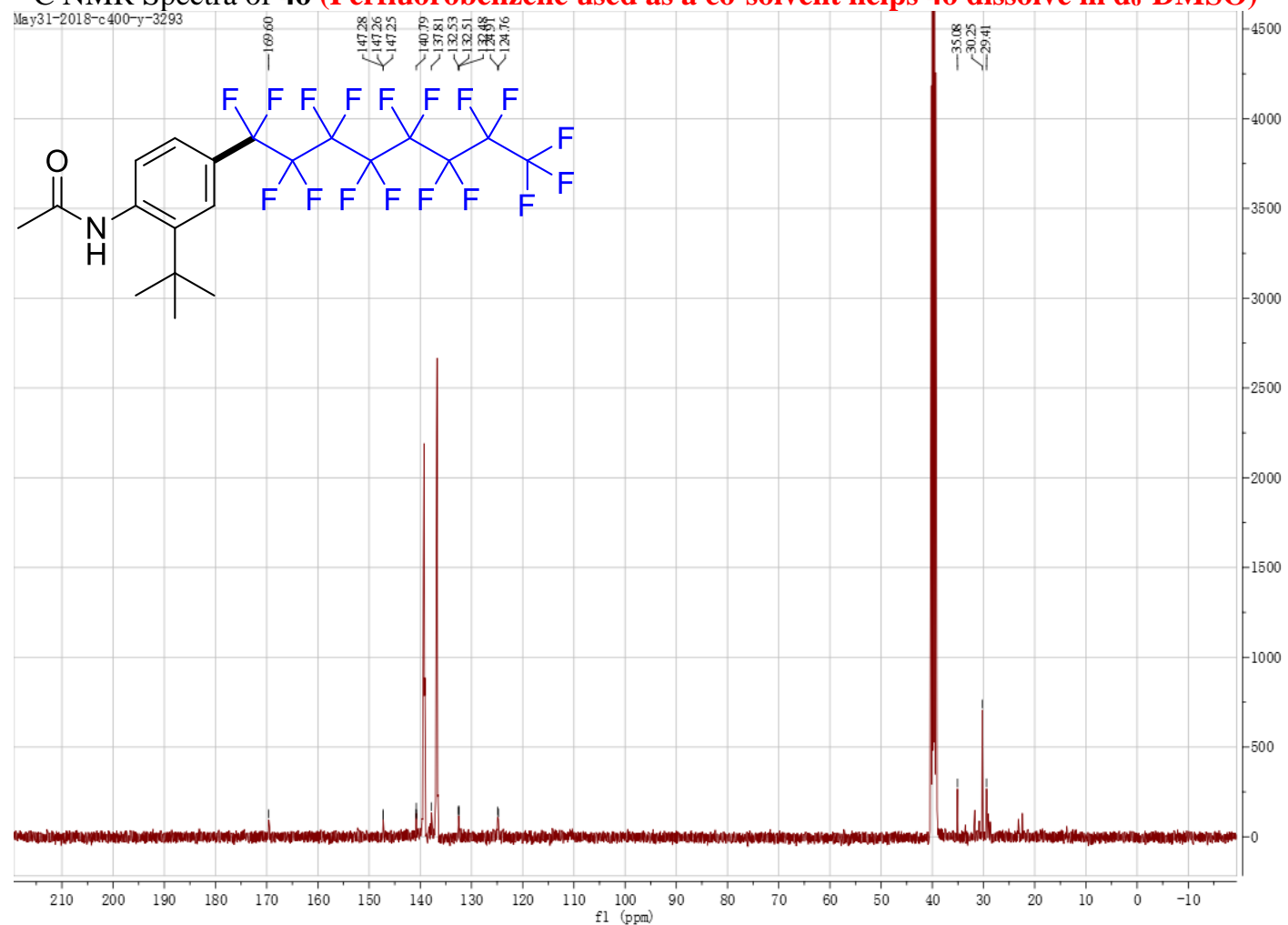

${ }^{1} \mathrm{H}$ NMR Spectra of $\mathbf{4 p}$ (Perfluorobenzene used as a co-solvent helps $4 p$ dissolve in $\mathrm{d}_{6}$-DMSO)

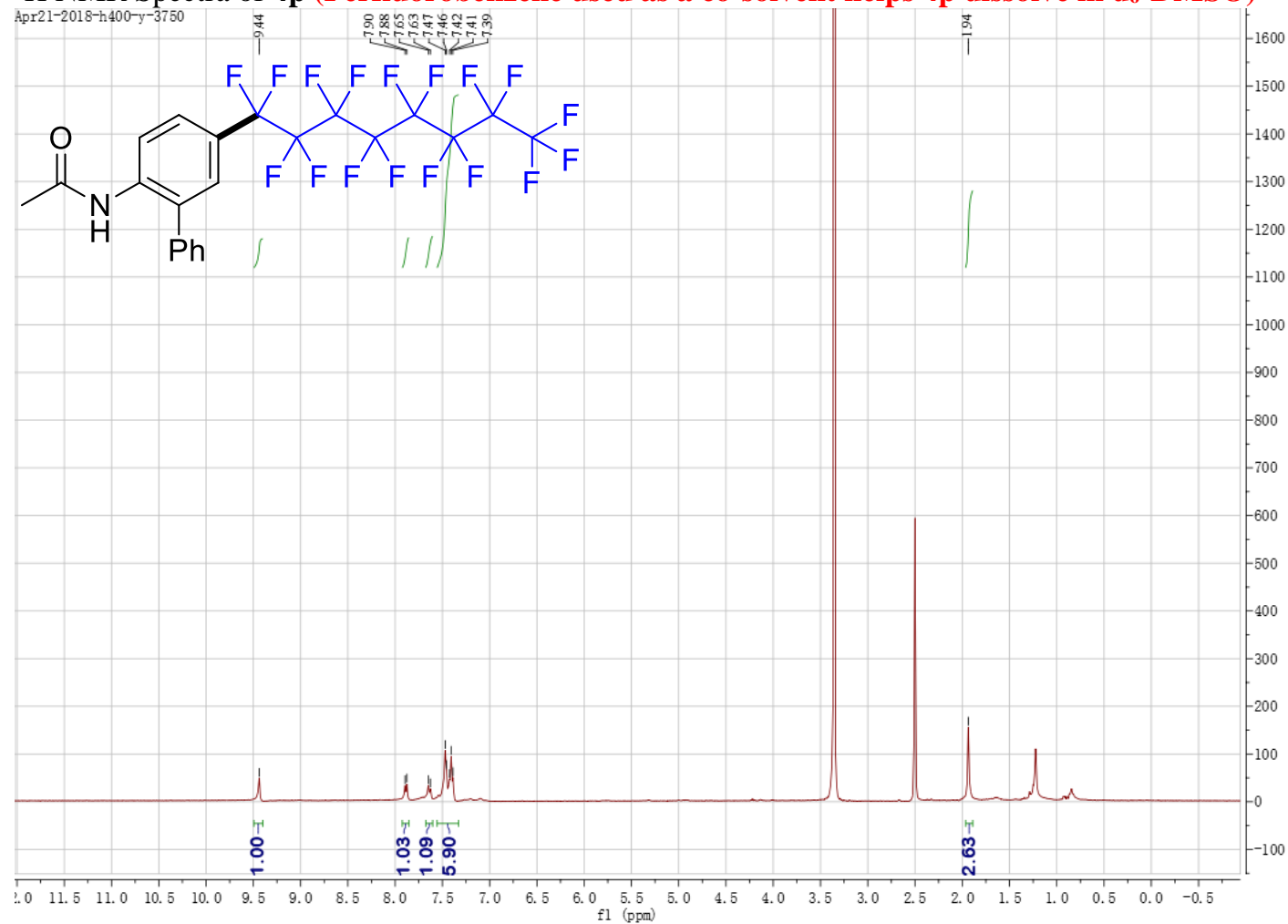


${ }^{19} \mathrm{~F}$ NMR Spectra of 4p (Perfluorobenzene used as a co-solvent helps 4p dissolve in $\mathrm{d}_{6}$-DMSO)

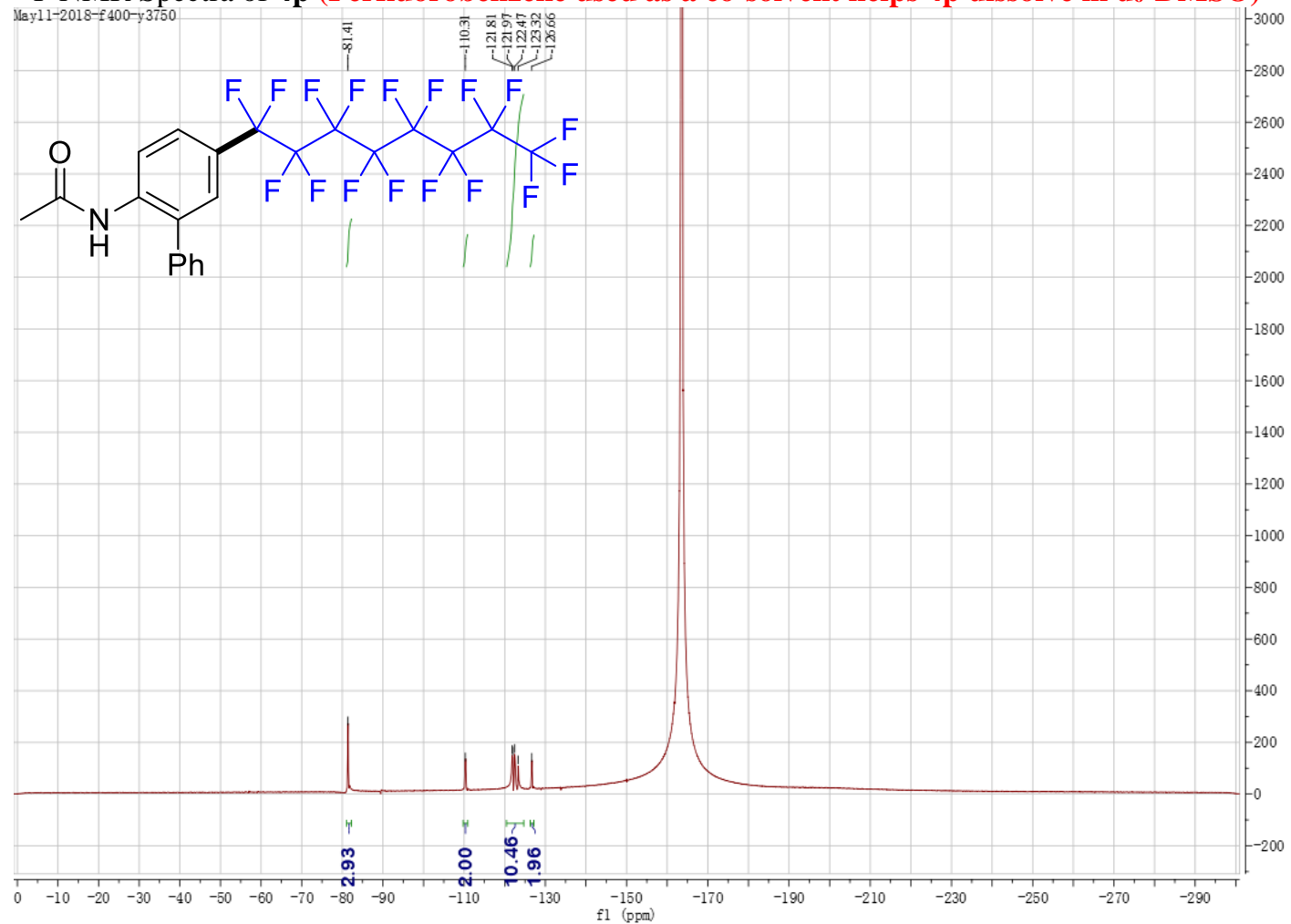

${ }^{13} \mathrm{C}$ NMR Spectra of $4 p$ (Perfluorobenzene used as a co-solvent helps $4 p$ dissolve in $\mathbf{d}_{6}$-DMSO)

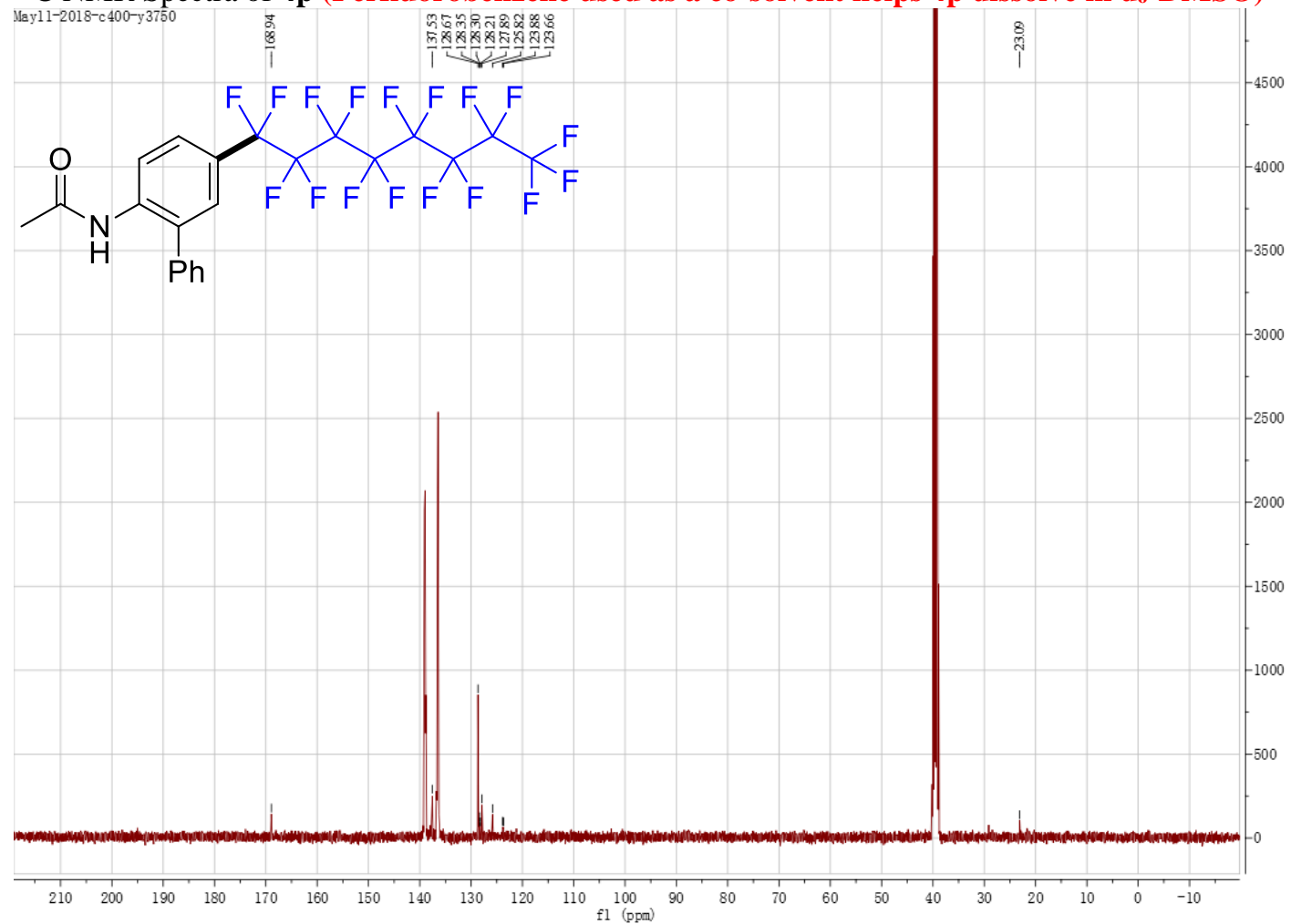


${ }^{1} \mathrm{H}$ NMR Spectra of $\mathbf{4 q}$

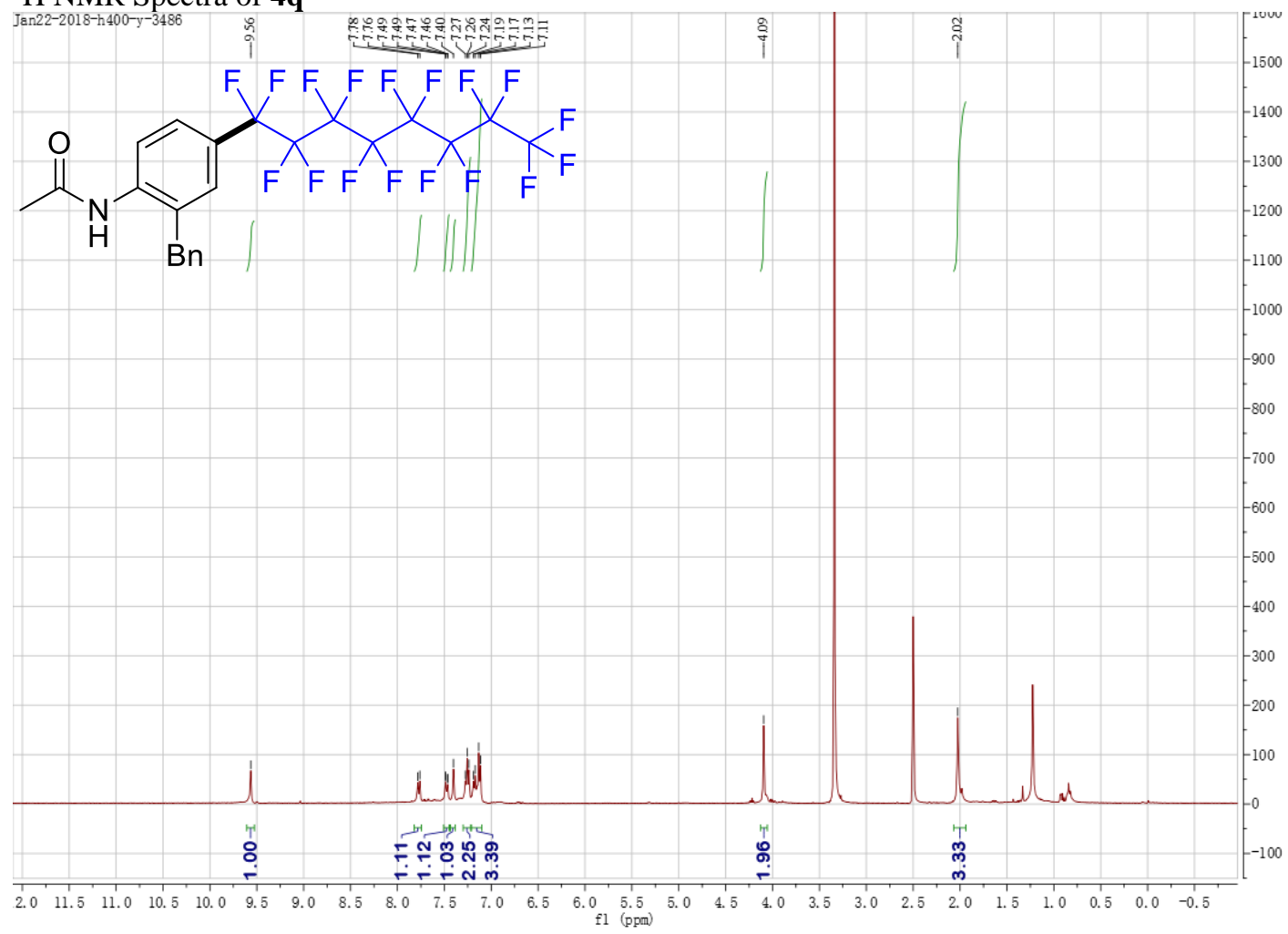

${ }^{19}$ F NMR Spectra of $\mathbf{4 q}$ Jan27-201 8-f 400-y-3486<smiles>CC(=O)Nc1ccc(C(F)(F)C(F)(F)C(F)(F)C(F)(F)C(F)(F)C(F)(F)C(F)(F)C(F)(F)F)cc1Br</smiles> 
${ }^{13} \mathrm{C}$ NMR Spectra of $\mathbf{4 q}$

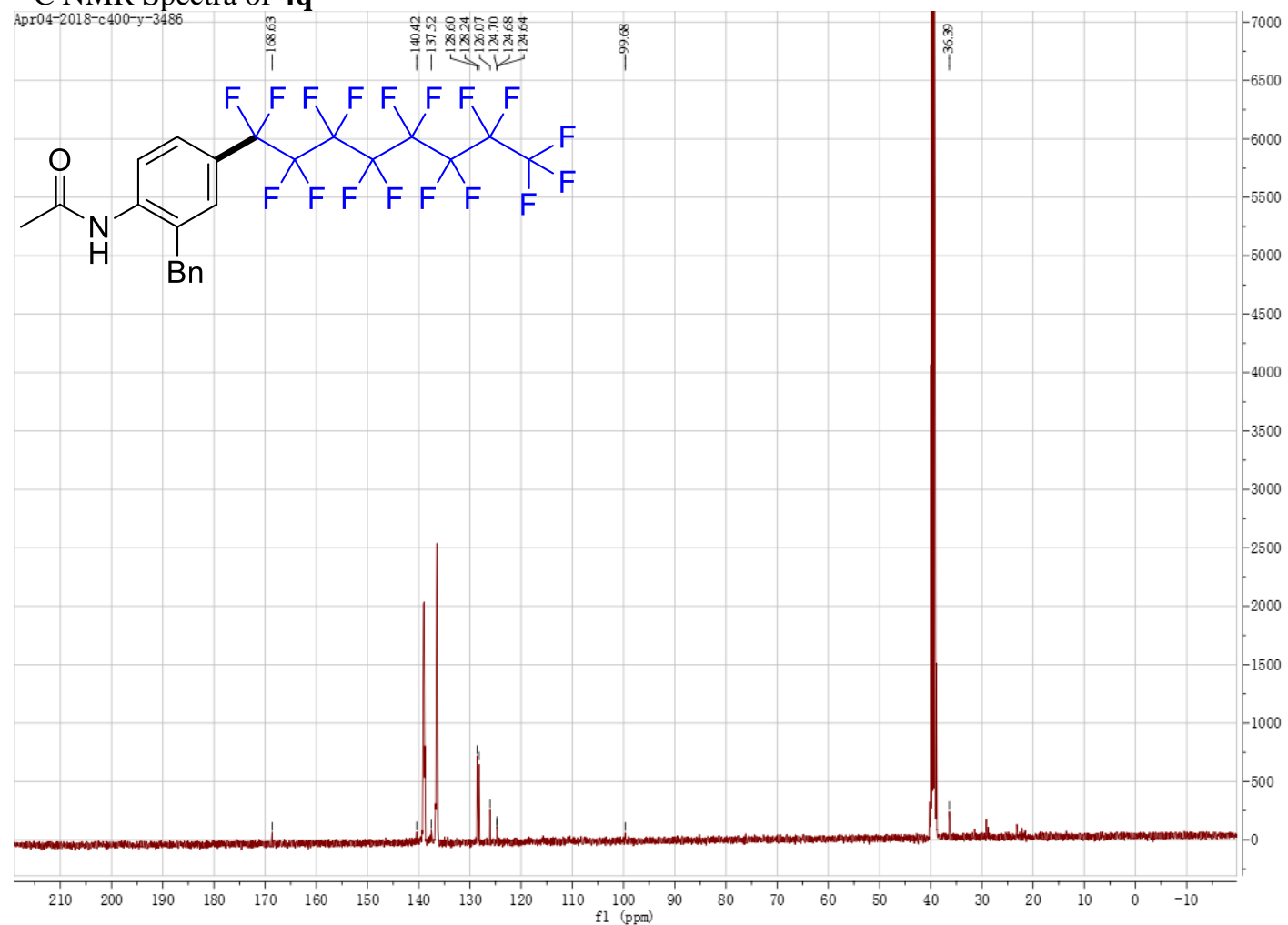

${ }^{1} \mathrm{H}$ NMR Spectra of $\mathbf{4 r}$

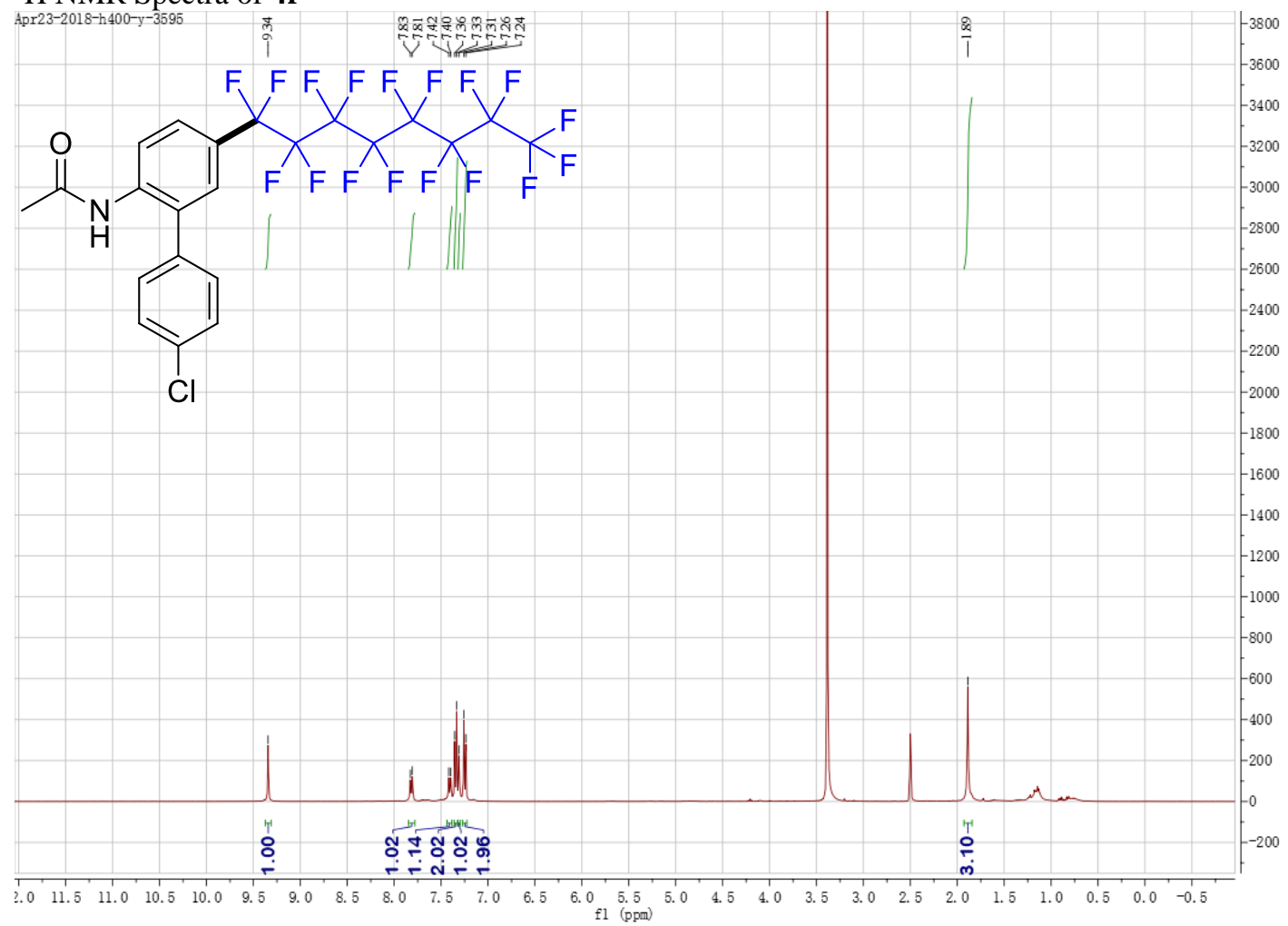


${ }^{19} \mathrm{~F}$ NMR Spectra of $\mathbf{4 r}$

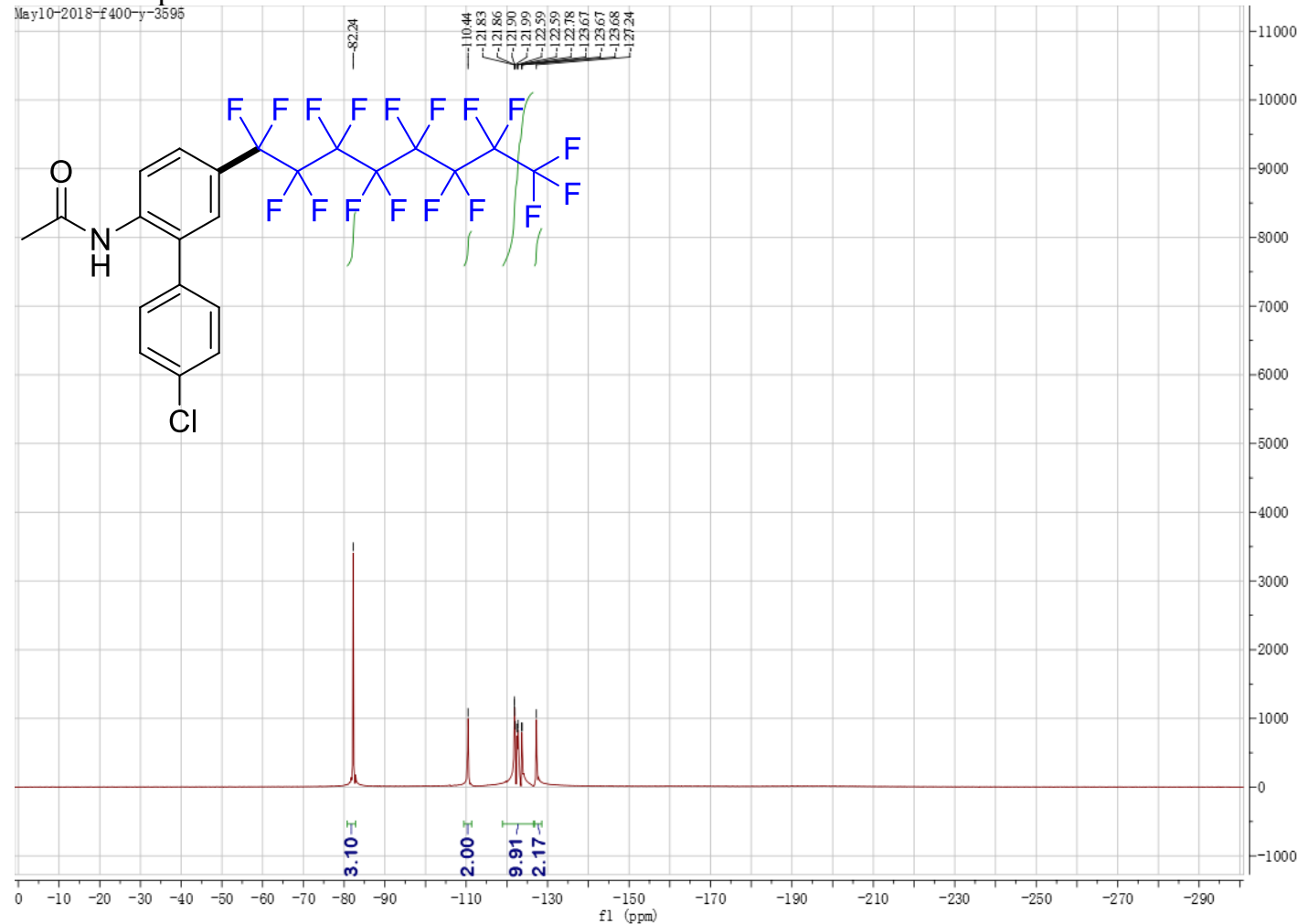

${ }^{13} \mathrm{C}$ NMR Spectra of $\mathbf{4 r}$

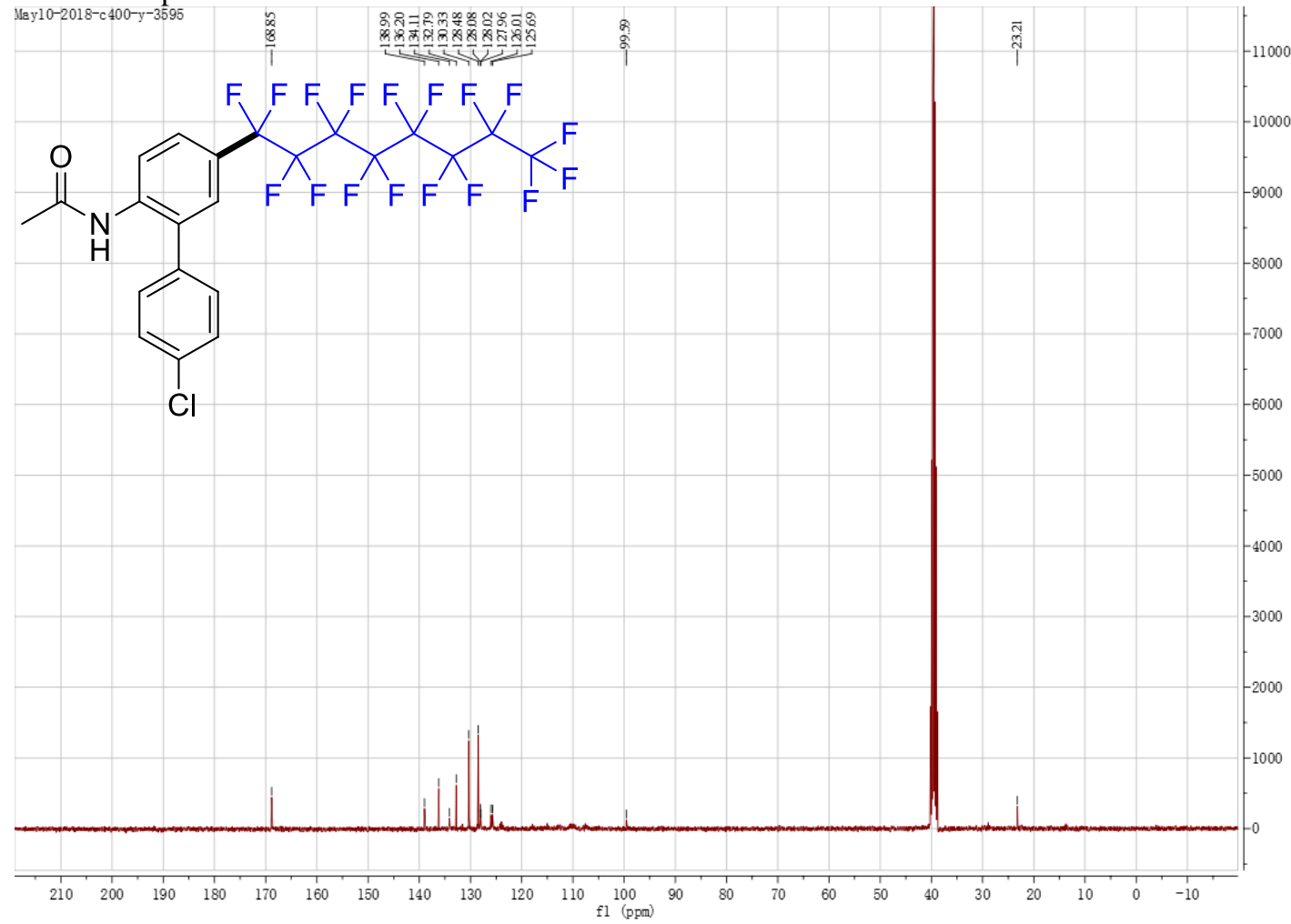


${ }^{1} \mathrm{H}$ NMR Spectra of $4 \mathbf{s}$ (Perfluorobenzene used as a co-solvent helps $4 \mathbf{s}$ dissolve in $\mathrm{d}_{6}$-DMSO)

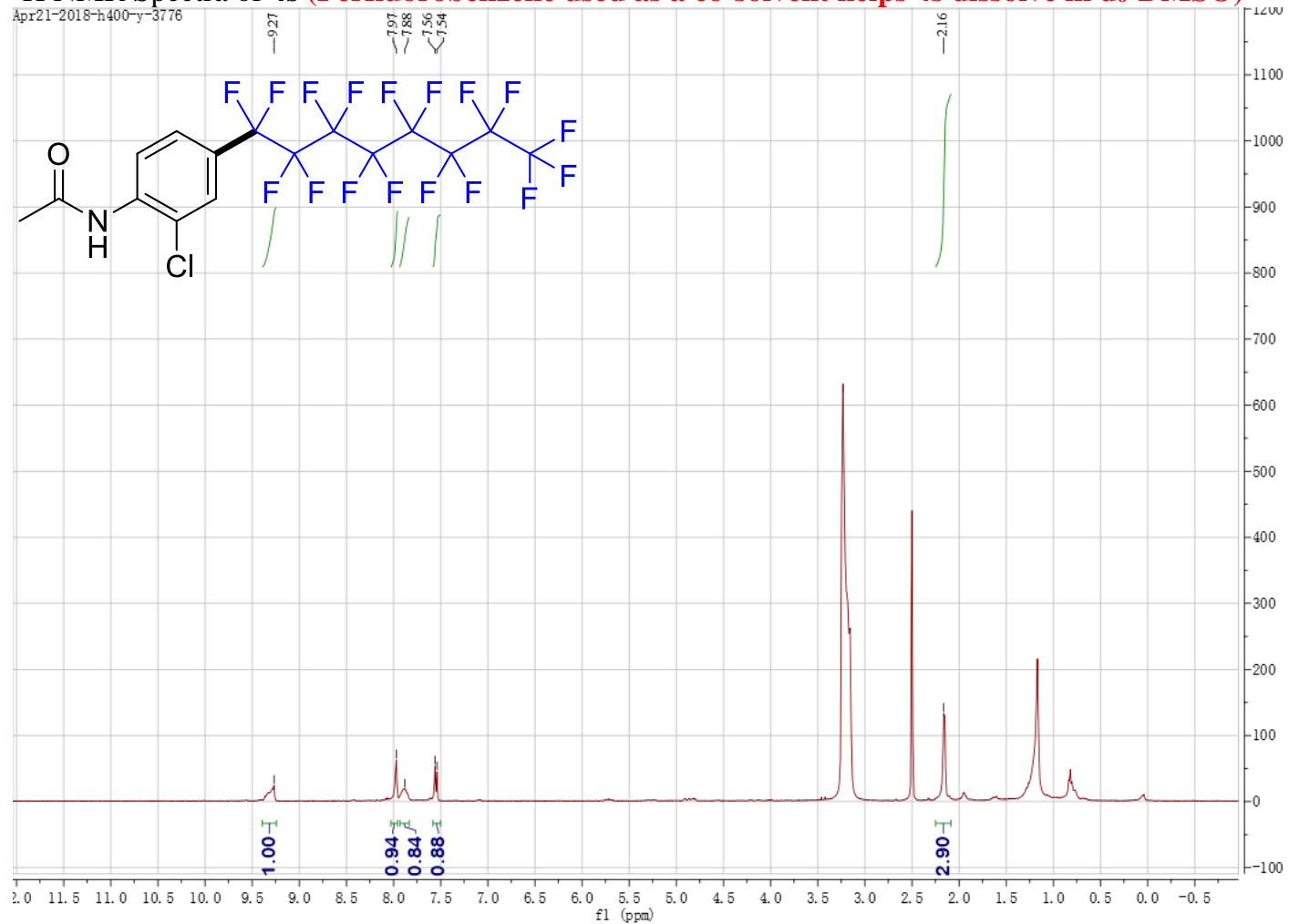

${ }^{19} \mathrm{~F}$ NMR Spectra of $\mathbf{4 s}$ (Perfluorobenzene used as a co-solvent helps $4 \mathrm{~s}$ dissolve in $\mathrm{d}_{6}$-DMSO) Apr23-2018- $400-y-3776$

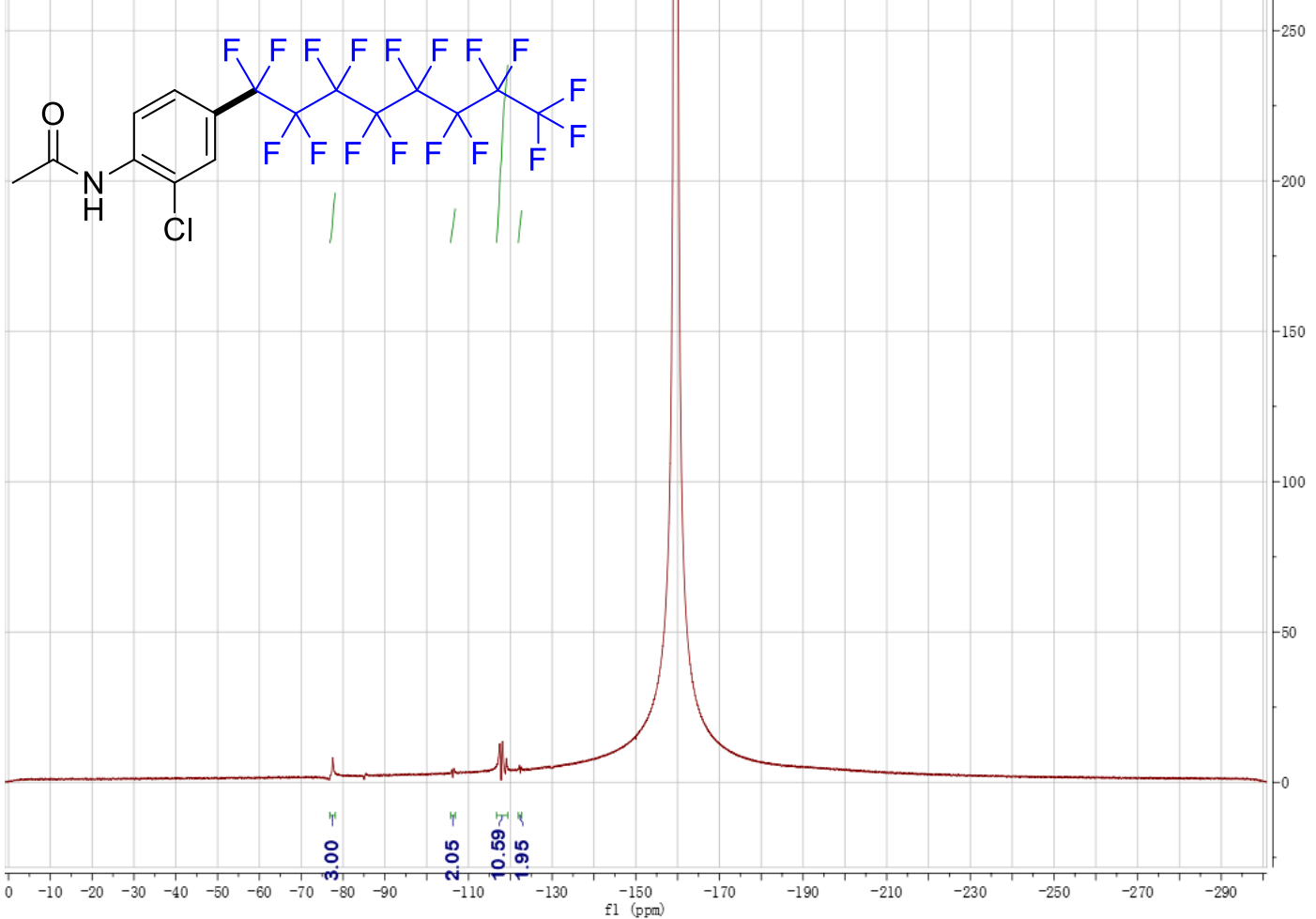


${ }^{13} \mathrm{C}$ NMR Spectra of $4 \mathrm{~s}$ (Perfluorobenzene used as a co-solvent helps $4 \mathrm{~s}$ dissolve in $\mathrm{d}_{6}$-DMSO)

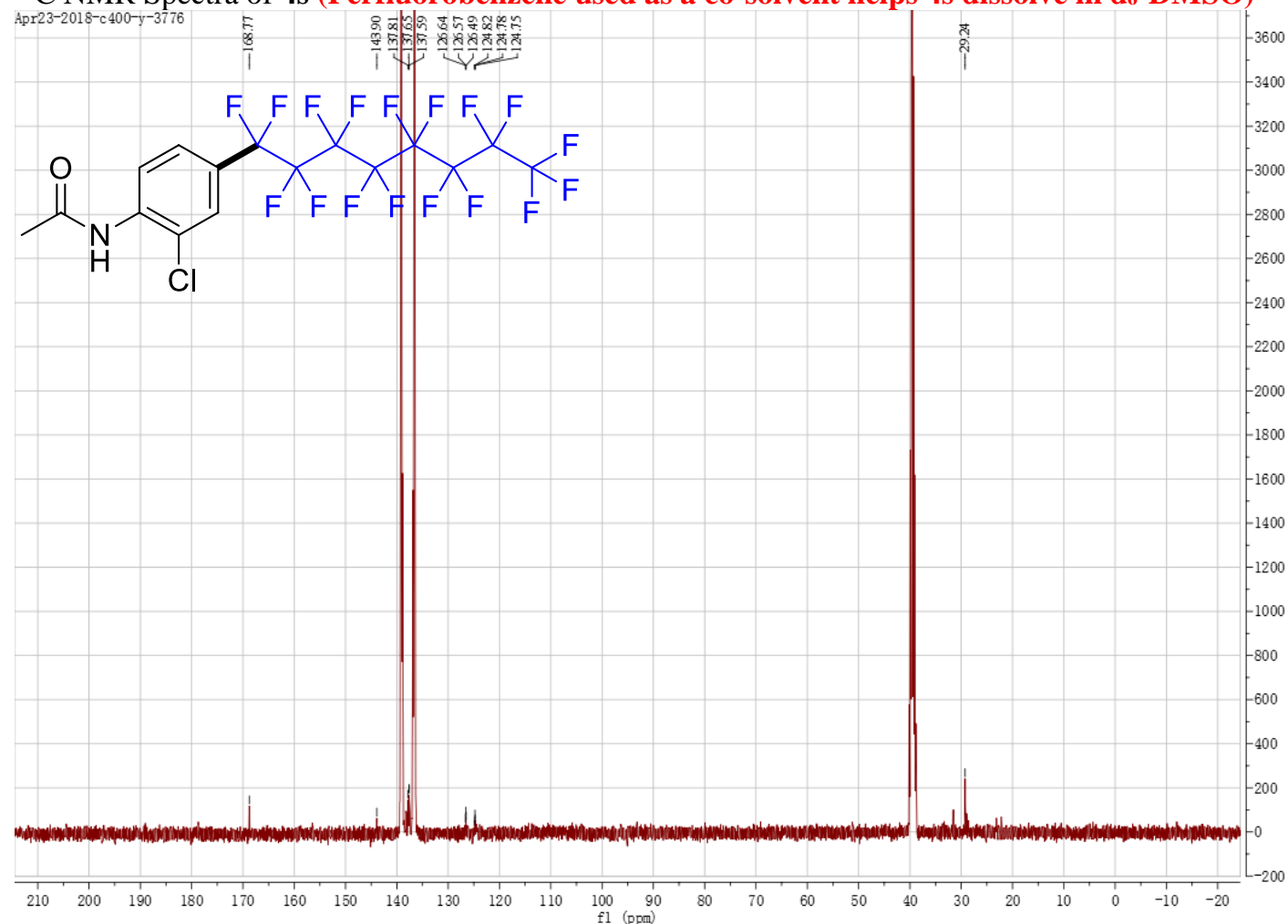

${ }^{1} \mathrm{H}$ NMR Spectra of $4 \mathrm{t}$ (Perfluorobenzene used as a co-solvent helps $4 \mathrm{t}$ dissolve in $\mathrm{d}_{6}$-DMSO)

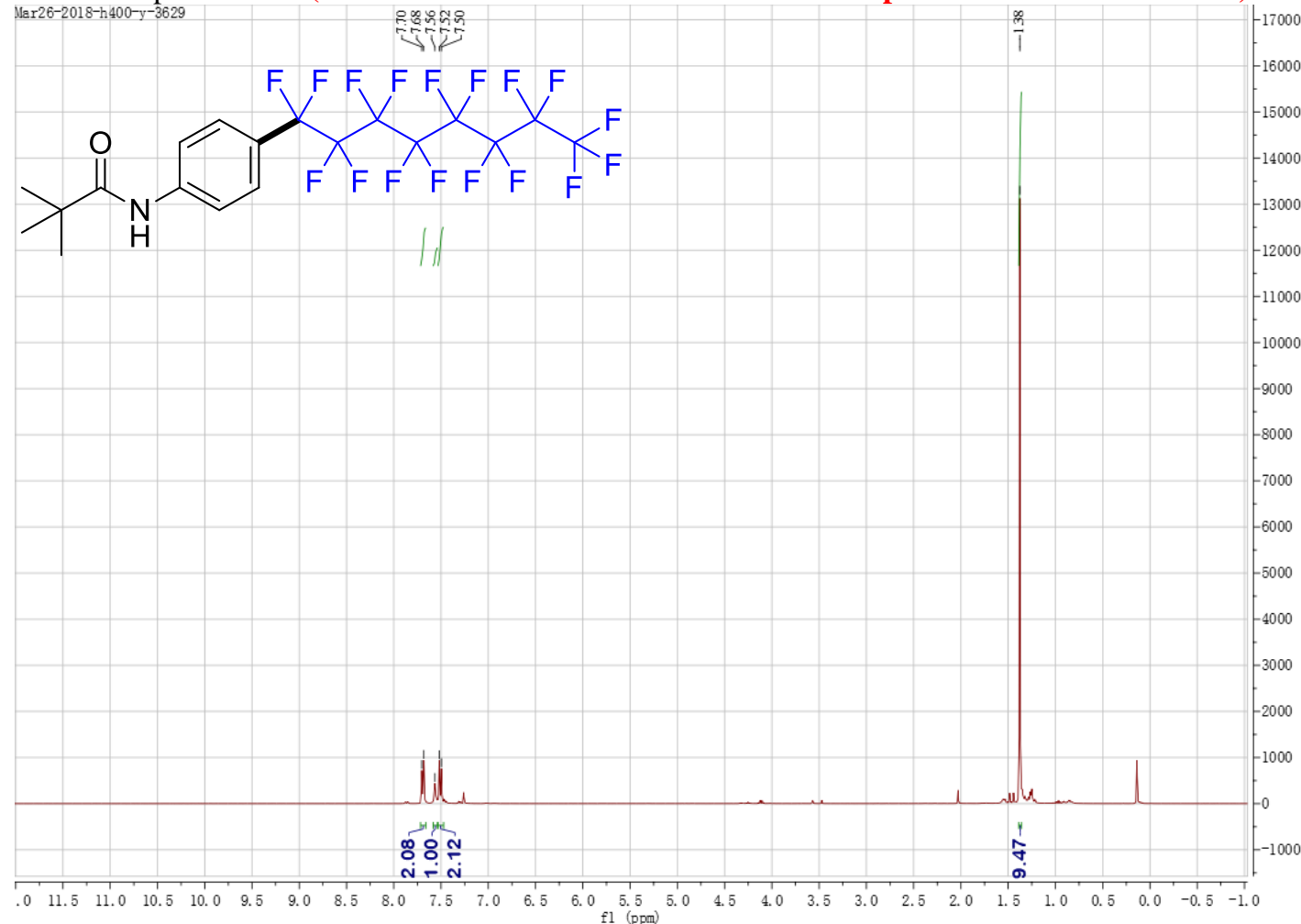


${ }^{19} \mathrm{~F}$ NMR Spectra of $\mathbf{4 t}$ (Perfluorobenzene used as a co-solvent helps $4 \mathrm{t}$ dissolve in $\mathrm{d}_{6}$-DMSO)

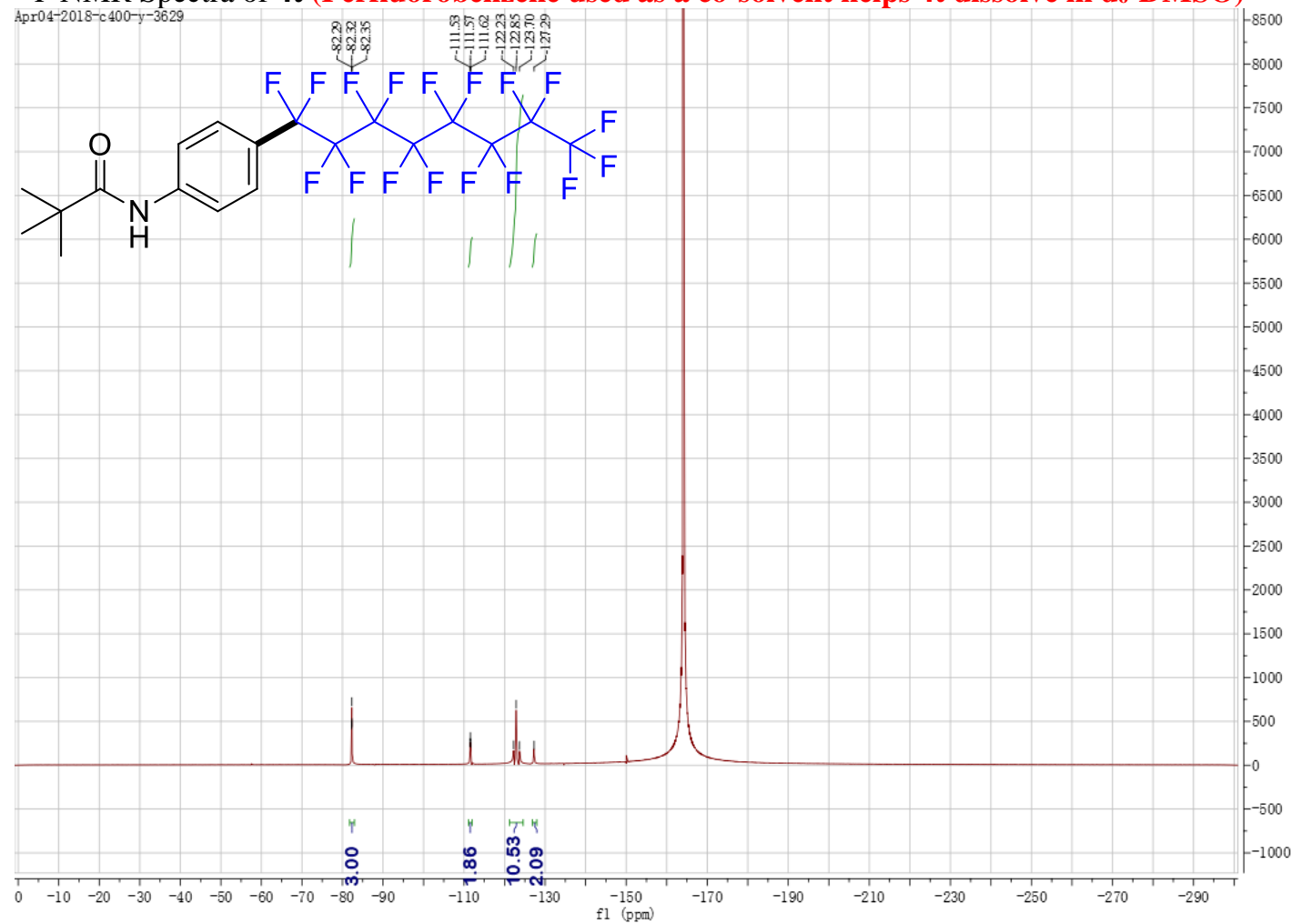

${ }^{13} \mathrm{C}$ NMR Spectra of $4 \mathrm{t}$ (Perfluorobenzene used as a co-solvent helps $4 \mathrm{t}$ dissolve in $\mathrm{d}_{6}$-DMSO)

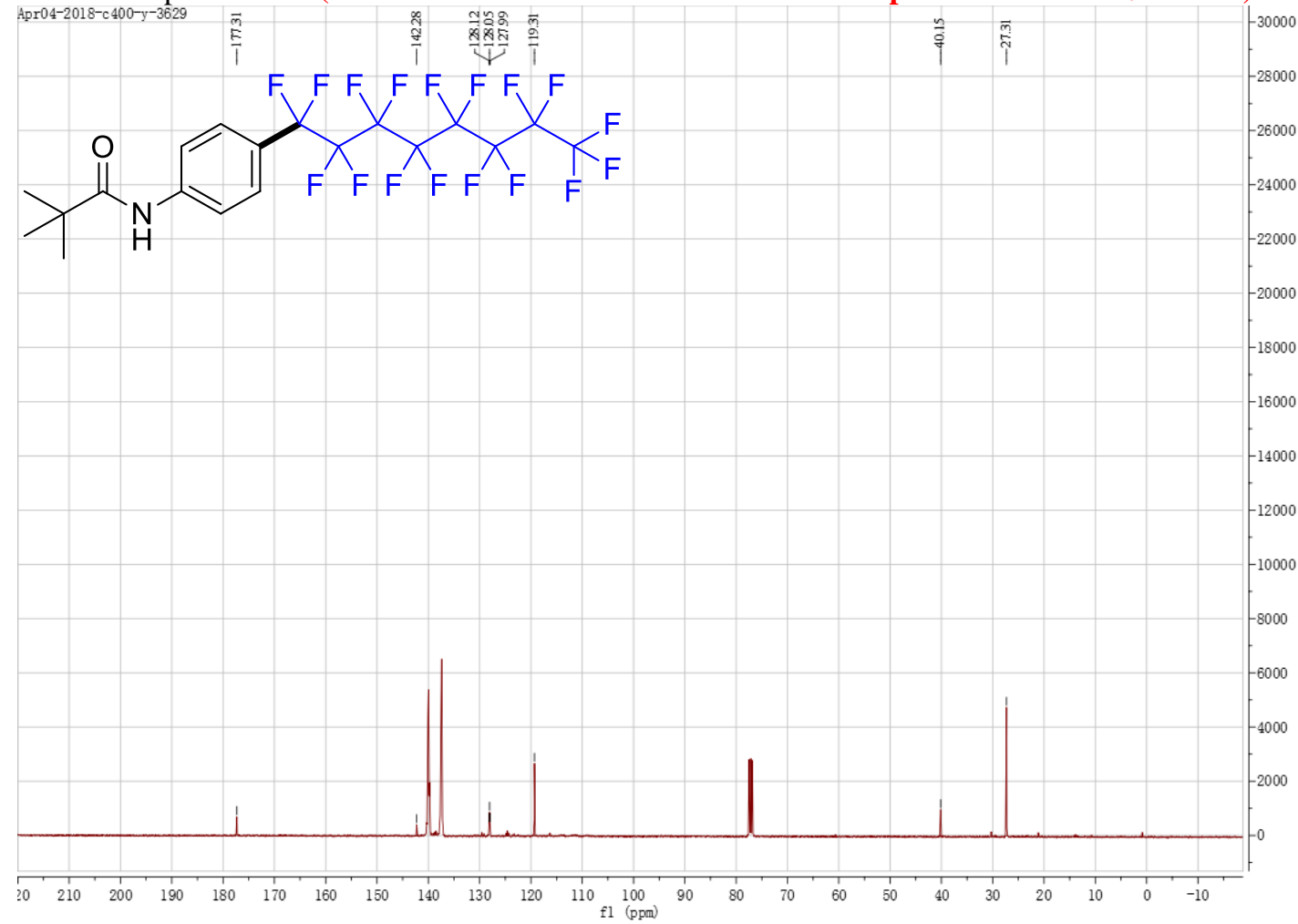


${ }^{1} \mathrm{H}$ NMR Spectra of $\mathbf{4 u}$ (Perfluorobenzene used as a co-solvent helps $4 \mathbf{u}$ dissolve in $\mathbf{d}_{6}$-DMSO)

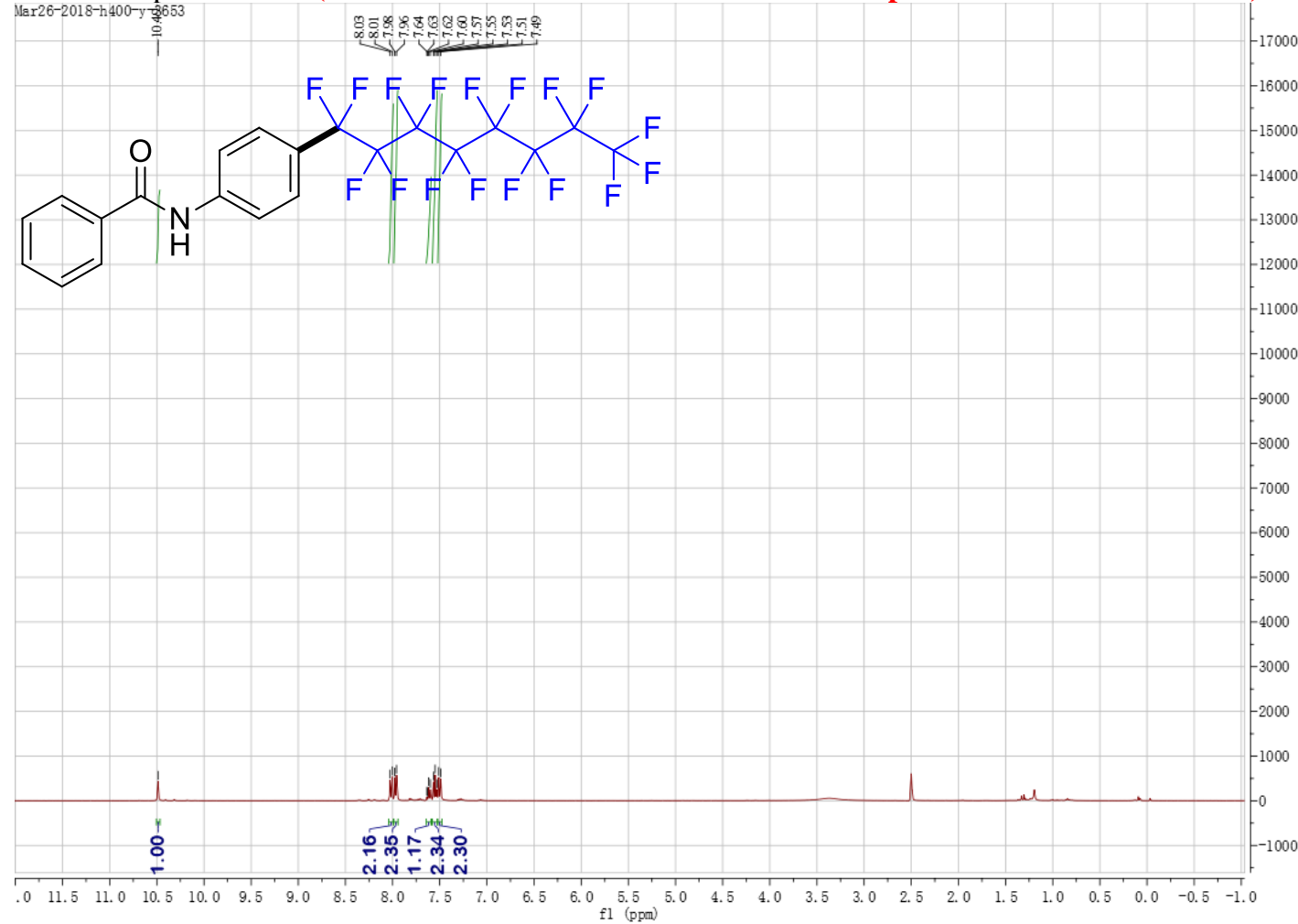

${ }^{19} \mathrm{~F}$ NMR Spectra of $\mathbf{4 u}$ (Perfluorobenzene used as a co-solvent helps $4 \mathbf{u}$ dissolve in $\mathbf{d}_{6}$-DMSO)

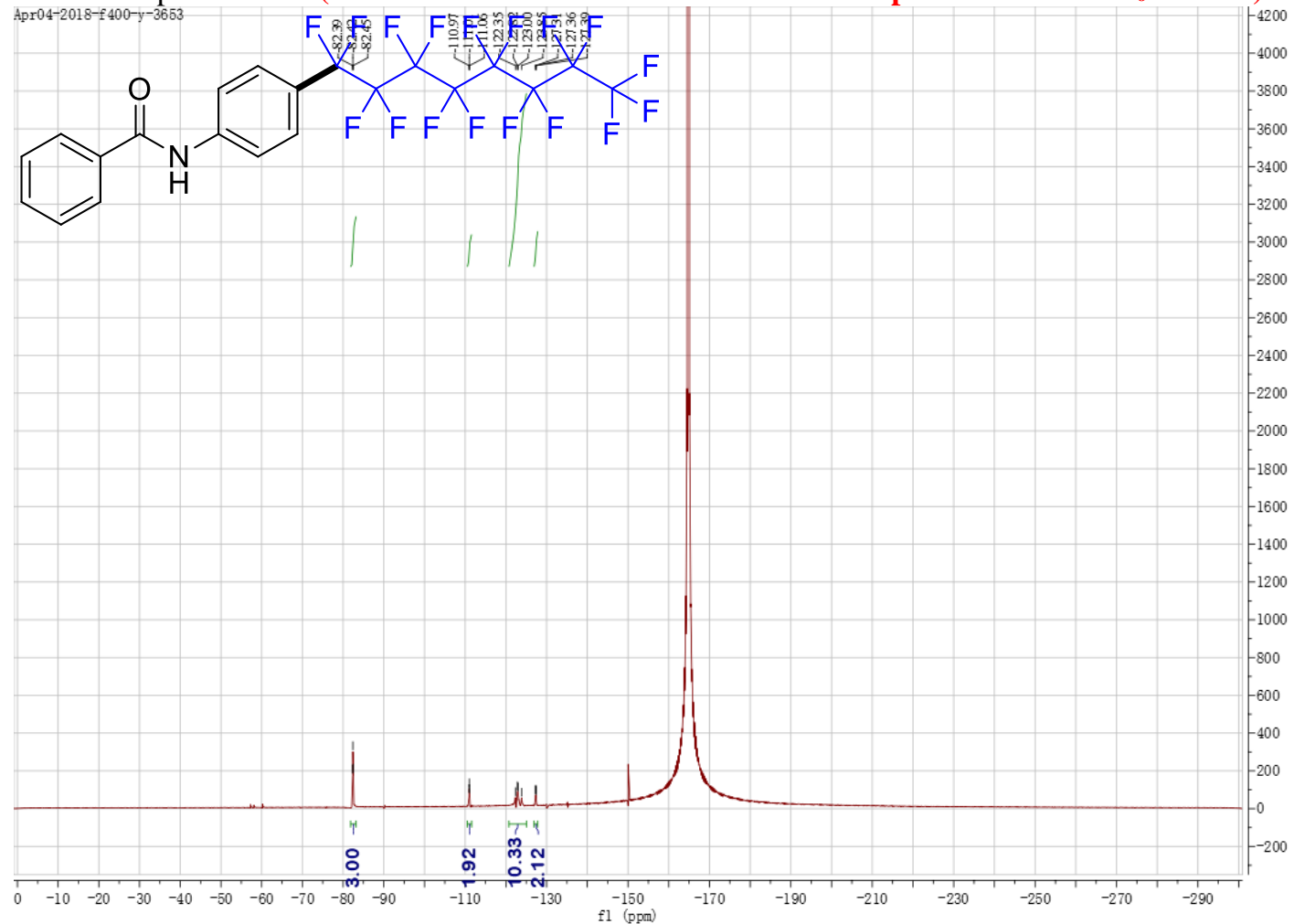


${ }^{13} \mathrm{C}$ NMR Spectra of $\mathbf{4 u}$ (Perfluorobenzene used as a co-solvent helps $4 \mathbf{u}$ dissolve in $\mathbf{d}_{6}$-DMSO)

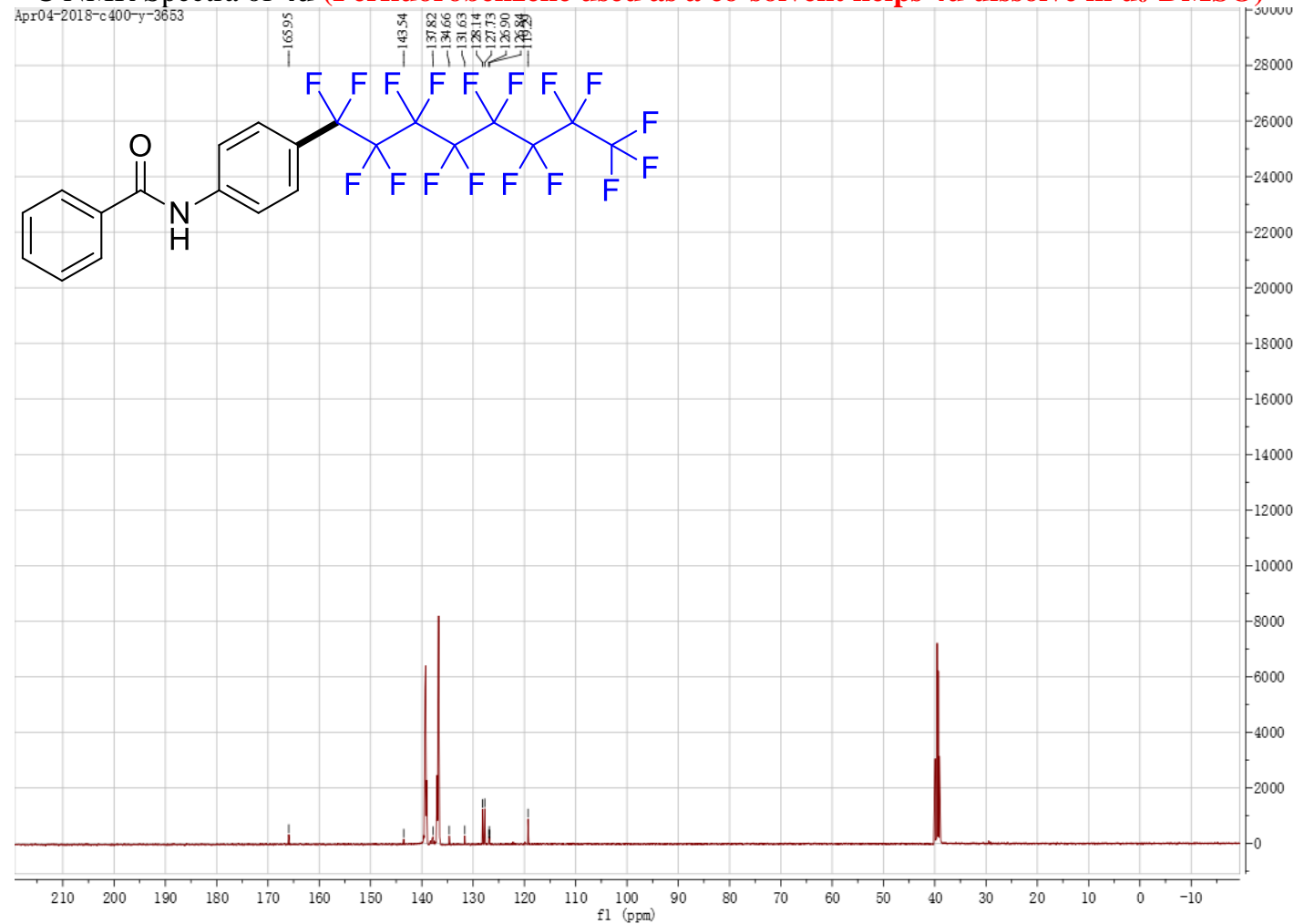

${ }^{1} \mathrm{H}$ NMR Spectra of $\mathbf{6 a}$

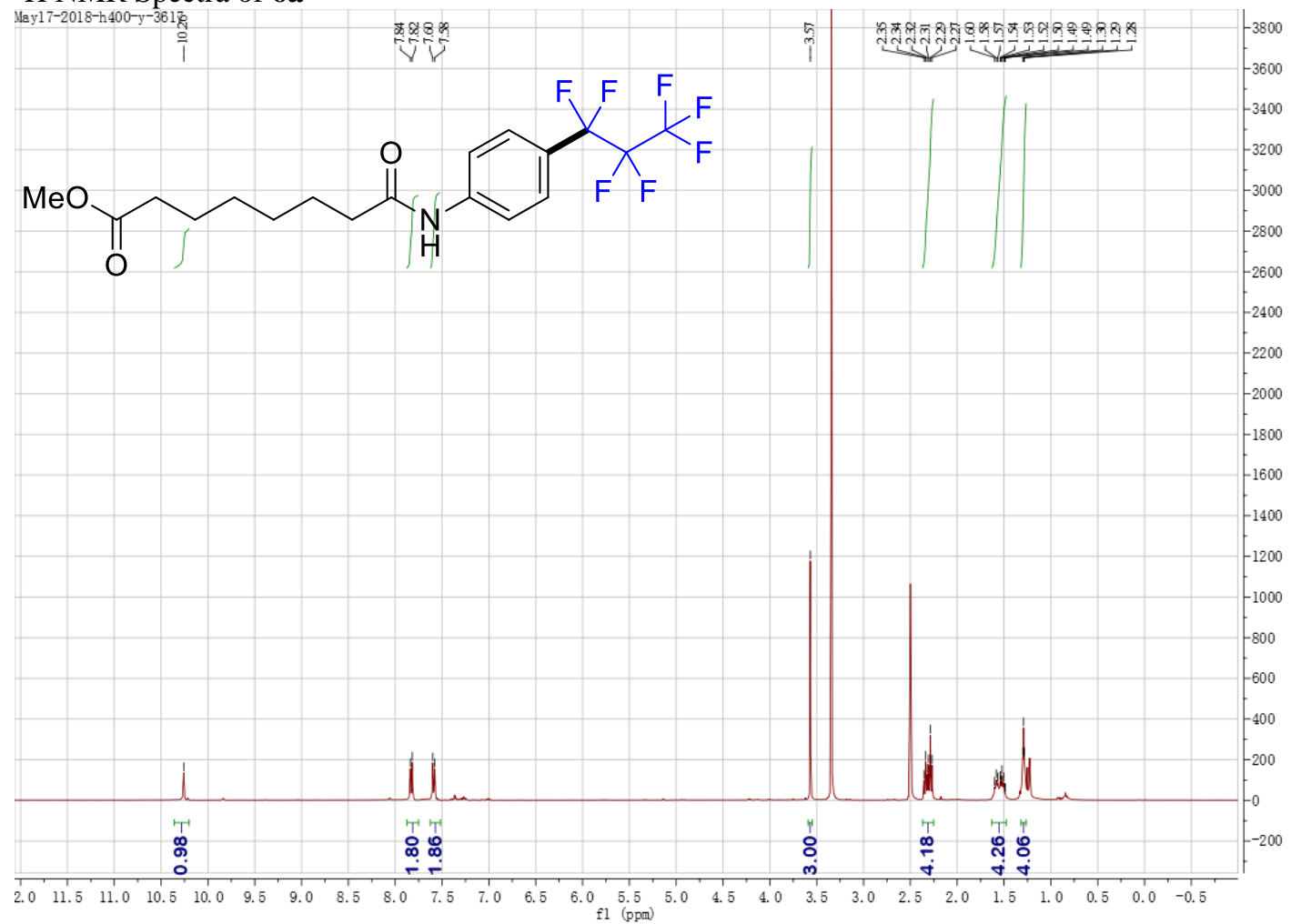


${ }^{19} \mathrm{~F}$ NMR Spectra of $\mathbf{6 a}$

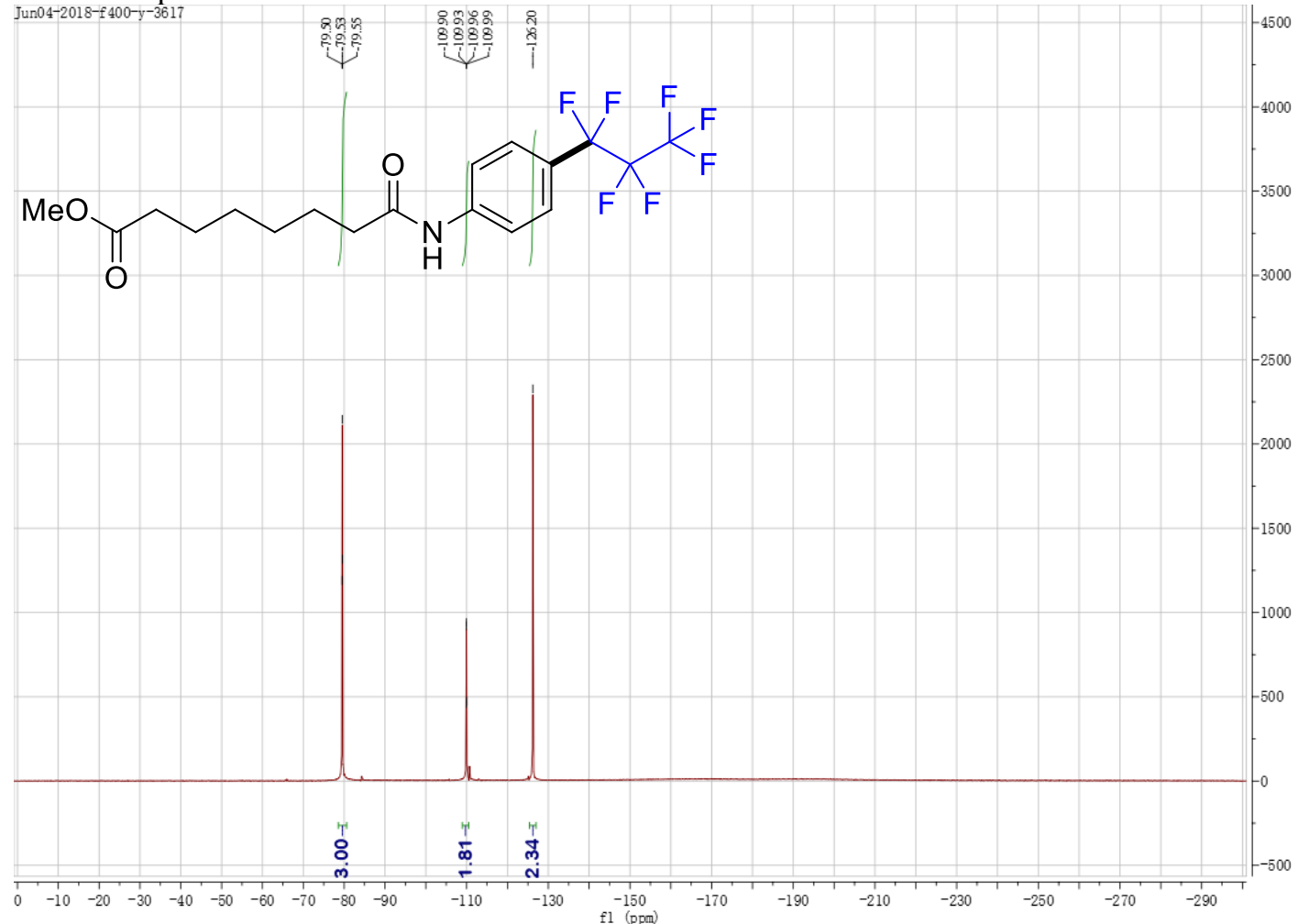

${ }^{13} \mathrm{C}$ NMR Spectra of $\mathbf{6 a}$

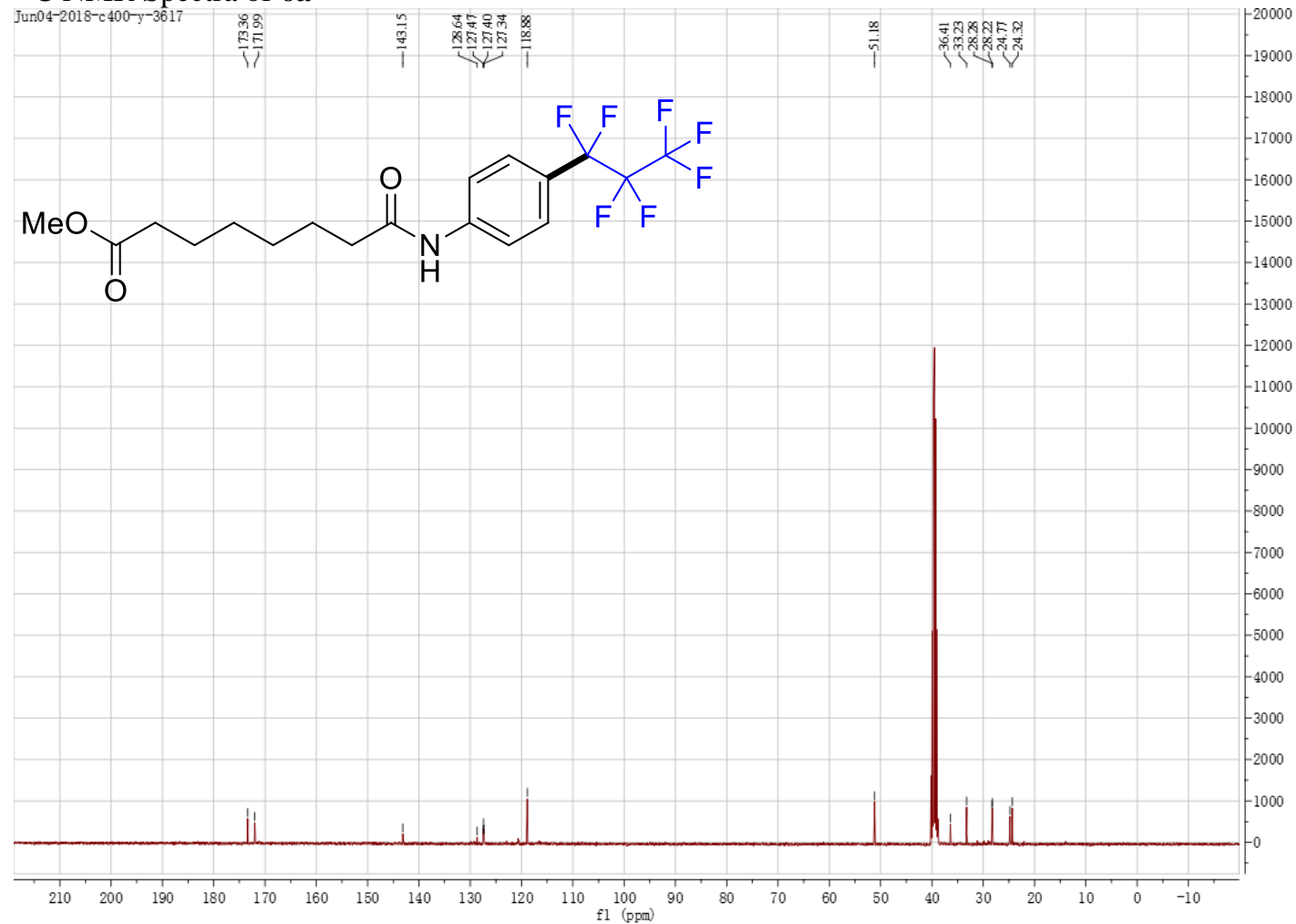


${ }^{1} \mathrm{H}$ NMR Spectra of $\mathbf{6 b}$

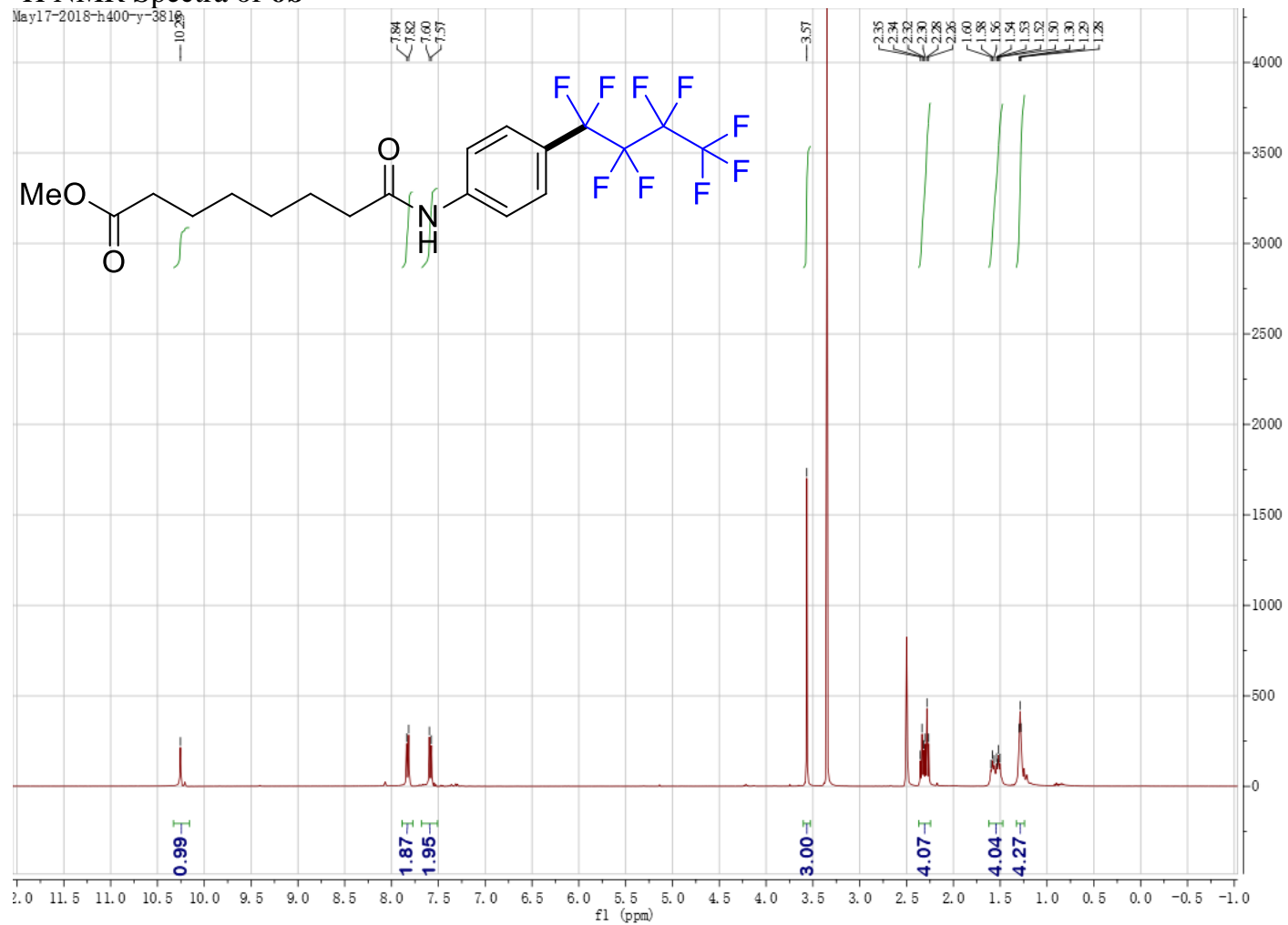

${ }^{19}$ F NMR Spectra of $6 \mathbf{b}$

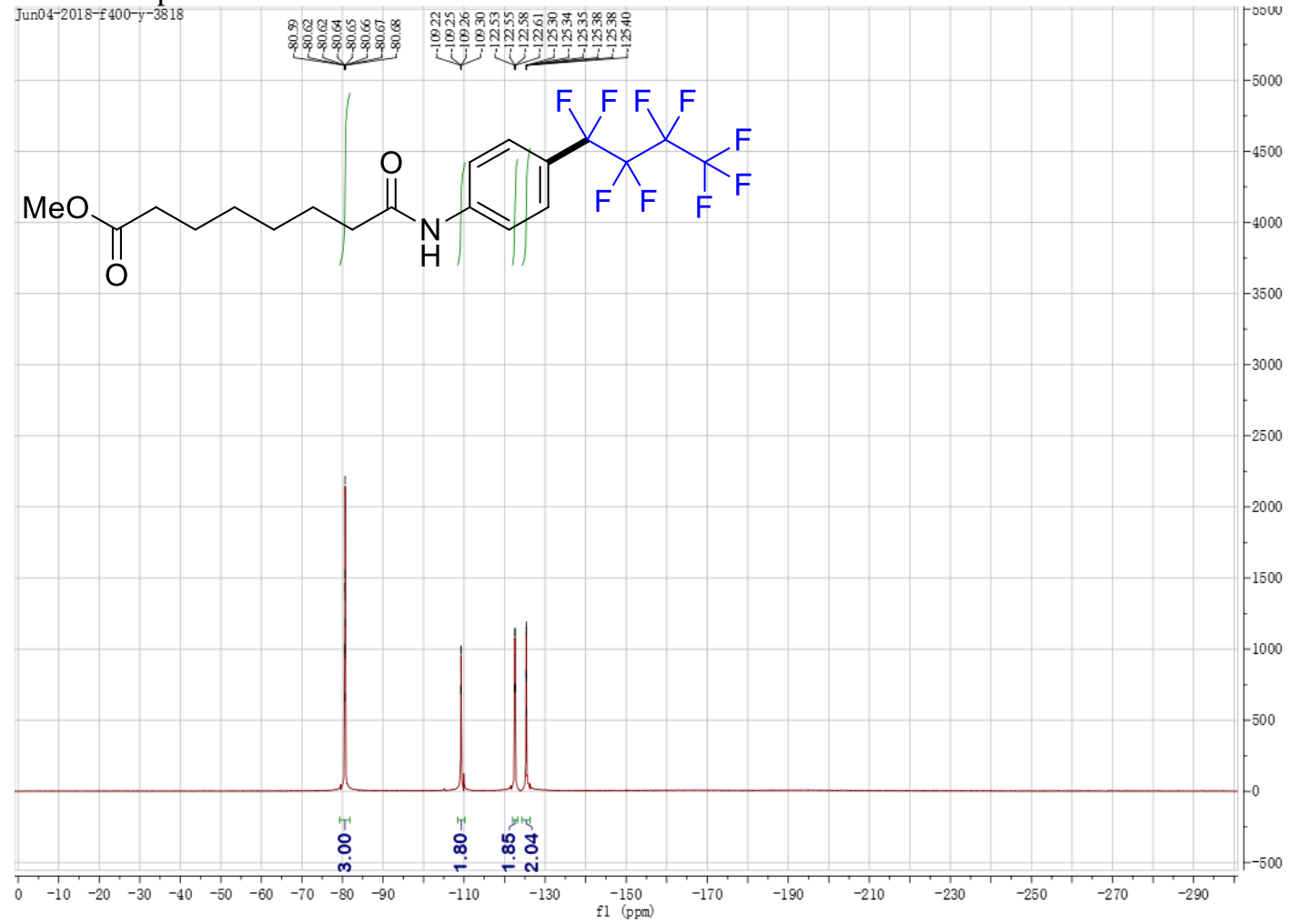


${ }^{13}$ C NMR Spectra of $\mathbf{6 b}$

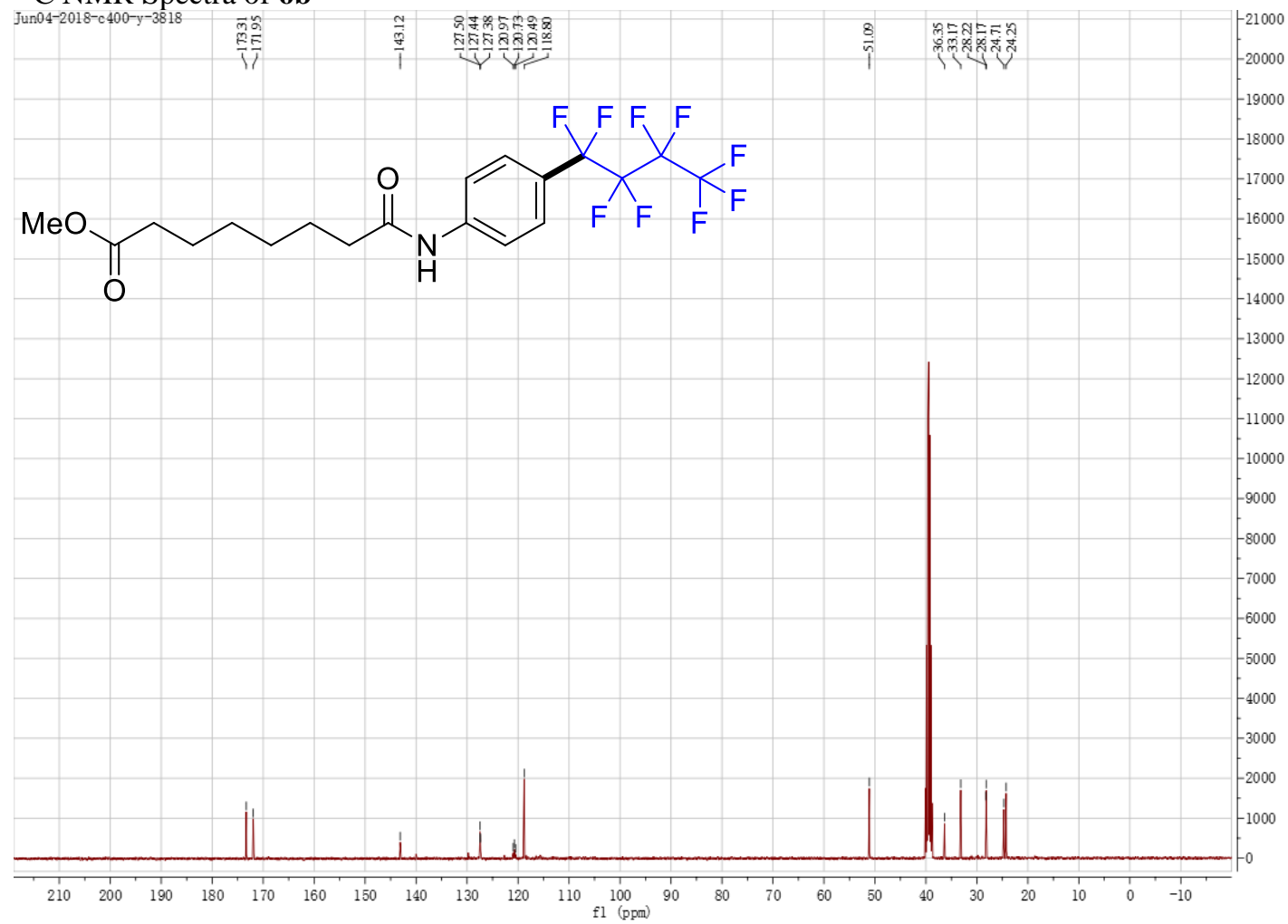

${ }^{1} \mathrm{H}$ NMR Spectra of $\mathbf{6 c}$

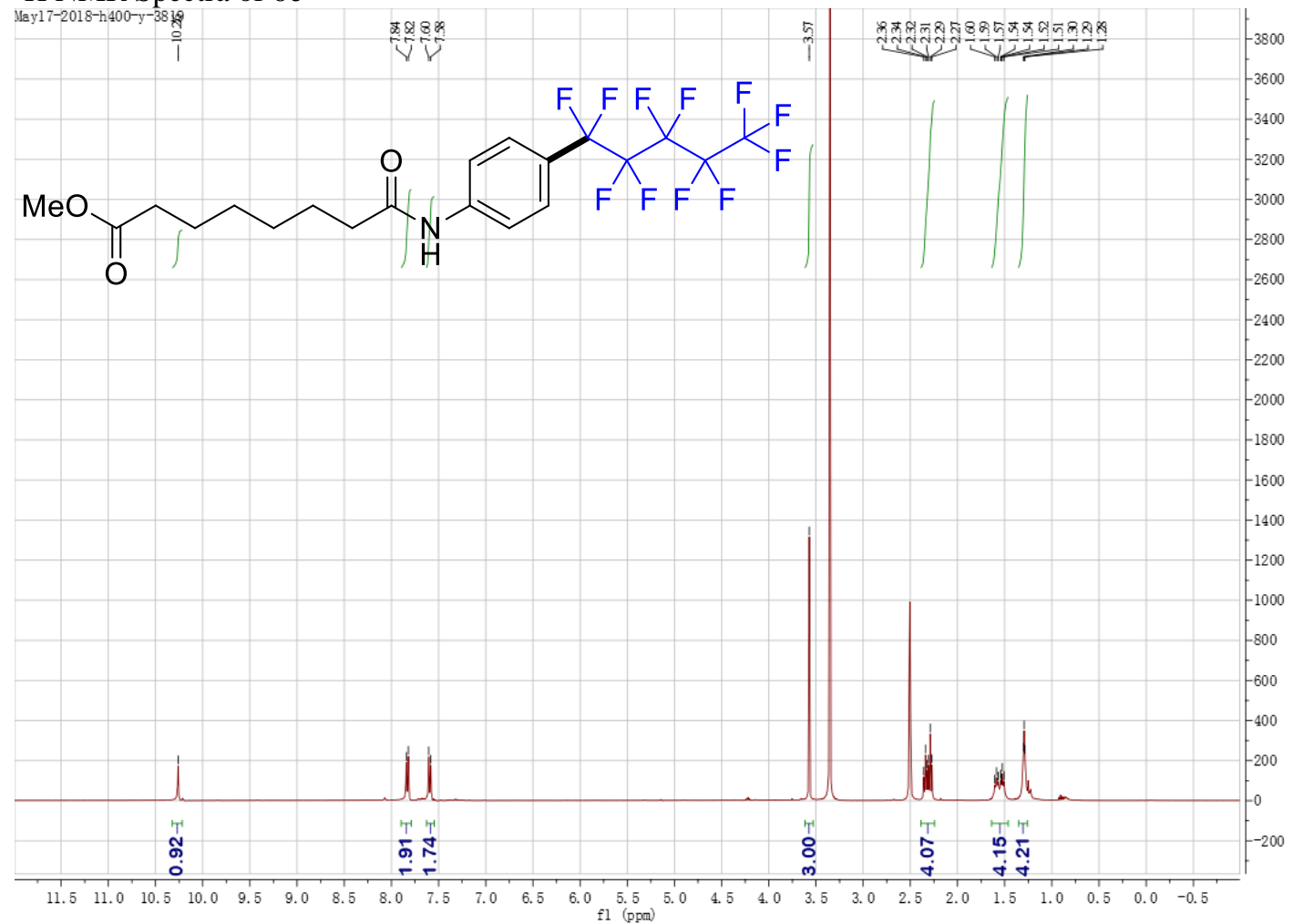


${ }^{19}$ F NMR Spectra of $\mathbf{6 c}$

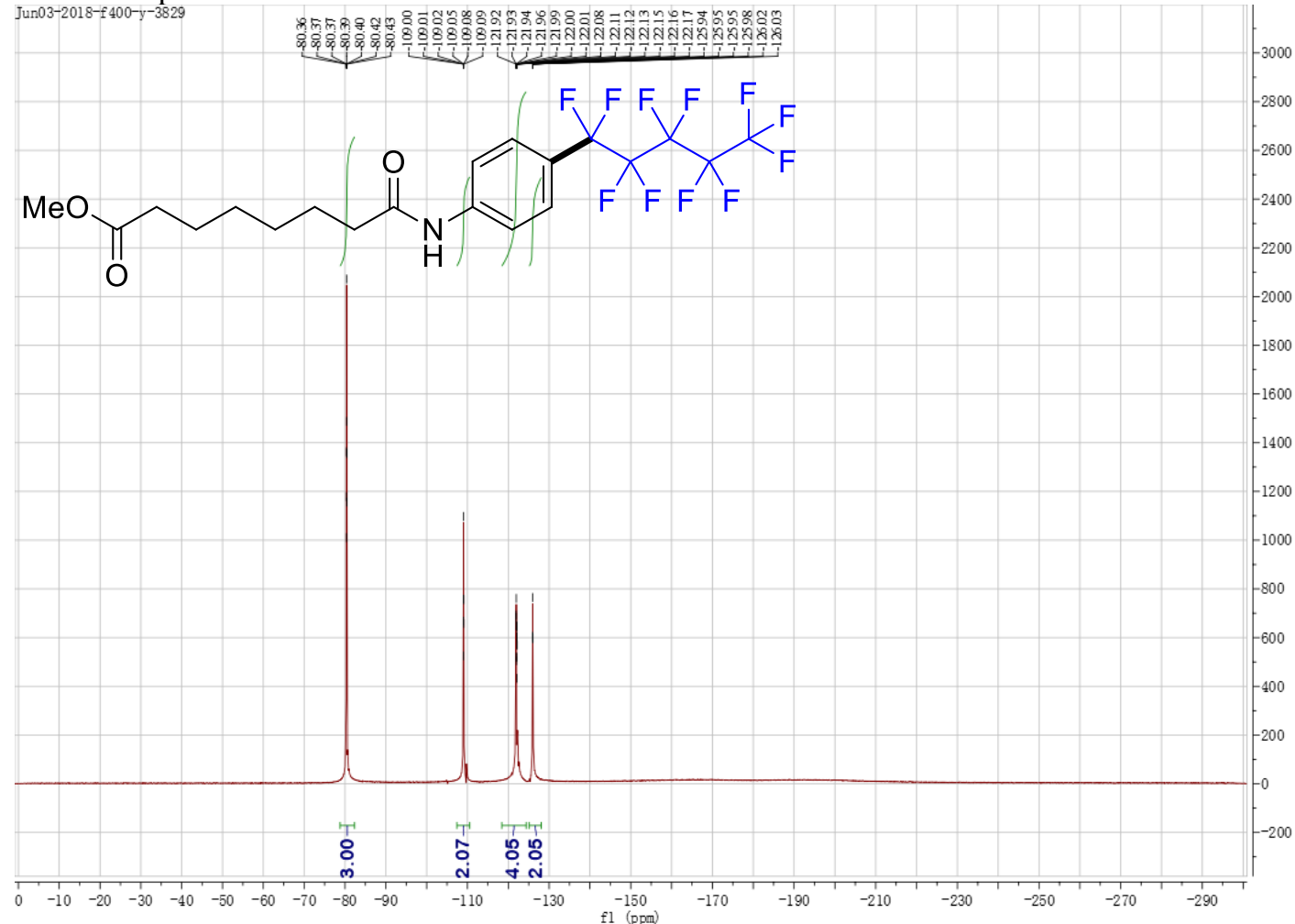

${ }^{13} \mathrm{C}$ NMR Spectra of $\mathbf{6 c}$

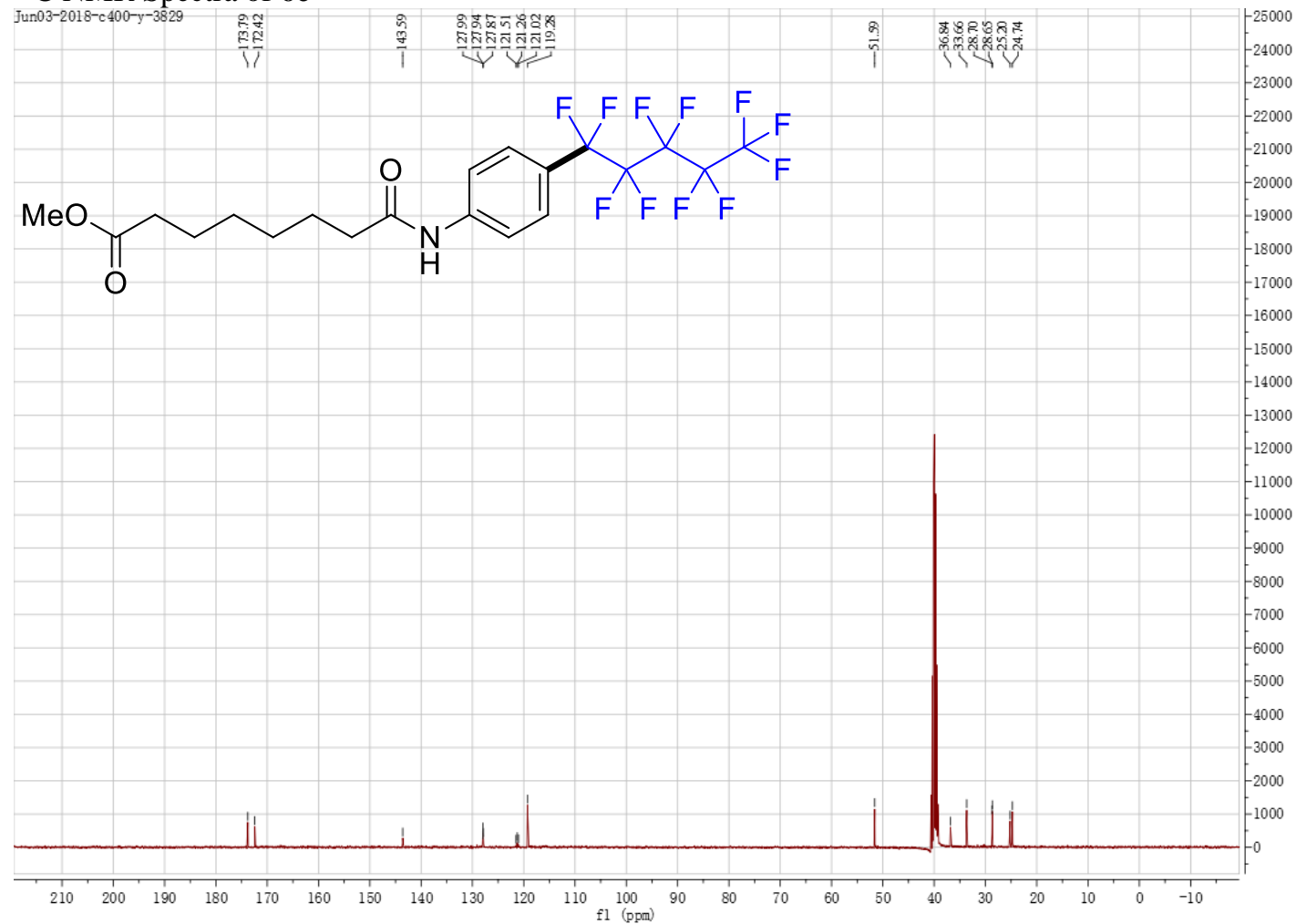


${ }^{1} \mathrm{H}$ NMR Spectra of $\mathbf{6 d}$

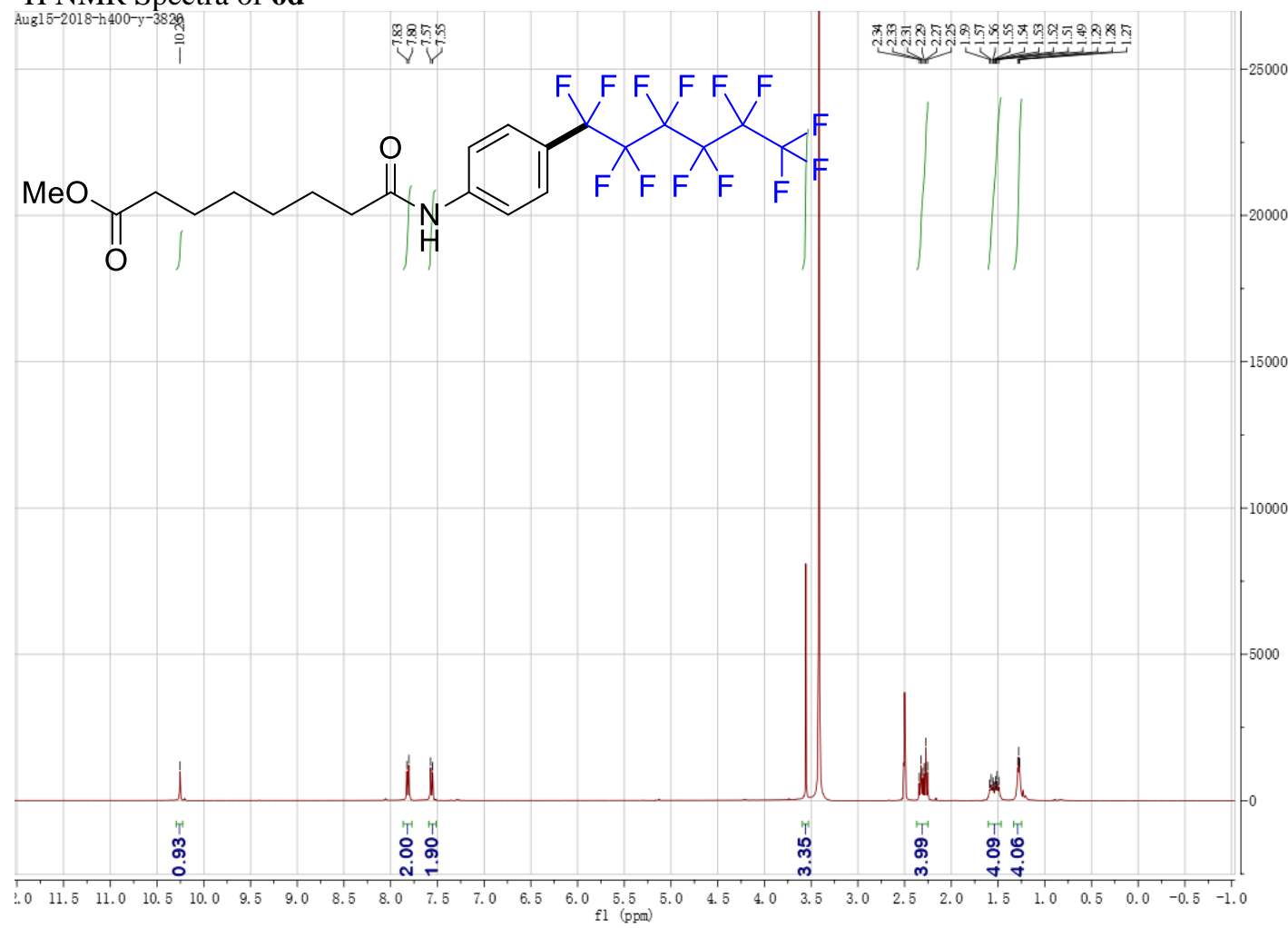

${ }^{19}$ F NMR Spectra of $\mathbf{6 d}$

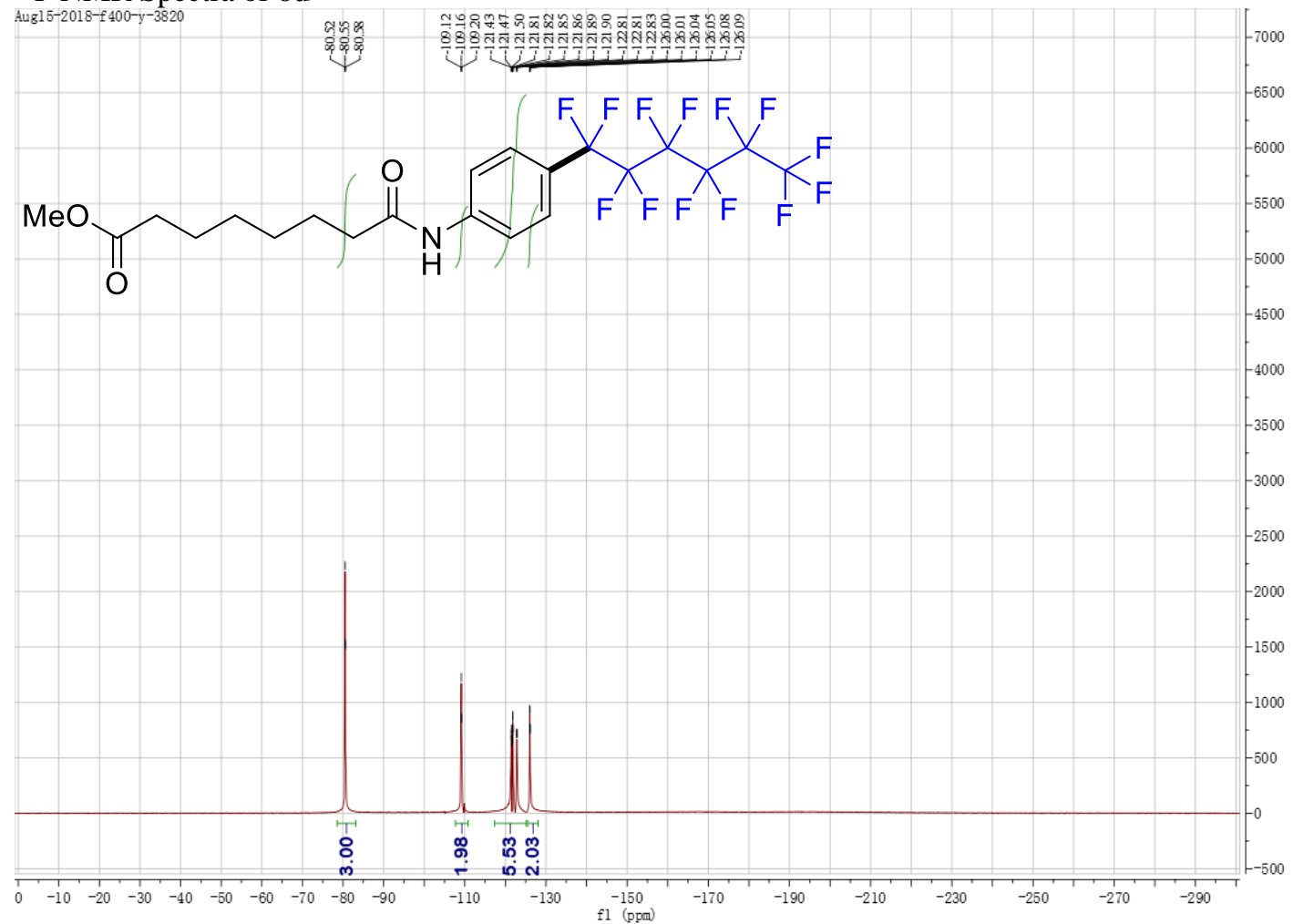




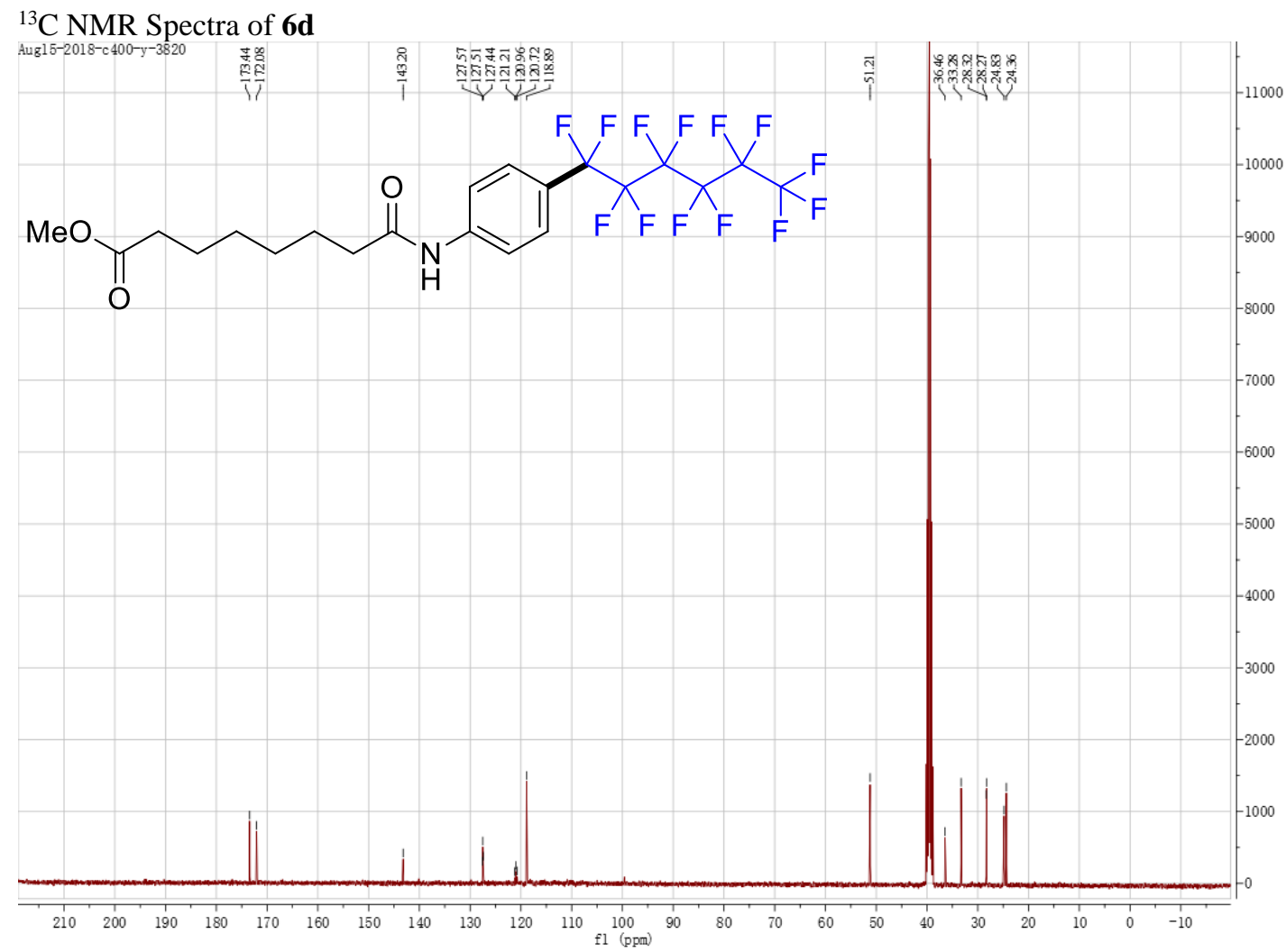

${ }^{1} \mathrm{H}$ NMR Spectra of $6 \mathrm{e}$ (Perfluorobenzene used as a co-solvent helps 6e dissolve in d6-DMSO)

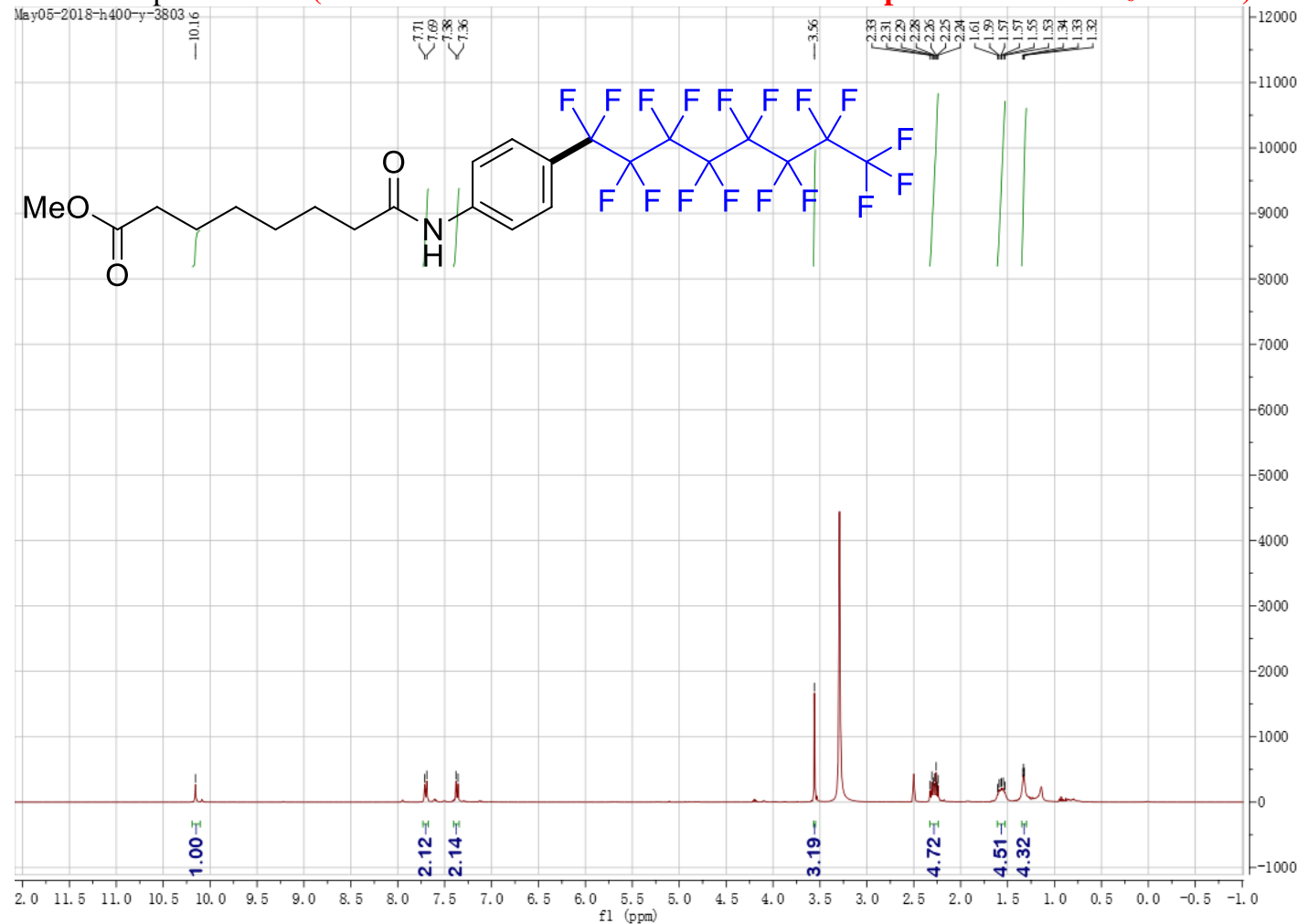


${ }^{19}$ F NMR Spectra of 6 e (Perfluorobenzene used as a co-solvent helps 6 e dissolve in $\mathrm{d}_{6}$-DMSO)

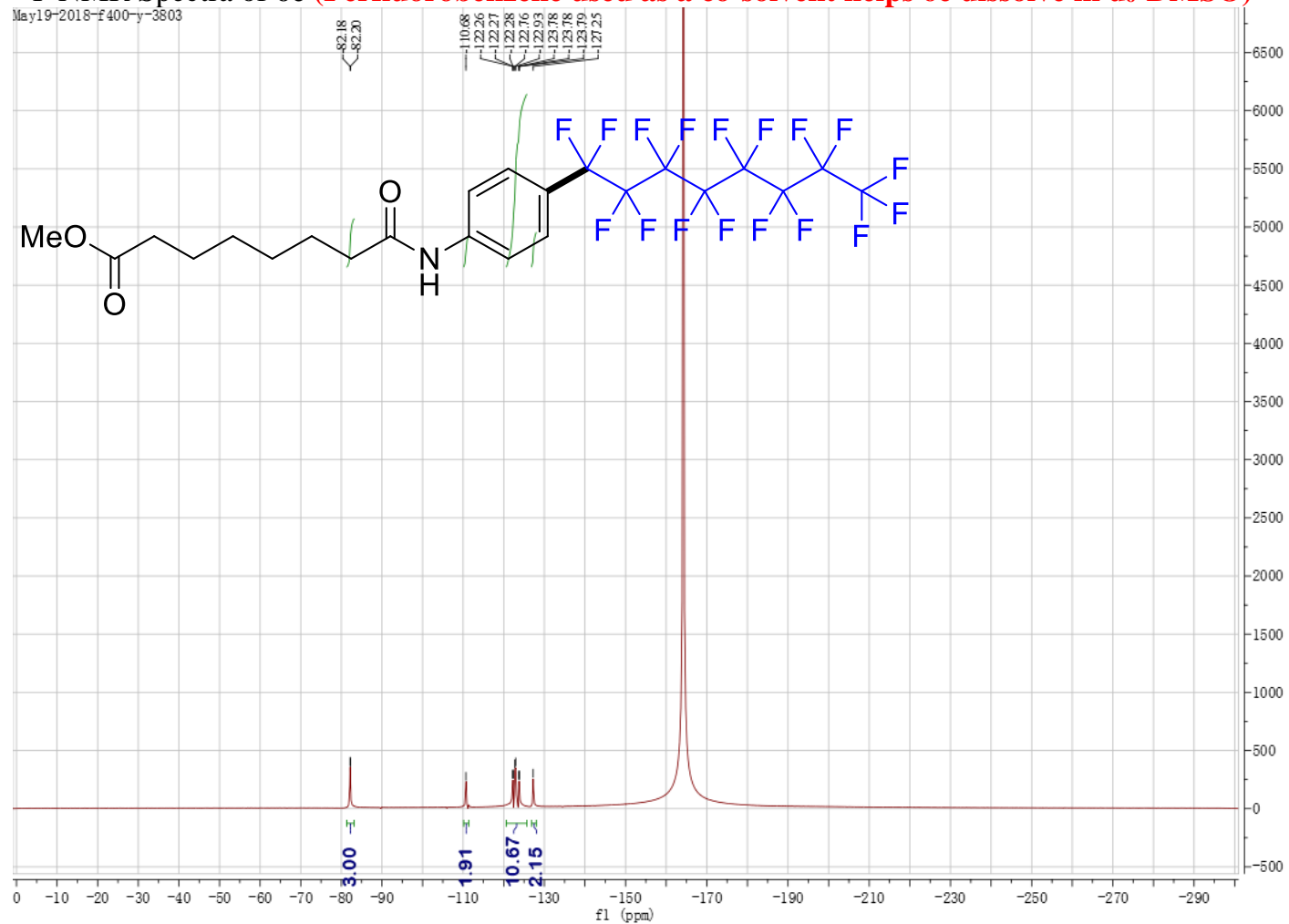

${ }^{13} \mathrm{C}$ NMR Spectra of $6 \mathrm{e}$ (Perfluorobenzene used as a co-solvent helps 6e dissolve in d6-DMSO)

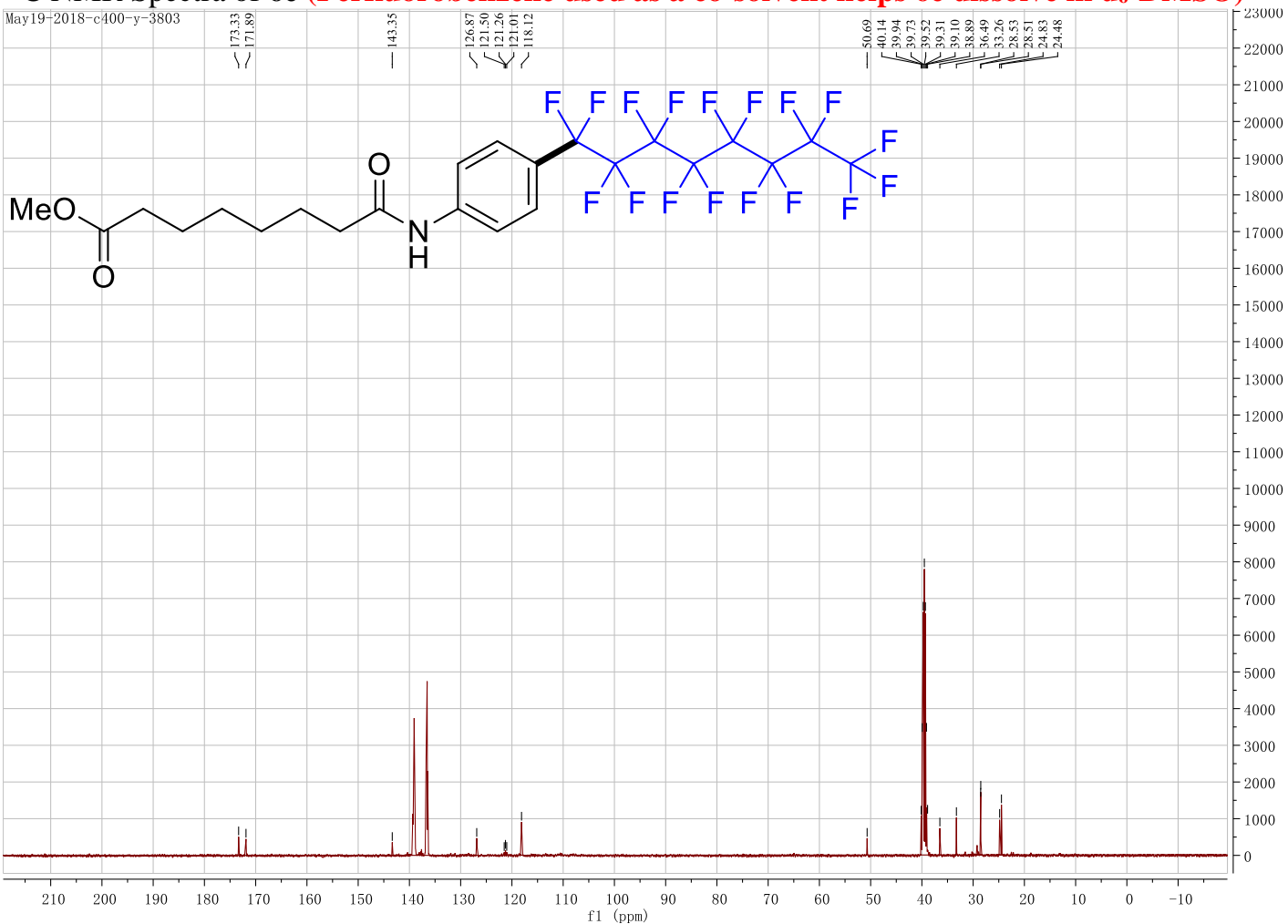


${ }^{1} \mathrm{H}$ NMR Spectra of $6 \mathrm{f}$ (Perfluorobenzene used as a co-solvent helps 6 f dissolve in d6-DMSO)

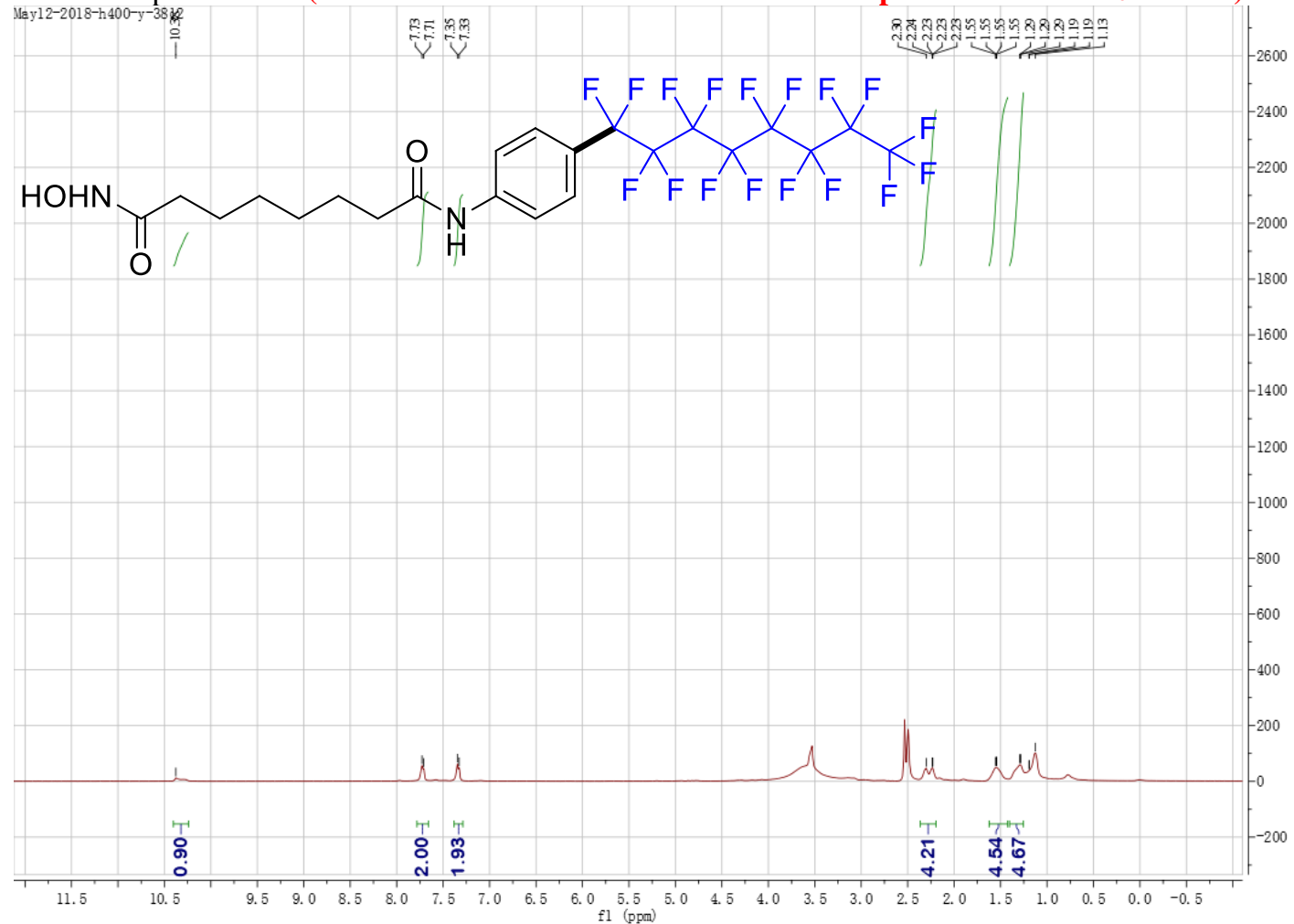

${ }^{19} \mathrm{~F}$ NMR Spectra of $6 \mathbf{f}$ (Perfluorobenzene used as a co-solvent helps 6 f dissolve in $\mathbf{d}_{6}$-DMSO)

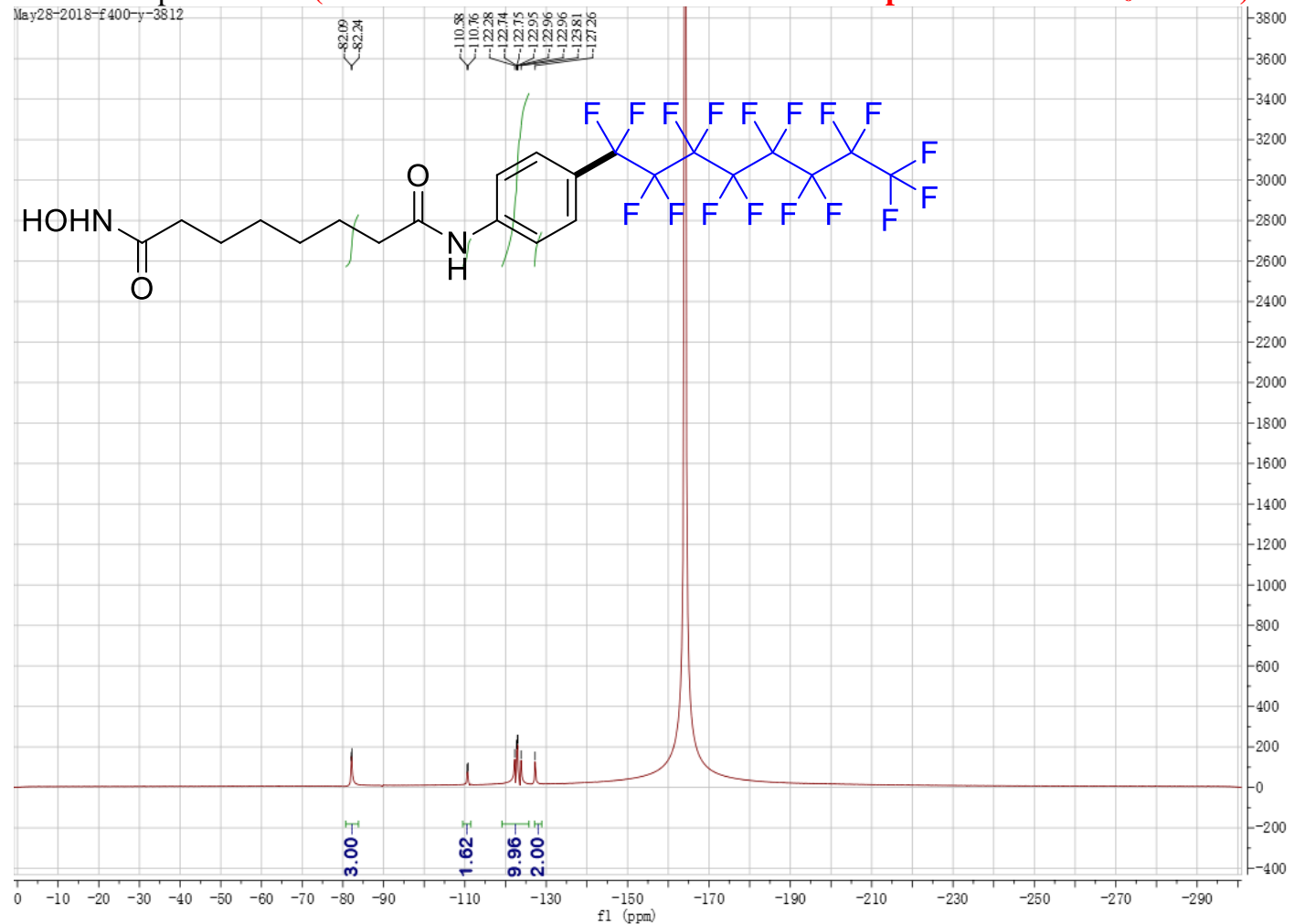


${ }^{13} \mathrm{C}$ NMR Spectra of $6 \mathrm{f}$ (Perfluorobenzene used as a co-solvent helps 6 f dissolve in $\mathrm{d}_{6}$-DMSO)

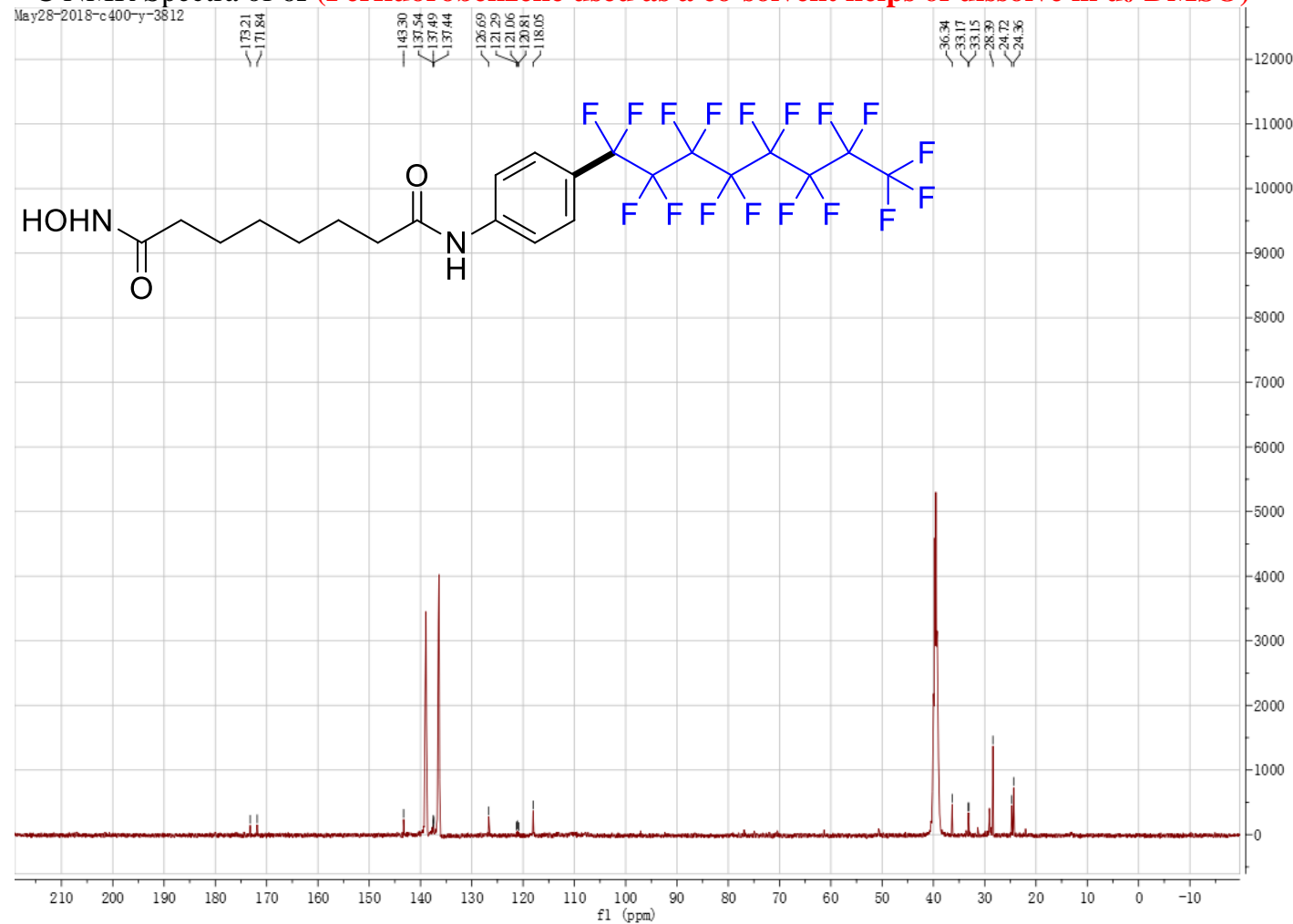

${ }^{1} \mathrm{H}$ NMR Spectra of $\mathbf{7 b}$

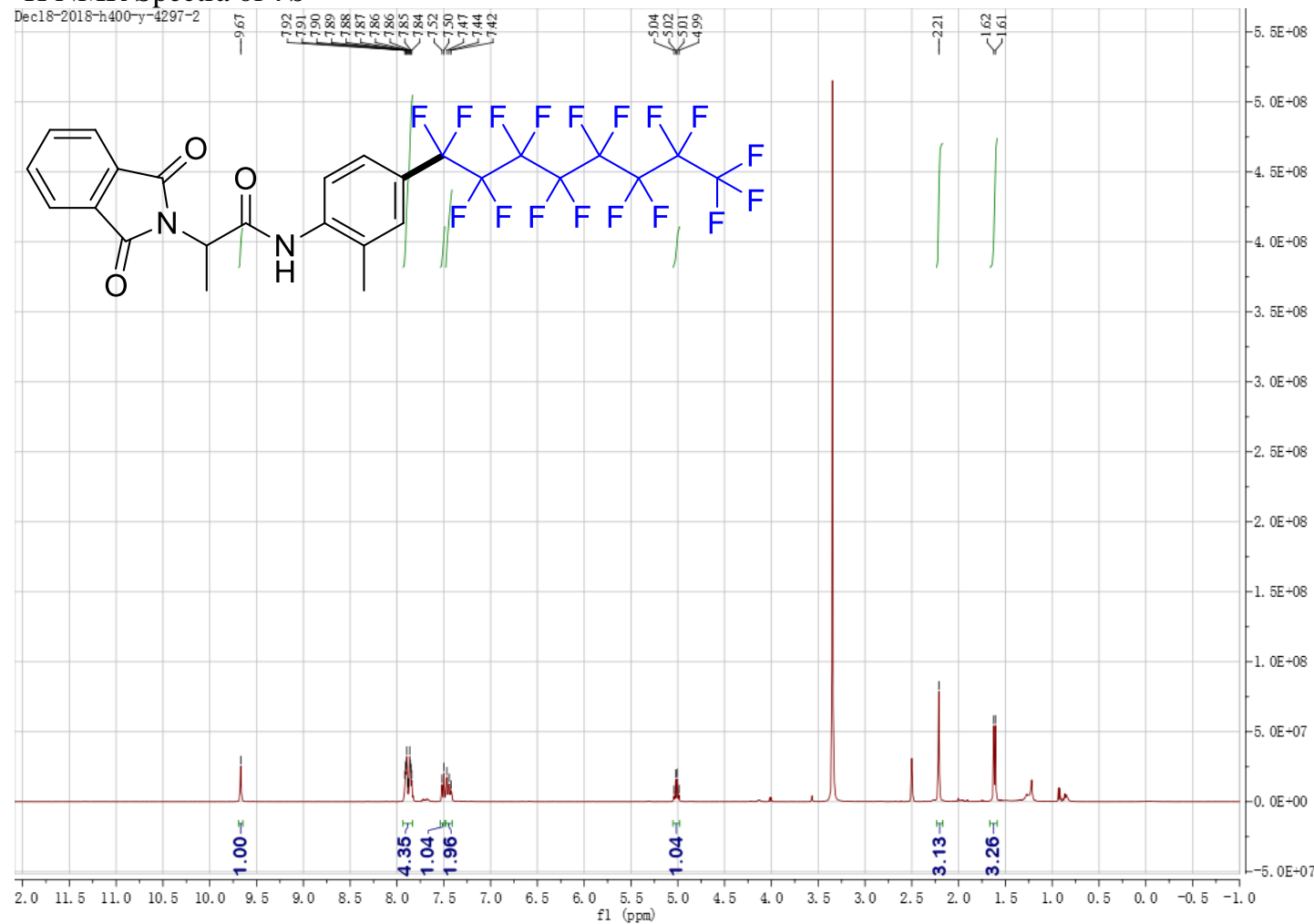


${ }^{19} \mathrm{~F}$ NMR Spectra of $\mathbf{7 b}$

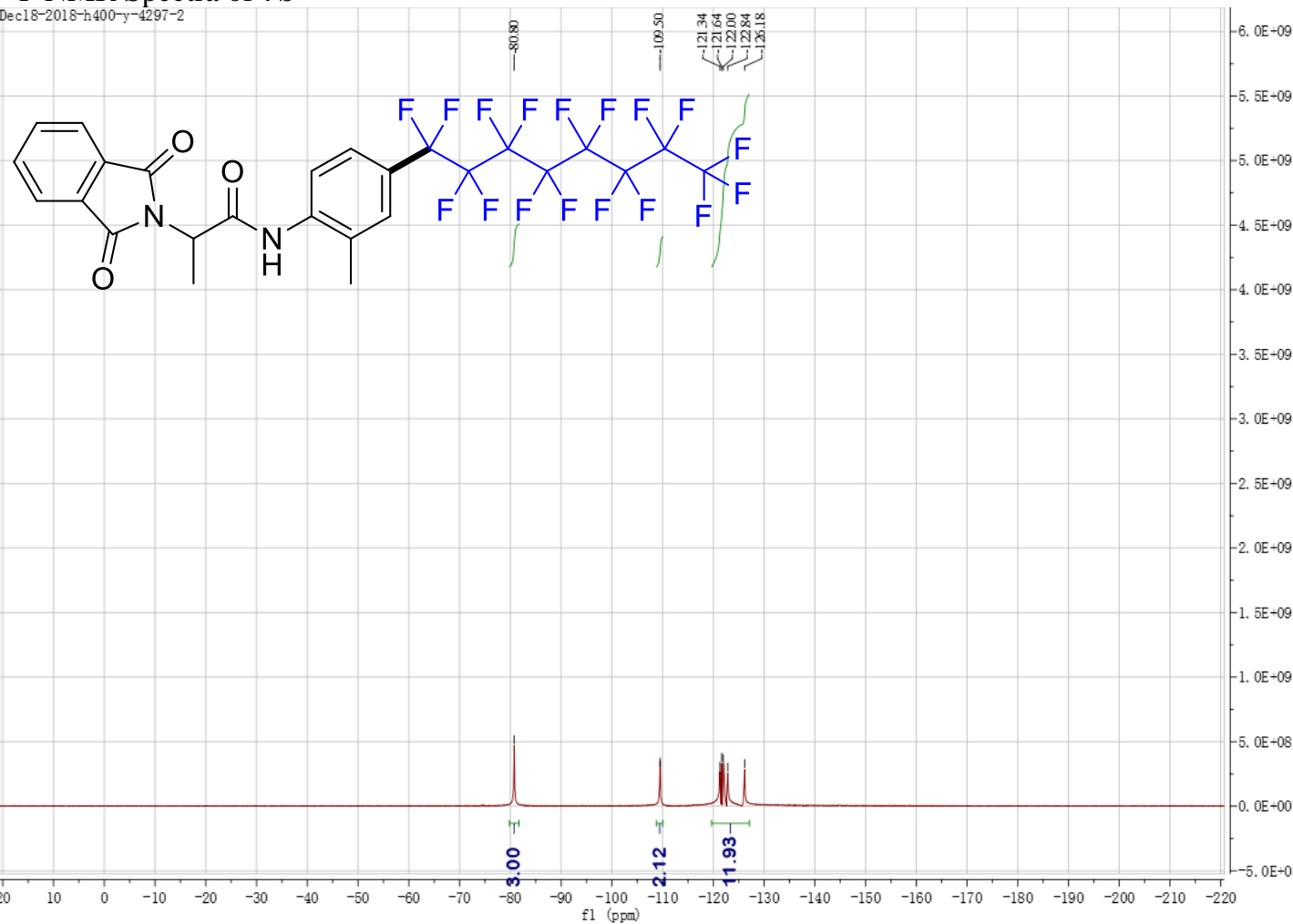

${ }^{13} \mathrm{C}$ NMR Spectra of $\mathbf{7 b}$

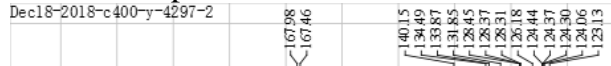
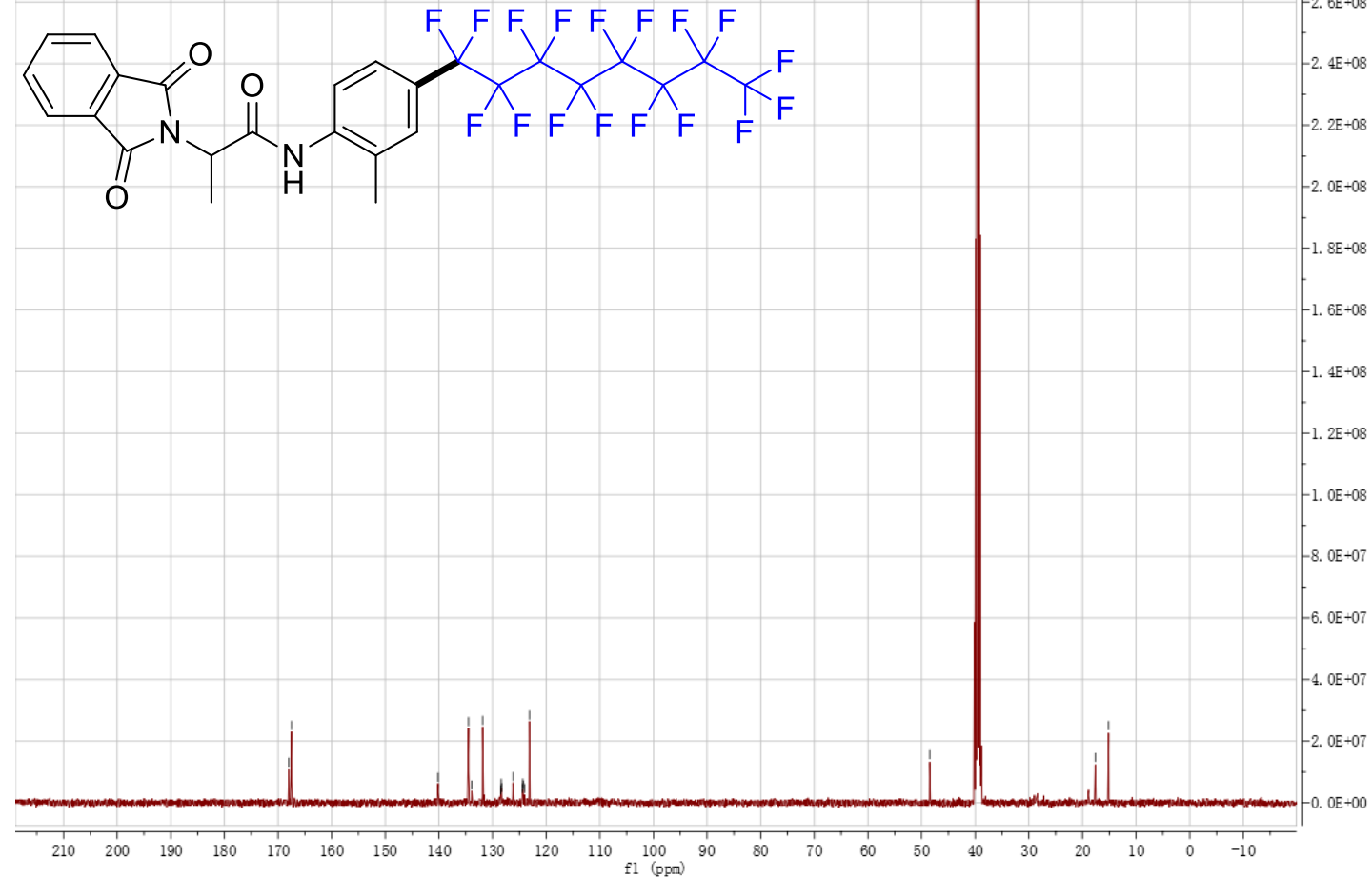
${ }^{1} \mathrm{H}$ NMR Spectra of $\mathbf{8}$

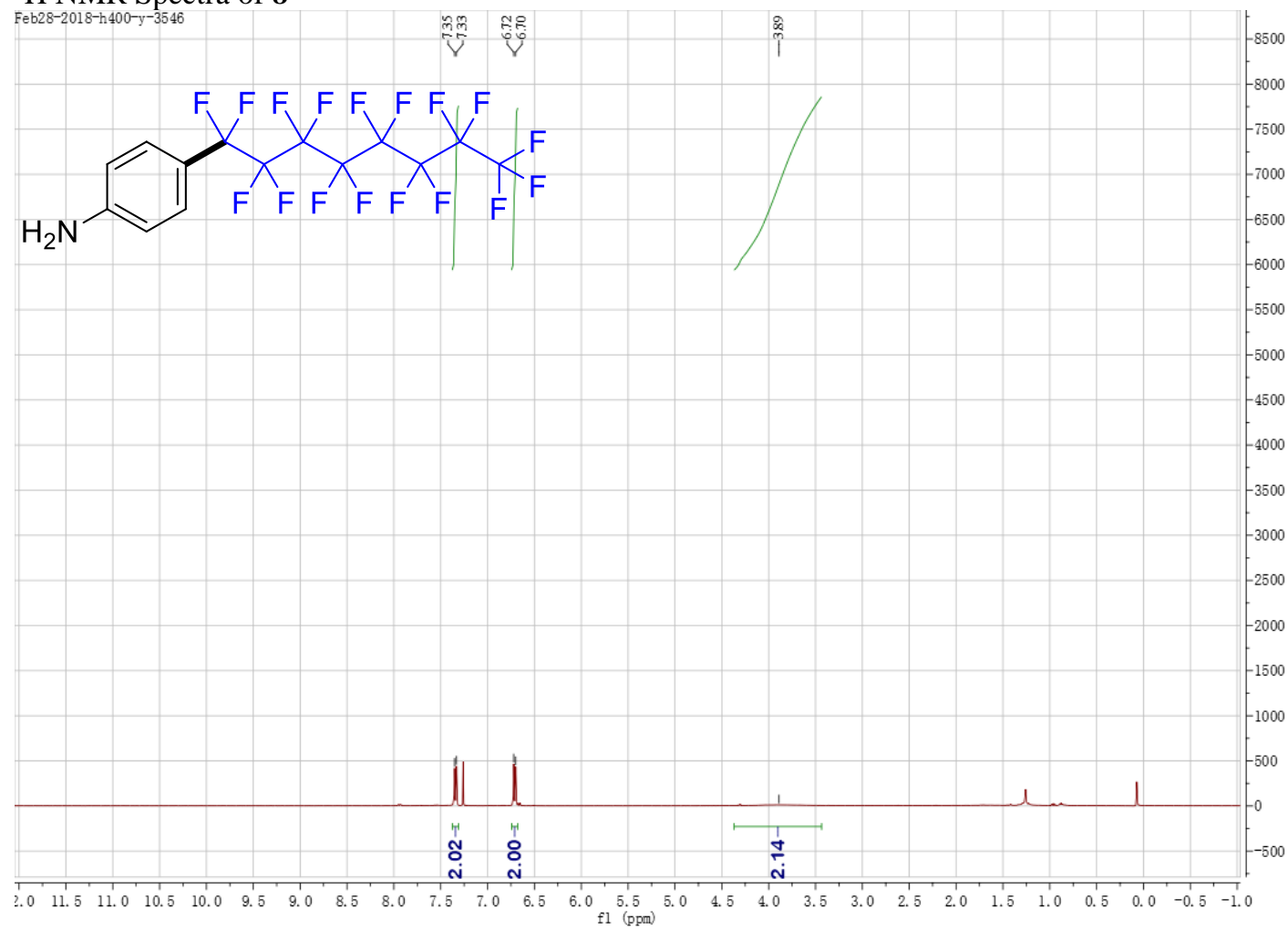

${ }^{19} \mathrm{~F}$ NMR Spectra of $\mathbf{8}$ Nar12-2018-f 400-y-3546

ER\&

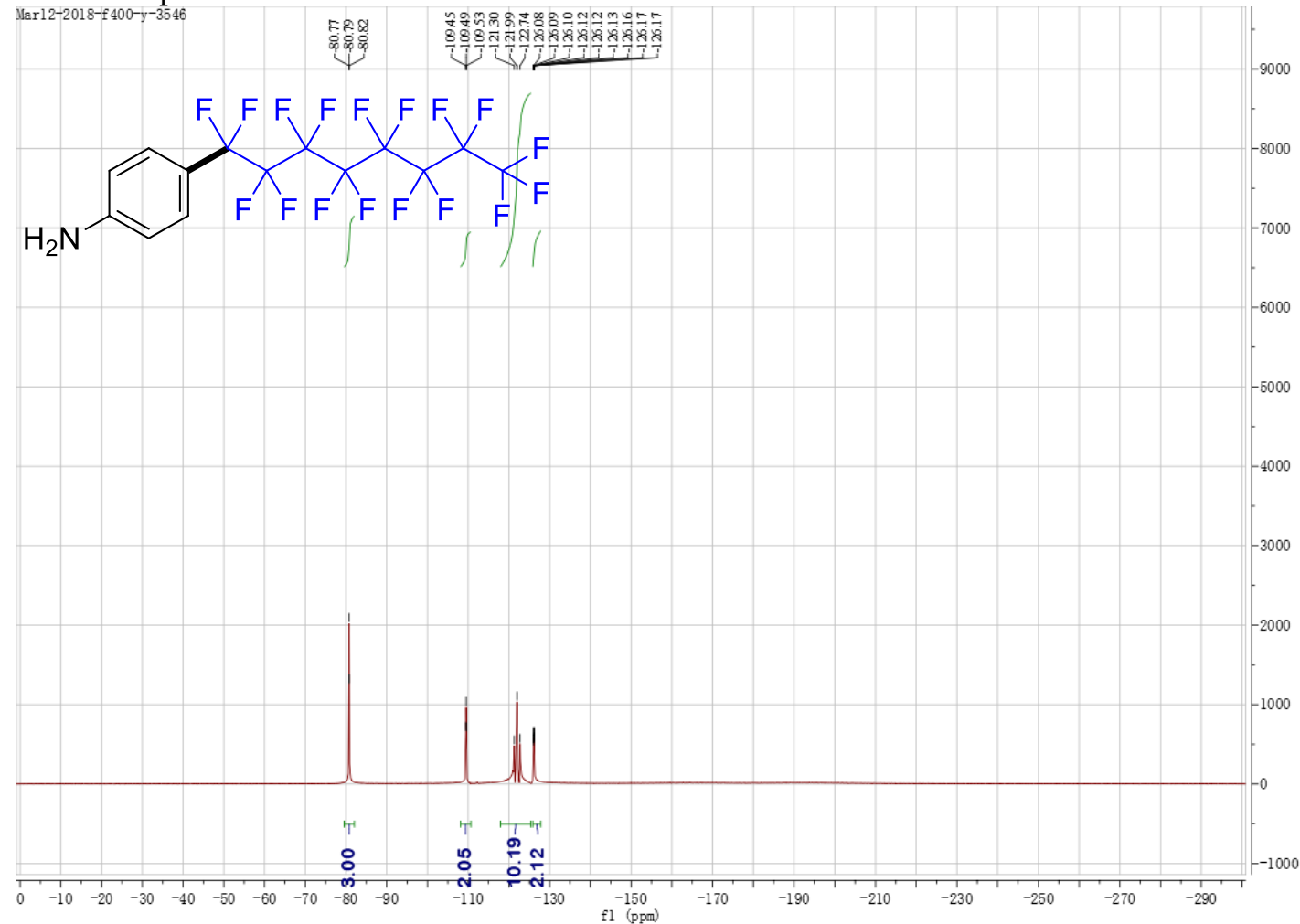


${ }^{13} \mathrm{C}$ NMR Spectra of 8

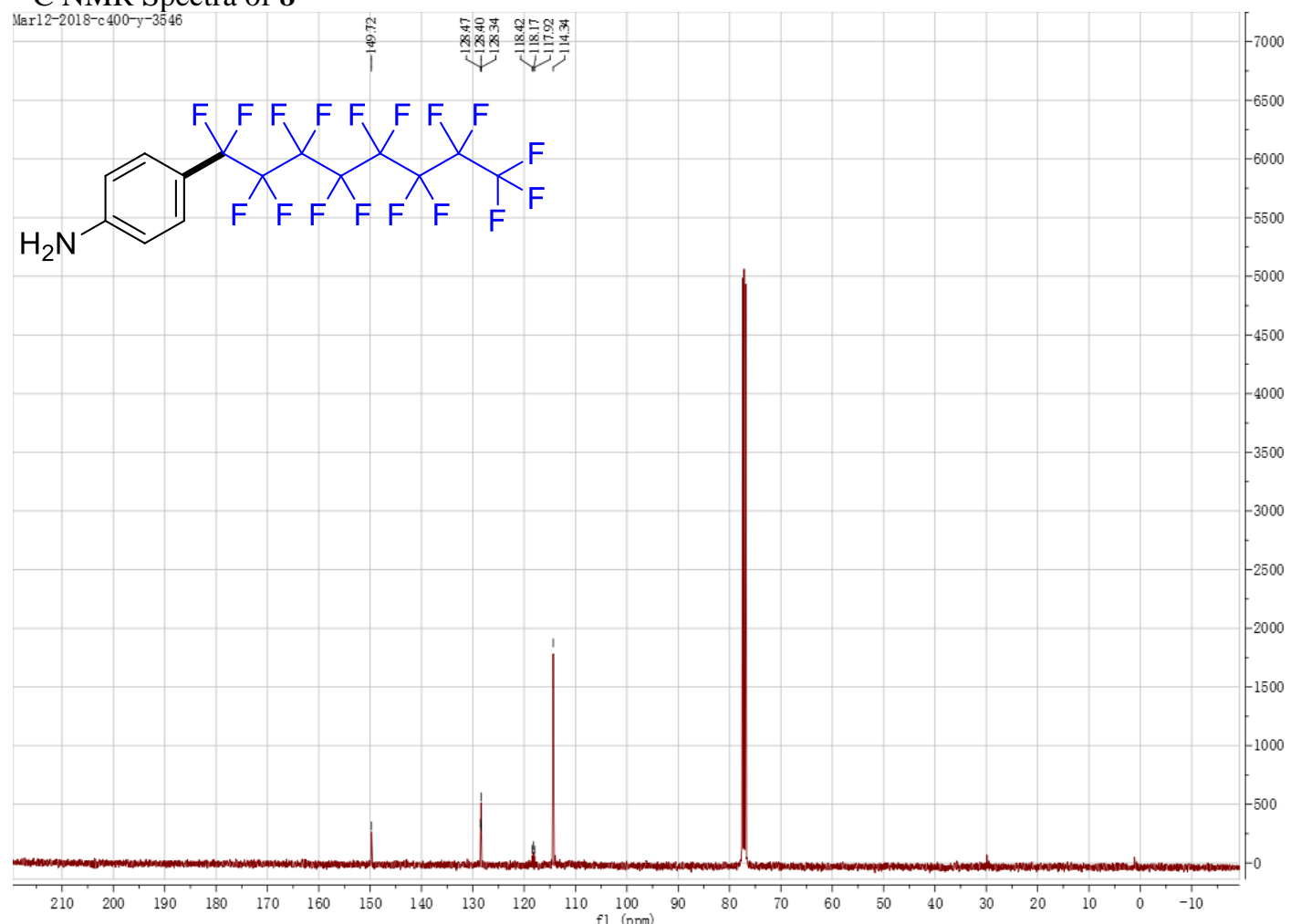

${ }^{1} \mathrm{H}$ NMR, ${ }^{19} \mathrm{~F}$ NMR \& ${ }^{13} \mathrm{C}$ NMR Spectra of $\mathbf{1 0}$

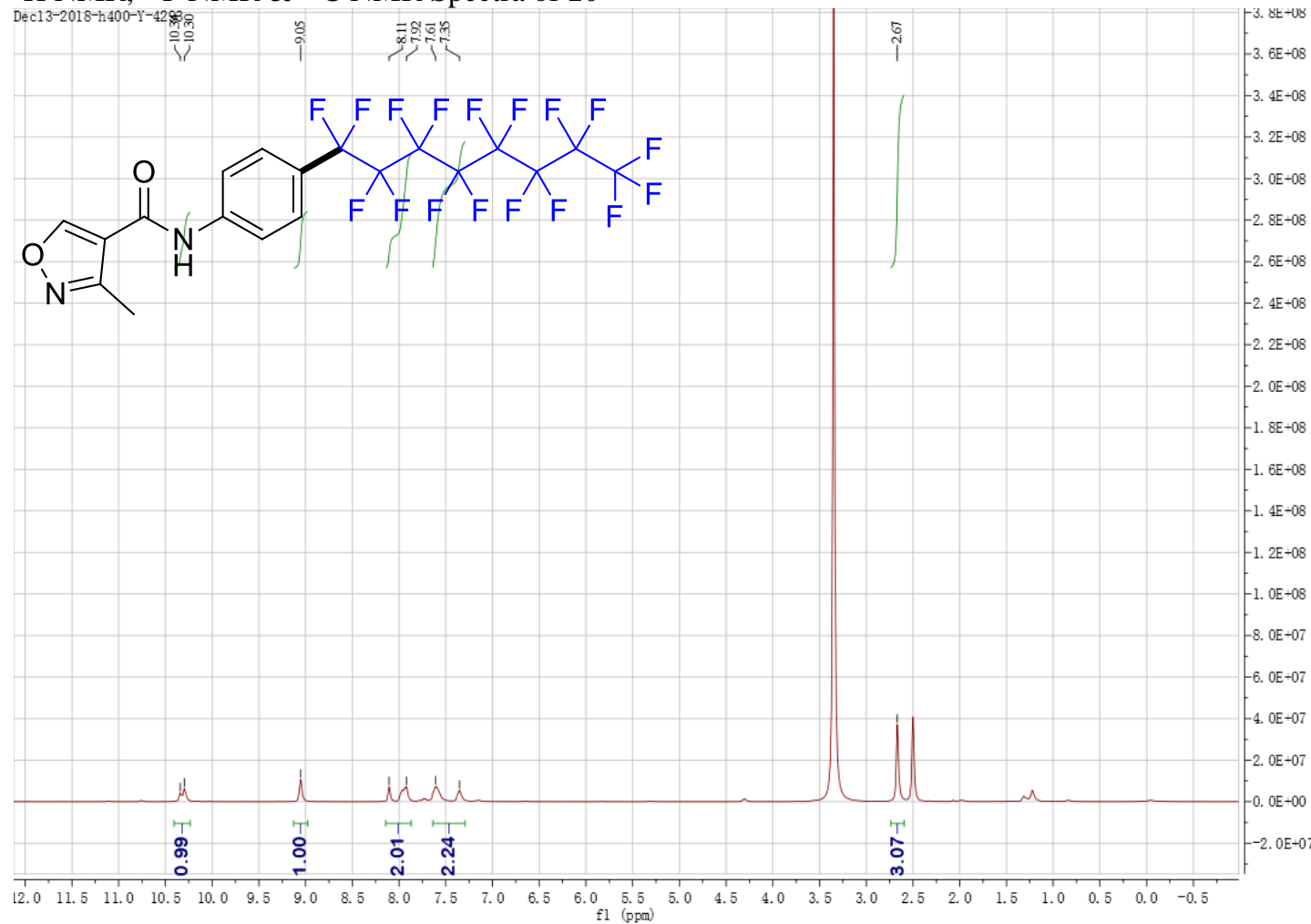


${ }^{19} \mathrm{~F}$ NMR Spectra of $\mathbf{1 0}$

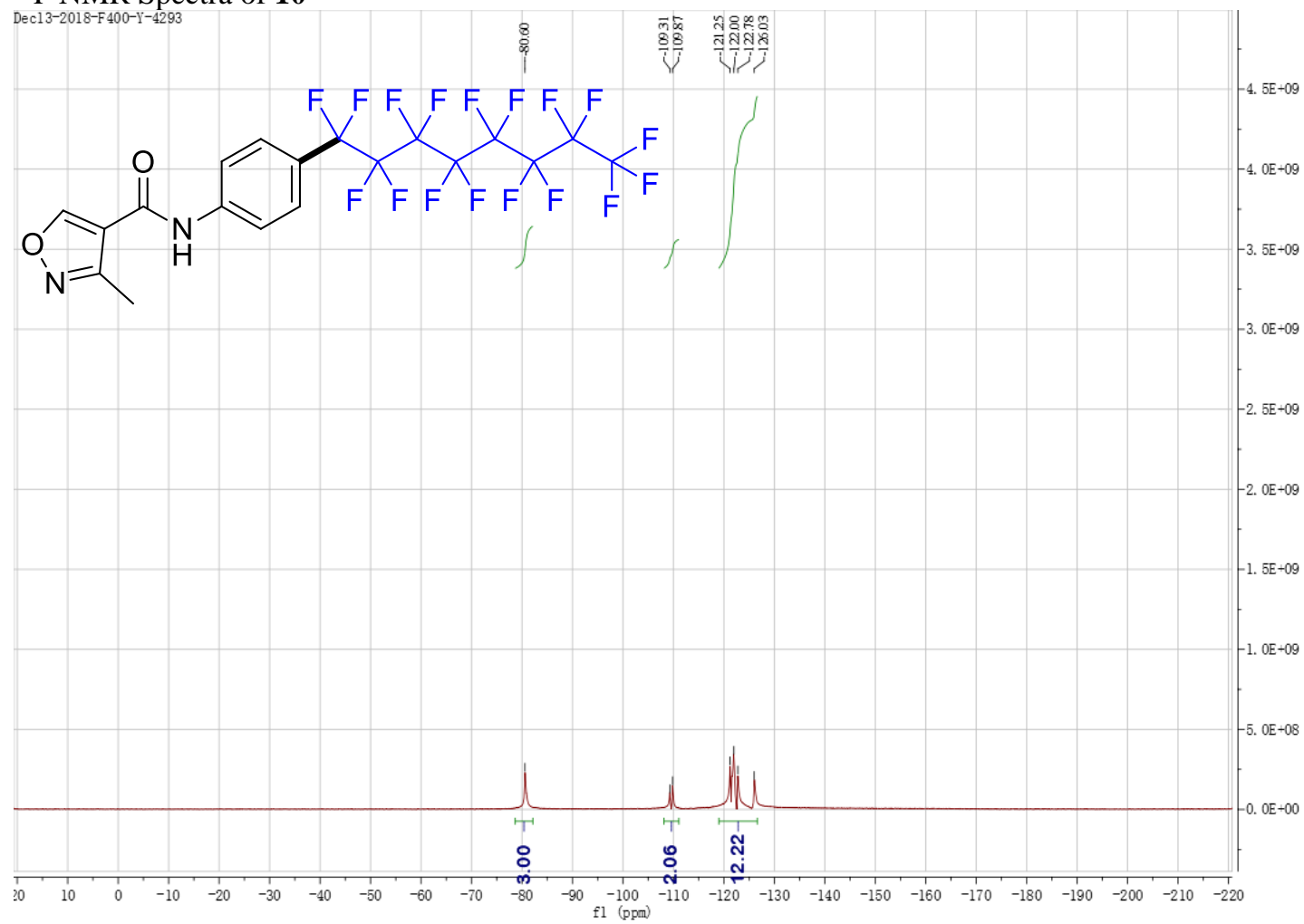

${ }^{13} \mathrm{C}$ NMR Spectra of $\mathbf{1 0}$

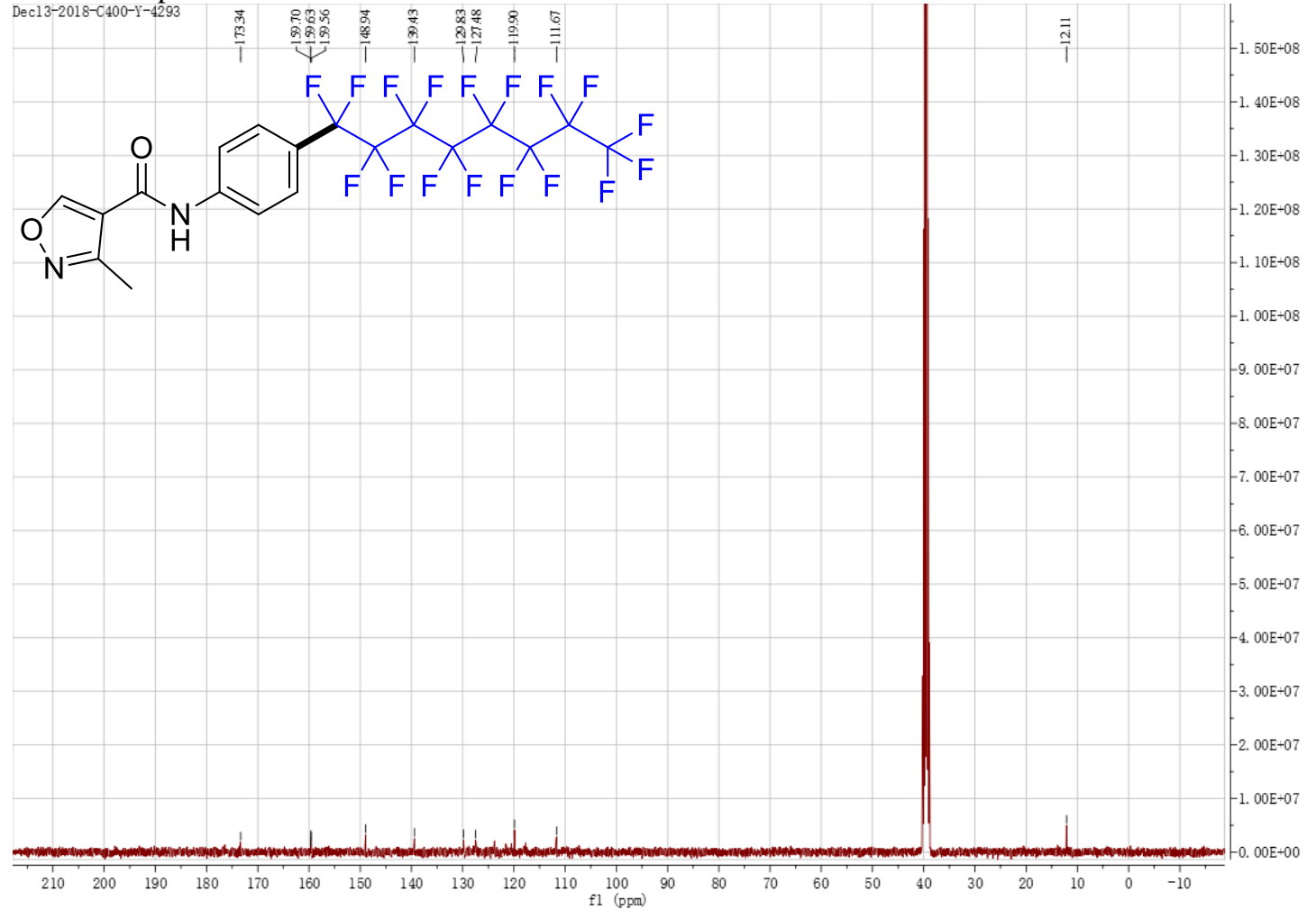


${ }^{1} \mathrm{H}$ NMR Spectra of $\mathbf{1 2}$

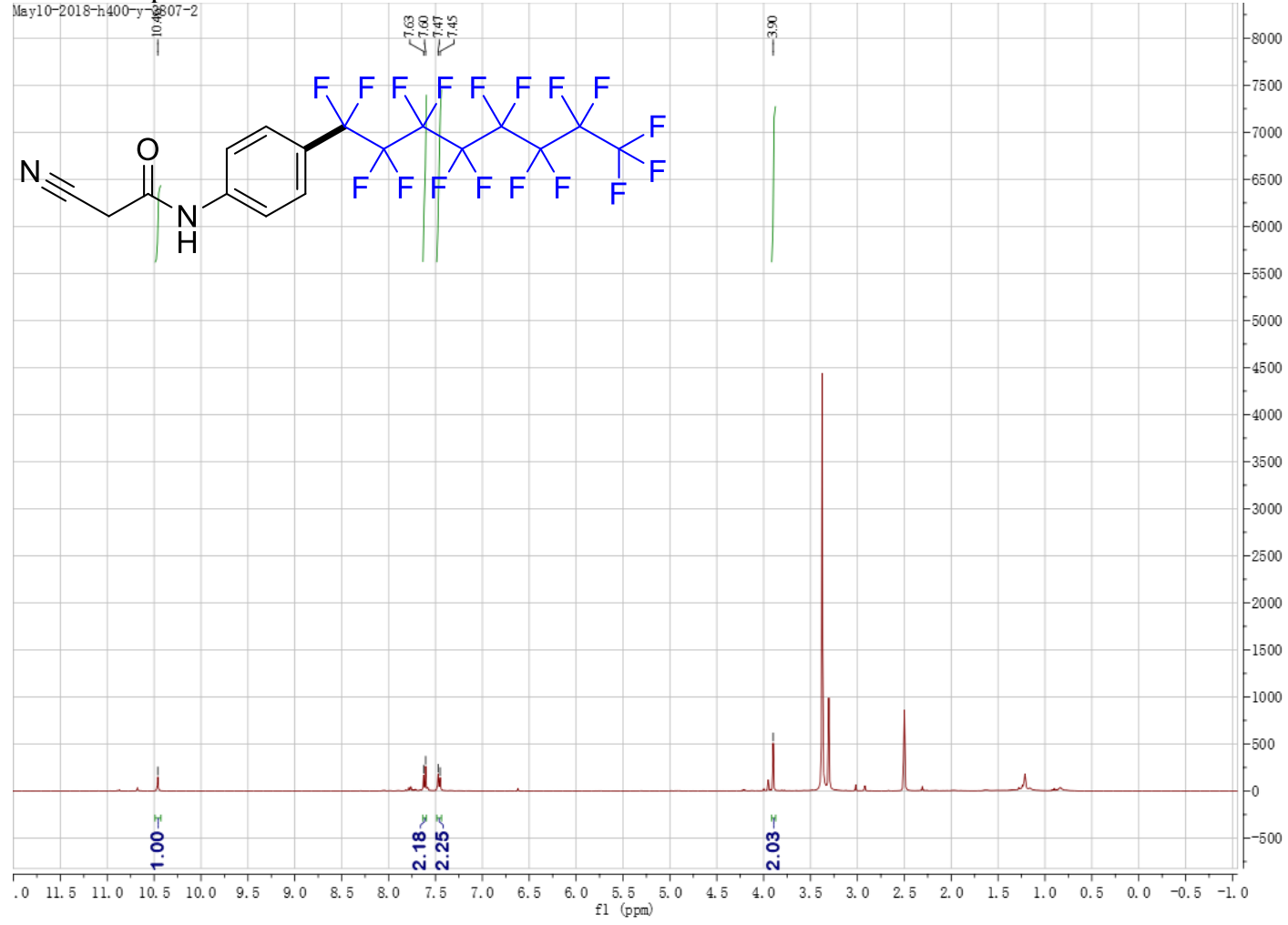

${ }^{13}$ C NMR Spectra of $\mathbf{1 2}$

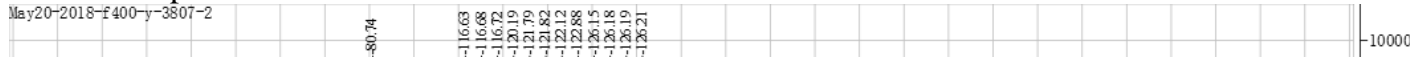
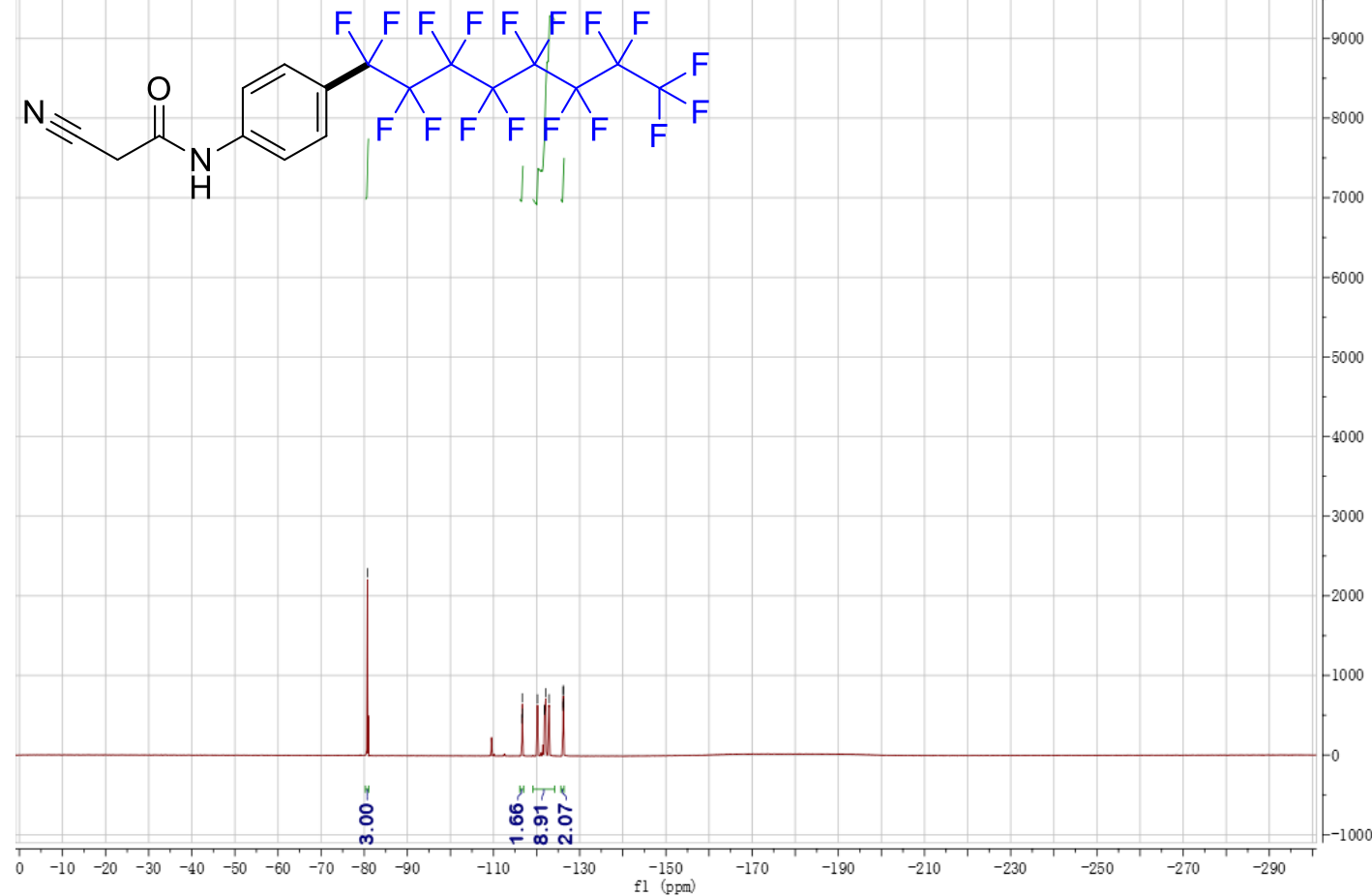
${ }^{13} \mathrm{C}$ NMR Spectra of $\mathbf{1 3}$

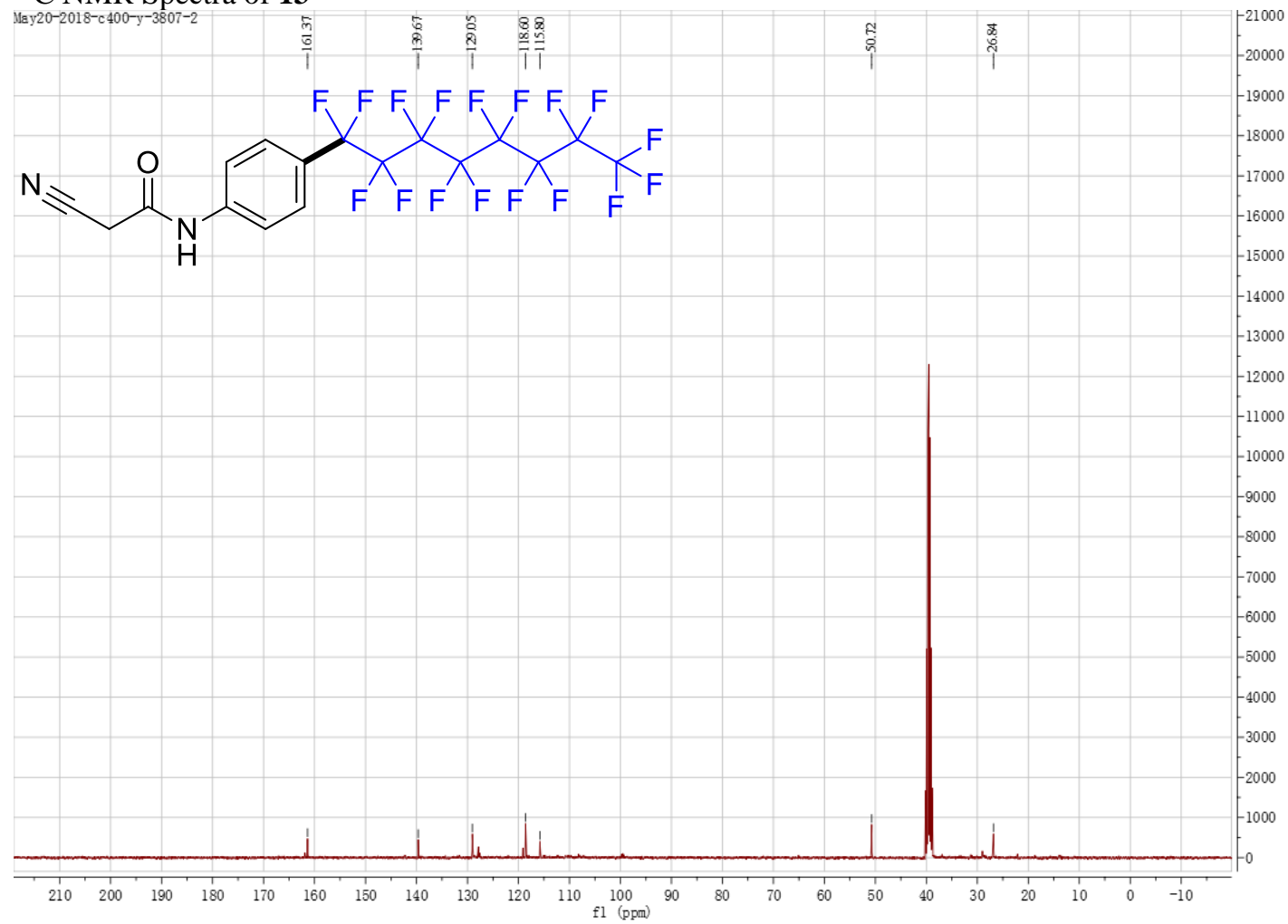

